\title{
Systematisches Transfermanagement für nachhaltige Führungskräftetrainings
}

\author{
Inaugural-Dissertation \\ in der Fakultät Humanwissenschaften \\ der Otto-Friedrich-Universität Bamberg
}

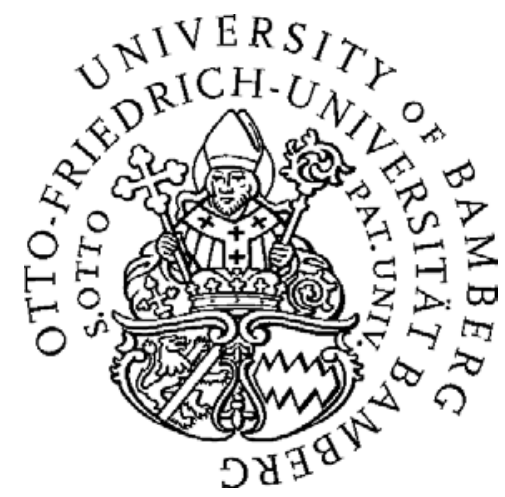

vorgelegt von

Belinda Maren Henrike Seeg

aus Mühlacker

Bamberg, den 31.03.2020 
Tag der Disputation: $\quad 26.06 .2020$

Dekan: $\quad$ Universitätsprofessor Dr. Jörg Wolstein

Betreuerin: Universitätsprofessorin Dr. Astrid Schütz

Weitere Gutachterin: Universitätsprofessorin Dr. Julia Franz

Dieses Werk ist als freie Onlineversion über das Forschungsinformationssystem (FIS; https://fis.uni-bamberg.de) der Universität Bamberg erreichbar. Das Werk steht unter der CCLizenz CC-BY. (c) $\underset{\mathrm{Br}}{\mathrm{Br}}$

URN: urn:nbn:de:bvb:473-irb-482565

DOI: https://doi.org/10.20378/irb-48256 



\section{Inhaltsverzeichnis}

Abbildungsverzeichnis ......................................................................... IV

Tabellenverzeichnis ................................................................................. V

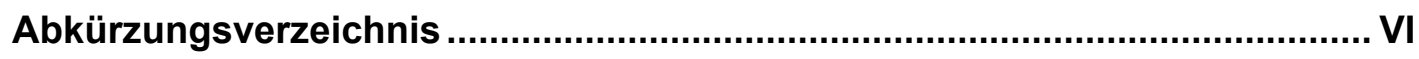

Strukturierte Zusammenfassung ........................................................... VII

1 Transfermanagement für nachhaltige Führungskräftetrainings ............1

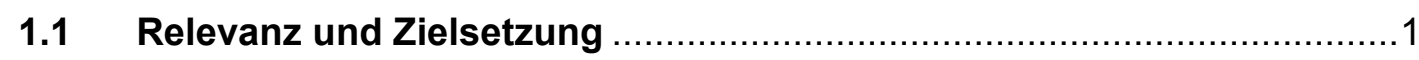

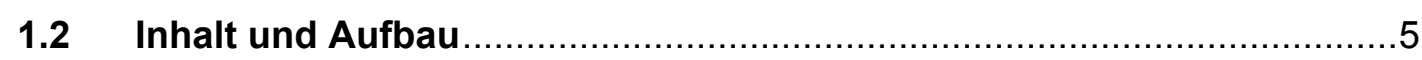

2 Zentrale Konzepte des Transfers von Führungskräftetrainings.............7

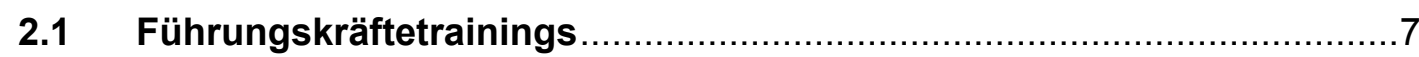

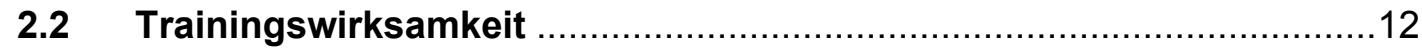

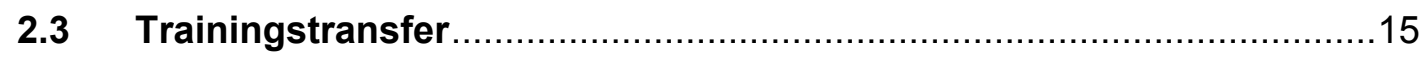

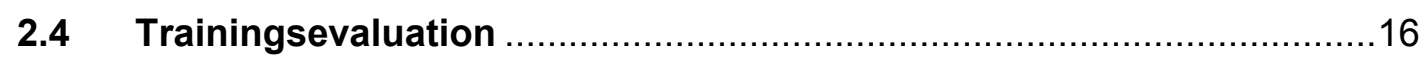

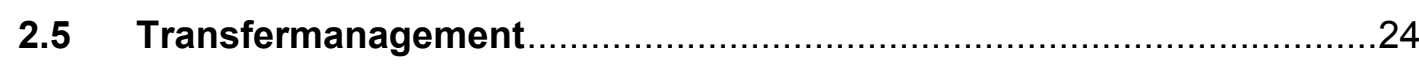

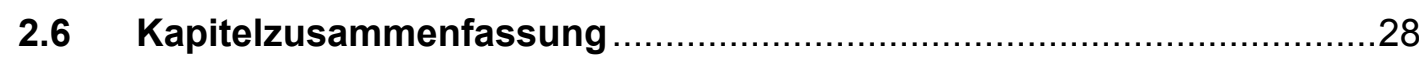

3 Zentrale Forschungsbefunde über Trainingswirksamkeit ...................30

3.1 Ergebnisorientierte Trainingsforschung ........................................ 31

3.1.1 Metaanalytisch bestätigte Befunde .......................................... 31

3.1.2 Eigenschaften von Evaluationsstudien ....................................40

3.1.3 Zusammenfassung ergebnisorientierter Trainingsforschung ..........41

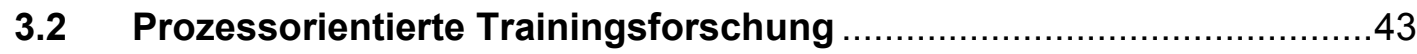

3.2.1 Befunde zu Determinanten des Trainingstransfers .......................45

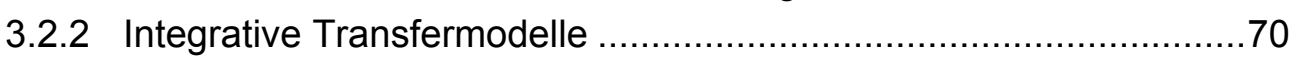

3.2.3 Zusammenfassung prozessorientierter Trainingsforschung ...........76

3.3 Research-Practice-Gap der Transferforschung ............................. 81

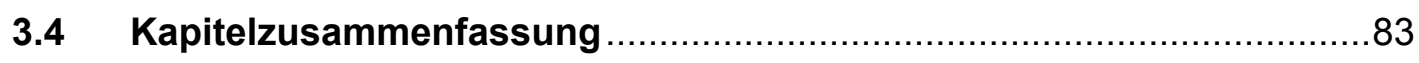

$4 \quad$ Forschungsgegenstand und konkrete Forschungsfragen ...................85

4.1 Ableitung von Hypothesen und Überprüfungsmethoden .....................85

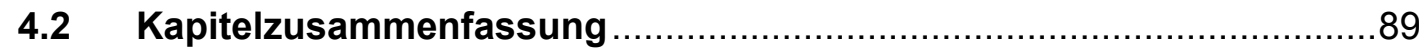

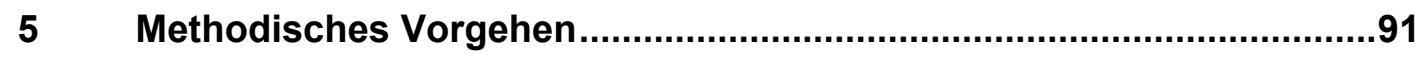

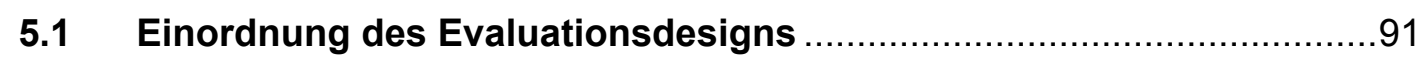

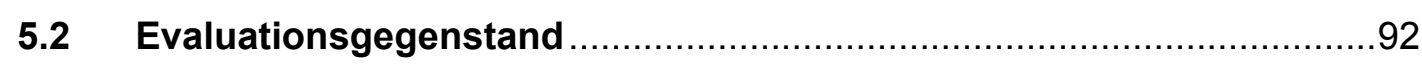

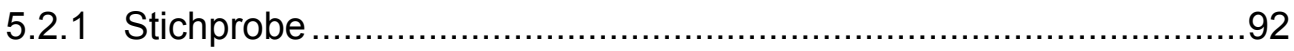

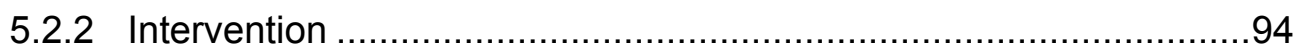




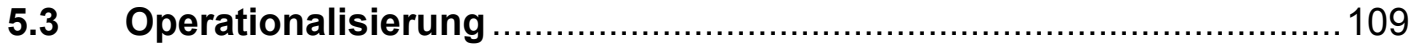

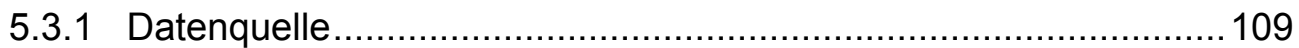

5.3.2 Rücklaufquote ............................................................. 110

5.3.3 Umgang mit fehlenden Werten ................................................. 110

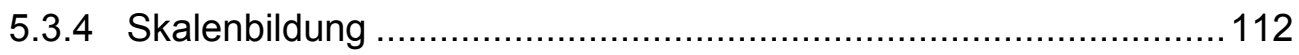

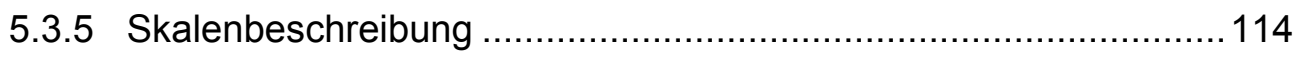

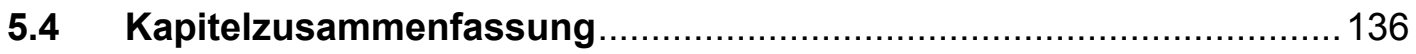

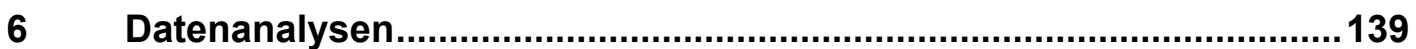

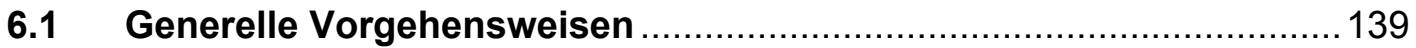

6.2 Ergebnisorientierte Wirksamkeitsüberprüfung …........................... 143

6.3 Prozessorientierte Wirksamkeitsüberprüfung ….......................... 148

6.3.1 Erklärung individueller Transferleistung .................................... 148

6.3.2 Erklärung primärer Transferdeterminanten............................... 158

6.4 Metaevaluation der Wirksamkeitsüberprüfungen ........................... 171

6.4.1 Evaluationsdesign........................................................ 171

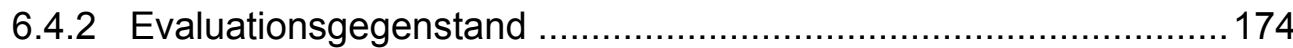

6.4.3 Operationalisierung ............................................................. 176

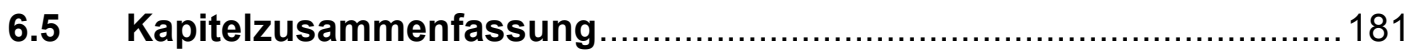

$7 \quad$ Leitfaden für systematisches Transfermanagement............................184

7.1 Transfermanagementmaßnahmen vor Trainings ............................. 185

7.2 Transfermanagementmaßnahmen während Trainings ….................. 187

7.3 Transfermanagementmaßnahmen nach Trainings........................... 189

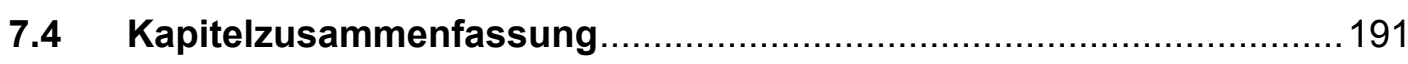

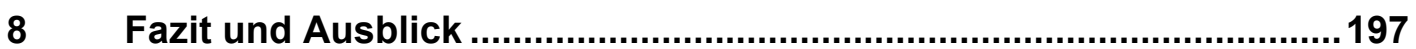

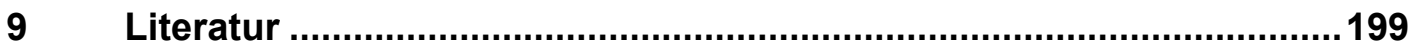




\section{Abbildungsverzeichnis}

Abbildung 1: Prozessmodell formeller Personalentwicklung nach Solga et al.

(2011, S. 24); eigene Darstellung.

Abbildung 2: Kompetenzklassen beruflicher Handlungskompetenz in Anlehnung an Roth (1971) und North et al. (2018); eigene Darstellung. ..........10

Abbildung 3: Ablauf des Behavior Modeling Trainings in Anlehnung an Taylor et al. (2005); eigene Übersetzung und Darstellung........................11

Abbildung 4: Vier Ebenen des Trainingserfolgs und mögliche Operationalisierungen nach Kauffeld (2016, S. 27); eigene Darstellung.

Abbildung 5: Kirkpatricks Modell der Trainingsevaluation zur Wirksamkeitsüberprüfung von Führungskräftetrainings nach Felfe und Franke (2014, S. 44); eigene Darstellung. ...........................14

Abbildung 6: CIPP-Evaluationsmodell von Stufflebeam $(1983,2004)$; eigene Übersetzung und Darstellung...................................................19

Abbildung 7: Prozess einer systematischen Trainingsevaluation nach Solga (2011a, S. 373); eigene Darstellung. ..........................................21

Abbildung 8: Transferprozess nach Noe und Schmitt (1986); eigene Übersetzung und Darstellung.....................................................71

Abbildung 9: Transfermodell nach Baldwin und Ford (1988, S. 65); eigene Übersetzung und Darstellung.......................................................72

Abbildung 10: Modell des deutschen Lerntransfer-System-Inventars nach Kauffeld et al. (2008); eigene Darstellung...................................74

Abbildung 11: Zusammenfassung zentraler Wirkmechanismen des Trainingstransfers; eigene Darstellung. ......................................77

Abbildung 12: Transfermodell des Forschungsprojektes. .................................87

Abbildung 13: Häufigkeitsverteilung der Hierarchieebenen. ...............................93

Abbildung 14: Überblick über Aufbau der Trainingsreihe zur Mitarbeitergesprächsführung; eigene Darstellung. .......................99

Abbildung 15: Checkliste für Maßnahmen vor Trainings in Anlehnung an Salas et al. (2012); eigene Übersetzung und Darstellung.......................96

Abbildung 16: Checkliste für Maßnahmen während Trainings in Anlehnung an Salas et al. (2012); eigene Übersetzung und Darstellung.............102

Abbildung 17: Anleitung zur gemeinsamen Reflexion persönlicher Entwicklungsziele.

Abbildung 18: Checkliste für Maßnahmen nach Trainings in Anlehnung an Salas et al. (2012); eigene Übersetzung und Darstellung.............106

Abbildung 19: Karte zur Definition SMARTer Entwicklungsschritte mit Nachverfolgung. 107

Abbildung 20: Benötigte Stichprobengrößen für Auswertungen mit multiplen Regressionsanalysen mit einer Teststärke von 8 nach Miles und Shevlin (2001); eigene Darstellung basierend auf Field (2018), S. 390.

Abbildung 21: Explorative Annahmen zur Erklärung primärer

Transferdeterminanten. 


\section{Tabellenverzeichnis}

Tabelle 1: Zusammenfassung der Metaanalysen zur Effektivität von Führungskräfteentwicklungsmaßnahmen

Tabelle 2: Positive, negative und moderierte Zusammenhänge zwischen Teilnehmendenmerkmalen und Trainings- und Transfererfolg

Tabelle 3: Positive, negative und moderierte Zusammenhänge zwischen Arbeitsumgebung und Trainings- und Transfererfolg

Tabelle 4: Positive, negative und moderierte Zusammenhänge zwischen Trainingsgestaltung und Trainings- und Transfererfolg....

Tabelle 5: Überblick der Rücklaufquoten..................................................... 110

Tabelle 6: Skalenüberblick der Transferdeterminanten ................................... 118

Tabelle 7: Skalenüberblick des Trainings- und Transfererfolges .................... 127

Tabelle 8: Eingruppen-(Prä-)Postvergleich für Trainings- und Transfererfolgskriterien.

Tabelle 9: Wartelistenkontrollgruppenvergleich für Trainings- und Transfererfolgskriterien

Tabelle 10: Variablenübersicht mit Deskriptiva, Pearsons Korrelationen und Cronbachs Alphas

Tabelle 11: Hierarchische Regressionsanalysen der Hypothesenprüfung zur Erklärung von Transfer

Tabelle 12: Hierarchische Regressionsanalysen zur explorativen Erklärung von Transfer.

Tabelle 13: Regressionsanalysen der Hypothesenprüfung zur Erklärung der primären Transferdeterminanten

Tabelle 14: Regressionsanalysen zur explorativen Erklärung der primären Transferdeterminanten

Tabelle 15: Gegenüberstellung der Analysen zu Erklärung von Transfermotivation nach dem Training ohne versus mit Kontrolle des Ausgangswertes

Tabelle 16: Explorative Analysen zu Erklärung von erwarteter Anwendungsmöglichkeit nach dem Training

Tabelle 17: Explorative Analysen zu Erklärung von Transfermotivation nach dem Training.....

Tabelle 18: Explorative Analysen zu Erklärung von Kompetenz nach dem Training

Tabelle 19: Explorative Analysen zu Erklärung von Transfermöglichkeit in Transferzeit. 


\section{Abkürzungsverzeichnis}

AV Abhängige Variable

k Anzahl berücksichtigter Studien

LMX Leader-Member-Exchange

LTSI Learning Transfer System Inventory

M Mittelwert (arithmetisches Mittel)

MAG Mitarbeitergespräch

MLQ Multifactor Leadership Questionnaire

N Anzahl der berücksichtigten Probanden bzw. Datensätze

GLTSI German Learning Transfer System Inventory

SD Standardabweichung

SREIS Self-Rated Emotional Intelligence Scale

SWE Selbstwirksamkeitserwartung

TB Trainingsbaustein 


\section{Strukturierte Zusammenfassung}

Ziel: Aufgrund der zunehmenden Relevanz nachhaltig wirksamer Führungskräfteentwicklung, zielte dieses Forschungsprojekt auf die Erklärung von Erfolg und Wirksamkeit wissenschaftlich fundierter Transförderung bei Trainings zur Vermittlung zentraler Führungskompetenzen ab. Dafür wurde ein ganzheitlicher Evaluationsansatz durch Integration von Prozess- und Ergebnisüberprüfung realisiert.

Methode: Evaluationsgegenstand war eine transferförderlich gestalteten Trainingsreihe zur Vermittlung bewährter Führungsstrategien (transformationale, transaktionale und interaktionale Führung) mit 66 Führungskräften eines repräsentativen deutschen Unternehmens. Dafür wurden im Rahmen des Forschungsprojektes eine Trainingsreihe sowie ihre Evaluation konzipiert und durchgeführt. Im Rahmen einer längsschnittlichen Feldstudie zur Evaluation des Trainings- und Transfererfolges wurden Transferdeterminanten und intraindividuelle Veränderungen vor, während und ca. 7 Monate nach Durchführung erfasst. Basierend auf einem Eingruppen-Prä-Postdesign, wurde eine ergebnisorientierte Wirksamkeitsüberprüfung mittels t-Tests durchgeführt, während die prozessorientierte Wirksamkeitsüberprüfung regressionsanalytisch erfolgte.

Ergebnisse: Sieben Monate nach der Trainingsreihe zeigten sich erzielte Verhaltensänderungen anhand direkter und indirekter Veränderungsmessungen. Dies wurde durch einen Wartelistenkontrollgruppenvergleich weitestgehend bestätigt. Dass diese Effekte auch positiv wahrgenommen wurden bestätigten sowohl Selbst-, als auch Mitarbeitendeneinschätzungen. Somit kann, neben der Praxistauglichkeit transferförderlicher Trainingsgestaltung, auch deren Effektivität als bestätigt gelten. Die identifizierten Transfermechanismen weisen darauf hin, dass Transfer durch eine direkte Funktion aus Kompetenz, Transfermotivation und -möglichkeit definiert werden kann. Für die übrigen bestätigten Transferdeterminanten wird ein vorwiegend indirekter Effekt angenommen, der durch die drei genannten direkten Faktoren vermittelt wird.

Erkenntnisgewinn und Limitationen: Die Forschungslücke prozessorientierter Transferforschung besteht insbesondere in der Überprüfung ganzheitlicher Modelle über den Trainings- und Transferprozess hinweg. Die vorliegende Arbeit schlägt dafür einen fundierten, ganzheitlichen und praxisorientierten Evaluationsansatz für die Trainingsforschung und -praxis vor, um die reichhaltigen Erkenntnisse der Trainingsforschung für die Lösung des über Jahrzehnte anhaltende Transferproblems nutzbar zu machen. Der Einsatz mehrerer Messzeitpunkte, um Kontrolle von Ausgangswerten und Erfassung der Wirkmechanismen über den Trainings- und Transferprozess hinweg zu realisieren, bewährten sich ebenso wie die Kontrolle von Trainings- und Organisationssetting. Diesbezüglich kann die vorliegende Evaluation als ganzheitlicher im Vergleich zu bisheriger Forschung gelten. Zudem wird durch theoriegeleitete Priorisierung anhand einer strikten Differenzierung zwischen primären und sekundären Transferdeterminanten ein Ansatz zur Komplexitätsreduktion für zukünftige Transferforschung vorgeschlagen. Allerdings bestehen, aufgrund des Evaluationsgegenstandes eines unternehmensspezifischen Trainings mit begrenzter Stichprobe und des realisierten Evaluationsdesigns, Limitationen insbesondere bezüglich statistischer Power, interner und externer Validität. Daher ist weitere Forschung zur Validierung der identifizierten Transfermechanismen erforderlich. Zudem sollten qualitative Analysen eingesetzt werden, um die Modellannahmen umfassend zu überprüfen und Alternativerklärungen auszuschließen. 



\section{Transfermanagement für nachhaltige Führungskräftetrainings}

„Die Forschung zum Transfererfolg ist ein weites Feld, das bisher noch nicht ausreichend erforscht ist. Wer nachhaltige Weiterbildung betreiben will, muss der Frage nachgehen, ob der Weiterbildungseffekt auch tatsächlich im Arbeitsalltag anhält." (Rosenstiel, 2012)

\subsection{Relevanz und Zielsetzung}

Wirksame Personalentwicklungsmaßnahmen gewinnen für die deutsche Wirtschaft stetig an Bedeutung (z. B. Kauffeld, 2016; Seyda \& Placke, 2017). Aufgrund der sich schnell wandelnden Arbeitswelt und der zunehmend geforderten Agilität von Unternehmen, ist eine kontinuierliche Weiterentwicklung berufsrelevanter Kompetenzen essenziell. Nur so kann individuelle Leistungsfähigkeit an stetig neue berufliche Herausforderungen angepasst werden, um wirtschaftlichen Erfolg langfristig zu sichern. „Von entscheidender Bedeutung ist [dabei] der Grad, in dem Weiterbildungen wirklich helfen, besser arbeiten zu können“ (Kauffeld, 2016, S. 4). Das Ausmaß in dem erworbene Handlungskompetenzen von Personalentwicklungsmaßnahmen (Lernfeld) erfolgreich auf den Arbeitskontext (Funktionsfeld) übertragen werden und zu einer bedeutsamen Verbesserung der Arbeitsleistung führt, wird als Trainingstransfer bezeichnet (Baldwin \& Ford, 1988). Entsprechend betrachtet die Forschung Trainingstransfer als entscheidendes Maß der Wirksamkeit von Personalentwicklungsmaßnahmen.

Bereits 1953 wurde von Fleishman das sogenannte „Transferproblem“, also die unzureichende Übertragung trainierter Kompetenzen auf den Arbeitskontext, als zentrales Thema in die Trainingsforschung eingeführt. Seitdem hat die Forschung intensiv an der Ergründung und Lösung des Transferproblems gearbeitet, wodurch sich die Transferforschung über Jahrzehnte hinweg als Schlüsselthema der Personalentwicklung etabliert hat (Jeung, Yoon, Park \& Jo, 2011). Obwohl über die letzten Jahrzehnte ein beachtliches Volumen an Befunden und Handlungsempfehlungen zur Lösung des Transferproblems veröffentlicht wurde, hinterfragen Praxis und Forschung anhaltend die Wirksamkeit von Personalentwicklungsmaßnahmen. Dabei wird insbesondere der Wirkungsgrad formaler „off-the-job“1 Führungskräftetrainings zur Vermittlung von Softskills in Frage gestellt

${ }^{1}$ Gemäß der Kategorisierung von Personalentwicklungsmaßnahmen nach (Conradi, 1983) können drei Maßnahmenkategorien nach Lernort i. S. physischer und vor allem inhaltlicher Entfernung vom Arbeitsalltag unterschieden werden: „off-the-job“ (z. B. Training und Coaching), „near-the-job“ (z. B. Mentoring) und „on-the-job“ (z. B. individuelle Förderung mittels Alltagsfeedback, Aufgabenzuteilung, etc.). Zu beachten ist, dass die Kategoriegrenzen fließend sind 
(Kaiser \& Curphy, 2013; Laker \& Powell, 2011; Poell, 2017; J. Schwartz, Bersin \& Pelster, 2014; Staudt \& Kriegesmann, 1999) - obwohl (oder gerade weil) es das meist institutionalisierte Format der Personalentwicklung ist (Felfe, 2016; Felfe \& Franke, 2014; Lacerenza, Reyes \& Marlow, 2017; Martin, Epitropaki \& O’Broin, 2017; Seyda \& Placke, 2017). Manche Autoren ${ }^{2}$ kommen dabei zu dem Schluss, dass das Transferproblem mit einer Ablösung des klassischen Trainingsformats durch informelle „on-thejob“ Maßnahmen gelöst werden könnte (z. B. Poell, 2017; Staudt \& Kriegesmann, 1999). Andere Autoren zeigen dagegen das bedeutende Potential formeller Führungskräftetrainings auf (Felfe \& Franke, 2014; Lacerenza et al., 2017; Martin et al., 2017; Sørensen, 2017): "Leadership training is the most direct way to develop manager's leadership competencies, can cover many topics related to leadership, and typically occurs in small group classroom-type settings. Through various techniques (e.g., multisource feedback, reflection, role-playing) managers develop various leadership skills and competencies. The aim of the training is to change the way the leader behaves toward their followers, which can lead to improved follower well-being (such as enhanced job satisfaction and organizational commitment, or lower stress), team well-being (such as cohesiveness), and work performance" (Martin et al., 2017, S. 75). Diese Trainingseigenschaften und -effekte können nicht ohne Weiteres durch informellere „on-the-job“ Maßnahmen ersetzt werden, sondern stellen für nachhaltige Personalentwicklung eine wichtige komplementäre Ergänzung dar (Enos, Kehrhahn \& Bell, 2003; Kauffeld, 2016; Sparr, Knipfer \& Willems, 2017). So erscheint die Lösung des Transferproblems insbesondere für Führungskräftetrainings lohnend, um ihr mögliches Potential nutzbar zu machen und ihren Wirkungsgrad zu erhöhen, anstatt dieses zentrale Format der Personalentwicklung zu ersetzen.

Die Transferforschung liefert dafür bereits zahlreiche Befunde, Handlungsempfehlungen und Werkzeuge (z. B. Checklisten für transferförderliche Trainingsgestaltung oder Erhebungsinstrumente für Transferfaktoren und -kriterien, siehe Baldwin, Ford \& Blume, 2017; Bates, Holton \& Hatala, 2012; Ford, Baldwin \& Prasad, 2018; Grohmann \& Kauffeld, 2013; H. M. Hutchins, Burke \& Berthelsen, 2010; Martin et al., 2017; Salas, Tannenbaum, Kraiger \& Smith-Jentsch, 2012). Allerdings scheint die Trainingspraxis davon weitestgehend unberührt zu bleiben und setzt die Empfehlungen für fundierte Transferförderung kaum um (Huint \& Saks, 2003; Kaiser \& Curphy, 2013; Salas \& Kosarzycki,

und auch einige Maßnahmen als Mischformen konzipiert werden, um die jeweiligen Vorteile zu kombinieren bzw. Nachteile zu kompensieren.

2 In der vorliegenden Arbeit wurde eine möglichst genderneutrale Sprachform gewählt. Aus Gründen der besseren Lesbarkeit wurde dennoch in gängigen Einzelfällen die Sprachform des generischen Maskulinums verwendet, die jedoch auch geschlechtsunabhängig verstanden werden soll. 
2003; Wang \& Wilcox, 2014; Weinbauer-Heidel, 2016). Es finden in der Trainingspraxis zudem kaum systematische Analysen des tatsächlichen Transferwirkungsgrades und der dahinterliegenden Transfermechanismen statt (Wang \& Wilcox, 2014). Mit dem von Eichenberger (1990) formulierten Titel „Millionen für Bildung, Pfennige für Evaluation“ kann auch die aktuelle Situation treffend beschrieben werden. Obwohl Bildungsverantwortliche aus der Wirtschaft das Transferproblem als zentrale Herausforderung der Personalentwicklung betrachten und geringe Wirkungsgrade von Trainings lamentieren, wird die "Black Box“ des Trainingstransfers in der Praxis selten überprüft und kaum gezielt optimiert (Diesner \& Seufert, 2010, 2013; Fandel-Meyer, Schneider, Seufert, Meier \& Schuchmann, 2015). Neben der Lösung des Transferproblems ist daher auch der Wissenschaftstransfer der Transferforschung ein zentrales Handlungsfeld.

Entsprechend besteht das Transferproblem nachhaltiger Personalentwicklung in einem doppelten Sinn - einerseits bezogen auf die Übertragung von Trainingsinhalten auf den Arbeitskontext, andererseits bezogen auf die Übertragung vorhandener Forschungserkenntnissen zur Lösung des Transferproblems auf die Trainingspraxis. Forschende haben schon mehrfach versucht zu ergründen, warum die Erkenntnisse aus einem Jahrhundert der Trainingsforschung über Trainingseffektivität und -transfer von der Personalentwicklungspraxis kaum genutzt werden, während der Bedarf schon längst erkannt wurde (Ford et al., 2018; Salas \& Kosarzycki, 2003; Tonhäuser \& Bücker, 2016; Weinbauer-Heidel, 2016). Angenommene Gründe sind insbesondere mangelnde Praxisorientierung der Forschung und unzureichende Ressourcen (Zeit, Geld und Knowhow) für ein systematisches Transfermanagement in der Trainingspraxis (Hinrichs, 2016; H. M. Hutchins et al., 2010; Salas et al., 2012; Solga, 2011b; Weinbauer-Heidel, 2016). Eine mögliche Ursache dafür kann, einer aktuellen Zusammenfassung der Transferforschung zu Folge, in einem sogenannten „Bricklayer-Phänomen“ gesehen werden (Baldwin et al., 2017): Die Transferforschung hat über Jahrzehnte die Ergründung einzelner Zusammenhängen intensiviert, weitere potentielle Transferfaktoren identifiziert und bestehende Transfermodelle stetig erweitert. Auch wenn sich daraus viele wichtige Erkenntnisse für die Transferförderung ergeben, hat es zur Konsequenz, dass integrative Transfermodelle sowohl für ganzheitliche Überprüfungen als auch für die Trainingspraxis sehr komplex sind und dennoch die Realität nicht vollständig abbilden können. So hat sich die Transferforschung in ihrer eigenen Komplexität verfangen. Es fehlt insbesondere eine fundierte Priorisierung für den gezielten Ressourceneinsatz für Transfermanagementmaßnahmen. Um die reichhaltigen Erkenntnisse der Transferforschung tatsächlich für die Praxis nutzbar zu machen, rufen die Autoren daher zu einer praktikablen Konsolidierung der Einzelbefunde auf: "There is potential for a much greater yield on the enormous organizational investments in training - but only if researchers are conducting 
the types of studies and synthesizing the knowledge learned in ways that stimulate thinking about transfer and ultimately are meaningful to professionals" (Ford et al., 2018, S. 202).

Daran anknüpfend adressiert die vorliegende Arbeit das Jahrzehnte beklagte Transferproblem. Einerseits wird dafür eine ganzheitliche Betrachtung der Trainingswirksamkeit und ihrer Wirkmechanismen vorgenommen. Andererseits reduziert eine Fokussierung auf primäre Transferfaktoren die Komplexität des Modells zur Evaluation und Förderung von Transfer. Durch das vorliegende Forschungsprojekt soll überprüft werden, inwiefern die Erkenntnisse zur Transferförderung praxistauglich und effektiv umgesetzt werden können. Da formelle Führungskräftetrainings zur Vermittlung interaktionaler Kompetenzen das bedeutendste Personalentwicklungsformat darstellen, ist dieses Format Forschungsgegenstand der vorliegenden Arbeit, die drei übergeordnete Ziele verfolgt:

- Überprüfung der Praxistauglichkeit und Wirksamkeit der Erkenntnisse zur Transferförderung aus der Trainingsforschung im Kontext von Führungskräftetrainings deutscher Unternehmen

- Ergründung primärer Transfermechanismen und ihrer zielgerichteten Fördermöglichkeiten

- Ableitung eines wissenschaftlich fundierten Leitfadens für ein systematisches Transfermanagement: Zielgerichtete Maßnahmenempfehlungen zur Überprüfung und Förderung von Aufbau und Anwendung beruflicher Handlungskompetenzen, die durch Führungskräftetrainings vermittelt werden.

Das dafür durchgeführte Forschungsprojekt umfasst einerseits eine wissenschaftlich fundierte Konzeption und Durchführung von Trainingseinheiten für nachhaltig wirksame Führungskräfteentwicklung. Andererseits ist die Konzeption und Durchführung einer maßgeschneiderten, multimodalen ${ }^{3}$ und längsschnittlichen ergebnis- und prozessbezogene Evaluation zur Überprüfung von Transfererfolg und -mechanismen Gegenstand der vorliegenden Arbeit. So verknüpft diese Arbeit diagnostische und interventionspsychologische Konzepte, hypothesenprüfende und theoriebildende Methoden sowie Wissenschafts- und Anwendungsbezug. Dadurch können nicht nur Praktikabilität und Wirksamkeit bisheriger Handlungsimplikationen überprüft, sondern auch dahinterliegenden Transfermechanismen ergründet werden. Auf dieser Basis können schließlich fundierte, praktikable und wirksame Lösungen für das seit Jahrzehnten ungelöste und zunehmend bedeutendere Transferproblem für Wissenschaft und Praxis gegeben werden.

${ }^{3}$ durch Evaluationskriterien auf vier Wirksamkeitsebenen nach Kirkpatrick (1967, 1998; siehe Abschnitt 2.2 ab Seite 11). 


\subsection{Inhalt und Aufbau}

Nachdem einleitend Relevanz und übergeordnete Zielsetzung des Forschungsprojekts vorgestellt wurden, folgt nun eine Zusammenfassung von Aufbau und Inhalt der vorliegenden Forschungsarbeit.

Für eine theoretische Einbettung zentraler Konzepte wird zunächst das hier zugrunde gelegte Verständnis in Kapitel 2 (ab Seite 7) definiert. Dabei wird das zugrunde gelegte Verständnis von Führungskräftetrainings, Trainingswirksamkeit und -transfer sowie deren Evaluation und systematisches Management durch inhaltliche und begriffliche $\mathrm{Ab}$ grenzungen präzisiert, um die Einordnung der Forschungserkenntnisse zu unterstützen.

Der darauf basierende Literaturüberblick des Forschungsstands zur Wirksamkeit von Führungskräftetrainings ist in Kapitel 3 (ab Seite 30) gesammelt und integriert. Nach einer Zusammenfassung der ergebnisorientierten Evaluationsforschung und ihrer Erkenntnisse zur Wirksamkeit formeller Führungskräfteentwicklung, folgt ein Überblick der prozessorientierten Evaluationsforschung zu Trainingstransfer und dessen Wirkmechanismen. Zudem wird ein Überblick über den Research-Practice-Gap bezüglich fundierter Transfermanagementaktivitäten in der Trainingspraxis gegeben.

Von dem aufgezeigten Forschungsstand und den bestehenden Forschungslücken der Transferforschung werden Forschungsgegenstand und Hypothesen der vorliegenden Arbeit in Kapitel 4 (ab Seite 85) abgeleitet. Um zu ergründen, warum das Transferproblem trotzt zahlreicher Forschungsarbeiten zu dessen Lösung durch fundierte Empfehlungen für transferförderliche Trainingsgestaltung bis heute ungelöst ist, sollen in einem Anwendungsversuch die Praxistauglichkeit, Wirksamkeit und Wirkmechanismen zentraler Handlungsimplikationen zur Transferförderung überprüft werden.

Kapitel 5 (ab Seite 91) stellt das methodische Vorgehen zur Beantwortung der Forschungsfragen wird im daran anschließenden vor. Nach einer übergeordneten Einordung des Forschungsprojektes anhand zentraler Eigenschaftsdimensionen der Evaluationsforschung, wird der konkrete Studienaufbau anhand von Stichprobe, Trainingsreihe bzw. Intervention und Evaluationsdesign beschrieben. Zudem wird die Operationalisierung der Evaluationsvariablen erläutert.

In Kapitel 6 (ab Seite 139) sind die Ergebnisse der Hypothesenprüfungen zusammengefasst. Dabei werden zunächst die ergebnisorientierten Analysen zur multidimensionalen Überprüfung der Wirksamkeit der transferförderlich gestalteten Trainingsreihe beschrieben und diskutiert. In einem zweiten Schritt werden daran anknüpfend die prozessorientierten Analysen zur Überprüfung der dahinterliegenden Wirkmechanismen dargestellt. Für eine tiefergehende Ergründung insbesondere der zeitlichen Folge und 
der relativen Relevanz der einzelnen Transferfaktoren für zielgerichtete Transfermanagementempfehlungen, werden weitere explorativen Auswertungen vorgestellt. So können die Annahmen aus den Hypothesentestungen gegengeprüft und durch weiterführende Anhaltspunkte zu Wirkmechanismen des Trainingstransferprozesses ergänzt werden. Durch eine übergeordnete Metaevaluation des Evaluationsprozesses und dessen Ergebnisse werden Limitationen kritisch diskutiert und offen gebliebene Fragen aufgezeigt.

Vor diesem Hintergrund werden Erkenntnisgewinn und theoriebildende Konsequenzen der verschiedenen Analyseebenen in Kapitel 7 (ab Seite 184) zusammenfassend integriert. Daraus resultierende Handlungsimplikationen für die Praxis sind dafür in einem Leitfaden für systematisches Transfermanagement zusammengefasst.

In Kapitel 8 (ab Seite 197) erfolgt eine kritische Reflexion des Forschungsprojekts. Das abschließende Fazit mit Ausblick fasst den generierten Erkenntnisgewinn und offene Fragen zusammen.

Für einen schnellen Überblick des umfassenden Forschungsprojektes und seiner Ergebnisse sind jeweils am Kapitelende die zentralen Inhalte zusammengefasst und grau hinterlegt. 


\section{Zentrale Konzepte des Transfers von Führungskräftetrainings}

Bevor ein Literaturüberblick den Forschungsstand zur Wirksamkeit von Führungskräftetrainings und zur Evaluationsforschung des Transferprozesses zusammenfasst, werden in diesem Kapitel zentrale Konzepte dieses Forschungsgegenstands präzisiert. Damit soll eine Einordnung der Forschungsbefunde und abgeleiteten Forschungsfragen unterstützt werden.

\subsection{Führungskräftetrainings}

Das diesem Forschungsprojekt zugrundeliegende Verständnis von Führungskräftetrainings folgt der Definition von Felfe und Franke (2014) und umfasst „Maßnahmen der Personalentwicklung zur systematischen Erweiterung und Förderung der für die Führung bzw. Mitarbeiterführung erforderlichen Handlungskompetenz. Im Mittelpunkt stehen [sic] das Training von konkretem Verhalten und die verhaltenssteuernden Überzeugungen, Einstellungen und Werte" (S. 6).

Für die vorliegende Arbeit wird entsprechend unter dem Führungsbegriff Mitarbeiterführung bzw. personale Führung verstanden. „Personale Führung lässt sich als unmittelbare, absichtliche und zielbezogene Einflussnahme von bestimmten Personen (Vorgesetzte) auf andere (Untergebene) mit Hilfe der Kommunikationsmittel bestimmen“ (Rosenstiel, 2006, S. 355). Damit wird nur ein Teilbereich der Führung in Organisationen betrachtet. „Unternehmensführung“, die sich mit der Planung, Steuerung und Kontrolle einer Organisation beschäftigt (Becker, 2013), wird hier ausgeklammert. Stattdessen stehen die dyadischen Interaktionsprozesse zwischen Vorgesetzten und Mitarbeitenden im Fokus.

In der Personalentwicklungsliteratur wird der Trainingsbegriff häufig als Sammelbegriff für Personalentwicklungsmaßnahmen verwendet (insbesondere in der englischsprachigen Literatur) und umfasst sämtliche Maßnahmen zur systematischen Förderung beruflicher Handlungskompetenz - von zentral organisierten Maßnahmen auf Unternehmensebene bis hin zu selbstgesteuerten Initiativen auf individueller Ebene (Goldstein \& Ford, 2002). Darin ist auch informelle Personalentwicklung eingeschlossen. Als Basisverständnis des Teilbereichs formeller Personalentwicklung wird hier folgende Definition und Umsetzungsempfehlung herangezogen: „Förderung beruflich relevanter Kenntnisse, Fertigkeiten, Einstellungen etc. durch Maßnahmen der Weiterbildung, der Beratung, des systematischen Feedbacks und der Arbeitsgestaltung. Dabei sollten die Ziele und Inhalte von Personalentwicklung unternehmensstrategisch begründet sein, d.h. auf 
Kompetenzen fokussieren, die zur Verwirklichung strategischer Unternehmensziele benötigt werden“ (Solga, Ryschka \& Mattenklott, 2011, S. 19). Diese Definition wird von den Autoren durch ein Prozessmodell der Personalentwicklung veranschaulicht, das in Abbildung 1 die konkreten Aufgabenpakete zur Umsetzung von nachhaltig wirksamen Personalentwicklungsmaßnahmen systematisch zusammenfasst. Demnach sollte jede Maßnahme mit einer aus den strategischen Unternehmenszielen abgeleiteten Bedarfsanalyse starten, die als Basis der Konzeption und Durchführung dient. So soll sichergestellt werden, dass die Transferleistung inhaltlich zum Kontext passt und die übergeordneten Unternehmensziele unterstützt. Prozessbegleitend muss zudem durch Transfermanagement im Lernfeld und Arbeitsalltag die Anwendung des Gelernten unterstützt werden und schließlich durch Evaluation aller Schritte die Qualität nicht nur überprüft, sondern auch gesichert werden.

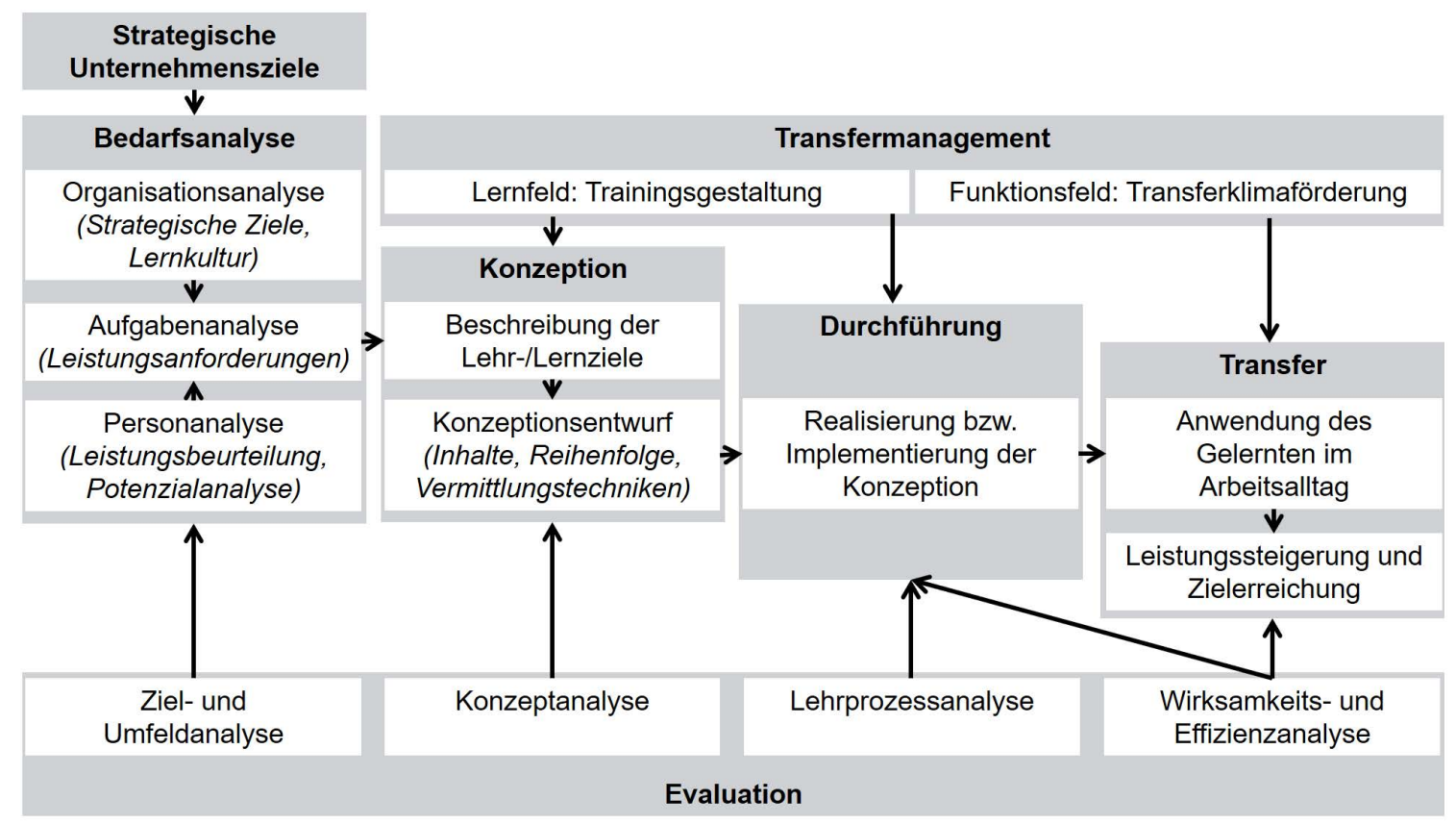

Abbildung 1: Prozessmodell formeller Personalentwicklung nach Solga et al. (2011, S. 24); eigene Darstellung.

In der vorliegenden Arbeit wird der Trainingsbegriff im engeren Sinne verwendet und als eine Maßnahmenart formeller Personalentwicklung verstanden. Die in Abbildung 1 dargestellten Umsetzungsempfehlungen sind insbesondere für formale Trainings gültig. Bei der Interpretation und Nutzung von Forschungsbefunden muss jedoch auf das jeweilige Begriffsverständnis geachtet werden. Insbesondere für Transfererkenntnisse kann es einen Unterschied darstellen, ob sich die Inhalte konkret auf Trainings im engeren Sinne beziehen oder auf zusammengefasste Betrachtungen sämtlicher Maßnahmen formeller Personalentwicklung einschließlich „on-the-job“ Verfahren. Denn die Übertragung von Trainingsinhalte auf den Arbeitsalltag folgt der Faustformel: Je „näher“ der Lernort am 
Arbeitsort ist, desto geringer ist das Transferproblem, da die Übertragung neu erlernter Kompetenzen oder Wissensaspekte durch die Übereinstimmung von Lern- und Anwendungsort erleichtert bzw. gar nicht erst erforderlich ist (Kauffeld, 2016). Umgekehrt bedeutet dies für Trainings als „off-the-job“ Maßnahme, dass der Transfer eine zentrale Herausforderung darstellt und insbesondere bei dieser Form der Personalentwicklung systematisch gefördert werden muss. Dabei spielen Führungskräftetrainings eine zentrale Rolle (Felfe \& Franke, 2014; Kanning, 2014; Lacerenza et al., 2017; Martin et al., 2017). Einerseits, weil Führungskräftetrainings den größte Kostenblock und die am meisten institutionalisierte Form der Personalentwicklung darstellen (Ho, 2016; Lacerenza et al., 2017; O'Leonard, 2014; Seyda \& Placke, 2017; Sørensen, 2017); andererseits, weil sich Führungskompetenzen nicht nur auf die Führungskräfte selbst, sondern auch maßgeblich auf Leistung, Motivation und Wohlbefinden ihrer Mitarbeitenden auswirken (Diebig, Bormann \& Rowold, 2016; Dulebohn, Bommer, Liden, Brouer \& Ferris, 2012; Felfe, 2016; Felfe \& Franke, 2014). Denn Führungskräfte wirken als Multiplikatoren: Sie besitzen neben ihrer Weisungsfunktion auch eine starke Vorbildwirkung für unterstellte Mitarbeitende. Das hat weitreichende Konsequenzen für die Zufriedenheit, Gesundheit und Leistung der Mitarbeitenden und damit auch für den wirtschaftlichen Erfolg eines Unternehmens (Day, Zaccaro \& Halpin, 2005; Felfe, 2016; Kozlowski, Watola, Jensen, Kim \& Botero, 2009; Morgeson, DeRue \& Karam, 2010; Volmer, 2015). In der vorliegenden Arbeit geht es daher konkret um die Optimierung formeller „off-thejob“ Weiterbildungsmaßnahmen für Führungskräfte, die in Abstand zum Alltag stattfinden, um Gruppen in geschütztem Rahmen anzuleiten Erfahrungen auszutauschen, Erkenntnisse zu gewinnen und neue Verhaltensweisen auszuprobieren. Denn diese Form der Führungskräfteentwicklung,$\ldots$ is the most direct way to develop manager's leadership competencies, can cover many topics related to leadership, and typically occurs in small group classroom-type settings. Through various techniques (e.g., multisource feedback, reflection, role-playing) managers develop various leadership skills and competencies" (Martin et al., 2017, S. 75). Dabei vermittelt klassischerweise eine Trainerin oder ein Trainer zwischen acht und vierzehn Teilnehmenden Wissen, Erkenntnis und/oder Zielverhaltensweisen beruflicher Handlungskompetenz (Kanning, 2014).

In Anlehnung an das Basismodell beruflicher Handlungskompetenz nach Roth (1971) setzt sich diese aus vier Kompetenzklassen zusammen, die in Fach-, Methoden-, Sozialund Selbstkompetenzen unterschieden werden (Hülshoff et al., 2010; Kauffeld, 2006; North, Reinhardt \& Sieber-Suter, 2018). Die jeweiligen Aspekte der einzelnen Kompetenzklassen sind in Abbildung 2 dargestellt. 


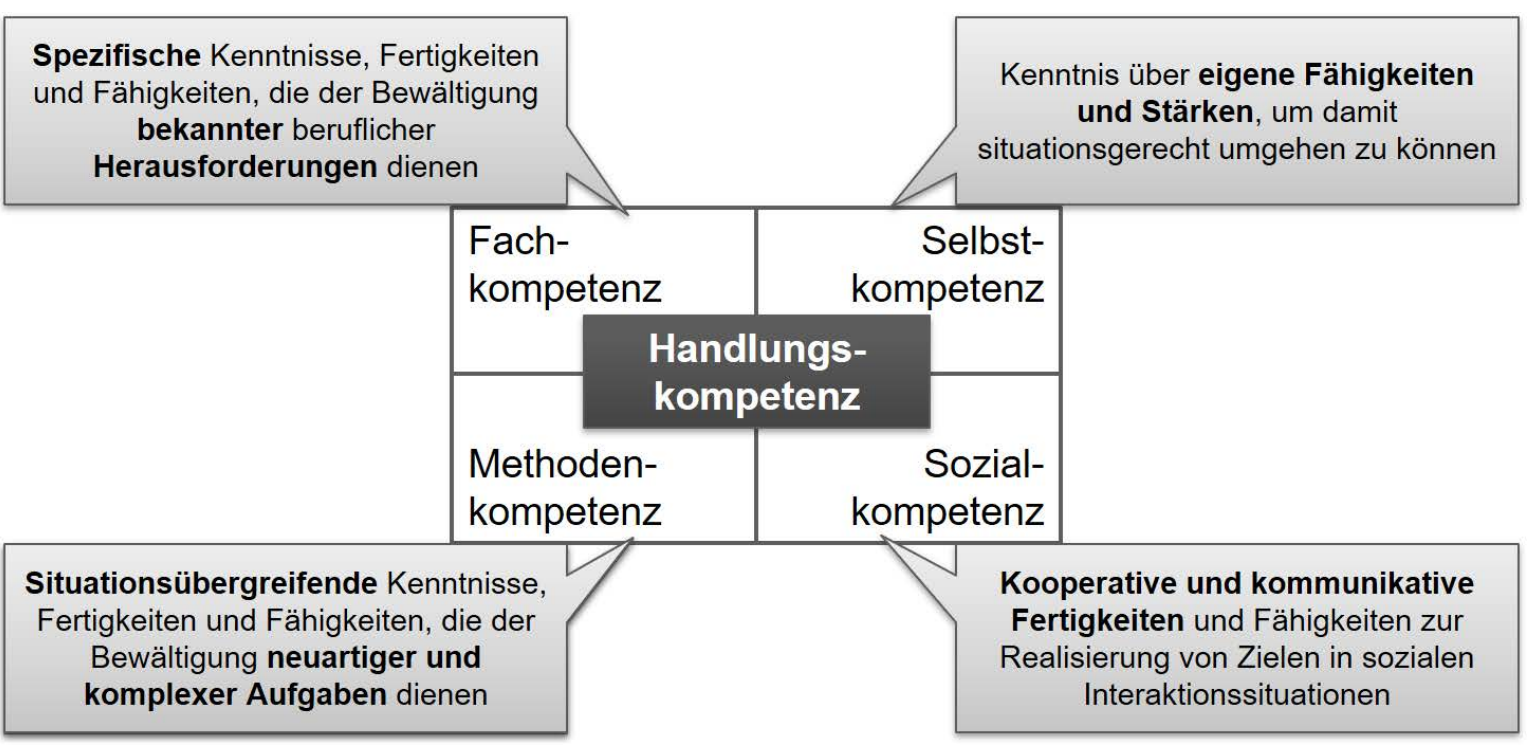

Abbildung 2: Kompetenzklassen beruflicher Handlungskompetenz in Anlehnung an Roth (1971) und North et al. (2018); eigene Darstellung.

Für die Erweiterung beruflicher Handlungskompetenz durch Trainings können zahlreiche Methoden eingesetzt werden. Kauffeld (2016) gibt einen Überblick über die klassischen Trainingsmethoden, die wichtigsten davon sind nachfolgend kurz erläutert:

- Lehrvortrag bzw. Lehrgespräch (interaktive Vortragsform):

Frontale Informationsvermittlung zur Einführung in ein Thema, Wissensvermittlung, Erklärung von Zusammenhängen und Hervorhebung wichtiger Punkte

- Stillarbeit:

Bearbeitung einer Aufgabe durch die Teilnehmenden für sich zur Selbstreflexion, Wissensanwendung oder Lernerfolgskontrolle

- Murmelgruppe:

Angeleiteter Austausch durch Murmeln mit einem oder zwei Sitznachbarn

- Rollenspiel:

Nachstellen einer Anwendungssituation im geschützten Rahmen, um neue Zielverhaltensweisen auszuprobieren bzw. einzuüben

- Gruppenarbeit:

Bearbeitung einer Aufgabe in Kleingruppen mit anschließendem Ergebnisaustausch im Plenum

- Videofeedback:

Systematische Reflexion und Analyse von kurzen Präsentationen oder Rollenspielen anhand einer Videoaufnahme, wodurch sich Teilnehmende auch aus der Außenperspektive betrachten können 
Eine kombinierte Trainingsmethode, die Vortrag, Rollenspiel, Gruppenarbeit und Videofeedback systematisch integriert, stellt das „Behavior Modeling“ dar. Es basiert auf der Theorie des sozialen Lernens von Bandura (1977) und folgt üblicherweise dem in Abbildung 3 dargestellten Ablauf. Nach einer Themeneinführung, werden zusammen mit den Teilnehmenden interaktiv Lernpunkte definiert, deren Wirkung anhand eines Verhaltensmodells aufgezeigt und diskutiert wird. Daraufhin folgt eine Übungseinheit, bei der Teilnehmende die Lernpunkte anwenden und den Erfolg reflektieren können. Insbesondere für die Vermittlung von Sozialkompetenzen (wie z. B. Gesprächsführung oder Konfliktmanagement) ist Behavior Modeling eine sehr effektive Trainingsform (M. J. Burke \& Day, 1986; Taylor, Russ-Eft \& Chan, 2005).

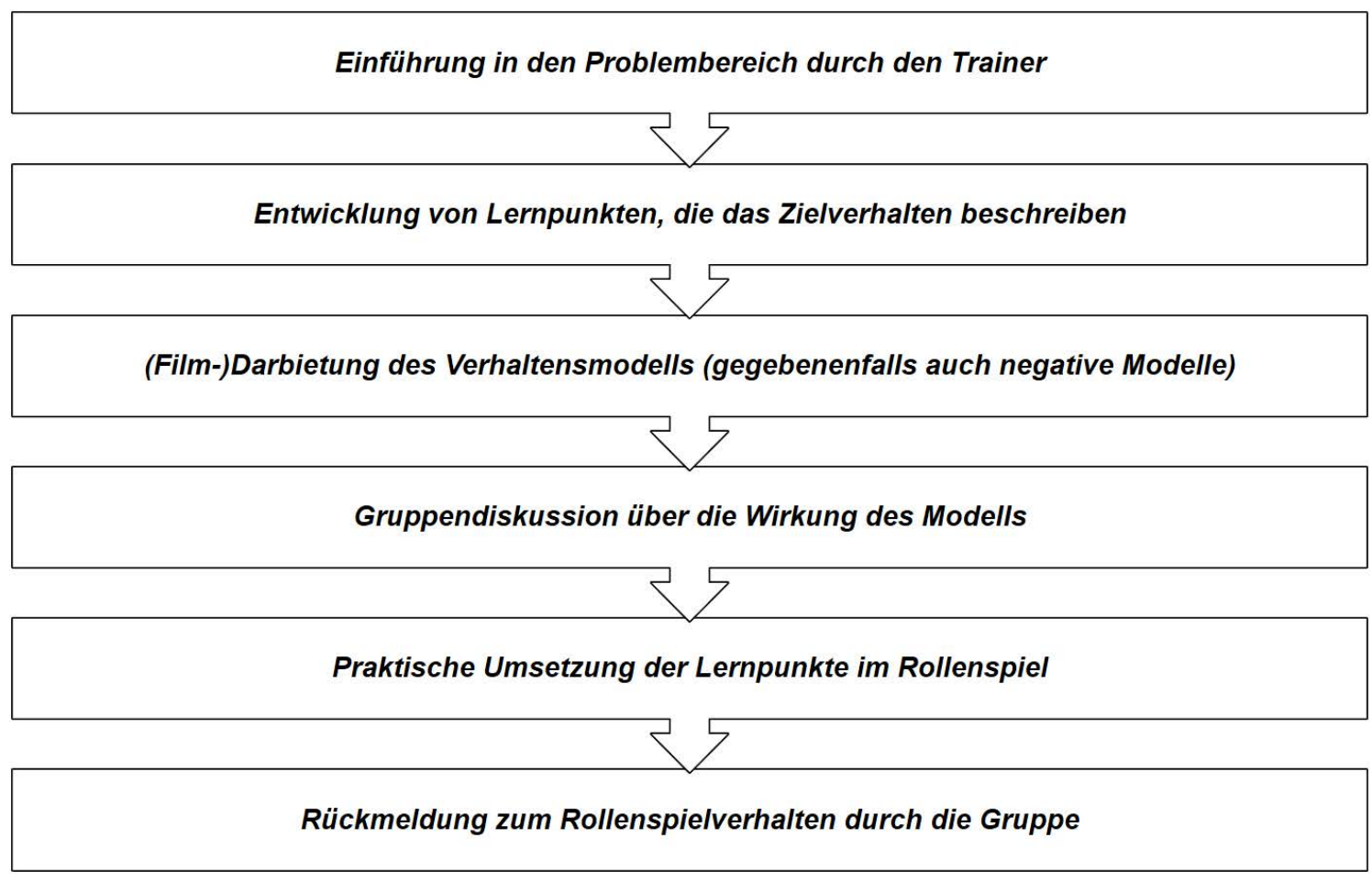

Abbildung 3: Ablauf des Behavior Modeling Trainings in Anlehnung an Taylor et al. (2005); eigene Übersetzung und Darstellung.

Trainings können als Personalentwicklungsmaßnahme für sämtliche Zielgruppen von Mitarbeitenden ausgerichtet werden. In dieser Arbeit wird davon der Ausschnitt der Trainings für Führungskräfte zur Vermittlung interaktionaler Kompetenzen betrachtet. 


\subsection{Trainingswirksamkeit}

Das bis heute einflussreichste Modell der Trainingsforschung für die umfassende Erfassung der Trainingswirksamkeit stammt von Kirkpatrick (1967, 1998). Es empfiehlt eine multidimensionale Betrachtung der Trainingswirksamkeit anhand von vier Dimensionen: 1) Reaktion im Sinne einer subjektiven Zufriedenheits- und Nützlichkeitsbewertung des Trainings, 2) Lernen im Sinne von Kompetenzentwicklung bezüglich Wissen, Einstellung und/oder Verhalten während des Trainings 3) Verhalten im Sinne von Transfer trainierter Kompetenzen in den Arbeitskontext und 4) Ergebnisse im Sinne organisationaler Erfolgskriterien durch verbesserter Arbeitsleistung aufgrund erfolgreichen Transfers. Die Reihenfolge der vier Ebenen entspricht dabei der Zunahme des Trainingsabstandes und der betrieblichen Relevanz. So können die ersten beiden Ebenen als kurzfristige Ergebnisse des unmittelbaren Trainingserfolges betrachtet werden, während die Ebene drei und vier den langfristigen Transfererfolg durch erfolgreiche Anwendung der Trainingsinhalte definieren. In der Trainingsforschung gilt daher Transfer als zentrales Erfolgskriterium der Trainingswirksamkeit (Baldwin et al., 2017; Blume, Ford, Baldwin \& Huang, 2010; Ford et al., 2018; Kauffeld, 2016). Zufriedenheit und Nützlichkeitseinschätzung sowie Kompetenz ergeben erst durch effektive Kompetenzanwendung realen Mehrwert. Wirtschaftliche Ergebnisgrößen auf organisationaler Ebene können resultieren, sind aber nur teilweise durch individuelle Leistung beeinflussbar. Für die konkrete Anwendung des Modells gibt Kauffeld (2016) die in Abbildung 4 dargestellten Beispiele für Messgrößen bzw. Operationalisierungen vor.

\begin{tabular}{|c|c|}
\hline Evaluationsebene & Beispiele zur Messung der Wirksamkeit \\
\hline Reaktion & $\begin{array}{l}\text { - Zufriedenheit der Teilnehmenden } \\
\text { - Nützlichkeit aus Sicht der Teilnehmenden }\end{array}$ \\
\hline Lernen & $\begin{array}{ll}\text { - } & \text { (Wissens-)Tests } \\
\text { - Lernerfolg aus Sicht der Teilnehmenden }\end{array}$ \\
\hline Verhalten bzw. Transfer & $\begin{array}{l}\text { - Transferquantität (Anzahl umgesetzter Schritte) } \\
\text { - } \quad \text { Kransferqualität (durchschnittlicher Umsetzungsgrad) } \\
\text { Grote \& Henschel, 2007) durch Selbst- und bzw. oder Fremdeinschätzung } \\
\text { - Beobachtungsinstrumente wie act4teams (Kauffeld, 2006) }\end{array}$ \\
\hline Resultate & $\begin{array}{l}\text { - Produktivitätskennzahlen } \\
\text { (Umsatz, Gewinn, Kunden, Marktanteile, etc.) } \\
\text { - Anzahl und Umsetzung von betrieblichen Verbesserungsvorschlägen } \\
\text { - } \text { Fluktuation } \\
\text { - Absentismus }\end{array}$ \\
\hline
\end{tabular}

Abbildung 4: Vier Ebenen des Trainingserfolgs und mögliche Operationalisierungen nach Kauffeld (2016, S. 27); eigene Darstellung. 
Das von Kirkpatrick $(1967,1998)$ eingeführte Kategoriesystem für die umfassende Erfassung von Trainingswirksamkeit wurde insbesondere bezüglich der Annahme, dass die vier Ebenen voneinander abhängig bzw. positiv verknüpft sind, vielfach kritisiert. So konnte der Zusammenhang zwischen der Reaktionsebene und den übrigen Ebenen in vielen Studien nicht bestätigt werden. Zumindest die Zufriedenheitseinschätzung kann weder Lern- noch Transfererfolg verlässlich vorhersagen (Arthur, Bennett, Edens \& Bell, 2003; Blume et al., 2010; Kauffeld, 2016). „Der Effekt kann sogar gegenläufig sein: Ein Training, das die Teilnehmer konfrontativ an ihre Grenzen bringt und nicht zur hohen Zufriedenheit der Teilnehmer führt, kann die Teilnehmer zur Reflektion ihres Verhaltens anregen und zu Verhaltensänderungen am Arbeitsplatz führen“ (Kauffeld, Lorenzo \& Weisweiler, 2012). Die Nützlichkeitseinschätzung, als weiteren Aspekt der Reaktionsebene, wird hingegen als geeigneteren Prädiktor für Lern- und Transfererfolg betrachtet (Blume et al., 2010; Kauffeld, 2016; Tharenou, Sakes \& Moore, 2007), wobei metaanalytisch aufgezeigt wurde, dass der Zusammenhang zu Transfer und dessen resultierenden Effekte stärker ist als zu Lernen (Alliger, Tannebaum, Bennett \& Traver, 1997). Das kann durch den Zusammenhang der Reaktionseben zu Anwendungsabsichten und Transfermotivation erklärt werden, da diese Variablen als zentrale Faktoren für Transfererfolg gelten (Bauer, Orvis, Ely \& Surface, 2016; Hughes et al., 2016; Lacerenza et al., 2017; Massenberg, Schulte \& Kauffeld, 2017; Reinhold, Gegenfurtner \& Lewalter, 2018; Sitzmann \& Weinhardt, 2018). Ein weiterer Kritikpunkt an Kirkpatricks Modell ist der schwache Zusammenhang zwischen Lern- und Transferebene (Alliger et al., 1997). Zudem besteht insbesondere auf der Resultatebene bei klassischen monetären Erfolgskennzahlen das Problem nichtkontrollierbarer Kriterienkontamination z. B. aufgrund zeitversetzter Wirkung, Abhängigkeit von Interaktionspartnern oder wirtschaftlicher Entwicklung und der damit verbundenen großen Asymmetrie zwischen Intervention und Kriterium. Während beispielsweise bei Vertriebstrainings der Bezug zu Umsatzzahlen direkt gegeben ist, wirken Führungskräftetrainings nur indirekt darauf ein (Tharenou et al., 2007), wobei sich die Festlegung eines sinnvollen zeitlichen Rahmens, bis sich Transfereffekte in monetären Erfolgskennzahlen niederschlagen, nicht fundiert ableiten lässt. Dennoch ist Kirkpatricks Modell einschlägig für die Evaluationsforschung im Trainingskontext. Einerseits liegt dies wohl darin begründet, dass es der Vielschichtigkeit von Trainingsoutcomes bzw. der Definition von Trainingserfolg Rechnung trägt. Andererseits können durch die Einordnung in ein einheitliches Kategoriesystem Forschungsergebnisse besser abgeglichen werden und somit den Erkenntnisgewinn besser unterstützen. So wurde die Unterscheidung auch in zentralen Studien und Metaanalysen zur Transferforschung herangezogen (siehe auch Abschnitt 3.1 ab Seite 31). Zur Einordnung in 
den Forschungskontext und Strukturierung wird das Modell zur Trainingsevaluation von Kirkpatrick $(1967,1998)$ auch in dieser Evaluationserhebung verwendet.

Felfe und Franke (2014) haben Kirkpatricks Modell auf Führungskräftetrainings übertragen (siehe Abbildung 5). Darin ist zusammengefasst, welche Fragen eine Evaluation auf den jeweiligen Ebenen beantworten soll.

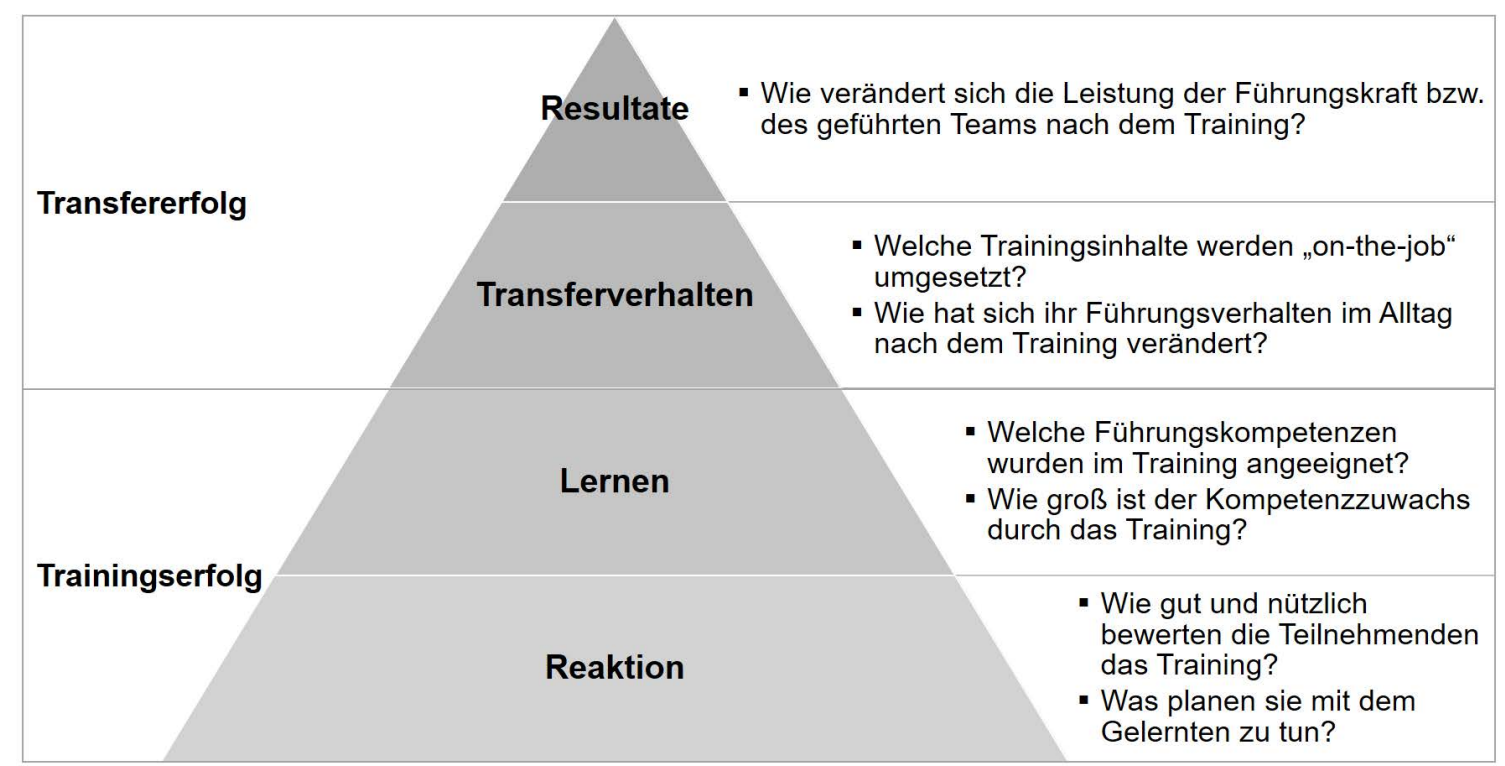

Abbildung 5: Kirkpatricks Modell der Trainingsevaluation zur Wirksamkeitsüberprüfung von Führungskräftetrainings nach Felfe und Franke (2014, S. 44); eigene Darstellung.

Der unmittelbare Erfolg von Führungskräftetrainings wird demnach durch Zufriedenheit, Nützlichkeit und motivationalen Reaktionen sowie durch erlernte Führungskompetenzen definiert. Der langfristige Transfererfolg ist ebenfalls zweistufig operationalisiert: Einerseits durch die Überprüfung, inwiefern sich das Führungsverhalten durch das Training verändert hat; andererseits durch die Überprüfung der daraus resultierenden Effekte, also ob die Veränderung des Verhaltens als positiv wahrgenommen wird und damit die Teamleistung fördert. So kann das diskutierte Problem von Kirkpatricks Resultatebene aufgrund der großen Asymmetrie zwischen Intervention und Kriterium durch die Anwendungsempfehlung von Felfe und Franke (2014) gelöst werden. 


\subsection{Trainingstransfer}

Nach dem in Abschnitt 2.2 ab Seite 12 beschriebenen Modell der Trainingswirksamkeit von Kirkpatrick $(1967,1998)$ ist Transfererfolg durch die Anwendung trainierter Kompetenzen und der daraus resultierenden Verbesserung der Arbeitsleistung definiert. Trainingstransfer (auch als "Lerntransfer" bezeichnet) wird daher als entscheidendes Maß der Trainingswirksamkeit betrachtet (Baldwin et al., 2017; Blume et al., 2010; Felfe \& Franke, 2014; Kauffeld, 2016) und wird im Rahmen dieser Arbeit als die erfolgreiche Übertragung und Anwendung der in Trainings (Lernfeld) erworben Kompetenzen auf den Arbeitskontext (Funktionsfeld) verstanden. Das impliziert eine effektive Übertragung auf Herausforderungen des Arbeitsalltags (Generalisierung) und kontinuierliche Anwendung (Aufrechterhaltung) von trainierten Kompetenzen (Baldwin \& Ford, 1988). Als Trainingstransfer oder Lerntransfer, kann auch die Übertragungsleistung beruflicher Kompetenzen aus dem Arbeitskontext auf ein Training bzw. Lernfeld verstanden werden (Lobato, 2003, 2006). Auch wenn dieser Transferprozess für erfolgreiche Kompetenzentwicklung durch effiziente Anknüpfung von neu Erlerntem wichtig sein kann, liegt der Fokus dieser Arbeit auf dem Transferprozess nach Führungskräftetrainings.

Nach Mandl, Prenzel und Gräsel (1992), werden verschiedene Formen von Trainingstransfer unterschieden: Basierend auf der Taxonomie von Gagne (1965) wird einerseits abhängig von der Ähnlichkeit zwischen Lern- und Funktionsfeld die Anwendung erworbener Kompetenzen auf Trainingsniveau („lateral“ bzw. „naher Transfer“) von der Übertragung auf komplexere Herausforderungen mit weiterem Kompetenzaufbau („vertikaler“ bzw. „weiter Transfer“) abgegrenzt (siehe auch Hasselhorn \& Mähler, 2000) ${ }^{4}$. Bei Trainingstransfer von interaktionalen Führungskompetenzen handelt es sich meist um Anwendung auf komplexeren Anforderungsniveaus. Somit geht es bei der Wirksamkeitsüberprüfung von Führungskräftetrainings vorwiegend um vertikalen Transfer. Da wie bereits erläutert, die Anforderungen an Transferleistung mit der Transferdistanz (Grad der Unterschiede zwischen Lern- und Funktionsfeld) wächst, ist die Transferförderung bei Führungskräftetrainings besonders wichtig. Andererseits wird Transfer nach Erfolg differenziert. Da die Übertragung des Gelernten nicht zwangsläufig intendierte Verbesserungen der Arbeitsleistung bewirkt, was als Transfer i. e. S. oder „positiver Transfer“ definiert ist, werden davon zwei weniger erfolgreiche Transferformen abgegrenzt: Bleibt eine positive Trainingswirkung im Funktionsfeld aus (z. B. aufgrund fehlender Alltagsrelevanz

${ }^{4}$ Es gibt noch weitere Kategorisierungen von Transfer nach Transferdistanz, beispielsweise von Eraut (2004): einfache Übertragung, Situative Anpassung, Problemlösung oder von D. L. Schwartz, Bransford und Sears (2005) wonach Transferleistung anhand von den zwei Dimensionen „Wirksamkeit" und „Innovation“ bei Anwendung des Gelernten definiert werden kann. 
der Trainingsinhalte) wird von „Nulltransfer“ gesprochen. Bewirkt die Kompetenzanwendung sogar eine Verschlechterung (z. B. aufgrund fehlender Authentizität), wird dies als „negativer Transfer“ bezeichnet. Ausbleibender Trainingstransfer. i. e. S. (bzw. Null- und negativer Transfer) stellt das seit Jahrzehnten beklagte Transferproblem dar.

Die prozessorientierte Transferforschung zeigt, welche Determinanten für positiven Transfer relevant sind. Nach dem Basismodell von Baldwin und Ford (1988) werden die Wirkmechanismen für Generalisierung und Aufrechterhaltung von trainierten Kompetenzen durch Wechselwirkungen zwischen Merkmalen von Teilnehmenden (Fähigkeit, Persönlichkeit und Motivation), Trainingsgestaltung (Lernprinzipien basierend auf fundierten Lerntheorien, Inhaltsrelevanz und zeitliche Abfolge) und Arbeitsumgebung (soziale Unterstützung und Anwendungsmöglichkeiten) definiert. Dabei gilt Kompetenzentwicklung als zentraler Mediator. Jüngere Forschung rückt motivationale und situative Aspekte ins Zentrum des Transferprozesses. Dabei gewinnt der selbstverstärkende Kreislauf (erstes Transferverhalten, dessen positive Folgen und ihre Rückwirkung auf weiteren Kompetenzaufbau, Transfermotivation und -möglichkeit) für nachhaltigen Transfererfolg an Beachtung (Blume et al., 2010).

Das Lerntransfer-System-Inventar (LTSI) von Holton, Bates und Ruona (2000) fasst empirisch überprüfte Transferdeterminanten zusammen. Es ist das bisher einzige global validierte Erhebungsinstrument für prozessorientierte Transferforschung. Für den deutschsprachigen Raum gibt es das Deutsche Lerntransfer-System-Inventar (Kauffeld, Bates, Holton \& Müller, 2008). Das LTSI-Modell (siehe Abschnitt 3.2.2 ab Seite 73) eignet sich als Basis zur Überprüfung und Förderung von Transferprozessen (Seeg \& Schütz, 2019).

\subsection{Trainingsevaluation}

Der Begriff Evaluation umfasst im weitesten Sinne die Bewertung von Objekten, also von Produkten und Programmen bzw. Prozessen (Suchman, 1967). Im Kontext der Personalentwicklung beziehen sich Evaluationen auf die Bewertung von Entwicklungsmaßnahmen, so umfasst der hier verwendete Evaluationsbegriff „sämtliche Aktivitäten zur Qualitäts- und Erfolgskontrolle einer Personalentwicklungsmaßnahme: Sie dient der Bewertung der inhaltlichen und didaktischen Konzeption, der Durchführung, der Wirksamkeit und der Wirtschaftlichkeit des Programms" (Solga, 2011a, S. 369). Nach diesem Verständnis umfasst Evaluation sowohl Wirksamkeits-, als auch Wirkungsüberprüfungen, um nicht nur der Frage begegnen zu können, ob eine Maßnahme wirksam ist, sondern auch zu erklären warum das Ergebnis zustande gekommen ist und wie die Maßnahmeneffektivität optimiert werden kann. Auch auf dieser Betrachtungsebene gibt es 
Evaluationsmodelle und Theorien mit unterschiedlicher Ausrichtung. Eine übergeordnete Differenzierung bzw. Spezifizierung grenzt die „Evaluationsforschung“ im Sinne wissenschaftlich fundierter Evaluation von alltäglicher Bewertung ab (siehe auch Chen, 1990): „Evaluationsforschung integriert wissenschaftliche Strategien, Methoden und Modelle um Bewertungen durchzuführen“ (Wittmann, 2009, S. 60). Da es im Rahmen der vorliegenden Arbeit grundsätzlich um wissenschaftliche Evaluationen geht, wird diese Spezifizierung im hier verwendeten Begriff „Evaluation“ impliziert. Während der Begriff „Evaluationsforschung“ für die Bezeichnung der Forschungsdisziplin verwendet wird, um Doppeldeutigkeit zu vermeiden. Da die Evaluationsforschung ein interdisziplinäres Feld ist, gibt es zahlreiche Ansätze und Definitionen für Evaluation mit unterschiedlicher Schwerpunktsetzung und Ausrichtung. Einen Überblick über zentrale Evaluationsmodelle gibt beispielsweise Wittmann (2009), der sich an der Einteilung nach Bewertungs-, Methoden- und Anwendungsfokus von Alkin (2004) orientiert. Nachfolgend sind die zentralen Modelle dieser drei Definitionsperspektiven beschrieben.

Die zentrale Theorie, die den Bewertungsfokus von Evaluationen definiert stammt von Scriven (1991), aus der die grundlegende Unterscheidung von Evaluationsperspektiven in "formative“ und „summative“ Evaluation hervor geht. Summative Evaluationsmodelle beziehen sich auf ergebnisorientierte Wirksamkeitsüberprüfungen zur retrospektiven Bewertung, ob bzw. inwiefern eine Maßnahme den erzielten Nutzen auf organisationaler (Makro-)Ebene generieren konnte. Formative Evaluationsdefinitionen betrachten durch prozessorientierte Wirkungsüberprüfung prospektiv dahinterliegende Wirkmechanismen, die für die jeweilige Wirkung auf individueller (Mikro-)Ebene ursächlich sind. In wirtschaftswissenschaftlichen Betrachtungen von Personalentwicklungsevaluationen steht die Wirksamkeitsanalyse im Vordergrund, wobei insbesondere Modelle zur Bewertung des monetären Nutzens herangezogen werden (Kellner, 2006; Phillips \& Schirmer, 2008). Entsprechend wird hier Evaluation üblicherweise unter dem Begriff „Bildungscontrolling" betrachtet. Wirtschaftswissenschaftliche Modelle, die formative Betrachtungen zu summativen Bewertungen ergänzen, reihen die Evaluation formal als einen Arbeitsschritt retrospektiv erst nach erfolgter Durchführung in den Personalentwicklungsprozess ein (z. B. Funktionszyklus systematischer Personalentwicklung von Becker, 2011). Pädagogische bzw. erziehungswissenschaftliche Evaluationsforschung in der Erwachsenenbildung bezieht sich eher auf die formative Perspektive durch qualitative Bewertungen (insbesondere durch Beobachtung) von Lehr-Lern-Prozessen und Wechselwirkungen zwischen Teilnehmenden und Intervention (siehe z. B. Lernendenorientierte Qualitätstestierung in der Weiterbildung). Es gibt aber auch dort ergebnisbezogene summative Betrachtungen wobei eher die Qualifikation bzw. der Lernerfolg statt monetärer 
Aspekte als Ergebniskriterium herangezogen werden. Psychologische Evaluationsforschung in der Personalentwicklung betrachtet auf der Ergebnisseite vor allem Kompetenzentwicklung und erzielte Verhaltensänderungen. Sie basiert jedoch vorwiegend auf Modellen, die summative und formative Evaluationsansätze integrieren, wobei die formative Berücksichtigung prozessbezogener Betrachtungen als Erfolgsfaktor für nachhaltigen Transfer betrachtet werden (Hochholdinger, Rowold \& Schaper, 2008; Kauffeld, 2016; Solga, 2011a)

Evaluationsmodelle des Methodenfokus nach Alkin (2004) befassen sich mit der Operationalisierung und Messung von Evaluationskriterien. Sie entstammen insbesondere aus dem Bereich Forschungsmethoden und sind vorwiegend auf die summative Bewertung von Programmen bzw. Interventionen (sogenannte „Programmevaluation“) ausgerichtet. Erste Ansätze gehen auf Brodgen (1949) mit seiner Formel zur Berechnung der Kosten-Nutzen-Relation von psychologischen Interventionen bzw. Trainings zurück, die von Cronbach und Gleser optimiert wurde (siehe Brodgen-Cronbach-Gleser-Gleichung; Brodgen, 1949; Cronbach \& Gleser, 1965). Das bis heute einflussreichste summative Evaluationsmodell zur Operationalisierung der Wirksamkeit von Personalentwicklungsmaßnahmen ist das bereits im Abschnitt 2.2 ab Seite 12 zur Trainingswirksamkeit vorgestellte Modell von Kirkpatrick $(1967,1998)$ mit den vier Ebenen Reaktion, Lernen, Transferverhalten und Resultate.

Als Basismodell des Anwendungsfokus der Evaluation gilt das "CIPP-Modell“ von Stufflebeam (1983, 2004), das in Abbildung 6 dargestellt ist. Namengebend sind dabei die Anfangsbuchstaben der vier übergeordneten Evaluationsphasen Kontext- (C), Input- (I), Prozess- (P) und Produktevaluation (P). Die empfohlenen Evaluationsschritte beziehen sich dabei auf eine systematische formative bzw. summative Bewertung von Zieldefinition, Planung, Umsetzung und Ergebnissen unter Anwendung der Kernwerte bzw. Evaluationsstandards des Joint Committee on Standards for Educational Evaluation (2011) $)^{5}$. Demnach sollen sich Evaluationen unabhängig vom jeweiligen Bewertungsgegenstand an vier Standards orientieren, was durch eine abschließende Metaevaluation zu überprüfen ist: Nützlichkeit, Durchführbarkeit, Fairness und Genauigkeit.

\footnotetext{
${ }^{5}$ Für den deutschsprachigen Raum stellt die Gesellschaft für Evaluation (DeGEval, 2016) darauf basierende Standards und Checklisten zur Qualitätssicherung von Evaluationen zur Verfügung (https://www.degeval.org/degeval-standards/download/).
} 


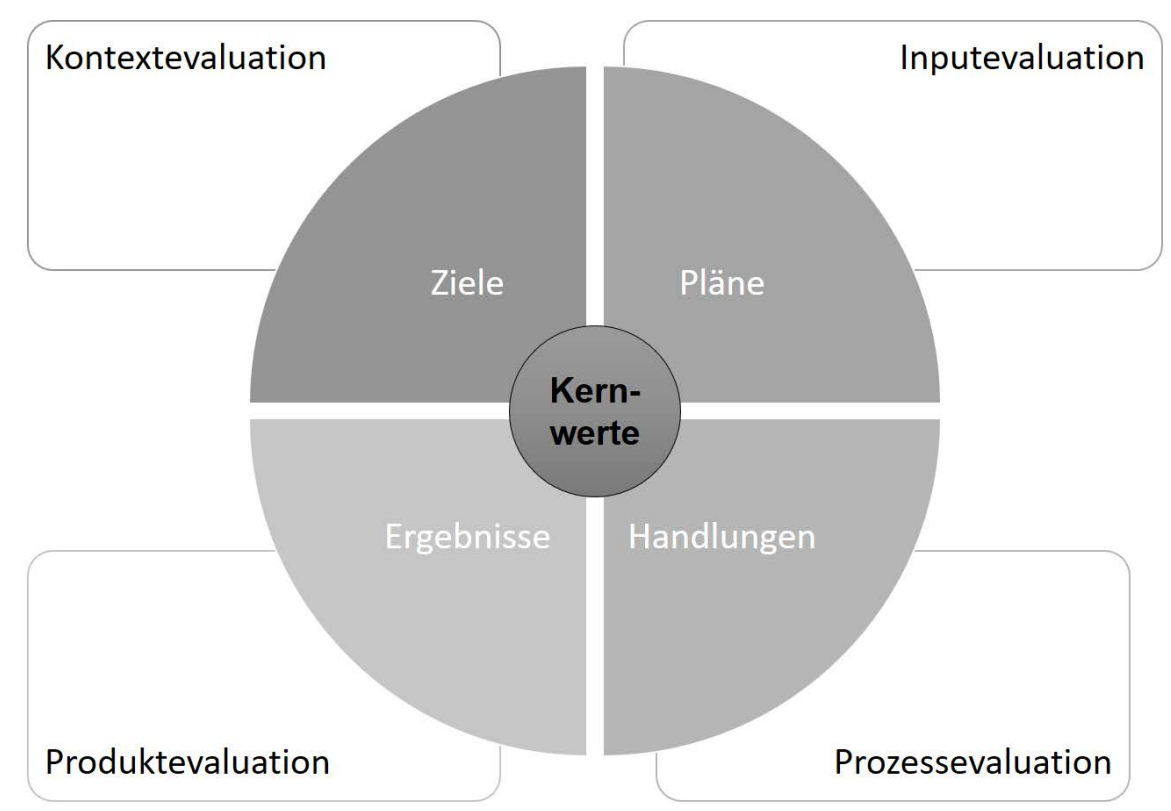

Abbildung 6: CIPP-Evaluationsmodell von Stufflebeam (1983, 2004); eigene Übersetzung und Darstellung.

Durch diese vier Phasen kann der gesamte Interventionslebenszyklus prozessbegleitend evaluiert werden, es können aber auch nur Teilevaluationen anhand einzelner Phasen durchgeführt werden. Im Sinne einer umfassenden Qualitätssicherung von Interventionen, wird die Nutzung und Integration aller Evaluationsphasen empfohlen, da sie letztlich auch systematisch aufeinander aufbauen. Jeweilige Aufgabenbeispiele der vier Phasen sind nachfolgend für summative (retrospektive Anwendung für Nutzenbewertung) und formative (prospektive Anwendung für Qualitätssicherung) Evaluationsfunktionen in das Modell eingeordnet, um dessen Verwendung zu veranschaulichen:

- Kontextevaluation:

- Formativ: What needs to be done?

Identifikation benötigter Interventionen und Programmziele anhand von Bedarfs- und Möglichkeitsanalysen

- Summativ: Were assessed beneficiary needs addressed? Abgleich von Programmzielen mit Bedürfnissen, Herausforderungen und Möglichkeiten

- Inputevaluation:

- Formativ: How should it be done?

Überprüfung des Konzeptplans und Erfassung möglicher Alternativen zur Zielerreichung mit den verfügbaren Ressourcen

- Summativ: Were the needs addressed by a sound, responsive plan? Vergleich von geplantem Konzept mit alternativen Ansätzen zur Zielerreichung unter Berücksichtigung von Aufwand-Nutzen-Relation 
- Prozessevaluation:

- Formativ: Is it being done?

Monitoring und Beurteilung der Konzeptumsetzung

- Summativ: Was the program's plan effectively implemented?

Abgleich von geplantem Konzept und tatsächlicher Programmdurchführung

\section{- Produktevaluation:}

- Formativ: Is it succeeding?

Überprüfung der Notwendigkeit einer Programmfortführung, -modifikation, -beibehaltung oder -beendigung durch Bewertung der Haupt- und Nebeneffekte unter Berücksichtigung von Aufwand-Nutzen-Relation sowie praktischer Relevanz

- Summativ: Did the program succeed?

Überprüfung, ob Programmziele erreicht wurden

Dieses Prozessmodell kann wie aufgezeigt für summative, formative und kombinierte Evaluationen genutzt werden und weist durch konkrete Handlungsempfehlungen für die Durchführung der einzelnen Evaluationsphasen eine klare Praxisorientierung auf. Es wurde stetig weiterentwickelt und insbesondere die Produktevaluation weiter ausdifferenziert, um der Bedeutung langfristiger Programmergebnisse Rechnung zu tragen besonders bezüglich Transfer (Stufflebeam \& Zhang, 2017). Checklisten zur Umsetzung und Qualitätssicherung von Programmevaluationen nach dem Modell von Stufflebeam sind auf der Homepage "The Evaluation Center“ (https://wmich.edu/evaluation/checklists) frei verfügbar. Auch wenn diese Checklisten sehr konkret einzelne Prozessschritte auflisten (teilweise so sehr, dass ein Überblick schwerfällt) bleibt bei vielen Schritten offen, wie diese sinnvoll zur Evaluation von Personalentwicklungsmaßnahmen umgesetzt werden können.

Ein Evaluationsmodell, das Bewertungs-, Methoden- und Anwendungsfokus integriert und auf den beschriebenen Prozess formaler Personalentwicklung (siehe Seite 8) anwendet, stellt das Evaluationsmodell von Solga (2011a) ${ }^{6}$ aus Abbildung 7 dar. Darin sind summative und formative Evaluationsfunktionen zur Qualitätsüberprüfung und -sicherung sinnvoll kombiniert sowie die Evaluationsschritte anwendungsorientiert mit Um-

${ }^{6}$ Es gibt ähnliche Evaluationsmodelle für Personalentwicklungsmaßnahmen, die einige Eigenschaften teilen (z. B. von Becker, 2011; Grohmann \& Kauffeld, 2013; Will, Winteler \& Krapp, 1987). Aufgrund der übersichtlichen und praxisorientierten Integration von Handlungsempfehlungen zur summativen und formativen Evaluation anhand des Prozessmodells der Personalentwicklung, ist es anderen Modellen bezüglich der Passung auf das vorliegende Evaluationsprojekt überlegen. 
setzungsbeispielen auch zur Operationalisierung in den Gesamtprozess integriert. Erläuterungen zu den Bestandteilen der Maßnahmengestaltung und ihrer Evaluation finden sich auch im sogenannten Funktionszyklus systematischer Personalentwicklung von Becker (2011), der im Personalmanagement etwas bekannter ist. Solga (2011a) stellt in seinem Modell jedoch das prozessbegleitende Vorgehen und die Bedeutung der Evaluation klarer heraus statt diese als Phase zwischen Durchführung und Transfersicherung einzureihen, was insbesondere für ein systematisches Transfermanagement von Bedeutung ist. Daher dient dieses Modell als konzeptionelles Grundgerüst der vorliegenden Arbeit und wird nachfolgend beschrieben.

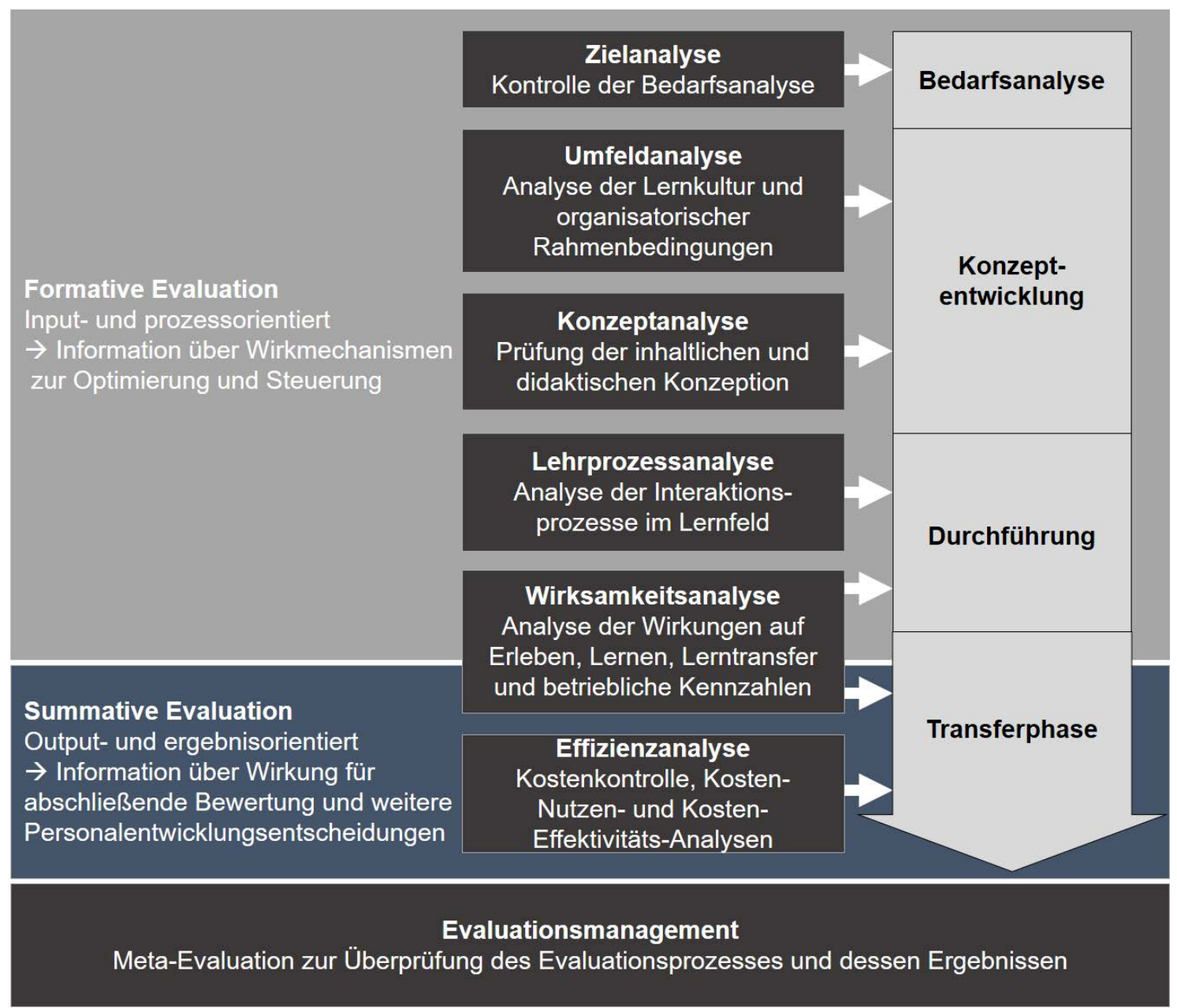

Abbildung 7: Prozess einer systematischen Trainingsevaluation nach Solga (2011a, S. 373); eigene Darstellung.

Die rechts abgebildete Prozesskette spiegelt die Phasen des Personalentwicklungsprozesses wider (siehe auch Seeg, 2020, S. 170 ff.): Bedarfsanalyse, Konzeptentwicklung, Durchführung und Transfersicherung. In der Mitte sind die dazugehörigen Aufgaben der Evaluation zur kontinuierlichen Erfolgskontrolle dargestellt. Demnach wird die prozessbegleitende Evaluation in die Analyse der Zielbestimmung, Rahmenbe- 
dingungen, Trainingsentwicklung und -durchführung, sowie der Wirksamkeit und Effizienz unterteilt. Auf der linken Seite ist beschrieben, welchem Ziel die einzelnen Aufgaben der Evaluation dienen. Zu einer vollständigen Evaluation gehört einerseits die formative Betrachtung zur Identifikation der Wirkmechanismen und Optimierungsmöglichkeiten in der Maßnahmengestaltung. Andererseits soll eine Evaluation prüfen, ob Personalentwicklungsmaßnahmen die erzielte Wirkung zeigen. Durch eine Kombination kann nicht nur überprüft werden, ob eine Maßnahme nachhaltig wirksam ist, sondern auch wie der jeweilige Wirkungsgrad zustande gekommen ist. Entgegen dem CIPP-Modell wird in diesem Modell die Kontextevaluation in Ziel- und Umfeldanalyse sowie die Produktevaluation in Wirksamkeits- und Effizienzanalyse unterteilt. Zudem wird eine klare Ausrichtung der jeweiligen Evaluationsschritte entsprechend ihres Beitrags für den Gesamtprozess auf formative bzw. summative Bewertung vorgegeben.

Ziel-, Umfeld-, Konzept- und Lehrprozessanalysen können die Wirkmechanismen von Trainings aufzeigen. Für diese Analysen können beispielsweise Fragebogenverfahren wie das deutsche Lerntransfer-System-Inventar (GLTSI; Bates, Kauffeld \& Holton, 2008; Kauffeld et al., 2008), Beobachtungsverfahren der Interaktionsprozesse im Lernfeld oder ein inhaltlicher Abgleich von Bedarf, Konzept und Durchführung basierend auf Interviews oder Dokumentenanalyse genutzt werden.

Für Wirksamkeitsanalysen kann das Modell der Trainingsevaluation von Kirkpatrick (1967, 1998) herangezogen werden, das vier Ebenen des Maßnahmenerfolgs für umfassende und strukturierte Erfolgsmessungen empfiehlt (siehe Seite 12). Neben der Hauptaufgabe der summativen Erfassung des Wirkungsgrades, kann die Betrachtung der vier Wirkebenen auch formativ Aufschluss über dahinterliegende Wirkmechanismen geben - insbesondere durch Inhalte der Reaktionsebene und Zusammenhänge zwischen den vier Ebenen. In der Praxis wird die Trainingswirksamkeit vorwiegend anhand subjektiver Reaktionsbewertungen, auch häufig „Happiness-Index“ genannt, erfasst (z. B. mittels sogenannter „Happy-Sheets“). Wesentlich seltener erfolgt eine Überprüfung des Lernerfolgs und nur vereinzelt die des Transfers oder der organisationalen Ergebnisgrößen (Buren \& Erskine, 2002; Kaiser \& Curphy, 2013). Da konfundierende Effekte die Wirksamkeitsprüfung anhand des organisationalen Ergebnisses erschweren und eindeutige Messgrößen kaum zulassen (z. B. Rosenstiel, Molt, Rüttinger \& Wissner, 2005), ist unter Aufwand-Nutzen-Aspekten die geringe Zahl der Wirksamkeitsprüfungen auf der vierten Ebene zu rechtfertigen. Allerdings greifen Wirksamkeitsprüfungen auf den ersten beiden Ebenen für eine Aussage über die Trainingseffektivität zu kurz, denn das entscheidende Erfolgskriterium für Trainingsmaßnahmen ist der Transfer des Gelernten in den Arbeitsalltag (Ford et al., 2018; Kauffeld, 2016; Schaper, 2011). Ein validiertes Fra- 
gebogenverfahren zur ökonomischen Messung des Trainingserfolgs auf allen vier Ebenen ist der Questionnaire for Professional Training Evaluation (Q4TE; Grohmann \& Kauffeld, 2013), durch das Teilnehmende nach dem Training anhand von 22 Aussagen ihre prozentuale Zustimmung angeben. Entsprechend können ausschließlich direkte Veränderungsmessungen erfasst werden (z. B. „Durch das Training habe ich viel gelernt"). Ein Abgleich des Wissens- und Kompetenzstandes vor und nach dem Training, eine sogenannte indirekte Veränderungsmessung, ist durch diese reine Nachbefragung nicht zu erbringen. Zwar sind direkte Veränderungsmessungen besonders ökonomisch und enthalten Erfolgsbewertungen, was auch Aussagen bezüglich positivem (vs. negativem) Transfer erleichtert. Allerdings besitzen direkte Veränderungsmessungen durch die retrospektive subjektive Einschätzung verstärkt Verzerrungsgefahren beispielsweise aufgrund sozial erwünschtem Antwortverhalten oder Sympathieempfinden gegenüber den Trainierenden. Daher sind für aussagekräftige Ergebnisse bezüglich Lern- und Transfererfolg indirekten Veränderungsmessungen mit Vorher-Nachher-Messungen reinen Nachher-Messungen überlegen. Wobei eine Kombination mit direkten Veränderungsmessungen empfohlen wird (Bortz \& Döring, 2009; Krampen \& Hank, 2008; Lutz \& Grawe, 2007). Zudem sollte bei Trainingsevaluationen berücksichtigt werden, dass der Transfererfolg erst nach einer ausreichenden Transferzeit sinnvoll erfasst werden kann (Arthur et al., 2003; Blume, Ford, Surface \& Olenick, 2019; Ford et al., 2018). Weitere Hinweise, die bei Evaluationen zu berücksichtigen sind, hat Kauffeld (2016) ab Seite 117 zusammengefasst.

In der Theorie ist im Rahmen von Effizienzanalysen auch eine Kostennutzenberechnung von Personalentwicklungsmaßnahmen Bestandteil der Evaluation. Dadurch ist es insbesondere bei regelmäßig durchgeführten Programmen möglich, wie bei anderen wirtschaftlichen Investitionen, den Return on Investment zu ermitteln. Empfehlungen für die Berechnung eines monetären Nutzens von Personalentwicklungsmaßnahmen geben beispielsweise Kauffeld (2016), Phillips und Schirmer (2008) oder auch Kellner (2006). Allerdings sind trotz elaborierter Formeln exakte Gewinn- oder Nutzenberechnungen von Personalentwicklungsmaßnahmen aus verschiedenen Gründen meist zu komplex (z. B. Rückführbarkeit von Veränderungen auf einzelne Maßnahmen oft nicht möglich, Abhängigkeit individueller persönlicher und situativer Rahmenbedingungen der Anwendung von Lernzielen, schwierige monetäre Bewertung von Verhaltensänderungen, zeitversetzte Wirkung aufgrund benötigter Transferphasen; siehe auch Kauffeld, 2016). Dennoch konnten Studien durch Annäherungen an Rentabilitätsberechnungen Abschätzungen eines Returns on Investment liefern. Diese zeigen, dass sich transferförderlich gestaltete Trainingsmaßnahmen langfristig auszahlen (Bassi \& McMurrer, 2007; Cascio \& Aguinis, 2005; Kraiger, 2002; Tharenou et al., 2007). 
Neben diesen beschriebenen Evaluationsmodellen gibt es noch zahlreiche weitere Modelle für Trainingsevaluationen, die in einem aktuellen Review von Perez-Soltero et al. (2019) zusammengefasst und systematisiert dargestellt sind. Das Modell von Solga zeichnet sich insbesondere durch seinen prozessbegleitenden Ansatz zur Überprüfung formeller Personalentwicklungsmaßnahmen aus, der bereits die Planungsphase von Personalentwicklungsmaßnahmen berücksichtigt. Zudem bietet es eine umfassende und praxistaugliche Integration bewährter Basismodelle für die systematische Überprüfung von Wirksamkeit und Wirkweise. Dadurch kann gezielt überprüft werden, inwiefern Maßnahmen auf strategische Unternehmensziele und -bedarfe in Konzeption und Durchführung ausgerichtet sind und ob die intendierte Kompetenzerweiterung im Arbeitskontext ankommt. Somit wird das Evaluationsmodell als geeignete Grundlage für Qualitätsmanagement im Trainingsbereich und damit für das Transfermanagement erachtet. Das Vorgehen des vorliegenden Forschungsprojektes orientiert sich daher an Theorien und Methoden wissenschaftlicher Evaluation, die in das Evaluationsmodell von Solga (2011a) integriert und gezielt auf Transfermanagement ausgerichtet werden. Im Rahmen dieser Arbeit werden davon die ergebnisorientierte summative und die prozessorientierte formative Wirksamkeitsanalyse und ihre theoretischen und praktischen Implikationen beschrieben.

\subsection{Transfermanagement}

Transfermanagement umfasst sämtliche Aktivitäten zur gezielten Förderung des Trainingstransfers (Lemke, 1995; Solga, 2011b) und somit zur Lösung des Transferproblems. Befunde der ergebnisorientierten Transferforschung legen nahe, dass sich Transfermanagementaktivitäten lohnen (siehe Abschnitt 3.1 ab Seite 31).

Grundsätzlich gibt es zwei situative Stellschrauben zur Förderung transferrelevanter Teilnehmendeneigenschaften und der darauf basierenden individuellen Transferleistung: Transferförderliche Trainingsgestaltung und Arbeitsumgebung. Doch um zu wissen, wie diese Stellschrauben „gezielt“ eingesetzt werden können, ist eine Identifikation zentraler Faktoren individueller Transferleistung erforderlich. Die prozessorientierte Transferforschung liefert dafür inzwischen eine große Zahl potenzieller Transferfaktoren. Das von Holton et al. (2000) entwickelte Lerntransfer-System-Inventar (LTSI) fasst daraus die 16 wesentlichen Faktoren zusammen, die neben dem zentralen Prädiktor Kompetenzentwicklung bzw. Lernen, für Transferleistung relevant sein können. Allerdings erschwert die Variablenzahl mit ihren Wechselwirkungen und unterschiedlichen Effekten über den Transferprozess hinweg eine praxistaugliche Ableitung zielgerichteter Transfermanagementmaßnahmen. Bisherige Forschungsbefunde zeigen zudem auf, dass nur ein Teil der LTSI-Faktoren signifikante Zusammenhänge mit Transferkriterien aufweisen 
und zusammen auch nur ein Teil der Transfervarianz aufklären können. Entsprechend scheinen die integrativen Transfermodelle für die praktische Anwendung zu komplex zu sein und können die Realität dennoch nicht vollständig abdecken (siehe Abschnitt 3.2 ab Seite 43). In der vorliegenden Arbeit wird diesem Problem mit einer theoriegeleiteten Fokussierung auf den Kernprozess bzw. auf die direkten Transferprädiktoren begegnet. Eine Basistheorie zur individuellen Leistung, definiert diese als direkte Funktion einer multiplikativen Verknüpfung von Fähigkeit, Motivation und Möglichkeit (Blumberg \& Pringle, 1982; Bühler \& Siegen, 1999; Campbell, 1990; Cromwell \& Kolb, 2004; Dalal, Bhave \& Fiset, 2014). Überträgt man diese Formel auf Transferleistung, können Kompetenz (als Basisfähigkeit für eine Anwendung erworbener Kompetenzen), Transfermotivation und -möglichkeit (inhaltlich und zeitlich) als direkte Transferfaktoren identifiziert werden 7 . Im Rahmen dieser Arbeit werden diese als „primäre Transferdeterminanten“ bezeichnet. Die übrigen fundierten Transferfaktoren der Teilnehmendeneigenschaften, Trainingsgestaltung und Arbeitsumgebung werden entsprechend als distalere „sekundäre Transferdeterminanten“ definiert, da angenommen wird, dass ihre Transferwirkung weitestgehend durch die primären Determinanten mediiert wird (Kodwani \& Prashar, 2019b; Reinhold et al., 2018; Sahoo \& Mishra, 2019). Dabei wird weiter angenommen, dass die transferförderliche Trainingsgestaltung die wichtigste sekundäre Transferdeterminante und damit auch wichtigste Stellschraube des Transfermanagements ist. Einerseits, weil darin eine Ausrichtung auf Trainingsbedürfnisse der Organisation und Teilnehmenden anhand von Bedarfsanalysen impliziert ist - wodurch persönliche Relevanz, Transfermotivation und Anwendungsmöglichkeit der Trainingsinhalte gefördert wird. Andererseits, weil transferförderliche Trainings durch den Einsatz fundierter Lehrmethoden unter Berücksichtigung individueller Teilnehmendeneigenschaften definiert sind, wodurch Lernen für alle Teilnehmenden maximiert werden sollte. Schließlich hat diese Stellschraube den höchsten implikativen Wert, da sie von Personalverantwortlichen direkt modifiziert werden kann, während transferförderliche Eigenschaften der Arbeitsumgebung und Teilnehmenden von den einzelnen Mitarbeitenden abhängen.

7 Die Anwendung der Funktion für individuelle Leistung wurde auch von anderen Autoren auf Transferleistung übertragen (Baldwin, Ford \& Blume, 2009; Holton et al., 2000; Kehr, Bles \& Rosenstiel, 1999; Noe \& Schmitt, 1986; Solga, 2011b), allerdings wurde dabei bisher keine strikte Differenzierung zwischen direkten und indirekten Faktoren vorgenommen. So wurden beispielsweise als Möglichkeitsvariablen nicht nur die tatsächliche Anwendungsgelegenheiten betrachtet, sondern auch unzählige begünstigende Rahmenbedingungen wie Offenheit für Änderungen in der Arbeitsgruppe, Feedbackkultur, Vorgesetzten- und Peereinstellung oder erwartete Transferkonsequenzen. Zudem wird in Holtons Modell (2005) der Faktor „Fähigkeit“ (bzw. „Ability“) nicht auf Kompetenz, sondern auf kognitive Fähigkeiten und transferförderliche Trainingsgestaltung oder Anwendungsgelegenheiten bezogen (siehe auch Abschnitt 3.2.2 ab Seite 74). 
Durch diese theoriegeleitete strikte Differenzierung und Priorisierung von direkten bzw. primären und eher indirekten bzw. sekundären Transferdeterminanten erfolgte eine Komplexitätsreduktion des Transfermodells bei gleichzeitig ganzheitlicher Betrachtung des Kerntransferprozesses. Damit soll ein zielgerichtetes und systematisches Transfermanagement ermöglicht werden. Dabei muss jedoch beachtet werden, dass die drei Funktionsvariablen als multiplikativ verknüpft gelten. So können höhere Ausprägungen eines Faktors niedrigere Ausprägungen der anderen zu einem gewissen Grad kompensieren (Iddekinge, Aguinis, Mackey \& DeOrtentiis, 2018). Wobei Kompetenz als Basisvoraussetzung und damit als wichtigste Transferdeterminante betrachtet wird: "Without learning, there is nothing to transfer" (Sørensen, 2017, S. 51). Der multiplikative Zusammenhang bedeutet auch, dass Transferleistung nur zustande kommt, solange ein Mindestmaß aller drei Faktoren gegeben ist. Entsprechend muss Transfermanagement nicht nur den Kompetenz, sondern auch die beiden anderen Determinanten der Transferleistung umfassend maximieren, um die „Kluft“ zwischen potentiellem Können und tatsächlichem Handeln zu überbrücken (North et al., 2018).

Was das „Objekt“ von Transfermanagement ist, sollte durch die beschriebenen Präzisierungen des Trainingstransferbegriffs und der Transferleistung deutlich geworden sein. Offen ist allerdings noch das dazugehörende Verständnis von „Management“. Nach dem sogenannten „Managementzyklus“ bezeichnet der Managementbegriff die umfassende Steuerung von Unternehmensprozessen, die in neun übergeordnete Funktionen unterteilt werden können: 1) Analyse, 2) Zielsetzung 3) Planung, 4) Entscheidung, 5) Organisation, 6) Delegation, 7) Koordination, 8) Mitarbeiterführung und 9) Kontrolle. Um in der schnelllebigen Wirtschaftswelt wettbewerbsfähig zu bleiben, ist entscheidend sämtliche Steuerungsfunktionen in integrierten Managementsystemen auf die kontinuierliche Anpassung und Verbesserung auszurichten, was im sogenannten „Qualitätsmanagementansatz“ zusammengefasst ist. Kern des Qualitätsmanagements sind Verfahrensgrundsätze und -weisen für kontinuierliche Verbesserungsprozesse, die auf dem sogenannten PDCA-Zyklus ${ }^{8}$ dessen Ursprünge auf Shewhart (1939) zurückgeführt werden, beruhen. So sollen Unternehmensprozesse durch einen fortlaufenden Kreislauf aus Planen, Durchführen, Überprüfen und Handeln gesteuert und ständig zielgerichtet optimiert werden. Während sich die Anfänge des Qualitätsmanagements auf die kontinuierliche Verbesserung der Endprodukte bezogen (Zollondz, 2014), hat sich das Qualitäts-

8 Dieser bereits von Shewhart in den späten 1930er Jahren erdachte und dann von Deming weiterentwickelte Zyklus sieht eine kontinuierliche Abfolge von „Plan-Do-Check-Act“ vor. Dieser Zyklus weist Parallelen zur Aktionsforschung nach Lewin (1948) auf, die für erfolgreiche Veränderungsprozesse einen ständigen Kreislauf und Nutzung der Wechselwirkungen von Diagnose und Intervention durch sogenannte „Aktions-Reflexions-Prozesse“ empfiehlt. 
management zu einem umfassenden und integralen Bestandteil der Unternehmensführung entwickelt, was sich auch in letzten Revision der ISO 9001 im Jahr 2015 widerspiegelt. So ist es inzwischen die Basis der Steuerung sämtlicher Unternehmensprozesse. Allerdings ist weitläufig das Personalwesen und insbesondere die Personalentwicklung aus dem Qualitätsmanagement von Unternehmen immer noch ausgeklammert, obwohl sich die Managementgrundsätze für die kontinuierliche Verbesserung der Kompetenzentwicklung mindestens so gut eignen wie für Produktionsprozesse ${ }^{9}$. So folgt professionelle Trainingsgestaltung mit Transfermanagement basierend auf dem Evaluationsmodell von Solga (2011a; siehe Seite 21) genau diesen Verfahrensgrundsätzen.

In Anlehnung an Qualitätsmanagementgrundsätze wird „systematisches Transfermanagement für Führungskräftetrainings“ in dieser Arbeit definiert als sämtliche Maßnahmen zur Planung, Konzeption, Durchführung, Überprüfung und Optimierung von Führungskräftetrainings, die die Entwicklung und Übertragung beruflicher Handlungskompetenz in den Arbeitsalltag zielgerichtet fördern. Grundlage dafür und damit auch für das vorliegende Forschungsprojekt ist die Evaluation von Trainings.

${ }^{9}$ Durch die Reform des Weiterbildungssystems für öffentlich geförderte Weiterbildungen im Jahr 2003 wurden Qualitätsmanagement-Systeme (nachfolgend QMS) auf Bildungsprozesse übertragen. Diese QMS sind Handlungsleitlinien zur Evaluation durch Dokumentation, Entwicklung und Verbesserung der Weiterbildungsqualität. Betrachtet man die für Weiterbildung öffentlich angebotenen QMS, ist ersichtlich, dass zwar alle auf unterschiedliche Weise die Umsetzung des PDCA-Zyklus zur Überprüfung der Qualität vorgeben. Allerdings stellen nur manche QMS auch konkrete Qualitätsanforderung an die Gestaltung von Weiterbildungsinterventionen und sind eher auf Zertifizierung von Weiterbildungsanbietenden als auf Qualitätsgenerierung oder fundierte Transfersicherung von Weiterbildungsmaßnahmen ausgerichtet (siehe auch BIBBAnalyse der Webmonitor-Umfrage 2017). Eine Übersicht und Bewertung der verschiedenen QMS für Weiterbildung wurde von Stiftung Warentest in nachfolgendem Link zusammengefasst: https://www.test.de/Qualitaetsmanagement-Transparenz-ist-nicht-in-Sicht-1531451-0/ 


\subsection{Kapitelzusammenfassung}

In diesem Kapitel wurde der Gegenstandsbereich zentraler Konzepte der vorliegenden Forschungsarbeit präzisiert und theoretisch eingebettet. Dies soll die Einordnung und Strukturierung der in den nachfolgenden Abschnitten zusammengefassten Forschungserkenntnisse erleichtern.

In der vorliegenden Arbeit geht es um die Evaluation und Förderung des Transfers von Führungskräftetrainings, der am meisten institutionalisierten und relevantesten Form von betrieblicher Weiterbildung. Diese sind definiert als formelle „off-the-job“ Weiterbildungsmaßnahmen zur systematischen Erweiterung von Führungskompetenzen. Sie finden in Abstand zum Arbeitsalltag statt, um Gruppen zwischen acht und vierzehn Führungskräften in geschütztem Rahmen in Erkenntnisgewinn, Erfahrungsaustausch und Ausprobieren neuer Verhaltensweisen durch Trainer zu unterstützen. Hierfür werden klassischerweise durch Vortrag, Diskussion, Übung, Feedback oder angeleitete Reflexion berufliche Handlungskompetenzen vermittelt. Dazu zählen Fach-, Methoden-, Sozial- und Selbstkompetenzen. Zur Vermittlung interaktionaler Sozialkompetenzen gilt die kombinierte Trainingsmethode „Behavior Modeling“ als fundierte Technik, die Vortrag, Rollenspiel, Gruppenarbeit und Videofeedback systematisch integriert.

Das Modell der Trainingswirksamkeit von Kirkpatrick $(1967,1998)$ ist bis heute das Einflussreichste. Es definiert diese als multidimensionales Konstrukt anhand von vier Dimensionen. Felfe und Franke (2014) haben diese multidimensionale Betrachtung auf die systematische und umfassende Evaluation von Führungskräftetrainings übertragen: Die Dimensionen „Reaktion“ und „Lernen“ beschreiben dabei unmittelbaren Trainingserfolg durch Zufriedenheits- und Nützlichkeitsbewertungen, die sich in der Transfermotivation widerspiegeln, sowie Zuwachs von Führungskompetenzen. Die Dimensionen „Verhalten“ und „Resultate“ erfassen langfristigen Transfererfolg durch Anwendung der trainierten Führungskompetenzen und der daraus resultierenden Effekte anhand von verbessertem Führungsverhalten, wodurch Leistung von Führungskräften und ihren Teams gesteigert wird.

Trainingstransfer wird demzufolge als entscheidendes Maß der Trainingswirksamkeit betrachtet und ist als erfolgreiche Übertragung und Anwendung der in Trainings (Lernfeld) erworben Kompetenzen auf den Arbeitskontext (Funktionsfeld) definiert. Entsprechend dem zentralen Basismodell prozessorientierter Transferforschung von Baldwin \& Ford (1988) impliziert die Transferdefinition eine effektive Übertragung auf Herausforderungen des Arbeitsalltags (Generalisierung) und kontinuierliche Anwendung (Aufrecht- 
erhaltung) von trainierten Kompetenzen. Neben der multidimensionalen Operationalisierung der Trainingswirksamkeit sollte daher auch die inhaltliche und zeitliche Distanz zwischen Training und Transferkriterien (naher versus weiter Transfer nach angemessener Transferphase) für aussagekräftige Trainingsevaluationen explizit berücksichtigt werden.

Die hier verwendete Definition von Trainingsevaluation umfasst formative und summative Qualitäts- und Erfolgskontrollen von Trainings und beinhaltet eine Konzeptions-, Durchführungs-, Wirksamkeits- und Wirtschaftlichkeitsbewertung. Nach Alkin (2004) lassen sich Evaluationsmodelle nach Bewertungs-, Methoden- und Anwendungsfokus differenzieren. Für eine umfassende Evaluation sollten jedoch alle drei Aspekte integriert werden. Die zentrale Theorie, des Bewertungsfokus stammt von Scriven (1991), aus der die grundlegende Unterscheidung von Evaluationsperspektiven in „formative“ und „summative" Evaluation hervorgeht. Das zentrale Modell des Methodenfokus stellt Kirkpatricks multidimensionale Operationalisierungsstruktur der Trainingswirksamkeit dar. Als Basismodell des Anwendungsfokus gilt das „CIPP-Modell“ von Stufflebeam (1983, 2004) mit den vier übergeordneten Evaluationsphasen Kontext- (C), Input- (I), Prozess$(P)$ und Produktevaluation $(P)$, die den Evaluationsstandards Nützlichkeit, Durchführbarkeit, Fairness und Genauigkeit entsprechen. Ein Evaluationsmodell, das Bewertungs-, Methoden- und Anwendungsfokus integriert und auf formale Personalentwicklung anwendet, stellt das Evaluationsmodell von Solga (2011a) dar. Die darin vorgegebenen summativen bzw. ergebnisorientierten Wirksamkeits- und Effizienzanalysen bewerten Nutzen und Wirtschaftlichkeit von Trainings. Formative bzw. prozessorientierte Ziel-, Umfeld-, Konzept-, Lehrprozess- und Wirksamkeitsanalysen können die dahinterliegenden Wirkmechanismen aufzeigen. Daher dient dieses Evaluationsmodell als konzeptioneller Rahmen des Forschungsprojektes. Im Rahmen dieser Arbeit werden davon die ergebnis- und prozessorientierte Wirksamkeitsanalyse sowie ihre theoretischen und praktischen Implikationen für zielgerichtete Transfermanagementempfehlungen beschrieben.

In Anlehnung an Qualitätsmanagementgrundsätze wird hier systematisches Transfermanagement durch zielgerichtete Maßnahmen zur Überprüfung und Förderung von Trainingstransfer definiert. Dafür muss Transfermanagement nicht nur Kompetenz, sondern auch Transfermotivation und -möglichkeit maximieren, um die „Kluft“ zwischen potenziellem Können und tatsächlichem Handeln aufzulösen. Die nachfolgend zusammengefassten Befunde legen nahe, dass sich Transfermanagementaktivitäten lohnen (siehe Abschnitt 3.1 ab Seite 31) und zeigen auf, welche Wirkmechanismen und Transferfaktoren dafür identifiziert wurden (siehe Abschnitt 3.2 ab Seite 43). 


\section{Zentrale Forschungsbefunde über Trainingswirksamkeit}

Seit Fleishman (1953) die unzureichende Wirksamkeit und das "Transferproblem“ von Trainings als zentrales Thema in die Trainingsforschung eingeführt hat, wurde intensiv an der Ergründung und Lösung des Transferproblems geforscht. In den ersten Jahrzehnten lag der Forschungsfokus auf der ergebnisorientierten Überprüfung der Wirksamkeit verschiedener Personalentwicklungsmaßnahmen auf organisationaler Makroebene. Dabei wurde der Frage nachgegangen ob Führungskräftetrainings generell wirksam sind und ob evidenzbasierte Trainingsgestaltung in erzieltem Trainings- und Transfererfolg resultiert. Die daraus entstandenen metaanalytischen Befunde zur Wirksamkeit von Führungskräfteentwicklungsmaßnahmen werden im ersten Abschnitt dieses Kapitels betrachtet. Daran anknüpfend wird der Forschungsstand ergebnisorientierter Trainingsforschung zusammengefasst und dessen Lücken aufgezeigt. Abgesehen von metaanalytischen Moderationsanalysen gibt die ergebnisorientierte Betrachtungsweise jedoch nur wenig Aufschluss über Handlungsimplikationen zur Lösung des Transferproblems.

Seit den 1980er Jahren nimmt daher die prozessorientierte Transferforschung das Feld der Trainingsforschung zunehmend ein, um der wesentlich komplexeren Frage nach den Wirkmechanismen im Trainings- und Transferprozess auf individueller Mikroebene nachzugehen. Welche Transferfaktoren durch die von Baldwin et al. (2017) als „Explosion“ beschriebene Forschungsdisziplin für die Lösung des Transferproblems als bestätigt gelten, welche Wirkmechanismen daraus bisher abgeleitet werden konnten und welche Forschungslücken nach wie vor bestehen ist im zweiten Abschnitt dieses Kapitels zusammengefasst.

Da das Transferproblem in einem doppelten Sinn nicht nur aufgrund von Forschungslücken weiterhin besteht, sondern auch aufgrund mangelnden Forschungstransfers in die Praxis, werden im dritten Abschnitt dieses Kapitels zudem das Research-Practice-Gap bezüglich fundierter Transfermanagementaktivitäten in der Trainingspraxis betrachtet und Ansätze zur Lösung zusammengefasst. 


\subsection{Ergebnisorientierte Trainingsforschung}

\subsubsection{Metaanalytisch bestätigte Befunde}

Die ergebnisorientierten Befunde zur Wirksamkeit formeller Führungskräfteentwicklung wurden bereits in vorliegenden Metaanalysen mehrfach systematisch zusammengefasst. Darin wurden vorwiegend Evaluationen von Führungskräftetrainings als zentrale Führungskräfteentwicklungsmaßnahme berücksichtigt. Diese systematisch integrierenden Betrachtungen der vorliegenden summativen Einzelbefunde, bieten eine belastbare Antwort auf die Frage, inwiefern Führungskräftetrainings wirksam sind und geben zudem durch Moderatoranalysen Anhaltspunkte, welche übergeordneten Faktoren die Wirksamkeit auf organisationaler (Makro-)Ebene beeinflussen. Daher werden für eine umfassende, aussagekräftige und übersichtliche Darstellung des ergebnisorientierten Forschungsstandes zur Wirksamkeit von Führungskräftetrainings nachfolgend die MetaanaIysen zur Effektivität formeller Führungskräfteentwicklung zusammengefasst.

Die Ausgangsbasis der Metaanalysen zur Effektivität formeller Führungskräfteentwicklung bildet die Metaanalyse von M. J. Burke und Day (1986). Aufgrund ihrer Datierung umfasste sie zwar nur Evaluationsstudien bis 1982, dennoch gilt sie als Paradebeispiel einer umfassenden Überprüfung der Wirksamkeit von Führungskräfteentwicklung und hat den Ansatz nachfolgender Metaanalysen dazu entscheidend geprägt. Sie betrachtete die Studien differenziert nach sechs Ausprägungen von Trainingsinhalten, sieben Ausprägungen von Trainingsmethoden und vier Ausprägungen von Erfolgskriterien (subjektiver und objektiver Lernerfolg, subjektive Verhaltensänderung und objektive Veränderung von Resultaten). Anhand der Analyse von insgesamt 70 Evaluationsstudien kamen die Autoren zu dem Schluss, dass Maßnahmen zur Führungskräfteentwicklung insgesamt nur moderate Effekte aufweisen, wobei die Wirksamkeit abhängig von Trainingsinhalten und -methoden ist bzw. deren Passung sehr unterschiedlich bewertet wurden. Zudem zeigte auch die Ausprägung der gewählten Erfolgskriterien einen Einfluss auf die Ergebnisse, wobei dies wiederum von dem jeweiligen Trainingsinhalt und der methode abhängig war. Aufgrund der hier gewählten Vorgehensweise in der Datenanalyse muss bei Ergebnisbetrachtungen beachtet werden, dass Evaluationsstudien mit mehreren abhängigen Variablen ein größeres Gewicht in den Ergebnisaussagen erhalten haben. Wie auch in nachfolgenden Metaanalysen wurde in dieser Metaanalyse der Trainingsbegriff im weiteren Sinne verstanden und umfasste sämtliche Führungskräfteentwicklungsmaßnahmen. Trotz vieler Fragen, die ihre Analyse aufwarfen, leiteten M. J. Burke und Day (1986) aus ihren Ergebnissen folgende zentrale Handlungsimplikationen $a b$ : 
1. Evaluationsforschende sollten die Dokumentation ihrer Studien verbessern insbesondere bezüglich Limitationen, Reliabilitäten von Kriteriums- und Prädiktorvariablen, Stichprobeneigenschaften und methodischem Vorgehen.

2. Die Kombination von unterschiedlichen Trainingsmethoden führt nicht automatisch zu höherem Trainings- bzw. Transfererfolg. Daher sollten sich Trainer und Entscheidungsträger aus Unternehmen nicht auf Beschreibungen von Trainingsinhalten verlassen, sondern bewusst Interventionen auswählen, deren methodischer Aufbau zu identifizierten Zielkriterien passt. Zudem sollten Evaluationen zur Qualitätssicherung verstärkt genutzt werden.

3. Die Trainererfahrung könnte ein signifikanter Einflussfaktor für die Effektivität von Führungskräftetrainings sein, der berücksichtigt werden sollte.

Die Autoren betrachteten die Studienbasis für konkretere Empfehlungen jedoch als unzureichend. Identifizierte Forschungslücken lagen insbesondere in einer geringen Zahl von Evaluationsstudien, die Zusammenhänge von Trainingsinhalt und -methode mit gewählten Evaluationskriterien betrachteten. Zudem wurde der zu selten berücksichtigte Einfluss von Trainererfahrung und -qualifikation in Evaluationsstudien bemängelt. Darüber hinaus kritisierten M. J. Burke und Day (1986) die lückenhafte Dokumentation bzw. unzureichende Information zum Evaluationsgegenstand und -vorgehen der vorliegenden Studien und forderten transparentere Darstellungen und vielfältigeren Methodeneinsatz in der Evaluationsforschung. Da Evaluationsstudien typischerweise auf Selbsteinschätzungen und Kurzzeitbetrachtungen basierten, sollten weitere Quellen und längere Betrachtungszeiträume ergänzt werden.

Collins und Holton (2004) kamen durch ihre metaanalytischen Untersuchungen auf eine deutlich bessere Bewertung der Trainingseffektivität als M. J. Burke und Day (1986), insbesondere beim Erfolgskriterium Wissensvermittlung. Die Autoren warnten jedoch aufgrund unterschiedlicher methodischer und konzeptueller Herangehensweisen vor einem direkten Vergleich mit der vorangegangenen Metaanalyse. Abgesehen von dem zeitlichen Faktor, könnte eine Erklärung dafür der Einbezug verschiedener organisationaler Hintergründe sein, da die Analyse von Burke und Day (1986) vorwiegend Evaluationsstudien aus der Wirtschaft beinhaltete. Collins und Holton (2004) schlossen zeitlich und inhaltlich an die Metaanalyse von M. J. Burke und Day (1986) an und betrachteten anhand von 83 Studien aus Wirtschaft, Bildung, Regierung, Medizin und Militär den nachfolgenden Zeitraum von 1982 und 2001. Dabei wurde ebenfalls der unterschiedliche Einfluss evaluierter Erfolgskriterien analysiert, allerdings wurde eine andere Kategorisierung verwendet (Wissen, Expertise und Leistung auf individueller, Gruppen- und Organisationsebene). Die Analysen zeigten auf, dass Führungskräfteentwicklungsmaßnah- 
men substanzielle Wissens- und Kompetenzentwicklungen bewirken können, wobei Bedarfsanalysen für eine Training-Trainee-Passung als entscheidend betrachtet wurden. Zusätzlich analysierten sie den Einfluss des gewählten Evaluationsdesigns. Die größten Erfolgseffekte resultierten aus Evaluationsstudien mit Eingruppen-Prä-Posttestplänen.

Eine weitere Metaanalyse zur Veränderbarkeit von führungsrelevanten Eigenschaften von Avolio, Reichard, Hannah, Walumbwa und Chan (2009) bestätigte ebenfalls die Effektivität von Führungskräfteentwicklungsmaßnahmen wobei die relevanten Effektstärkeberechnungen eine geringere Wirksamkeit als nach Collins und Holton (2004) nahelegen. Allerdings basierte dieser Befund auf nur 37 tatsächlichen Führungskräfteentwicklungsevaluationen von insgesamt 200 betrachteten Evaluationsstudien, da der Schwerpunkt auf genereller Beeinflussbarkeit von Führungseigenschaften lag (größtenteils gemessen an kurzfristigen Veränderungen durch Verhaltensmanipulation). Zudem waren die Ergebnisse zwar positiv bezüglich der Wirksamkeit von Führungskräfteentwicklung, aber bezüglich Moderatorwirkungen eher unspezifisch. Somit liefert diese Metaanalyse für die vorliegende Arbeit über diese Einordnung hinaus keinen weiteren Erkenntnisgewinn, weshalb sie nicht weiter ausgeführt wird.

Passendere Aussagekraft zum hier betrachteten Forschungsgegenstand weist dagegen die Metaanalyse von Taylor, Russ-Eft und Taylor (2009) auf, die gezielt Evaluationsstudien zum Transfererfolg von Führungskräfteentwicklungsmaßnahmen zusammenfasste. Ihre Metaanalyse basierte auf 107 Evaluationsstudien zur Transferüberprüfung von Führungskräfteentwicklungsmaßnahmen, die zwischen 1963 und 2003 veröffentlicht wurden. Sie analysierten den Zusammenhang von Transfererfolg und fünf Hauptmoderatoren: 1) Datenquelle (Selbst-, Mitarbeiter-, Kollegen- und Vorgesetzteneinschätzung), 2) Studienquelle (veröffentlicht vs. unveröffentlicht), 3) Organisationskontext (Wirtschaft, Militär und Sonstige), 4) Studiendesign und -qualität und 5) Trainingsinhalt. Die Analysen getrennt nach Datenquelle zeigten, dass Studien mit Selbsteinschätzungen die größten und mit Mitarbeitendeneinschätzungen die geringsten Effektstärken hervorbrachten, wobei Selbst- und Vorgesetzteneinschätzungen die robustesten Ergebnisse aufwiesen. Dieses Ergebnismuster zeigte sich insbesondere bei Trainings zur Vermittlung interaktionaler Kompetenzen, sowohl bei publizierten als auch unveröffentlichte Studien und war unabhängig vom jeweiligen Studiendesign. Bei Betrachtung nichtmilitärischer Organisationen zeigte sich für folgende Moderatorausprägungen ein besonders großer Transfereffekt: 1) Bewertende wissen über Gruppenzuteilung bzw. Trainingsteilnahme Bescheid, 2) Transferkriterien sind von Trainingsinhalten abgeleitet, 3) Trainings basieren auf Bedarfsanalysen und bieten Übungsgelegenheiten. Die Metaanalyse zeigte darüber hinaus, dass Vorgesetztenbefragungen, die mit Abstand am häufigsten eingesetzte Datenquelle zur Transferüberprüfung ist. Zumindest für diesen Forschungsausschnitt bedeutet 
dies, dass die Anregung von M. J. Burke und Day (1986) bezüglich Nutzung anderer Datenquellen umgesetzt wurde.

Die kurz darauf veröffentlichte Metaanalyse von Powell und Yalcin (2010) schloss an die Arbeit von Collins und Holton (2004) an, um die deutlich größeren Effektstärken im Vergleich zu M. J. Burke und Day (1986) zu ergründen. Sie setzte jedoch einen engeren Fokus in Bezug auf das Organisationssetting und berücksichtigte nur Evaluationen aus der Wirtschaft. Ihre Ergebnisse basierten daher auf nur 62 Studien, obwohl sie einen relativ weiten Beobachtungszeitraum von 1952 bis 2002 erfasste. Schwerpunkt der Analysen war die Überprüfung, ob sich der Wirkungsgrad von Führungskräftetrainings weiterentwickelt hat. Jedoch wurden über 50 Jahre hinweg gleichbleibende mittlere Effekte evaluierter Trainings gefunden. Darüber hinaus wurden die Daten zusätzlich getrennt nach den Evaluationskriterien basierend auf Kirkpatricks Evaluationsmodell (1967, 1994) analysiert (wobei die Reaktionsebene nicht berücksichtigt wurde) sowie Studiendesign und Hierarchielevel. Entgegen der Annahme waren die Transfereffekte unabhängig vom Evaluationsdesign. Allerdings zeigte sich eine Interaktion von Studiendesign und Kriteriumsebene dahingehend, dass die Lerneffekte bei Kontrollgruppendesigns ohne Vorerhebung und Eingruppen-Prä-Posttestdesigns größere Effekte zeigten als in Kontrollgruppendesigns mit Vorerhebung. Zudem wiesen Kriterien auf der Lernebene deutlich größere Effektstärken auf als Kriterien der Transfer- und Resultatebene.

Die aktuellste und umfangreichste Metaanalyse stammt von Lacerenza et al. (2017). Sie knüpften an die weit verbreiteten Zweifel an der Effektivität von Führungskräftetrainings an (siehe auch J. Schwartz et al., 2014), kamen aber zu einem weitaus positiveren Ergebnis als die vorangegangenen Metaanalysen. Die Autoren betrachteten 335 Primärstudien aus dem Wirtschaftskontext, die zwischen 1882 und 2014 veröffentlicht wurden. Im Gegensatz zu den vorangegangenen Metaanalysen legten sie den Analysefokus auf mögliche Wirkfaktoren für Trainings- bzw. Transfererfolg (ebenfalls getrennt nach Kirkpatricks Evaluationskriterien). Darüber hinaus untersuchten sie 15 Moderatoren der Trainingskonzeption, -durchführung und Evaluation, um konkrete und umfassend fundierte Handlungsempfehlungen für effektive Gestaltung von Trainings und deren Evaluation ableiten zu können. Sie zeigten, dass Führungskräftetrainings substanzielle Effektstärken für die Reaktions- $(\delta=.63)$, Lern- $(\delta=.73)$, Transfer- $(\delta=.82)$ und Resultatebene ( $\delta$ $=.72$ ) hervorbrachten. Führungskompetenzen sind demnach nachweislich trainierbar.

Da die Analysen zudem zeigten, dass formelle Trainings selbstgesteuerter informeller Führungskräfteentwicklung bezüglich Effektivität überlegen sind, sollte die Bedeutung formeller Trainingsmaßnahmen nicht unterschätzt werden. Die Maßnahmenwirksamkeit hängte jedoch entscheidend von evidenzbasierter und praktisch relevanter Konzeption 
und Durchführung ab. Daraus wurden folgende zentrale Transferfaktoren für Führungskräfteentwicklungsmaßnahmen abgeleitet:

- Einsatz formeller Führungskräfteentwicklungsmaßnahmen durch Trainings statt informeller Führungskräfteentwicklung

- Nutzung von Bedarfsanalysen als Basis für Trainingsziele

- Einsatz von Feedback (wobei $360^{\circ}$-Ansätze keine größere Wirkung als einfaches Feedback zeigen) und didaktischem Methodenmix (insbesondere mit Übungsmöglichkeiten) in der Trainingsdurchführung

- Zeitliche Verteilung der Trainingsmaßnahmen auf mehrere Sitzungen

- Durchführung von Präsenztrainings (statt virtuelle Trainingsformen)

- Qualitätssicherung durch prozessbegleitende Evaluation der Trainingsgestaltung in Bezug auf die gewünschten Ziele

Zudem wurden Hinweise darauf gefunden, dass Trainingsinhalt, Freiwilligkeit der Teilnahme und Trainingsdauer die Maßnahmeneffektivität in Abhängigkeit der Evaluationsebene (Lernen, Transfer vs. Resultate) beeinflussen.

Insgesamt zeigen die Metaanalysen zur Effektivität von Führungskräfteentwicklung ${ }^{10}$, dass Führungskräftetrainings in der Wirtschaft durchaus positive Effekte erzielen können, wobei die Effektgröße über die Metaanalysen hinweg sehr unterschiedlich ausfallen. Worin sich die Analysen jedoch weitestgehend einig sind, ist die Tatsache, dass die Ergebnisse insbesondere von folgenden Aspekten abhängen: Trainingsinhalte (Vermittlung von Sozial-, Selbst- und Managementkompetenzen), Trainingsmethoden (Vortrag, Diskussion, Verhaltensmodellierung, Feedback, didaktischer Methodenmix), Trainingseigenschaften (Freiwilligkeit der Teilnahme, Nutzung einer Bedarfsanalyse, zeitliche Verteilung), erfasste Erfolgskriterien (Reaktion, Lernen, Verhalten bzw. Transfer und Resultate), betrachtete Transferzeit, Trainer- bzw. Evaluationsteam, Forschungsdesign (Kontroll- oder Eingruppenplan mit Post- oder Prä-Posttest), Studienund Organisationssetting, Datenquelle (Selbst-, Mitarbeiter-, Kollegen- oder Vorgesetzteneinschätzung) und Hierarchieebene der Teilnehmenden.

Eine Zusammenfassung der Metaanalysen zur Wirksamkeit formeller Führungskräfteentwicklung und ihrer zentralen Befunde enthält Tabelle 1 (ab Seite 37). Daraus ist er-

${ }^{10}$ Es gibt darüber hinaus unveröffentlichte Metaanalysen zur Effektivität von Führungskräfteentwicklung (Lai, 1996; Zhang, 1999), die jedoch kaum Beachtung in der Trainingsforschung erhalten haben. Sie bringen allerdings auch - soweit aus verfügbaren Zusammenfassungen und vorhandenen Zitationen ersichtlich (z. B. Collins \& Holton, 2004) - keine neuen Erkenntnisse mit sich. Daher werden sie in der vorliegenden Arbeit nicht weiter betrachtet. 
sichtlich, dass sich die Evaluationsforschung im Trainingsbereich in den ersten Jahrzehnten der Transferforschung vorwiegend mit der summativen Frage des Wirkungsgrades beschäftigt hat. In neueren Betrachtungen wird zunehmend der sogenannten Black Box des Trainingstransfers durch die Integration formativer Analysen Beachtung geschenkt. Insbesondere die aktuellste und umfangreichste Metaanalyse von Lacerenza et al. (2017) legt durch Nutzung zahlreicher Moderatoren bezüglich Eigenschaften der Intervention und Evaluation einen Fokus auf die metaanalytische Identifikation evidenzbasierter Transferfaktoren. Transferrelevante Teilnehmendeneigenschaften und Faktoren der Arbeitsumgebung sind jedoch nicht berücksichtigt. Daher muss beachtet werden, dass durch diese Moderatoranalysen noch keine Aussagen über dahinterliegende Wirkzusammenhänge der Transferfaktoren des Transferprozesses auf individueller Mikroebene möglich sind. 


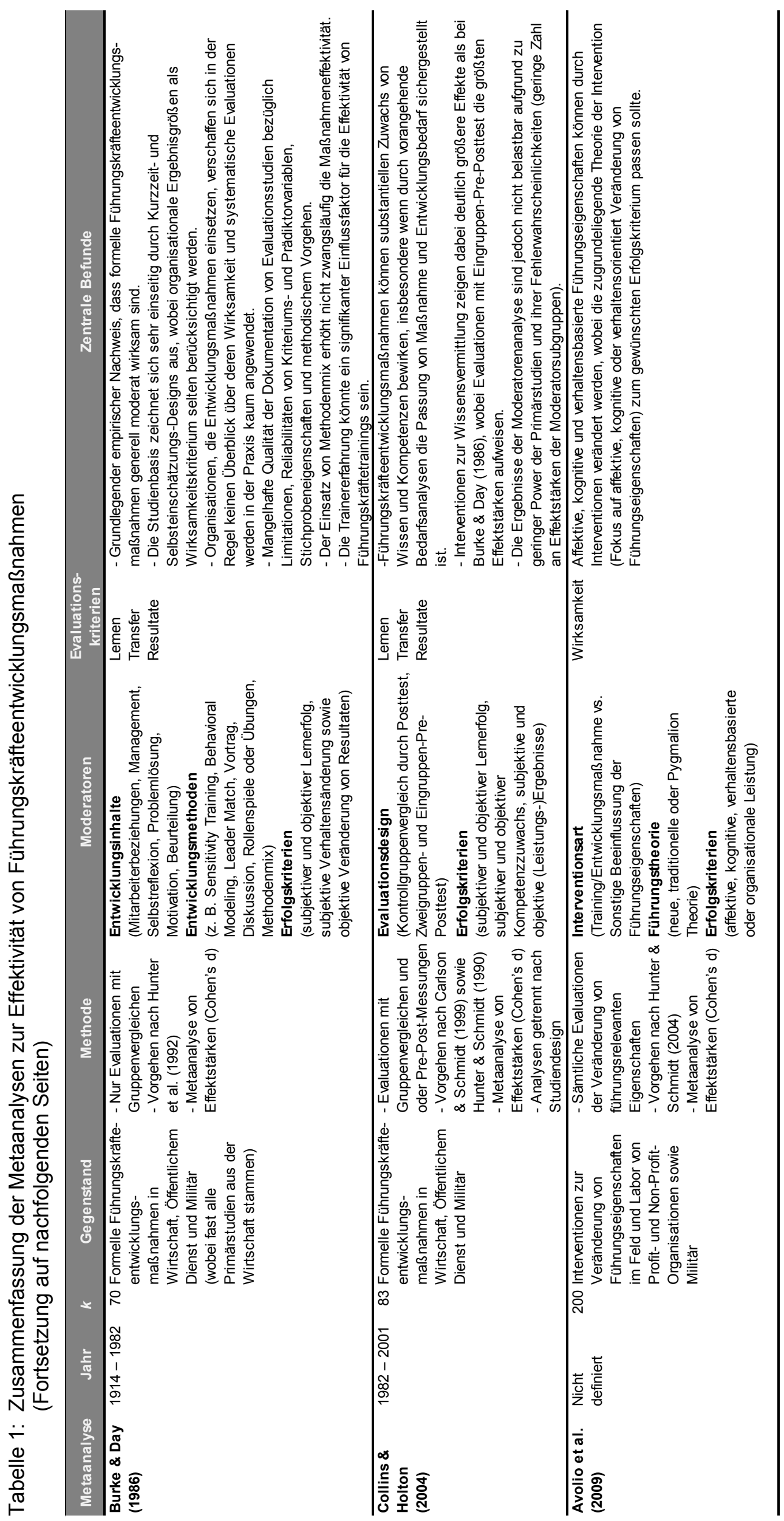




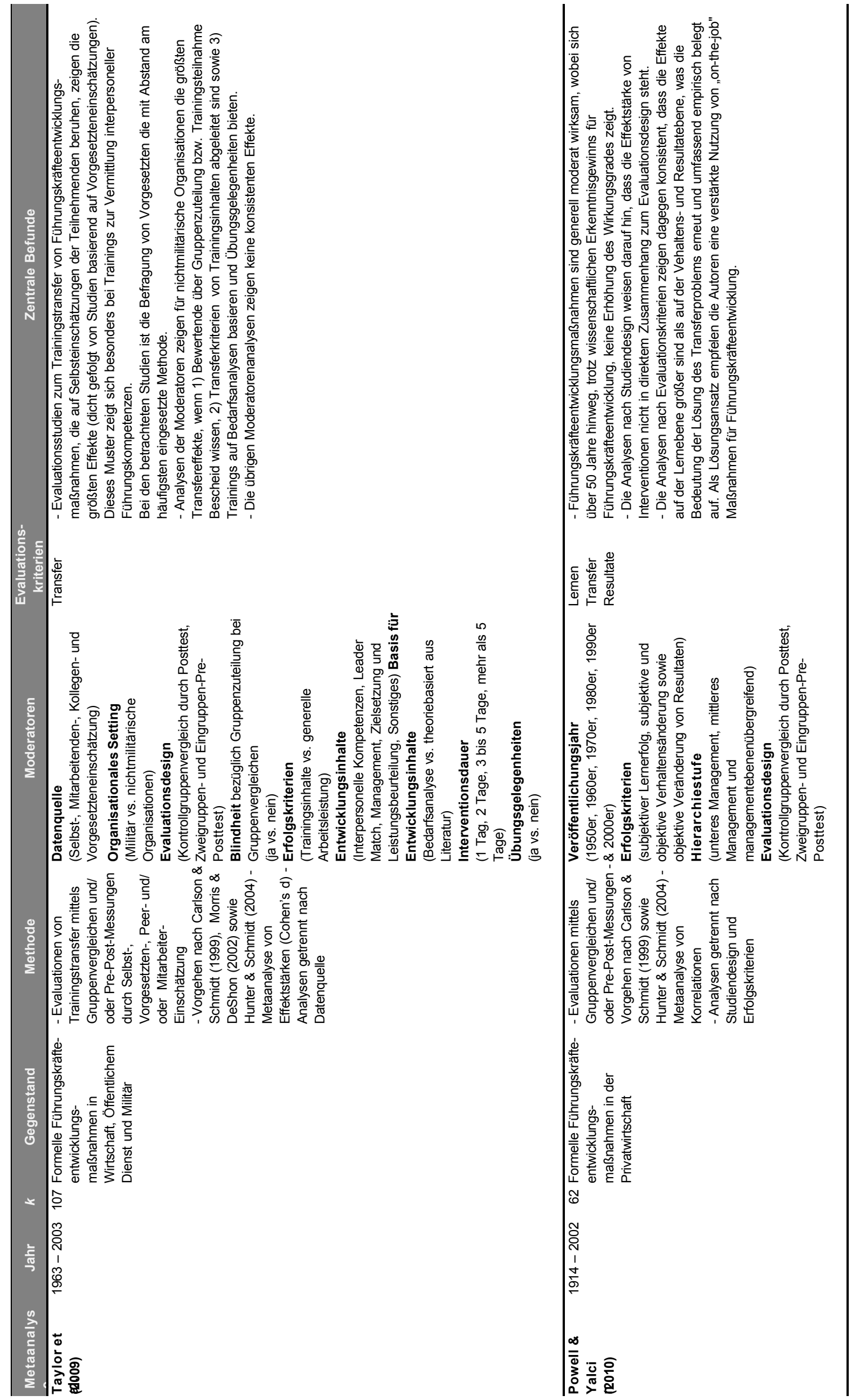




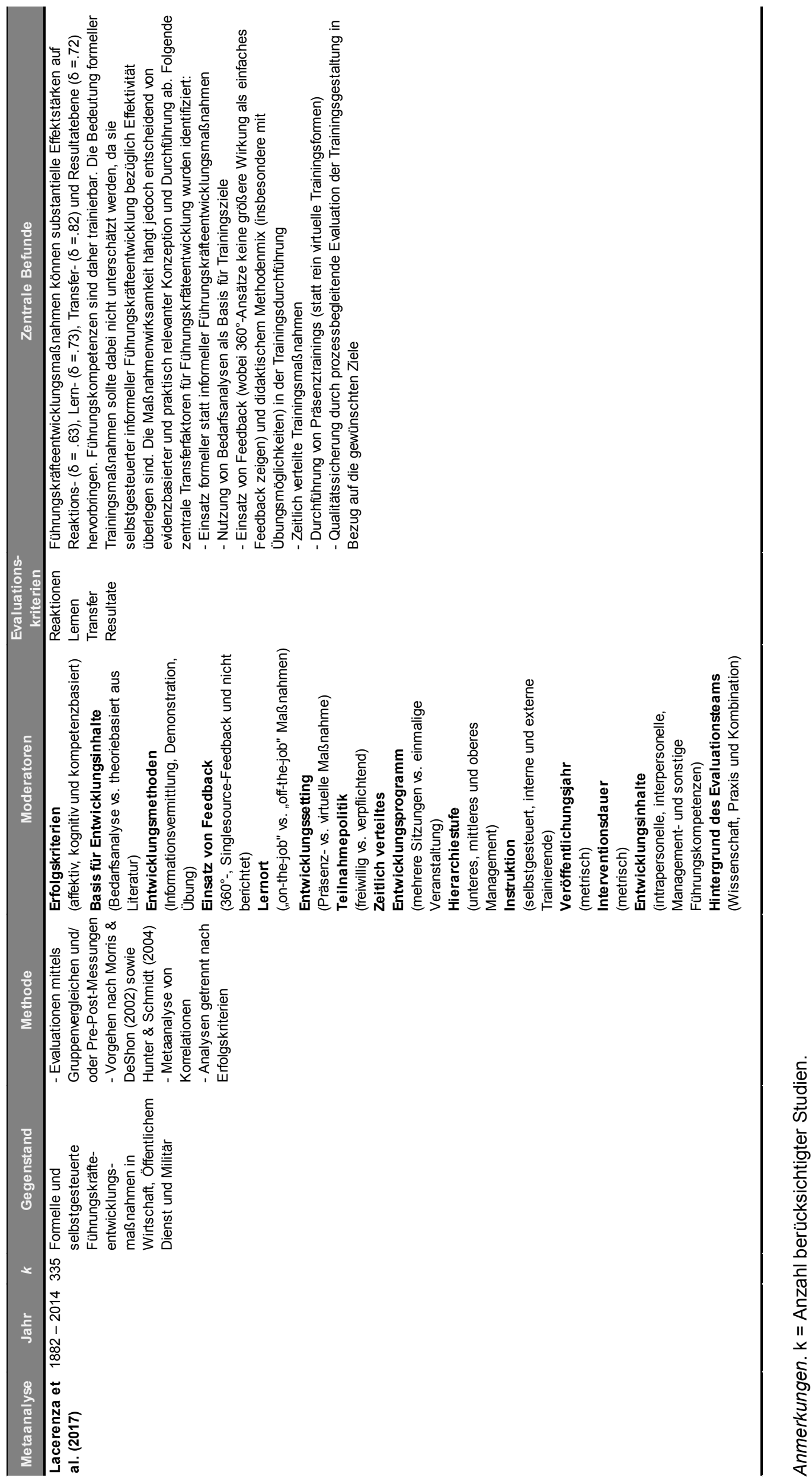




\subsubsection{Eigenschaften von Evaluationsstudien}

Für eine systematische Überprüfung, was vorliegende Evaluationsstudien von Führungskräfteentwicklung auszeichnet und welche Lücken dabei offenbleiben, werden nachfolgend die Eigenschaften der von Lacerenza et al. (2017) als relevante identifizierten 335 Evaluationsstudien zu Führungskräfteentwicklungsmaßnahmen näher beleuchtet $^{11}$.

Obwohl das einflussreichste Modell ergebnisorientierter Trainingsforschung von Kirkpatrick $(1967,1998)$ eine multidimensionale Erfassung der Trainingswirksamkeit anhand von vier Erfolgskriterien empfohlen hat, erfassten ca. 75\% der Studien lediglich eine Dimension der Trainingswirksamkeit und weitere 23\% erfassten zwei Dimensionen (meist Verhalten und Resultate). Nur 8 Studien operationalisierten drei Dimensionen, während keine Studie alle vier Dimensionen überprüfte. So können basierend auf bisherigen Führungskräftetrainingsevaluationen nur begrenzt Aussagen zu den im Modell angenommenen und häufig in Frage gestellten Zusammenhängen zwischen den Wirksamkeitskriterien gemacht werden. Betrachtet man die Häufigkeit der jeweiligen Kriterien zeigt sich, dass die Reaktion nur bei sieben der 335 Studien überprüft wurde. Lernen $(k=155)$ und Transferverhalten $(k=187)$ wurden dagegen am häufigsten evaluiert. Trotz ihrer erheblichen Konfundierungsprobleme wurden Resultate von 78 Evaluationsstudien erfasst. Diese Verteilung spiegelt jedoch nicht die Evaluationsaktivitäten der Trainingspraxis wider. Dort werden Interventionen vorwiegend auf der Reaktionsebene anhand von Nützlichkeits- und insbesondere Zufriedenheitseinschätzungen mittels sogenannter „Happy Sheets" evaluiert. Wesentlich seltener erfolgt eine Überprüfung des Lernerfolgs und nur vereinzelt werden Transferverhalten oder organisationale Ergebnisgrößen erfasst (Buren \& Erskine, 2002; Kaiser \& Curphy, 2013).

Ein weiterer Gegensatz zum empfohlenen Vorgehen der Evaluationsforschung besteht in der Dokumentationsqualität evaluierter Interventionen. Obwohl bereits M. J. Burke und Day (1986) die unzureichende Beschreibung evaluierter Interventionen thematisiert haben, werden auch in den 30 Jahre später zusammengefassten Evaluationsstudien als relevant identifizierte Trainingseigenschaften nur teilweise berichtet. Beispielsweise machte jeweils ca. ein Drittel der Studien keine Angaben zu eingesetzten Trainingsmethoden oder Interventionsdauer und nur 65 Studien gaben an, ob das evaluierte Training auf einer Bedarfsanalyse basierte. Selbst Basisinformationen wie konkrete Trainingsinhalte oder genaue Messzeitpunkte der Evaluation wurden nicht immer angegeben.

${ }^{11}$ Eine Tabelle der Einzelstudien und ihrer zentralen Ausprägungen ist aufgrund des Umfangs hier nicht übersichtlich abbildbar, kann jedoch bei der Autorin angefordert werden. 
Neben der Operationalisierung und Dokumentation zeigt sich auch bezüglich eingesetzter Studiendesigns eine Kluft zwischen methodischem Ideal und der Evaluationspraxis. Als Königsweg der Evaluationsforschung wird das Solomon-Vier-Gruppen-Design empfohlen (Martin et al., 2017; Rosenstiel et al., 2005), das Messwiederholung und Kontrollgruppendesign verknüpft, wobei auch Testeffekte durch Mehrfachmessung ohne Intervention identifizieren kann (Solomon, 1949) ${ }^{12}$. Dennoch ist das statistisch schwächste Design durch Eingruppen-Posttests in der Trainingsforschung keine Seltenheit. In der Metaanalyse von Lacerenza et al. (2017) wurden diese Primärstudien ausgeschlossen, da sie für summative Betrachtungen keine belastbare Aussage liefern können. Allerdings nutzen auch von den bezüglich Evaluationsdesign vorselektierten 335 Studien nur 36\% einen Vergleich unabhängiger Stichproben und nur 17\% verwendeten eine Kombination aus Messwiederholung und unabhängigen Stichproben. Die überwiegende Mehrheit systematisch evaluierter Führungskräftetrainingsevaluationen wählte Eingruppen-PräPosttest-Designs. Hinzu kommt der Aspekt, dass vorliegende Evaluationsdesigns vorwiegend reine Selbsteinschätzungen per Fragebogen mit geringem zeitlichem Abstand zur Intervention verwendeten. In Kombination mit der rudimentären Beschreibung der dabei bewerteten Interventionen lassen diese summativen Einzelbefunde nur wenig Kausalschlüsse oder praktische Anhaltspunkte zu, wie Trainings gestaltet sein müssen, um ihre nachhaltige Wirksamkeit zu fördern.

\subsubsection{Zusammenfassung ergebnisorientierter Trainingsforschung}

Obwohl Forschung und Praxis die nachhaltige Trainingswirksamkeit insbesondere formeller Softskill-Trainings in Frage stellen (Kaiser \& Curphy, 2013; Laker \& Powell, 2011; Poell, 2017; J. Schwartz et al., 2014; Sørensen, 2017), legen die zusammengefassten metaanalytischen Befunde nahe, dass zumindest systematisch evaluierte Führungskräftetrainings substantielle Wirksamkeit auf allen vier Dimensionen hervorbringen können. Der Wirkungsgrad ist jedoch maßgeblich von Interventions- und Evaluationseigenschaften abhängig. Denn "[...] the effectiveness of managerial leadership development programs varied widely; some programs were tremendously effective, and others failed miserably" (Collins \& Holton, 2004, S. 232).

Auch wenn die ergebnisorientierte Trainingsforschung aufzeigt, dass Führungskräfteentwicklungsmaßnahmen nachhaltige Wirksamkeit zeigen können, darf nicht übersehen werden, dass die Ergebnisse nicht repräsentativ für die Grundgesamtheit von Führungs-

12 Ein Anwendungsbeispiel stellt die Evaluation von Holdnak, Clemons und Bushardt (1990) dar: Gruppe1: Prätest-Intervention-Posttest; Gruppe2: Prätest-Posttest; Gruppe3: InterventionPosttest; Gruppe4: Posttest. 
kräfteentwicklungsmaßnahmen sind, sondern lediglich für die Subgruppe fundiert evaluierter Maßnahmen, wobei diese in der Personalentwicklungspraxis eher zur Ausnahme gehören (Wang \& Wilcox, 2014; Westermann \& Siemer, 2009). Dadurch kann auch die vermeintlich widersprüchliche Kritik bezüglich unzureichender Trainingswirksamkeit aus Praxis und Forschung erklärt werden. Denn es kann aus verschiedenen Gründen angenommen werden, dass Maßnahmen, die evaluiert werden, mit höherer Wahrscheinlichkeit auch effektiver sind (siehe auch Saks \& Burke, 2012). Beispielsweise, weil die Durchführung von Evaluationen an sich transferförderlich wirken kann, da evaluierte Maßnahmen auf Teilnehmende bedeutender wirken und die Überprüfung von Kompetenzentwicklung und Transfererfolg gleichzeitig auch eine Wiederauffrischungsmaßnahme der Trainingsinhalte darstellen können. Evaluationen haben zudem häufig eine Optimierungsfunktion für Folgemaßnahmen. Daher haben Organisationen, die regelmäßig Evaluationen ihrer Personalentwicklungsmaßnahmen durchführen, mit hoher Wahrscheinlichkeit bereits Optimierungsschlaufen durchlaufen. Wobei insbesondere wissenschaftlich fundierte Evaluationen die Qualität von Evaluationsergebnissen und damit auch die Optimierungsfunktion sowie erfolgreiche Anwendbarkeit von Erkenntnissen erhöhen (Westermann \& Siemer, 2009). Weiter kann angenommen werden, dass Organisationen, die sich die Mühe einer Evaluation machen, generell einen Fokus auf fundierte Durchführung von Personalentwicklungsmaßnahmen setzten.

Die Betrachtung relevanter Einzelbefunde zeigt zudem immer noch Forschungslücken ergebnisorientierter Trainingsforschung für eine belastbarere Aussagekraft auf, insbesondere bezüglich Studien, die Trainingswirksamkeit auf allen vier Dimensionen nach Kirkpatrick betrachten. Darüber hinaus sollten Studiendesigns gewählt werden, die eine Kombination aus Kontrollgruppenvergleichen und Mehrfachmessung mit angemessenem zeitlichem Abstand zum Training sowie mit der Nutzung weiterer Datenquellen in Ergänzung zur reinen Selbsteinschätzung (insbesondere für die Resultatebene) aufweisen. Auch einer aussagekräftigeren Dokumentationsqualität relevanter Eigenschaften von Intervention und Evaluation sollte mehr Beachtung geschenkt werden. Zudem ist eine rein ergebnisorientierte Betrachtung für die Lösung des Transferproblems unzureichend, das Ausmaß der Transferlücke aufzeigt. Offen bleibt jedoch die Frage, durch welche Faktoren und Maßnahmen Transfer gefördert werden kann. Dieser Frage geht die prozessorientierte Transferforschung nach, die durch die Ergründung der Wirkmechanismen im Trainings- und Transferprozess auf individueller (Mikro-)Ebene die ergebnisorientierte Trainingsforschung zunehmend ablöst. Welche Transferfaktoren für die Lösung des Transferproblems daraus als bestätigt gelten, welche Wirkmechanismen daraus bisher abgeleitet werden konnten und welche Forschungslücken nach wie vor bestehen ist im folgenden Abschnitt zusammengefasst. 


\subsection{Prozessorientierte Trainingsforschung}

Als Ausgangspunkt der seit den 1980ern zunehmend explodierten prozessorientierten Transferforschung gilt das erste umfassende Review dieses Forschungsstranges, in dem Baldwin und Ford (1988) vorliegende Definitionen und Befunde zu Transferdeterminanten in einem integrativen Transferprozessmodell zusammengefasst haben (siehe auch Abschnitt 3.2.2 ab Seite 72). Demnach hängt der Transfererfolg von drei übergeordneten Trainingsinputfaktoren ab: Teilnehmendenmerkmale (Fähigkeit, Persönlichkeit und Motivation), Trainingsgestaltung (Inhalt, Lernprinzipien und zeitliche Verteilung) sowie Arbeitsumgebung (Unterstützung und Anwendungsmöglichkeit). Alle drei Inputfaktoren wirken vermittelt über den Trainingsoutput in Form von Lernerfolg und Wissensspeicherung auf den Transfer. Darüber hinaus wird ein direkter Einfluss von Faktoren der Teilnehmendenmerkmale und Arbeitsumgebung auf die Generalisierung und langfristige Aufrechterhaltung neu erlernter Kompetenzen im Arbeitsalltag angenommen.

Durch die zahlreichen Befunde der darauffolgenden Jahrzehnte können diese übergeordneten Zusammenhänge als bestätigt gelten. Zudem wurden die drei übergeordneten Trainingsinputfaktoren durch konkrete Transferdeterminanten immer weiter spezifiziert und überprüft. Die umfassenden Forschungsaktivitäten wurden seitdem vielfach durch Reviews (Baldwin et al., 2017; L. A. Burke \& Hutchins, 2007, 2008; Cheng \& Hampson, 2008; Cheng \& Ho, 2001; Ford et al., 2018; Gegenfurtner, Veermans, Festner \& Gruber, 2009; Hochholdinger et al., 2008; Holton, 2005; Salas et al., 2012; Tonhäuser \& Bücker, 2016) und Metaanalysen (Bauer et al., 2016; Blume et al., 2010; Colquitt, LePine \& Noe, 2000; Gegenfurtner, 2011; Gegenfurtner, Könings, Kosmajac \& Gebhardt, 2016; Gegenfurtner, Veermans \& Vauras, 2013; Huang, Blume, Ford \& Baldwin, 2015; Hughes, Zajac, Woods \& Salas, 2019; S. D. Hutchins, Wickens, Carolan \& Cumming, 2013; Lacerenza et al., 2017; Reinhold et al., 2018) zusammengefasst. Während die Metaanalyse von Blume et al. (2010) alle Transferfaktoren und ihre einzelnen Zusammenhänge mit Trainings- und Transfererfolg betrachtete, beschäftigten sich die übrigen Metaanalysen mit vordefinierten Ausschnitten des Transferprozesses und den Wirkzusammenhängen zentraler Transferdeterminanten. Dabei wurde insbesondere die Transfermotivation als zentraler Transferprädiktor und dessen Mediationsfunktion zwischen Organisations-, Vorgesetzten- und Kollegenunterstützung näher analysiert.

Nachfolgend werden die Befunde über relevante Transferdeterminanten anhand der führenden Unterteilung nach Baldwin \& Ford (1988) in transferförderliche Teilnehmendenmerkmale, Trainingsgestaltung und Arbeitsumgebung zusammengefasst. Innerhalb dieser übergeordneten Kategorien werden zunächst die metaanalytisch bestätigten Transferdeterminanten aufgezeigt. Da darin vorwiegend Befunde bis 2014 berücksichtigt sind, 
wird in einem zweiten Schritt ein Überblick über Transferfaktoren gegeben, die in neueren Einzelbefunden von 2014 bis 2019 identifiziert wurden. Dabei wird als weiteres Ordnungskriterium innerhalb der drei Trainingsinputfaktoren die Unterteilung in Vorhersagekraft und Prädiktoren der jeweiligen Transferdeterminanten gewählt, um die bisher gefundenen Wirkzusammenhänge und Annahmen zur zeitlichen Wirkfolge zu verdeutlichen. Für die systematische Erfassung aller aktuellen und relevanten Befunde prozessorientierter Transferforschung wurde folgendes Vorgehen gewählt: 1) Um Befunde aller Disziplinen zu berücksichtigen, wurden alle EBSCOhost Datenquellen durchsucht, 2) als Suchbegriffe wurden „transfer of training“ oder "transfer of learning” gewählt, 3) berücksichtigt wurden englisch- und deutschsprachige nach Peer-Review-Prozess veröffentlichte Studien von 2014 bis einschließlich 2019, 4) davon wurden nur Feldstudien aus typischen Arbeitskontexten ausgewählt, um eine möglichst aussagekräftige Basis für den hier betrachteten Forschungsgegenstand zu erhalten. Studien aus (Hoch-)Schulkontexten, Militär und Gesundheitswesen, sowie Studien mit unzureichender Information bezüglich Kontext, Operationalisierung und Ergebnissen, wurden ausgeschlossen. Durch dieses Vorgehen wurden 61 relevante Studien identifiziert, die ergänzend zu den metaanalytischen Erkenntnissen zusammengefasst werden. Da die meisten Studien prozessorientierter Transferforschung nicht spezifisch auf Führungskräftetrainings ausgerichtet sind und es zu wenige Studien zu Führungskräftetrainings für belastbare Aussagen gibt, beziehen sich die nachfolgend zusammengefassten Befunde generell auf Trainingstransfer sämtlicher Personalentwicklungsmaßnahmen (sofern nicht explizit anders angegeben).

Nach dieser Zusammenfassung der Befunde zu einzelnen Wirkzusammenhängen, werden die einflussreichsten integrativen Transfermodelle vorgestellt, die versuchen den Transferprozess durch einen konzeptionellen Rahmen ganzheitlich zu erklären. Abschließend wird der Forschungsstand prozessorientierter Trainingsforschung zusammengefasst und aktuelle Befunde in die etablierten Transfermodelle integriert. 


\subsubsection{Befunde zu Determinanten des Trainingstransfers}

\section{Transferförderliche Teilnehmendenmerkmale}

\section{Metaanalytisch bestätigte Transferdeterminanten}

Kognitive Fähigkeiten zeigten generell den höchsten Zusammenhang mit Transfer im Vergleich zu allen anderen Transferdeterminanten (Blume et al., 2010; Colquitt et al., 2000; Huang et al., 2015). Betrachtet man die Befunde jedoch genauer zeigte sich dieser positive Zusammenhang nur bei maximaler (vs. typischer) Transferleistung und wurde durch Kompetenz mediiert (Huang et al., 2015). Zudem zeigte sich der Zusammenhang nur bei Trainings zur Vermittlung von "Closed Skills“13, jedoch nicht bei interaktionalen Führungskräftetrainings (Blume et al., 2010). Weitere stabile Persönlichkeitseigenschaften, die einen bedeutsamen Zusammenhang mit Transfer aufzeigten, sind Gewissenhaftigkeit und etwas schwächer auch Emotionale Stabilität (Blume et al., 2010; Colquitt et al., 2000; Huang et al., 2015). Allerdings zeigt hier ein genauerer Blick, dass Gewissenhaftigkeit nur einen Einfluss auf typische (vs. maximale) Transferleistung aufwies (Huang et al., 2015).

Veränderbare Teilnehmendeneigenschaften, die Transferleistung bedingen, werden oft auch als Trainingsoutcomes untersucht, um deren Determinanten zu ergründen und den Trainings- und Transferprozess tiefergehend zu beschreiben. In diesem Kontext wurden insbesondere verschiedene motivationale Aspekte untersucht, da motivationale Prozesse letztlich darüber entscheiden, ob erlernte Kompetenzen angewendet werden (Bandura, 1986). Neben Lern-, Trainings- und Transfermotivation ${ }^{14}$ (Bauer et al., 2016; Blume et al., 2010; Colquitt et al., 2000; Huang et al., 2015; Hughes et al., 2019; Reinhold et al., 2018), wurde auch die Transferwirkung volitionaler Konstrukte wie Selbstwirksamkeit ${ }^{15}$, internale Kontrollüberzeugung bzw. Leistungs- und Ergebniser-

${ }^{13}$ Blume et al. (2010) unterscheiden Trainings von "Closed Skills" (= Kompetenzen, die auch im Arbeitskontext auf dem gleichen Anspruchsniveau und auf die gleiche Weise wie im Trainingskontext direkt angewendet werden können) und "Open-Skills“ (= Kompetenzen, die im Trainingskontext eher als Prinzipien oder Grundlagen vermittelt werden, aber im Arbeitskontext auf die Lösung von meist komplexeren Herausforderungen übertragen werden müssen).

${ }^{14}$ Die unterschiedenen Motivationsarten bestimmen Richtung, Ausmaß und Ausdauer für generelle Lernbemühungen (Lernmotivation), für Teilnahme und Lernen im einem spezifischen Trainingskontext (Trainingsmotivation) bzw. für die Anwendung erlernter Kompetenzen (Transfermotivation).

15 Pretraining-Selbstwirksamkeit scheint allerdings bei interaktionalen Führungskräftetrainings weniger relevant zu sein als bei sonstigen „Open-Skills“ Trainings. Zudem zeigt nur bei interaktionalen Führungskräftetrainings die Posttraining-Selbstwirksamkeit einen etwas größeren Zusammenhang mit Transfer als die Pretraining-Selbstwirksamkeit (Blume et al., 2010). Allerdings kann angenommen werden, dass sich die Effekte der Posttraining-Selbstwirksamkeit in 
wartung bzgl. erwartetem Trainingsnutzen für Arbeitsbewältigung und Karriere metaanalytisch bestätigt (Blume et al., 2010). Zudem wurden auch distalere Faktoren, die Motivation bedingen (wie z. B. Job Involvement bzw. Commitment) als Transferfaktoren identifiziert (Blume et al., 2010).

Da für motivationalen Aspekte unterschiedliche Bezeichnungen verwendet werden (z. B. wird Transferintention häufig von Transfermotivation abgegrenzt jedoch durch ähnliche Items erfasst), werden die Befunde, abhängig von der Operationalisierung nach gängigen Definitionen zusammengefasst. Lernmotivation oder Lernzielorientierung ist dabei definiert als überdauernde Persönlichkeitseigenschaft, Leistungsverhalten auf den Zweck der Kompetenzentwicklung auszurichten und Anforderungen als Lerngelegenheiten zu betrachten (Dweck, 1986). Trainingsmotivation bezieht sich demgegenüber auf Richtung, Intensität und Dauer der Anstrengung sich Fertigkeiten und Wissen im Rahmen eines spezifischen Trainings anzueignen (Noe \& Wilk, 1993). Transfermotivation bestimmt die „Richtung, Intensität und Dauer der Anstrengung, im Training gelernte Fertigkeiten und Wissen im Arbeitsumfeld nutzbar zu machen“ (Kauffeld et al., 2008, S. 53). Selbstwirksamkeit ist definiert als „Überzeugung, dass man generell in der Lage ist, seine Leistung zu ändern, wenn man es will“ (Kauffeld et al., 2008, S. 54). Das dazu verwandte Konstrukt der Leistungserwartung bezieht sich auf die Überzeugung, dass Transferanstrengungen zu besserer Arbeitsleistung führen (Kauffeld et al., 2008). Demgegenüber ist Ergebniserwartung definiert als „Erwartung, dass Änderungen in der Arbeitsleistung zu für den Teilnehmenden erstrebenswerten Ergebnissen führen“ (Kauffeld et al., 2008, S. 54).

Generell wurde aufgezeigt, dass diese zahlreichen motivationalen Konstrukte zwar verschieden sind, sich aber wechselseitig bedingen und hohe Zusammenhänge untereinander aufweisen (Bauer et al., 2016; Gegenfurtner et al., 2016). Metaanalytisch zeigte davon die Transfermotivation den höchsten Zusammenhang mit Transfer, auch wenn zahlreiche Studien die Trainingsmotivation zur Transfervorhersage wie in dem Modell von Colquitt et al. (2000) präferierten. Zudem wurde ein noch höherer Zusammenhang von Transfermotivation mit der unmittelbaren Trainingsreaktion (Zufriedenheits- und Nützlichkeitseinschätzungen) als mit Transfer identifiziert (Bauer et al., 2016). Nützlichkeitseinschätzungen zeigten wiederum einen relativ hohen Zusammenhang mit Transfer, während Zufriedenheitseinschätzungen wenig Vorhersagekraft für Transfer besitzen. Aus den Ergebnissen von Bauer et al. (2016) kann insgesamt abgeleitet werden, dass Trainings, die als gut und nützlich bewertet werden, nicht nur mit Transfermotivation, sondern auch mit Transfer positiv zusammenhängen. Zudem zeigten motivationale

der Transfermotivation widerspiegeln, weshalb solch eine Ausdifferenzierung vermutlich keinen allzu großen Mehrwert bringt. 
Aspekte insbesondere bei interaktionalen Führungskräftetrainings (vs. „Closed-Skills“Trainings) einen hohen Transfereffekt (Blume et al., 2010).

Als weitere Faktoren, die Transfermotivation bedingen, wurde insbesondere die soziale Unterstützung von Organisation, Vorgesetzten und Kollegen metaanalytisch bestätigt (Hughes et al., 2019). Vorgesetztenunterstützung zeigte davon den geringsten Einfluss auf Transfermotivation und wirkte eher über positive Einstellungen zur Trainingsmaßnahme als durch Verhalten (Reinhold et al., 2018).

Unter den veränderbaren Teilnehmendeneigenschaften im Trainings- und Transferprozess kann auch das Kompetenzniveau bezüglich der Trainingsinhalte eingeordnet werden. Obwohl Kompetenzentwicklung bzw. Lernen bereits in den Basismodellen als notwendige Voraussetzung und zentraler Mediator im Transferprozess betrachtet (Baldwin \& Ford, 1988; Kirkpatrick, 1967; Noe \& Schmitt, 1986) und metaanalytisch als entscheidender Transferfaktor auch für Führungskräftetrainings bestätigt wurde (Bauer et al., 2016; Blume et al., 2010; Colquitt et al., 2000), wird diese Variable in prozessorientierten Evaluationsstudien und dessen Metaanalysen relativ selten zur Erklärung von Transfer berücksichtigt.

\section{Neuere Einzelbefunde von Transferdeterminanten}

Aktuelle prozessorientierte Transferstudien analysierten die Zusammenhänge zwischen transferförderlichen Teilnehmendenmerkmalen und gängigen Kriterien für Trainingswirksamkeit - Trainings- (Reaktion und Lernen) sowie Transfererfolg (Transferverhalten und dessen resultierenden Leistungseffekte bzw. Resultate) - um daraus auf den Wirkmechanismus im Transferprozess zu schließen. Eine qualitative Analyse von Lee, Jeon, Kim und Lee (2017) identifizierte durch Interviews mit den 10\% erfolgreichsten Teilnehmenden bzgl. Transfereinschätzung erfolgskritische Teilnehmendeneigenschaften: 1) Trainingsmotivation, 2) persönliche Bedeutungszuordnung zum Trainingsinhalt, 3) Transfermotivation, 4) aktive Trainingsteilnahme, 5) Verwendung von Trainingsunterlagen und 6) Langzeitorientierung bezüglich Trainingswirksamkeit. Eine Übersicht über aktuelle quantitative Befunde zeigt Tabelle 2 ab Seite 53. Dabei kommt der Transfermotivation als zentrale Determinante des Transferprozesses große Beachtung zu. Daher werden nachfolgend erst die Erkenntnisse über Transfermotivation zusammengefasst, bevor die übrigen Befunde zu den vier Ebenen der Trainingswirksamkeit beschrieben werden. 


\section{Transfermotivation ${ }^{16}$}

Der meistbestätigte Zusammenhang prozessorientierter Transferforschung ist die Vorhersagekraft von Transfermotivation nach Trainings für Transfererfolg (z. B. Govaerts, Kyndt \& Dochy, 2018; Lee, Lee, Lee \& Park, 2014; Seiberling \& Kauffeld, 2017; Vignoli \& Depolo, 2019). Dieser besteht auch nach Kontrolle des Einflusses anderer zentraler Transferdeterminanten (Zamani, Ataei \& Bates, 2016). In vielen Studien wurde dabei eine Mediatorrolle der Transfermotivation bei der Transferwirkung weiterer Teilnehmendeneigenschaften (Bhatti, Ali, Isa \& Battour, 2014; Cheng, Sanders \& Hampson, 2015; Iqbal \& Dastgeer, 2017; Vignoli \& Depolo, 2019), der Trainingsgestaltung (Grohmann, Beller \& Kauffeld, 2014; Muduli \& Raval, 2018; Seiberling \& Kauffeld, 2017) oder des Arbeitsumfelds (Chauhan, Ghosh, Rai \& Shukla, 2016; Massenberg \& Kauffeld, 2015; Massenberg, Spurk \& Kauffeld, 2015; Ng, 2015; Ng \& Ahmad, 2018; Schindler \& Burkholder, 2016) bestätigt. Andererseits wurden in Übereinstimmung mit den metaanalytischen Befunden Moderationseffekte aufgezeigt: Transfermotivation mediierte den Zusammenhang zwischen Peer- sowie Vorgesetztenunterstützung und Transfer nur bei Teilnehmenden mit hohem Engagement (Brahma \& Chakraborty, 2019). Der Zusammenhang zwischen Lernmotivation und Transfer war größer, wenn Teilnehmende bessere Nützlichkeits- und Zufriedenheitseinschätzungen abgeben (Kodwani \& Prashar, 2019b). Zudem wurden Zusammenhänge zwischen Transfermotivation und den übrigen drei Ebenen der Trainingswirksamkeit gefunden: Reaktions- (z. B. Seiberling \& Kauffeld, 2017), Lern- (z. B. Iqbal \& Dastgeer, 2017) sowie Resultatebene (Sua Wui, Mohd Haizam Mohd \& Chong Aik, 2018).

Prädiktoren für Transfermotivation wurden sehr umfassend erforscht. Sahoo und Mishra (2019) konnten durch Teilnehmendeneigenschaften, Inhaltsrelevanz und Trainingsbewertung 46\% der Varianz von Transfermotivation aufklären, wobei Inhaltsrelevanz den größten Zusammenhang mit Transfermotivation zeigte. Cheng et al. (2015) erklärten die Rolle der Transfermotivation im Transferprozess mit der Theorie des geplanten Verhaltens nach Ajzen $(1985,1991)$ und zeigten, dass theoriekonform die drei übergeordneten Prädiktoren - Einstellung, wahrgenommene Norm (Transfererwartungen relevanter Interaktionspartner bzw. der Organisation) und wahrgenommene Verhaltenskontrolle bezüglich Transfer - Transfermotivation maßgeblich vorhersagen. Als konkrete Prädiktoren für Transfermotivation wurden darüber hinaus folgende Variablen identifiziert: Arbeitserfahrung und Alter zeigten einen negativen Zusammenhang mit Trans-

\footnotetext{
${ }^{16} \mathrm{Da}$ Transferintention und -volition wie Transfermotivation operationalisiert wird, werden die Be-
} funde dieser Variablen unter „Transfermotivation“ zusammengefasst. 
fermotivation (Brahma \& Chakraborty, 2019). Positive Vorhersagekraft für Transfermotivation zeigten neben ihrem Ausgangswert vor Trainings auch überdauernde Persönlichkeitseigenschaften (Gewissenhaftigkeit, Proaktivität, Extraversion und Verträglichkeit), Commitment bzw. Arbeitsengagement, Lern- und Trainingsmotivation, Selbstbewertung, Selbstwirksamkeit, Selbstregulation bzw. Einsatz von Lernstrategien, Bewusstsein über Entwicklungsbedarf, Vorwissen, Erwartungsklarheit, erwartete Anwendungsmöglichkeit, Leistungs- und Ergebniserwartung, affektive Reaktion (Zufriedenheitseinschätzung des Trainings), transferförderliche Trainingsgestaltung, Kompetenz, Kollegen- sowie Vorgesetztenunterstützung (Alvelos, Ferreira \& Bates, 2015; Arasanmi, Wang \& Singh, 2017; Bhatti et al., 2014; Brahma \& Chakraborty, 2019; Chauhan et al., 2016; Grohmann et al., 2014; Hinrichs, 2014; Iqbal \& Dastgeer, 2017; Y. H. Kim, Ong \& Chung, 2014; Lauzier \& Mercier, 2018; Lee et al., 2014; Lourenco \& Ferreira, 2019; Madagamage, Warnakulasooriya \& Wickramasuriya, 2014; Martins, Zerbini \& Medina, 2019; Massenberg \& Kauffeld, 2015; Massenberg et al., 2017; Massenberg et al., 2015; Ng \& Ahmad, 2018; S. Park, Kang \& Kim, 2018; Roberts, Rogers, Thomas \& Spitzmueller, 2018; Sahoo \& Mishra, 2019; Vignoli \& Depolo, 2019; Wen \& Lin, 2014a, 2014b).

Zudem wurden dahinterliegende Wirkzusammenhänge identifiziert: Lernmotivation mediierte den Einfluss von Proaktivität auf Transfermotivation, wobei mit zunehmender Gewissenhaftigkeit der Zusammenhang zwischen Proaktivität und Lernmotivation geringer wurde (Roberts et al., 2018; Vignoli \& Depolo, 2019). Trainingsmotivation mediierte den Zusammenhang zwischen Lernmotivation sowie Bewusstsein über Entwicklungsbedarf sowie Selbstwirksamkeit und Transfermotivation (S. Park et al., 2018; Wen \& Lin, 2014b). Der Zusammenhang zwischen Selbstwirksamkeit und Transfermotivation zeigte sich nur bei hohen Ausprägungen transformationaler Führung (Vignoli, Mariani, Guglielmi \& Violante, 2018). Soziale Unterstützung moderierte schließlich den Zusammenhang zwischen Trainingsmotivation und Transfermotivation: Je größer die Unterstützung, desto größer der Zusammenhang (Lauzier \& Mercier, 2018).

Zur Arbeitsumgebung wurden insbesondere Kollegen- und Vorgesetztenunterstützung als Prädiktoren von Transfermotivation untersucht und bestätigt (z. B. Brahma \& Chakraborty, 2019; Lee et al., 2014). Während Kollegenunterstützung einen direkten Einfluss auf Transfermotivation zeigte (Massenberg et al., 2017), wurde der Zusammenhang zwischen Vorgesetztenunterstützung und Transfermotivation von Lernmotivation (S. Park et al., 2018) und von ihrem Ausgangswert (Massenberg et al., 2017) mediiert. Ein Befund legt den Schluss nahe, dass Vorgesetztenunterstützung nur bei Teilnehmenden mit geringem Vorwissen einen Einfluss auf Transfermotivation hat (Y. H. Kim et al., 2014). Zudem hat zu viel Unterstützung Transfermotivation gehemmt (Y. Park, Lim \& Chang, 2017). 
Der Zusammenhang zwischen transferförderlicher Trainingsgestaltung und Transfermotivation wurde von sozialer Unterstützung (Alvelos et al., 2015; Chauhan, Ghosh, Rai \& Kapoor, 2017) und Selbstwirksamkeit (Bhatti et al., 2014) mediiert. Der Zusammenhang zwischen Inhaltsrelevanz des Trainings und Transfermotivation wurde dagegen von der affektiven Reaktion auf das Training (Zufriedenheitseinschätzung) mediiert (Bhatti et al., 2014). Schließlich zeigte sich noch ein Moderationseffekt der Motivationsart: Der Zusammenhang zwischen Teilnahmefreiwilligkeit und Transfermotivation bestand nur bei autonomer (intrinsischer) aber nicht bei kontrollierter Transfermotivation (Curado, Henriques \& Ribeiro, 2015).

\section{Reaktion}

Eine Vorhersagekraft der Reaktionsebene (Zufriedenheits- und Nützlichkeitseinschätzungen) zeigte sich für Transfermotivation (z. B. Alvelos et al., 2015; Bhatti et al., 2014; Grohmann et al., 2014; Seiberling \& Kauffeld, 2017) sowie Lernen, Transfer und dessen Resultate (S. Kim, Park, Lavelle, Kim \& Chaudhuri, 2019; Lourenco \& Ferreira, 2019; Martins et al., 2019). Dabei wurde auch eine Mediatorrolle der Reaktion im Zusammenhang zwischen Trainingsmotivation bzw. „off-the-job Klassenzimmersetting“ und Transfererfolg identifiziert (S. Kim et al., 2019; Kodwani \& Prashar, 2019b; Martins et al., 2019). Zudem zeigte sich, wie bereits im Abschnitt zur Transfermotivation (ab Seite 48) erwähnt, auch eine Moderatorrolle von Trainingsreaktion: Der Zusammenhang zwischen Trainingsmotivation und Transfer ist größer, wenn Teilnehmende bessere Nützlichkeitsund Zufriedenheitseinschätzungen abgeben (Kodwani \& Prashar, 2019b). Schließlich konnte der Einsatz von Lernstrategien und Selbstregulation nach dem Training durch Trainingsreaktionen erklärt werden (Martins et al., 2019).

Als Prädiktoren für Trainingsreaktionen wurden neben Trainingsmotivation (S. Kim et al., 2019) auch Kollegen-, Vorgesetzten- und Organisationsunterstützung identifiziert (Alvelos et al., 2015; Martins et al., 2019). Vorgesetztenunterstützung wies insbesondere einen positiven Zusammenhang mit erwarteter Nützlichkeit auf, allerdings hemmte zu viel Unterstützung Effekte der Transfermotivation (Y. Park et al., 2017). Zudem sagte eine transferförderliche und autonomieunterstützende Trainingsgestaltung Nützlichkeitseinschätzungen vorher. Berufserfahrung und wahrgenommene Unterstützung durchs Management zeigten dabei einen moderierenden Effekt: Je mehr Berufserfahrung und Managementunterstützung, desto größer der Zusammenhang zwischen autonomieunterstützender Trainingsgestaltung und Nützlichkeitseinschätzung (Cappetta \& Paolino, 2015). Affektive Reaktion mediierte den Zusammenhang zwischen Inhaltsvalidität des Trainings und Transfermotivation (Bhatti et al., 2014). 


\section{Lernen}

Eine Vorhersagekraft von Lernen wurde nur in wenigen aktuellen Studien untersucht, wobei Effekte auf Transfer identifiziert wurden (Bhatti et al., 2014; Govaerts et al., 2018; Homklin, Takahashi \& Techakanont, 2014; S. Kim et al., 2019; Li \& Wiriyakitjar, 2018; Lourenco \& Ferreira, 2019). Während Transfermotivation diesen Zusammenhang mediierte (Iqbal \& Dastgeer, 2017) wirkte Kollegenunterstützung moderierend darauf ein: Bei hoher Peerunterstützung war die Transferwirkung von Lernen größer (Homklin et al., 2014). Zudem wurde auch eine Mediatorrolle von Lernen im Zusammenhang zwischen Trainingsmotivation, Belohnungserwartung sowie Vorgesetzteninvolvierung und Transfer aufgezeigt (Bhatti et al., 2014; Govaerts et al., 2018). Die Befunde weisen insgesamt darauf hin, dass Kompetenzzuwachs ein notwendiges aber nicht hinreichendes Kriterium der Trainingswirksamkeit darstellt.

Als Prädiktoren für Lernerfolg wurden die Teilnehmendeneigenschaften, Trainingsmotivation (Govaerts et al., 2018; Li \& Wiriyakitjar, 2018) und Trainingsreaktionen (S. Kim et al., 2019) identifiziert. Zudem zeigten Kollegen-, Vorgesetzten- und Organisationsunterstützung positive Zusammenhänge mit Lernen (Homklin et al., 2014; Li \& Wiriyakitjar, 2018). Lernförderliche Vorgesetztenunterstützung konnte durch Transferverantwortung und Involvierung im Trainingsprozess spezifiziert werden (Govaerts et al., 2018). Zudem förderte eine transferförderliche Trainingsgestaltung inklusive Bedarfsanalyse und Evaluation den Lernerfolg (Li \& Wiriyakitjar, 2018).

\section{Transferverhalten}

Eine Vorhersagekraft von Transferverhalten auf Resultate (wie Leistung, oder monetäre Ergebnisgrößen) wurde ebenfalls nur in wenigen aktuellen Studien untersucht, dort aber auch bestätigt (Diamantidis \& Chatzoglou, 2014; Martins et al., 2019; Peters et al., 2014; Shen \& Tang, 2018; Zumrah, 2015).

Forschungsarbeiten zu Prädiktoren von Transfer gibt es dagegen sehr viele. Trainingstransfer ist ein vielschichtiger Prozess, der von vielen Faktoren bestimmt wird, die eher simultan miteinander interagieren als lineare Zusammenhänge abzubilden (Massenberg et al., 2017; Y. Park et al., 2017). Für die meisten Transferprädiktoren wurden daher vorwiegend indirekte Zusammenhänge mit Transfer identifiziert, die in den bisher dargestellten Befunden zusammengefasst sind. Daraus kann abgeleitet werden, dass transferförderliche Teilnehmendenmerkmale, Arbeitsumgebung und Trainingsgestaltung vorwiegend indirekt auf Transfer wirken - insbesondere mediiert durch Lernen und Transfermotivation (z. B. Bhatti et al., 2014; Cheng et al., 2015; Govaerts et al., 2018; Lee et al., 2014; Li \& Wiriyakitjar, 2018). Wobei Kollegenunterstützung beim Zusammenhang 
zwischen Lernen und Transfer eine positive moderierende Wirkung zeigte (Homklin et al., 2014). Darüber hinaus kann theoretisch auch die Notwendigkeit von Anwendungsmöglichkeiten für Transfer als direkte situationale Transferdeterminante abgeleitet werden (siehe Abschnitt 2.5 ab Seite 24). Dieser Zusammenhang wurde auch von aktuellen Studien bestätigt (Muduli \& Raval, 2018; Renta-Davids, Jiménez-González, FandosGarrido \& González-Soto, 2014; Schindler \& Burkholder, 2016; Zamani et al., 2016).

Zudem gibt es noch einzelne Befunde zu Variablen, die noch nicht klar in den Transferprozess eingeordnet werden können, da sie nicht in Zusammenhang mit den zentralen Transferprädiktoren untersucht wurden. Dies gilt beispielsweise für Transferverantwortung (Freitas, Silva \& Santos, 2019), Anwendung von Lernstrategien (Martins et al., 2019), Selbstwert (Sua Wui et al., 2018), Bedürfnis nach kognitiver Beanspruchung (Mensmann \& Frese, 2019), Feedbacksuchverhalten (Diamantidis \& Chatzoglou, 2014; Sparr et al., 2017) oder Arbeitszufriedenheit (Islam \& Ahmed, 2018; Shen \& Tang, 2018; Zumrah \& Boyle, 2015). Allerdings liegt auch hier die Vermutung nahe, dass diese Variablen eher indirekt auf Transfer wirken. Eine vorwiegend direkte Transferwirkung wird daher nur für Transfermotivation, Kompetenzzuwachs und Anwendungsmöglichkeiten angenommen.

\section{Resultate}

Da Resultate (z. B. Arbeitsleistung und Ergebnisgrößen) die letzte Wirksamkeitsebene im Trainings- und Transferprozess darstellen, werden hierfür nur Befunde über Prädiktoren der Transferresultate zusammengefasst. Allerdings gibt es hierzu nur wenige Studien. Neben Transferverhalten (siehe oben), wurden die Teilnehmendeneigenschaften Bewusstsein über Entwicklungsbedarf, Trainingsmotivation, Trainingsreaktionen und Anwendung von Lernstrategien als Prädiktoren identifiziert (Martins et al., 2019; S. Park et al., 2018). Zudem wurden Effekte von Organisations-, Vorgesetzten- und Kollegenunterstützung (Martins et al., 2019; Peters et al., 2014; Shen \& Tang, 2018; Zumrah, 2015), Anwendungsmöglichkeit, Leistungsrückmeldung (Feedback) und Organizationales Commitment (Miiro, Matsiko \& Mazur, 2014; Peters et al., 2014) sowie transferförderlicher Trainingsgestaltung (Diamantidis \& Chatzoglou, 2014) auf Resultate gefunden. Allerdings zeigten mehrere Studien, dass Transfer diese Zusammenhänge mediiert (z. B. Shen \& Tang, 2018; Zumrah, 2015). Darüber hinaus wurde Arbeitszufriedenheit als eigenständiger Prädiktor für Arbeitsleistung identifiziert (Shen \& Tang, 2018). 


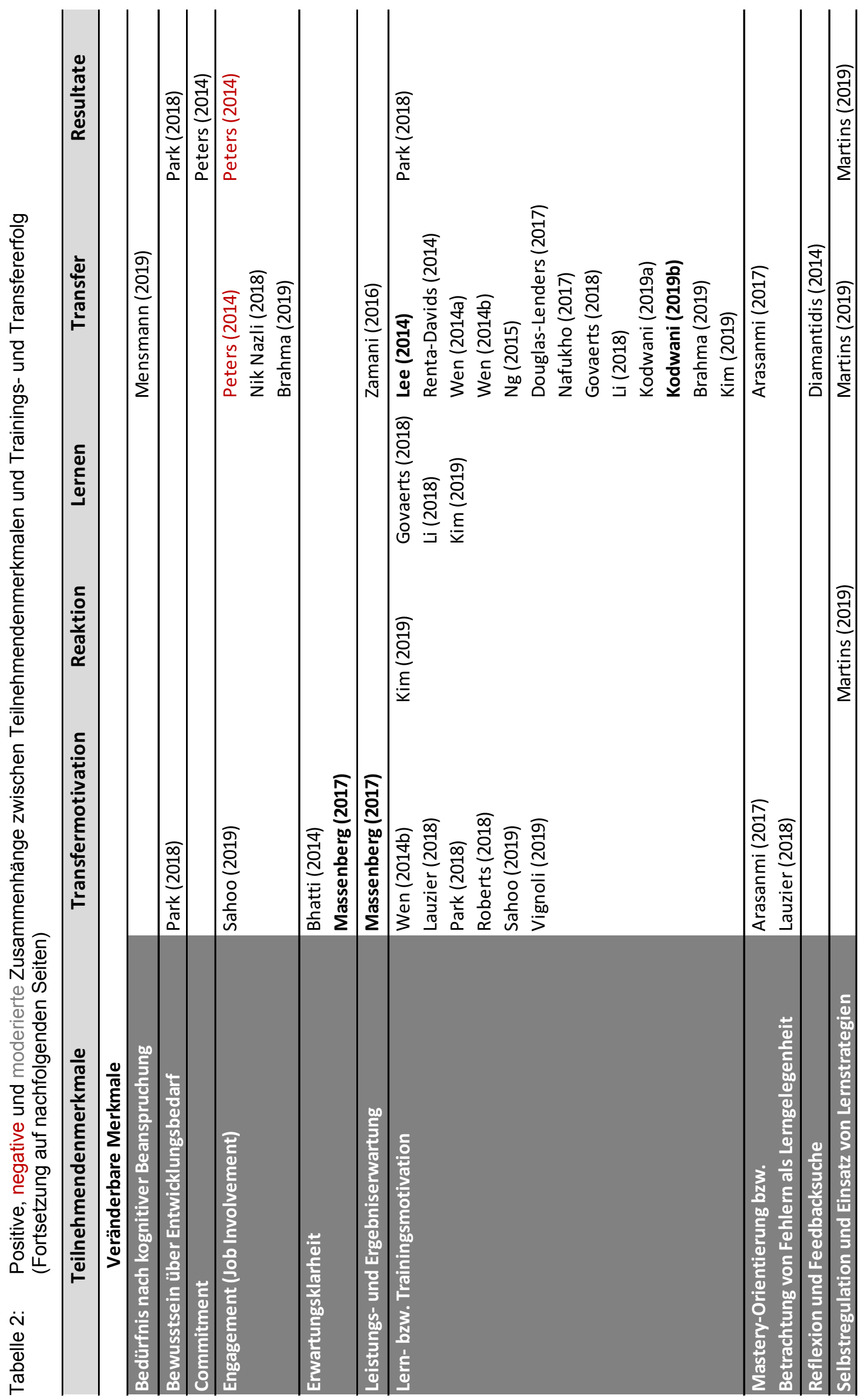




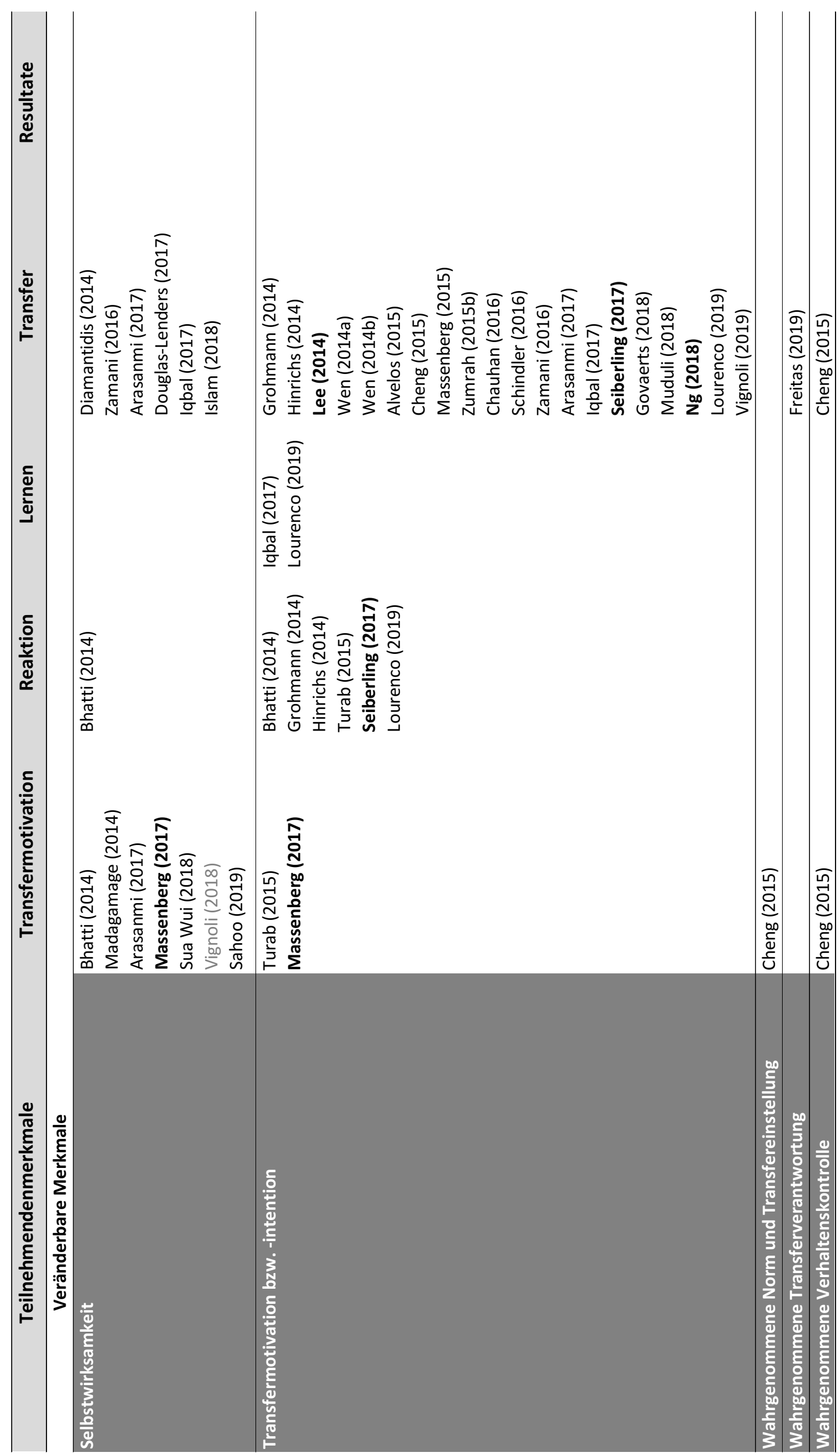




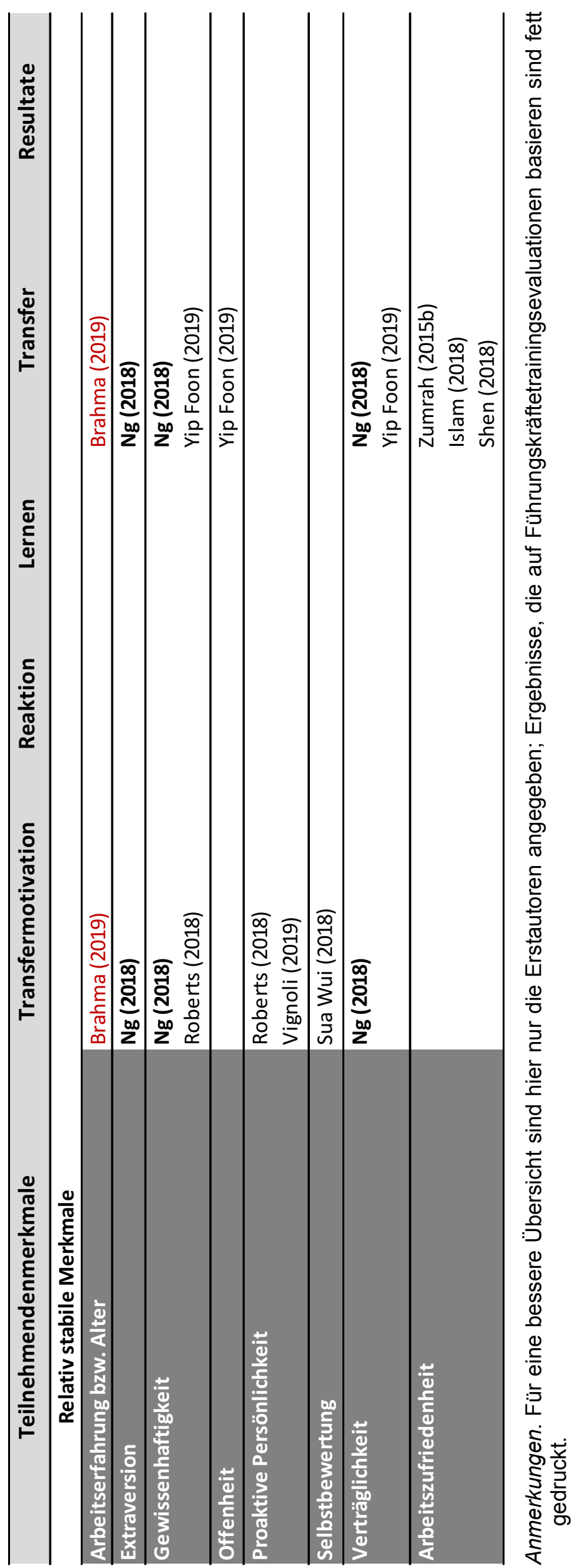




\section{Transferförderliche Arbeitsumgebung}

\section{Metaanalytisch bestätigte Transferdeterminanten}

Die Einteilung der Arbeitsumgebungsfaktoren von Baldwin und Ford (1988) in Unterstützung und Anwendungsmöglichkeit kann auch anhand aktueller Reviews als sinnvoll erachtet werden (Ford et al., 2018). Obwohl gerade die Anwendungsmöglichkeit als entscheidende Voraussetzung für Transfer betrachtet wird, werden in Evaluationsstudien eher Unterstützungsfaktoren untersucht und weiter differenziert. Daher konnte der Einfluss von Anwendungsmöglichkeit trotz seiner Relevanz nicht metaanalytisch überprüft werden (Blume et al., 2010; Hughes et al., 2019). So beziehen sich die metaanalytisch bestätigten Befunde zur Arbeitsumgebung auf Unterstützung durch Transferhinweise und Transferfolgen. Basierend auf dem Basismodell des Transferklimas von Rouiller und Goldstein (1993) sowie Metaanalysen (Blume et al., 2010; Hughes et al., 2019; Reinhold et al., 2018) erscheint die Differenzierung in Organisations-, Vorgesetzten- und Kollegenunterstützung sowie Transferkonsequenzen für diesen Einflussbereich sinnvoll. Alle vier Unterstützungsfaktoren wurden metaanalytisch bestätigt. Bezüglich Transferkonsequenzen wurden spezifisch Feedback und Vorgesetztensanktionen, also die negative Reaktion durch Vorgesetzte auf Trainingstransfer (invers), als relevante Transferdeterminanten identifiziert (Hughes et al., 2019; Reinhold et al., 2018). Von den drei Unterstützungsquellen zeigte Kollegenunterstützung mit $R^{2}=46.8 \%$ die größte Aufklärung der Transfervarianz gefolgt von $\mathrm{R}^{2}=39.5 \%$ durch Vorgesetztenunterstützung und $\mathrm{R}^{2}=$ $14.9 \%$ durch Organisationsunterstützung. Alle drei Unterstützungsquellen besaßen dabei einen inkrementellen Transfereffekt wobei der inkrementelle Effekt von Organisationsunterstützung kontrolliert um Vorgesetzten- und Kollegenunterstützung negativ ausfällt (Hughes et al., 2019). Transfermotivation mediierte die Transferwirkung von Arbeitsumgebungsfaktoren (Ausnahme ist Feedback). Während Kollegenunterstützung den größten Transfereffekt zeigte, beeinflusste Organisationsunterstützung insbesondere die Transfermotivation (Hughes et al., 2019; Reinhold et al., 2018). Transfereffekte sozialer Unterstützung resultierten insbesondere bei Trainings zur Vermittlung von „Open-Skills“ (Blume et al., 2010) und bei typischem (vs. maximalem) Transfer (Huang et al., 2015). 


\section{Neuere Einzelbefunde von Transferdeterminanten}

Wie metaanalytisch aufgezeigt, ist die Arbeitsumgebung wichtig für das „Wollen“ und die „situative Ermöglichung“ von Trainingstransfer. Aktuelle prozessorientierte Transferstudien untersuchten welche konkreten Faktoren eine transferförderliche Arbeitsumgebung ausmachen. Die qualitative Analyse von Lee et al. (2017) identifizierte neben Teilnehmenden- und Trainingseigenschaften drei erfolgskritische Eigenschaften der Arbeitsumgebung: 1) Anwendungsmöglichkeit, 2) Lernkultur und 3) veränderungsorientierte Arbeit. Eine Übersicht über aktuelle quantitative Befunde zu Transferfaktoren der Arbeitsumgebung zeigt Tabelle 3 ab Seite 63. Anhand der metaanalytisch abgebildeten Einteilung in Anwendungsmöglichkeit, Transferunterstützung von Organisation, Vorgesetzten und Kollegen sowie Transferkonsequenzen werden auch die Einzelbefunde nachfolgend zusammengefasst.

\section{Transfermöglichkeit}

Im LTSI werden zwei Aspekte der unmittelbaren Transfermöglichkeit unterschieden: Transferkapazität, definiert als „Ausmaß, in dem der Trainingsteilnehmende zeitliche und Belastungskapazitäten zur Verfügung hat, um neu Gelerntes anzuwenden" (Kauffeld et al., 2008, S. 53) und Möglichkeit der Anwendung, definiert als „Grad, inwieweit Materialien, Werkzeuge, Budgets, etc. bereitstehen, um das Gelernte anwenden zu können“ (Kauffeld et al., 2008, S. 53). Daraus zusammengefasst definieren wir im Kontext der vorliegenden Arbeit „Transfermöglichkeit“ als zeitliche, inhaltliche und materielle Voraussetzungen, um trainierte Kompetenzen anwenden zu können.

Die Vorhersagekraft von Transfermöglichkeit in diesem Sinne wurde für Transferverhalten (Muduli \& Raval, 2018; Renta-Davids et al., 2014; Schindler \& Burkholder, 2016; Zamani et al., 2016) und -resultate (Miiro et al., 2014) auch über den Effekt anderer Transferdeterminanten des LTSI hinaus bestätigt. Zudem zeigte erwartete Transfermöglichkeit vor dem Training eine Vorhersagekraft für Transfermotivation (Massenberg et al., 2017). Für Transfermöglichkeit wurde auch ein inkrementeller Transfereffekt über den Einfluss anderer Transferdeterminanten hinaus nachgewiesen (Miiro et al., 2014; Nafukho, Alfred, Chakraborty, Johnson \& Cherrstrom, 2017; Renta-Davids et al., 2014; Zamani et al., 2016). Im Gegensatz zu Transferunterstützung und -konsequenzen wurde die Transferwirkung von Anwendungsmöglichkeit nicht durch Transfermotivation mediiert (Schindler \& Burkholder, 2016). Schließlich zeigte Yip Foon (2019), dass Anwendungsmöglichkeit als Moderator im Zusammenhang zwischen transferförderlichen Persönlichkeitseigenschaften (Gewissenhaftigkeit, Offenheit und Verträglichkeit) und Trans- 
fer im Sinne der Trait-Activation-Theorie (Tett \& Burnett, 2003) wirkt: Bei geringer Anwendungsmöglichkeit zeigten transferförderliche Persönlichkeitseigenschaften eine größere Transferwirkung.

Welche Prädiktoren auf Anwendungsmöglichkeit wirken wurde bisher kaum untersucht. Studien zeigten aber, dass eine transferförderliche Trainingsgestaltung mit Anwendungsmöglichkeit positiv zusammenhängen (Massenberg et al., 2017; Muduli \& Raval, 2018; Nafukho et al., 2017; Renta-Davids et al., 2014). Allerdings konnte aufgrund der Studiendesigns nicht geklärt werden, ob Trainings eher transferförderlich wahrgenommen werden, wenn mehr Anwendungsmöglichkeiten existieren oder ob mehr Anwendungsmöglichkeiten in Folge eines transferförderlichen Trainings von den Teilnehmenden wahrgenommen werden. Dennoch schlossen Muduli und Raval (2018) aus ihren Daten, dass die Transferwirkung von Anwendungsmöglichkeit durch transferförderliche Trainingsgestaltung mediiert wird. Darüber hinaus wird in der Transferforschung der Einfluss von Organisations-, Vorgesetzten- und Kollegenunterstützung sowie die durch Transferkonsequenzen geförderte Transferverantwortung der Teilnehmenden als Prädiktoren für wahrgenommene bzw. von Trainingsteilnehmenden geschaffene Anwendungsmöglichkeit theoretisch hergeleitet (z. B. Grossman \& Burke-Smalley, 2018). Es kann angenommen werden, dass wahrgenommene Transferverantwortung der Trainingsteilnehmende anhaltende Transfermotivation und Fokussierung auf Transmöglichkeiten fördert und dass daraus intensivere Bemühungen resultieren Anwendungsmöglichkeiten zu finden und zu nutzen (Cheng et al., 2015; Grossman \& Burke-Smalley, 2018). Umfassendere Untersuchungen dazu stehen allerdings noch aus.

\section{Transferunterstützung}

Für aussagekräftige Handlungsimplikationen ist es wichtig die Transferunterstützung der Arbeitsumgebung zu spezifizieren und insbesondere Organisations-, Vorgesetzten- und Kollegenunterstützung zu differenzieren (Hughes et al., 2019). Im LTSI werden Vorgesetzten- und Kollegenunterstützung definiert als „Ausmaß, in dem [Vorgesetzte bzw.] gleichgestellte Kollegen das Lernen „on-the-job“ unterstützen und verstärken“ (Kauffeld et al., 2008, S. 53). Organisationsunterstützung durch das Management (z. B. durch Implementierung genereller Personalentwicklungsrichtlinien zur Sensibilisierung für Transferrelevanz und -verantwortung) sind im LTSI nicht enthalten. Eine Definition für Organisationsunterstützung kann aber daraus abgeleitet werden als Ausmaß, in dem die Unternehmensführung das Lernen „on-the-job“ und dadurch Transfer im Arbeitsalltag unterstützt und verstärkt (z. B. durch Richtlinien, Vorbildwirkung oder Steuerungssysteme). Zudem gibt es neben diesen Verhaltens- bzw. aktionsorientierten Unterstützungsformen 
noch die generelle Offenheit für Änderungen in der Arbeitsgruppe. Diese eher einstellungsorientierte Unterstützungsvariable wird im LTSI definiert als „Ausmaß, in dem vorherrschende Normen in der Gruppe die Anwendung von Fertigkeiten und Wissen ermutigen“ (Kauffeld et al., 2008, S. 54). Allerdings haben nur wenige Studien diese Variable mit erfasst, wobei keine oder sogar negative Transfereffekte identifiziert wurden (Govaerts et al., 2018; Miiro et al., 2014; Peters et al., 2014; Zamani et al., 2016). Daher ist die Bedeutung der Offenheit der Arbeitsgruppe im Transferprozess noch weitestgehend unklar. Manche Studien betrachten einstellungs- und aktionsorientierte Vorgesetzten- und Kollegenunterstützung auch in einer zusammengefassten Variablen, dem „Transferklima“ oder der sozialen Unterstützung durch die Arbeitsgruppe. Während Wen und Lin (2014a) nur Zusammenhänge des Transferklimas mit Trainings- und Transfermotivation finden konnten, wiesen andere Studien auch den von Blume et al. (2010) metaanalytisch bestätigten Transfereffekt des Transferklimas nach (z. B. Kodwani \& Prashar, 2019a; Vignoli \& Depolo, 2019). Insgesamt ist aber auch diese Variable nicht klar erforscht, sehr uneinheitlich operationalisiert und für konkrete Handlungsimplikationen ist die fehlende Spezifizierung der organisationalen Akteure suboptimal.

Dagegen ist die Vorgesetztenunterstützung eine sehr umfassend analysierte und spezifizierte Transferunterstützungsvariable. Eine qualitative Studie von Govaerts, Kyndt, Vreye und Dochy (2017) spezifizierte transferförderliche Vorgesetztenunterstützung durch 83 Unterstützungsansätze, die in die 24 übergeordneten Unterstützungskategorien nach Govaerts und Dochy (2014) eingeordnet werden. Diese starke Ausdifferenzierung resultiert allerdings in einer Komplexität, die weder für die Praxis noch für die Forschung handhabbar ist. Auch werden davon nur wenige Ansätze (Trainingsauswahl, Trainingsteilnahme sowie Lern- und Transfer-Feedbackgespräche) in der Praxis häufig eingesetzt. Auch Govaerts et al. (2018) haben nur neun Unterstützungsansätze für ihre qualitativen Analysen herangezogen. Davon zeigte nur der Ansatz „Vorgesetzteninvolvierung und -verantwortungsbewusstsein" eine Transferwirkung (mediiert durch Kompetenzzuwachs). Als spezifische Vorgesetztenunterstützungsformen wurden zudem Trainingszuweisung und Austausch zum Training von Ng (2015) als transferförderlich bestätigt. Zudem wurde ein positiver Zusammenhang transformationaler Führung mit Transfermotivation nachgewiesen (Vignoli et al., 2018). Schindler und Burkholder (2016) zeigten, dass die Vorgesetztenunterstützung in Form von Coaching, Aufgaben- und Umsetzungsunterstützung auch durch Mentoren ersetzt werden kann. Da es vermutlich eine unendliche Fortführung möglicher Unterstützungsformen durch Vorgesetzte (oder andere höhergestellte Bezugspersonen) gibt, könnte eine Kategorisierung nach Zeitpunkt (vor, während oder nach Trainings) der Unterstützung im Trainings- und Transferprozess bzw. Förderziel (z. B. Trainings-, Transfermotivation, Lern- oder Transferverhalten) oder 
eine Differenzierung in transferförderliche Einstellung und Verhalten (siehe auch Hinrichs, 2016) praktikabler sein.

Einzelne aktuelle Befunde bestätigten die metaanalytisch überprüfte Vorhersagekraft von Organisationsunterstützung für die Anwendung von Lernstrategien nach dem Training (Lourenco \& Ferreira, 2019; Martins et al., 2019) sowie für Transfer(motivation) (z. B. Islam \& Ahmed, 2018; Ng \& Ahmad, 2018). Diese gilt auch im Vergleich mit den anderen Unterstützungsebenen als sehr relevant (Martins et al., 2019). Allerdings zeigten Lee et al. (2014), dass der Transfereffekt der Organisationsunterstützung durch Transfermotivation mediiert wird und nur bei „Low Performern“ (Teilnehmende, die in Bezug auf den Trainingsinhalt vor dem Training auf einem geringen Leistungsniveau sind) auftritt. Zudem konnte Organisationsunterstützung in Form einer unternehmensübergreifenden und gelebten Lernkultur ${ }^{17}$, nicht nur Transfer, sondern bereits Transfermotivation, Trainingsreaktion und Lernerfolg vorhersagen (Li \& Wiriyakitjar, 2018; Lourenco \& Ferreira, 2019). Stärker erforscht ist dagegen die Vorhersagekraft von Vorgesetzten- und Kollegenunterstützung für die Anwendung von Lernstrategien nach dem Training (Martins et al., 2019) sowie für Trainings- (z. B. Alvelos et al., 2015; Ng, 2015; Y. Park et al., 2017; Wen \& Lin, 2014a) und Transfermotivation (z. B. Bhatti et al., 2014; Massenberg et al., 2017; Seiberling \& Kauffeld, 2017) und dadurch mediiert auch für Transfer (z. B. Chauhan et al., 2016; Massenberg et al., 2015; Schindler \& Burkholder, 2016). Nach Brahma und Chakraborty (2019) zeigte sich die Transferwirkung aber nur bei Teilnehmenden mit hohem Arbeitsengagement. In einer aktuellen Studie wurde auch die Vorhersagekraft auf Lernen bestätigt (Li \& Wiriyakitjar, 2018). Die Vorgesetztenunterstützung in Form von Motivationsförderung durch Steigerung des erwarteten und wahrgenommenen Trainingsnutzens vor Trainings wurde als zentraler Wirkmechanismus für Transfer identifiziert (Cappetta \& Paolino, 2015; Freitas et al., 2019; Massenberg et al., 2017; Massenberg et al., 2015; Y. Park et al., 2017) - insbesondere bei Softskill-Trainings (Massenberg \& Kauffeld, 2015). Zu viel Vorgesetztenunterstützung wirkte sich jedoch negativ auf Motivation aus (Y. Park et al., 2017) und nur bei Teilnehmenden mit geringem Vorwissen zeigte Vorgesetztenunterstützung einen positiven Einfluss auf Transfermotivation (Y. H. Kim et al., 2014).

Dagegen wirkte Kollegenunterstützung insbesondere nach Trainings transferförderlich und zeigte in dieser Prozessphase eine größere Transferwirkung als Vorgesetztenunterstützung (Chauhan et al., 2016; Massenberg et al., 2017; Massenberg et al., 2015;

\footnotetext{
17 Lernkultur ist dabei definiert als Teilkonstrukt der generellen Organisationskultur bezüglich gelebter Leitlinien und Normen zur Förderung kontinuierlichen Lernens (Sonntag, Schaper \& Friebe, 2005).
} 
Peters et al., 2014). Kollegenunterstützung moderierte dabei den Zusammenhang zwischen Kompetenzzuwachs und Transfer: Bei hoher Peerunterstützung war der Zusammenhang höher (Homklin et al., 2014). Zudem nahm Lauzier und Mercier (2018) eine Moderatorrolle von Transferunterstützung auf allen drei Ebenen im Zusammenhang zwischen Trainings- und Transfermotivation an: Je größer die soziale Unterstützung, desto größer der Zusammenhang. Bei Trainings zur Förderung der Zusammenarbeit bzw. des Klimas in der Arbeitsgruppe, konnte ein schlechtes Klima vor dem Training jedoch auch transferförderlich wirken, indem es die persönliche Relevanz und damit motivationale Aspekte steigerte (Hammer, Truxillo, Bodner, Pytlovany \& Richman, 2019).

Die Identifikation von Prädiktoren von Transferunterstützung ist nicht im Fokus der Transferforschung. Dennoch untersuchte eine Studie von Alvelos et al. (2015) Transferdeterminanten als Prädiktoren von Kollegen- und Vorgesetztenunterstützung (allerdings ohne Differenzierung dieser Unterstützungsebenen). Dabei konnte die Trainingsreaktion bezüglich transferförderlicher Trainingsgestaltung einen substanziellen Teil der Unterstützung nach Trainings vorhersagen. Allerdings basierten die Analysen auf einer Querschnittsuntersuchung, weshalb die Wirkrichtung mit Vorsicht zu interpretieren ist.

\section{Transferkonsequenzen}

Während sich Transferunterstützung auf einstellungs- und verhaltensorientierte Begünstigung bzw. Hinweise verschiedener organisationaler Akteure vor dem Transfer bezieht, beziehen sich Transferkonsequenzen auf die Folgen des Transferverhaltens. Im LTSI werden vier Konsequenzformen unterschieden (Kauffeld et al., 2008, S. 53 f.): 1) Positive Folgen bei Anwendung („Grad, in dem die Anwendung des Trainings in der Arbeit zu positiven Auswirkungen führt“), 2) negative Folgen bei Nicht-Anwendung („Grad, in dem die Nicht-Anwendung der Trainingsinhalte in der Arbeit zu negativen Auswirkungen führt“), 3) Vorgesetztensanktionen („Ausmaß, in dem Teilnehmende negative Reaktionen von ihrem Vorgesetzten wahrnehmen, wenn sie Gelerntes anwenden“) und 4) Feedback („formelle und informelle Rückmeldung über eine individuelle Arbeitsleistung“). Wobei nur Vorgesetztensanktionen bezüglich organisationaler Akteure spezifiziert ist. Wie bereits Metaanalysen betätigten, zeigten auch neuere Studien nur eine Vorhersagekraft von Feedback und Vorgesetztensanktionen für Transfer bzw. Transfermotivation (Miiro et al., 2014; Schindler \& Burkholder, 2016; Zamani et al., 2016). Bhatti et al. (2014) zeigten zudem, dass Annahmen intrinsischer Belohnung als Trainingsfolgen (z. B., dass durch das Training eine Steigerung von Kompetenz oder Selbstbestimmung resultiert) nicht nur Transfermotivation, sondern auch Lernerfolg vorhersagen können. Studien, die auch die anderen Konsequenzformen erfasst haben, konnten deren Transferwirkung nicht bestätigen (z. B. Massenberg et al., 2017; Peters et al., 2014; Zamani 
et al., 2016). Jedoch wurde das bisher eher theoretisch hergeleitete und erst anfänglich quantitativ erforschte Konstrukt „Transferverantwortung" von Teilnehmenden mit den Transferkonsequenzen in Zusammenhang gebracht. Es kann angenommen werden, dass durch positive Transferkonsequenzen den Teilnehmenden auch Transfererwartung bzw. -verantwortung verdeutlicht wird. Wenn Trainingsteilnehmende wissen, dass sie für die Anwendung trainierter Kompetenzen verantwortlich gemacht werden, steigt auch ihre Transfermotivation und Fokussierung auf Transferverhalten beispielsweise durch den Einsatz von Selbstregulationsstrategien und intensivere Bemühungen Anwendungsmöglichkeiten zu finden und zu nutzen (Cheng et al., 2015; Grossman \& BurkeSmalley, 2018). Auch wenn Madagamage et al. (2014) den Zusammenhang zwischen Transferverantwortung und -motivation nicht bestätigen konnten, zeigten Freitas et al. (2019), dass Transferverantwortung von transferförderlichen Reaktionen der Vorgesetzten und Kollegen begünstigt wird und Transfererfolg vorhersagen kann. Zudem weisen die Ergebnisse überraschenderweise darauf hin, dass negative Einstellungen und Verhaltensweisen bezüglich Trainingstransfer von Vorgesetzten (Sanktionen) die persönliche Transferverantwortung der Teilnehmenden verstärkt. Da Prädiktoren von Transferkonsequenzen ebenfalls nicht im Fokus der Transferforschung stehen, gibt es dazu keine weiteren belastbaren Befunde. 


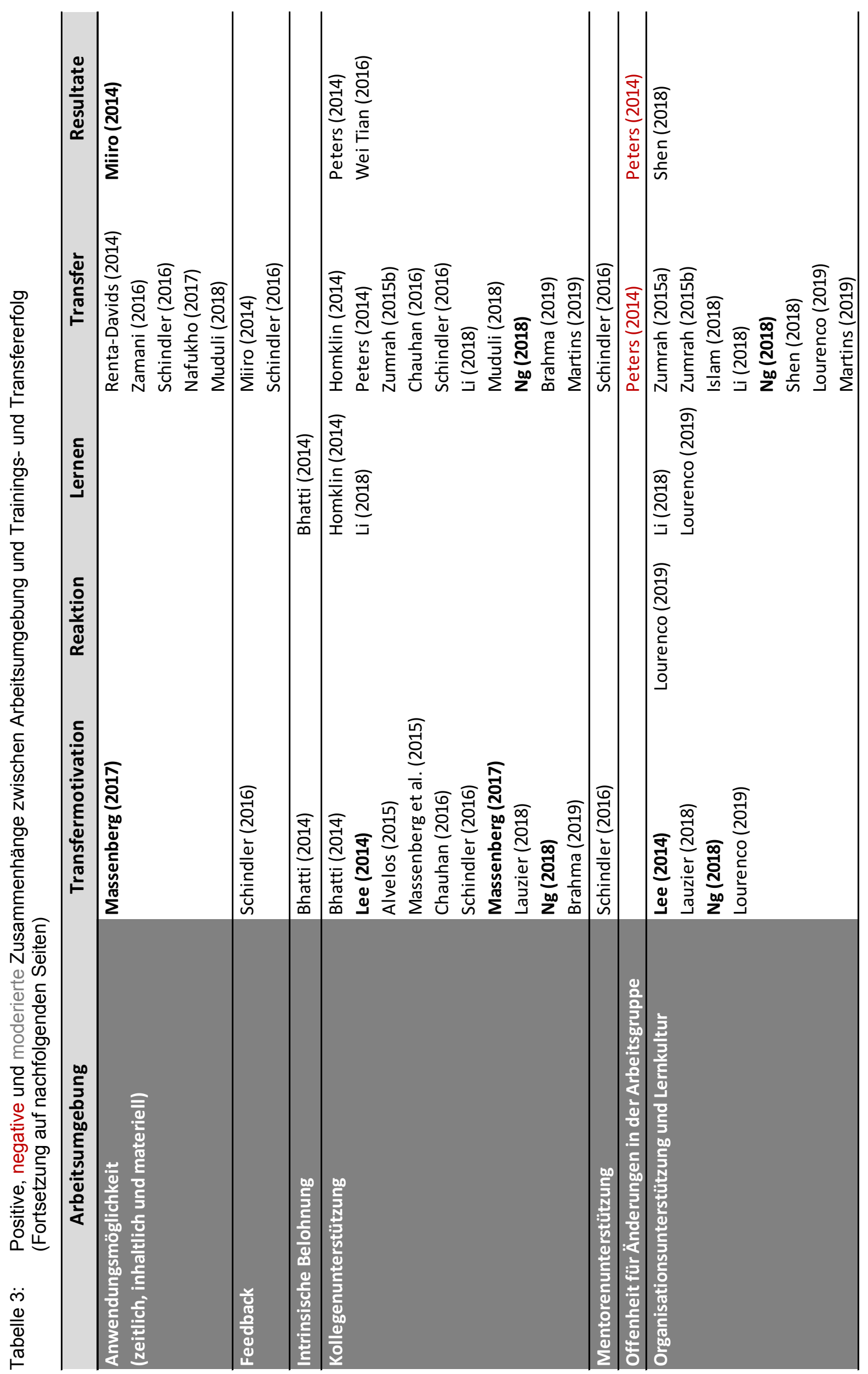




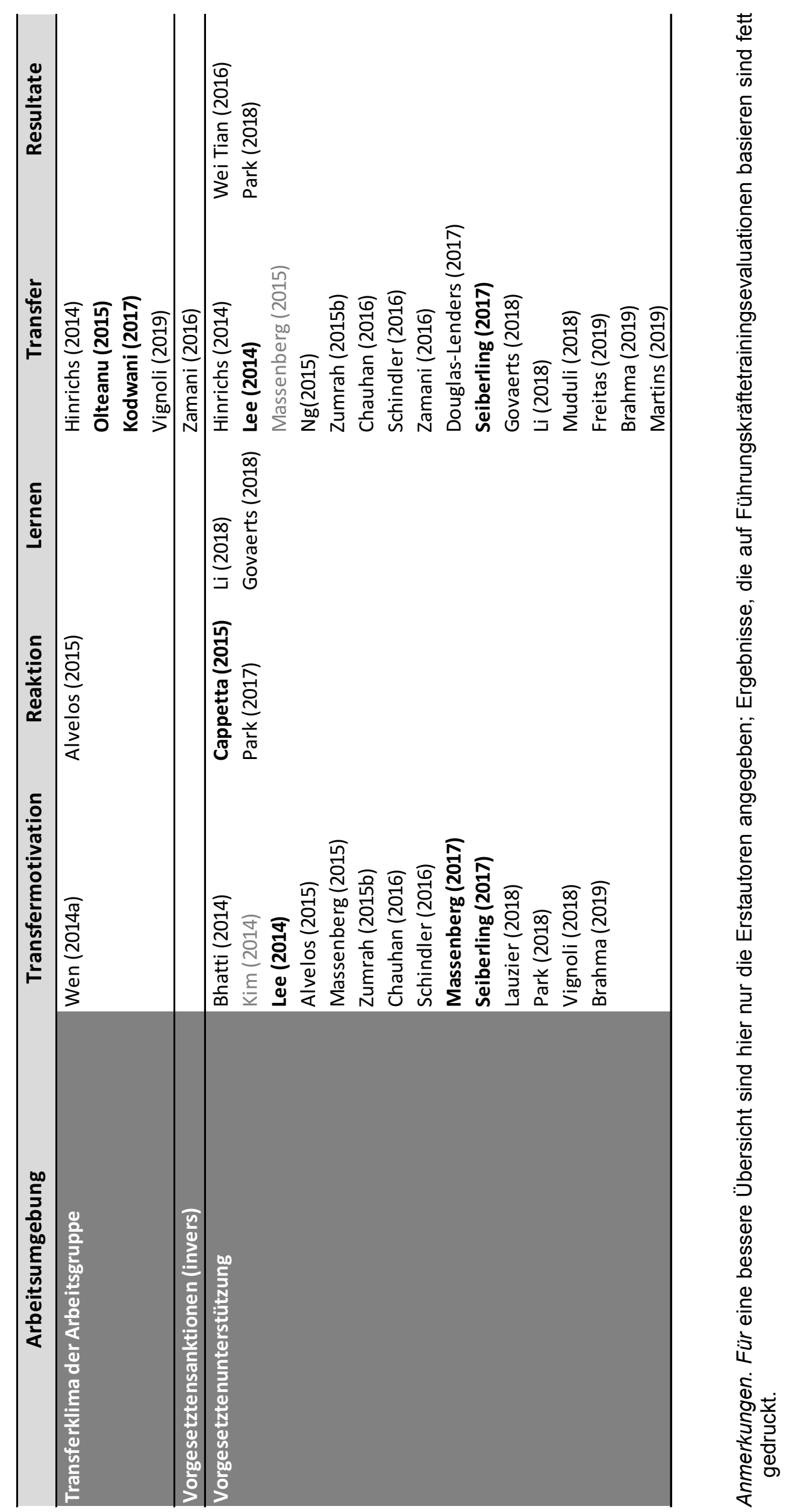




\section{Transferförderliche Trainingsgestaltung}

\section{Metaanalytisch bestätigte Transferdeterminanten}

Welche Faktoren der Trainingsgestaltung transferförderlich wirken wurde über Jahrzehnte hinweg nur bedingt erforscht. Grund hierfür ist, dass Trainingsevaluationen häufig die Transferwirkung unterschiedlicher Trainings zusammengefasst betrachten und Studien nur wenig Informationen zur Trainingsgestaltung geben. So konnten Blume et al. (2010) in ihrer Metaanalyse nur wenige Studien identifizieren, die belastbare Erkenntnisse zu transferförderlicher Trainingsgestaltung machen. Verfügbare Ergebnisse deuteten jedoch darauf hin, dass als nützlich bewertete Trainings von allen betrachteten Transferdeterminanten die größte Vorhersagekraft für Transfer besitzen. Zudem wirkte eine optimistische Vorinformation bezüglich Trainingsrelevanz, -ziel, -inhalt und -methoden durch sogenannte Pre-Traininginterventionen transferförderlich. Es wurde jedoch kein belastbarer Transferzusammenhang von Post-Traininginterventionen zur Förderung von Selbstregulationskompetenzen für Transfer (Rückfallprävention oder Zielsetzung) festgestellt. Darüber hinaus zeigte Gegenfurtner (2011) in seiner Metaanalyse über den Zusammenhang zwischen Transfermotivation und Transfer, dass dieser durch Fokussierung auf Vermittlung deklarativen Wissens und Selbstregulationskompetenzen (vs. Vermittlung prozeduralen Wissens) sowie durch Teilnehmendenzentrierung (vs. Wissenszentrierung) verstärkt werden kann. Gegenfurtner et al. (2016) zeigten zudem, dass bei lernzielorientierten Teilnehmenden eine freiwillige Teilnahmepolitik transferförderlich ist, während bei leistungszielorientierten Teilnehmenden eine Teilnahmeverpflichtung größere Transfereffekte hervorbringt. Weitere Metaanalysen bestätigten die Wirksamkeit von zwei spezifischen Trainingsmethoden: Behavior Modeling Training (siehe auch Abschnitt 2.1 auf Seite 11) wirkte insbesondere bei Vermittlung interaktionaler Kompetenzen (Taylor et al., 2005) und Error Management Training war insbesondere bezüglich vertikalem Transfer Trainingsmethoden überlegen, die keine Explorationsmöglichkeiten und Ermutigung Fehler zu machen und daraus zu lernen bieten (Keith \& Frese, 2008).

Die bereits im Rahmen ergebnisorientierter Trainingsforschung vorgestellte Metaanalyse von Lacerenza et al. (2017) lieferte insbesondere für den Fokus der vorliegenden Arbeit die belastbarste Aussagekraft bezüglich transferförderlicher Gestaltungsaspekte, da sie sich explizit auf Führungskräftetrainings bezog und im Vergleich zu den übrigen Metaanalysen mehr Variablen bezüglich Trainingsgestaltung analysierte. Dabei wurde die Wirksamkeit von evidenzbasierten und praktisch relevanten Trainingskonzep- 
ten mit einer konsequenten Fokussierung auf spezifischen Trainingsbedarf bestätigt. Darauf aufbauend wurden konkrete Gestaltungsaspekte als „Best Practices“ für Transferförderung identifiziert: 1) Durchführung von Bedarfsanalysen zur Identifikation des kontext- und teilnehmendenspezifischen Entwicklungsbedarfs, 2) Einsatz eines passenden Methodenmixes aus Information, Demonstration und Übungsgelegenheiten, 3) Geben von individuellem Feedback, 4) Nutzung von Präsenztrainingformaten, 5) Gewährung einer freiwillige Teilnahmepolitik und 6) zeitlich verteiltes Training über mehrere Sitzungen sowie 7) kombinierte Vermittlung von Fachkompetenzen und Softskills.

\section{Neuere Einzelbefunde von Transferdeterminanten}

In ihrer qualitativen Befragung von Teilnehmenden mit dem größten Transfererfolg identifizierten Lee et al. (2017) ebenfalls kritische Erfolgsfaktoren für die Trainingsgestaltung: 1) Hohe praktische Relevanz der Trainingsinhalte, 2) Verwendung praxisnaher Beispiele, 3) Vermittlung durch Übungsgelegenheiten, 3) Freiwilligkeit bezüglich Aufgabenwahl und -durchführung, 4) ganzheitliche Erfahrungen eingebettet in den Gesamtprozess des Trainingsgegenstandes sowie 5) Austausch und Networking zwischen Teilnehmenden. Aktuelle quantitative Befunde zur Wirksamkeitsüberprüfung transferförderlicher Trainingsgestaltung basieren vorwiegend auf den LTSI-Variablen Transferdesign, definiert als „Ausmaß, in dem das Trainingsdesign Möglichkeiten zum Transfer bietet und in dem die Übungen des Trainings auf die tatsächlichen Arbeitsanforderungen vorbereiten“ und Training-Arbeits-Übereinstimmung, definiert als „Ausmaß, in dem die Trainingsinhalte mit den Anforderungen im Job übereinstimmen“ (Kauffeld et al., 2008, S. 53).

Aktuelle Befunde bestätigten die metaanalytisch identifizierte Transferrelevanz von PreTrainingintervention (Kodwani, 2017; Kodwani \& Prashar, 2019b) Bedarfsanalysen mediiert durch Lernerfolg (Li \& Wiriyakitjar, 2018) und. Darüber hinaus wurden weitere Trainingsmerkmale als transferrelevant identifiziert: Leistung des Trainers (Seiberling \& Kauffeld, 2017), geringe Diversität in der Trainingsgruppe (Creon \& Schermuly, 2019), Freiwilligkeit als Moderator im Zusammenhang zwischen Trainingsmotivation und Transfer (Kodwani \& Prashar, 2019a) bzw. als Prädiktor für intrinsische Transfermotivation (Curado et al., 2015), Förderung selbstgesteuerten Lernens als Prädiktor für Transfermotivation, Trainingsreaktion, Lernen und dadurch auch für Transfer (Cappetta \& Paolino, 2015; Lourenco \& Ferreira, 2019; Sua Wui et al., 2018) als auch die angemessene Trainingsevaluation zur Steigerung von Lernen und Transfer (Kodwani \& Prashar, 2019a; Li \& Wiriyakitjar, 2018). Zudem wurden drei Trainingsmethoden als lern- und transferförderlich zumindest für Softwaretrainings bestätigt: Error 
Management, Behavior Modeling und Teambased Training, wobei Teambased Training den größten Lernerfolg bezüglich prozeduralem Wissen und Transfererfolg zeigte, während Error Management Training den größten Lernerfolg bezüglich deklarativem Wissen bewirkte (Hart, Steinheider \& Hoffmeister, 2019).

Obwohl es nach wie vor kaum Evaluationen von Post-Traininginterventionen gibt, ist anzunehmen, dass auch diese durch Förderung von Selbstmanagementkompetenzen einen transferförderlichen Gestaltungsaspekt darstellen. Setzt man sie ein, um nach der Kompetenzentwicklung deren Anwendungsmöglichkeiten und -barrieren explizit zu reflektieren und Verhaltensabsichten zu formulieren, sollte es sowohl die Transfermotivation als auch das Finden von Anwendungsmöglichkeiten fördern. Zumindest wird diese Annahme auch von aktuellen Reviews gestützt (Rahyuda, Soltani \& Syed, 2018).

\section{Inhaltsrelevanz}

Die Vorhersagekraft von Training-Arbeits-Übereinstimmung bzw. Inhaltsrelevanz wurde nicht nur für Transfermotivation (z. B. Alvelos et al., 2015; Sahoo \& Mishra, 2019) und Trainingsreaktion (Bhatti et al., 2014), sondern auch für Transfer (Y. Park et al., 2017; Renta-Davids et al., 2014) bestätigt. Zudem zeigten Li und Wiriyakitjar (2018), dass eine Bedarfsanalysenbasierung von Trainings, was als Voraussetzung für Inhaltsrelevanz gilt, auch Lernerfolg vorhersagt.

Als Prädiktoren von Inhaltsrelevanz wurden Selbstwirksamkeit, erwartete Trainingsnützlichkeit und Trainingsmotivation identifiziert (Y. Park et al., 2017). Zudem zeigte Inhaltsrelevanz einen Suppressoreffekt auf Aufgabenvariabilität und mediierte den Zusammenhang zwischen lernorientierter Trainingsmotivation sowie Aufgabenkomplexität und Transfer (Renta-Davids et al., 2014).

\section{Transferförderliche Trainingsmethoden}

Zudem wurde die Vorhersagekraft von transferförderlichen Trainingsdesigns, was auch in einigen Studien Inhaltsrelevanz einschließt, für Transfermotivation und alle vier Ebenen der Trainingswirksamkeit nach Kirkpatrick bestätigt (z. B. Martins et al., 2019; Nafukho et al., 2017; Shen \& Tang, 2018). Selbstwirksamkeit mediierte dabei den Zusammenhang zwischen transferförderlicher Trainingsgestaltung und Transfermotivation (Bhatti et al., 2014). Insgesamt weisen die Befunde dabei auf eine Mediatorrolle von Transfermotivation (z. B. Alvelos et al., 2015; Massenberg et al., 2017; Muduli \& Raval, 2018; Seiberling \& Kauffeld, 2017) und Lernen (Li \& Wiriyakitjar, 2018) im Zusammenhang zwischen Trainingsgestaltung und Transfer hin. Zudem wurden Moderationseffekte 
bei den Zusammenhängen transferförderlicher Trainingsgestaltung mit Trainingsreaktionen sowie Transfer identifiziert: Nur bei hoch ausgeprägter Transferunterstützung der Arbeitsumgebung zeigte Trainingsgestaltung einen Zusammenhang mit Transfer (Shen \& Tang, 2018). Der Zusammenhang war umso größer je mehr Vorgesetztenunterstützung wahrgenommen wurde. Somit konnte Vorgesetztenunterstützung suboptimale Trainingsgestaltung teilweise kompensieren (Chauhan et al., 2017). Berufserfahrung und wahrgenommene Unterstützung durchs Management zeigten einen positiven moderierenden Effekt beim Zusammenhang zwischen Trainingsgestaltung und Nützlichkeitseinschätzung (Cappetta \& Paolino, 2015). Trainingsmotivation mediierte den Zusammenhang zwischen Trainingsgestaltung und Transfer. Diese Mediation wurde von Nützlichkeits- und Zufriedenheitseinschätzungen unmittelbar nach dem Training moderiert: Der Zusammenhang zwischen Trainingsmotivation und Transfer war größer, wenn Teilnehmende bessere Nützlichkeits- und Zufriedenheitseinschätzungen abgaben (Kodwani \& Prashar, 2019b).

Bezügliche Prädiktoren eines transferförderlichen Trainingsdesigns wurde ein reziproker Zusammenhang mit Transfermotivation bei zeitlich verteilten Trainings gefunden (Hinrichs, 2014). Zudem wurde ein Einfluss von Kollegenunterstützung, Vorgesetztenunterstützung und Anwendungsmöglichkeit auf die wahrgenommene transferförderliche Trainingsgestaltung aufgezeigt (Muduli \& Raval, 2018). 


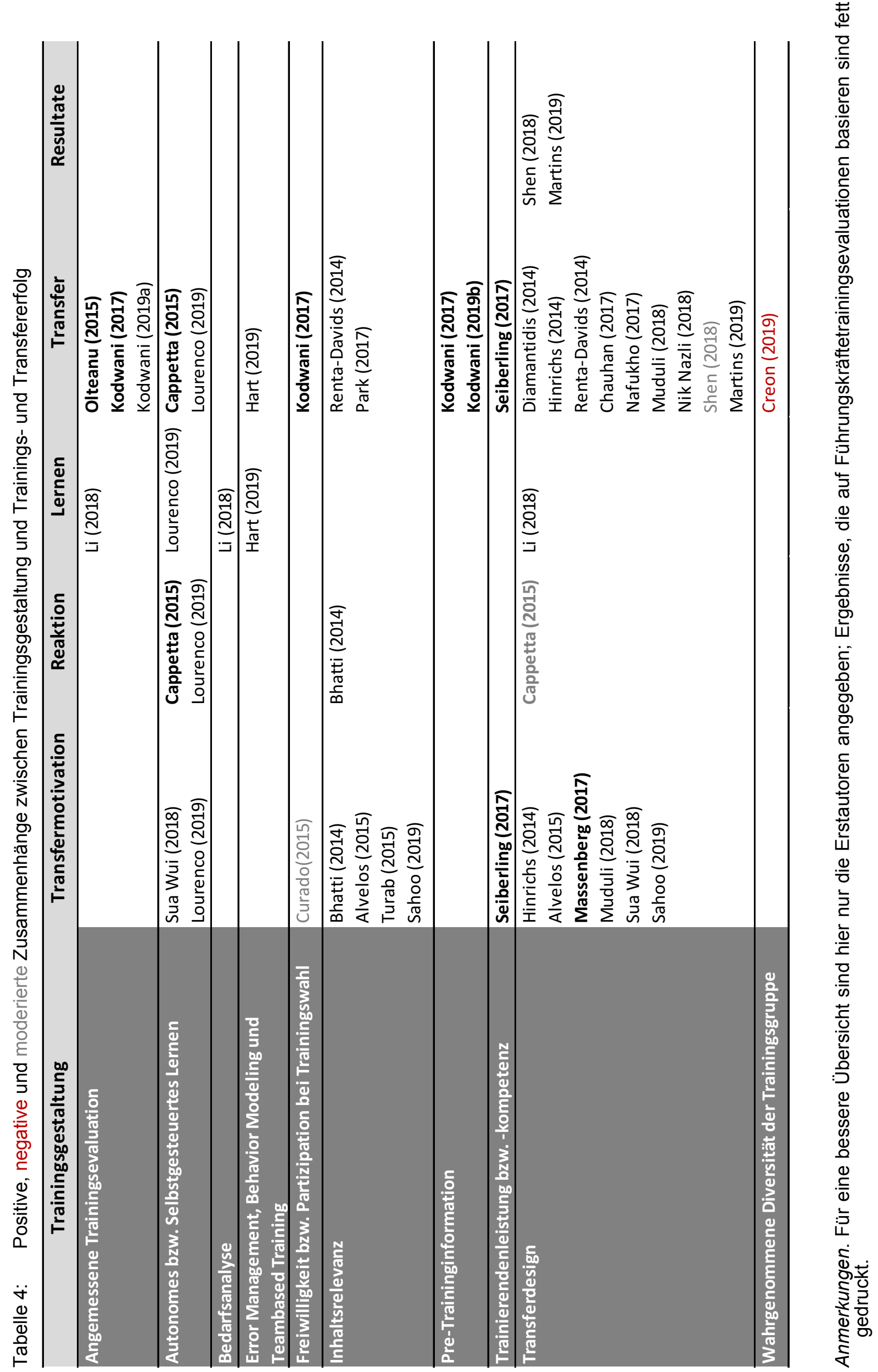




\subsubsection{Integrative Transfermodelle}

„Transfer can only be completely understood and predicted by examining the entire system of influences" (Holton et al., 2000, S. 336). Dieser Anforderung versuchen sogenannte integrative Transfermodell nachzukommen. Allerdings ist Trainingstransfer ein vielschichtiger Prozess, der von vielen Faktoren bestimmt wird, die eher simultan miteinander interagieren als lineare Zusammenhänge abzubilden (Y. Park et al., 2017). Somit ist eine ganzheitliche Abbildung des Transferprozesses eine komplexe Herausforderung, was bereits durch die zusammengefasste Befundlage prozessorientierter Transferforschung ersichtlich wurde. Nur durch sinnvolle Kategorisierung und Priorisierung relevanter Einflussfaktoren kann daher der Transferprozess in einem praktikablen Modell erklärt werden. Nach Gick und Holyoak (1987) lässt sich Transfer durch fünf Basiselemente erklären: Lernende, Lernaufgabe und -kontext sowie Transferaufgabe und -kontext. Diese von Piezzi (2002) als „Bestimmungsfaktoren“ von Transfer bezeichneten Einflussbereiche wurden in verschiedenen Transfermodellen präzisiert und deren Wirkzusammenhänge abgebildet. Transfermodelle, die die Trainingsforschung maßgeblich prägten, sind nachfolgend in chronologischer Folge zusammengefasst.

\section{Transferprozess von Noe und Schmitt (1986)}

Das älteste Transfermodell stammt von Noe und Schmitt (1986). Wie in Abbildung 8 ersichtlich, baut dieses Modell auf den vier Ebenen der Trainingswirksamkeit nach Kirkpatrick (1967, siehe auch Abschnitt 2.2 ab Seite 12) auf, ergänzt jedoch zentrale Wirkprozesse der Teilnehmendeneigenschaften und Arbeitsumgebung. Diese Erweiterung ist theoretisch aus dem Konstrukt der „trainability“ nach Wexley und Latham (1981) abgeleitet und um eine situative Komponente ergänzt. So wird Lern- und Transferleistung als eine Funktion aus Fähigkeit, Motivation und förderliche Arbeitsumgebung definiert. Wie diese Komponenten über den Trainings- und Transferprozess hinweg zusammenwirken ist als Transferprozess beschrieben. Demnach hängt Lernerfolg maßgeblich von Trainingsmotivation und Trainingsreaktionen (Zufriedenheits- und Nützlichkeitseinschätzung) ab. Lernerfolg führt wiederum erst in Kombination mit Transfermotivation zu Transfererfolg. Die Arbeitsumgebung wirkt durch transferförderliche Aufgabeninhalte und soziale Unterstützung auf den Prozess vor, während und nach Trainings ein. Sie fördert nicht nur die Trainings- und Transfermotivation, sondern bedingt auch (zusammen mit Arbeitseinstellungen), dass Transferverhalten langfristig in positiven Effekten resultiert. Zudem wird die Bedeutung der Feststellung des Kompetenzniveaus bzw. des Ausgangswertes der zu trainierenden Kompetenzen für passende Trainingsgestaltung und Trainingsmotivation erklärt. 


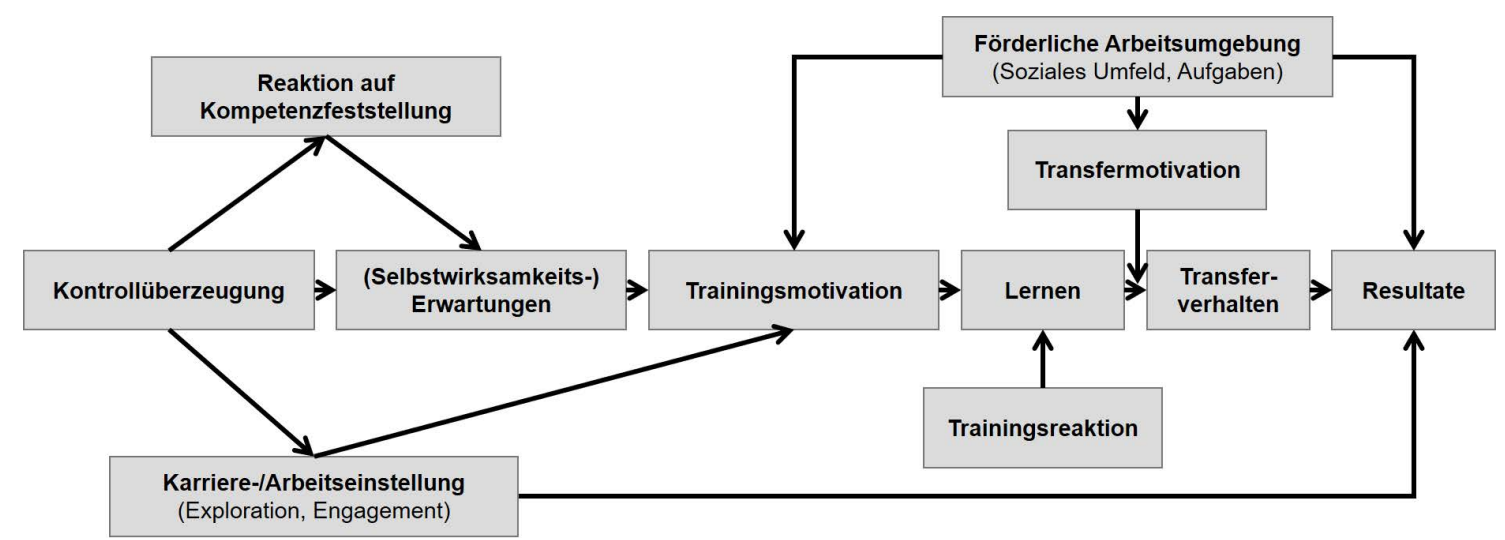

Abbildung 8: Transferprozess nach Noe und Schmitt (1986); eigene Übersetzung und Darstellung.

Die Grenzen des Modells bestehen insbesondere in der empirischen Überprüfung. Ergebnisse der Modellprüfung von Noe und Schmitt (1986) weisen zwar darauf hin, dass Engagement und Karriereabsicht relevante Prädiktoren zur Vorhersage für Kompetenzzuwachs und Verhaltensänderungen durch Trainings sind. Entgegen ihren Modellannahmen konnten sie jedoch den zentralen Einfluss der Transfermotivation inferenzstatistisch nicht nachweisen (was jedoch auch an der kleinen Stichprobe liegen kann). Obwohl die aktuelle Befundlage zentrale Modellannahmen des Transferprozesses bestätigt und das Modell bereits Lösungen für einige Kritikpunkte späterer Forschung bereitstellt, hat es sich nicht als Standardmodell in der Transferforschung durchgesetzt. Dennoch prägt es zentrale neuere Theorien (Cheng \& Ho, 2001; Holton, 2005; Quesada-Pallarès \& Gegenfurtner, 2015). Eine mögliche Erklärung könnte die Komplexität dieses umfassenden Modells mit konkreten Wirkzusammenhängen sein - was einerseits eine belastbare Überprüfung erschwert und andererseits auch für die Verwendung als strukturgebenden Rahmen für weitere Forschung nicht optimal geeignet ist. Zudem erfasst das Modell nur am Rande den Aspekt transferförderlicher Trainingsgestaltung. Diese Aspekte greift das etwas später veröffentlichte Modell von Baldwin und Ford (1988) auf, dessen Hauptkritik jedoch in der fehlenden Präzisierung der Wirkprozesse liegt. 


\section{Transfermodell von Baldwin und Ford (1988)}

Das einfachere und gleichzeitig umfassendere Transfermodell von Baldwin und Ford (1988) ist bis heute das einflussreichste Modell in der Transferforschung - obwohl es empirisch ebenfalls nicht ganzheitlich bestätigt ist. Die Autoren beabsichtigten bereits damals mit ihrem Review anhand einer ganzheitlichen Transfertheorie das „Transferproblem" zu lösen - ein Ziel, das bis heute nicht erreicht werden konnte. In ihrem Transfermodell, das in Abbildung 9 dargestellt ist, sind Transferfaktoren und -prozess zusammengefasst.

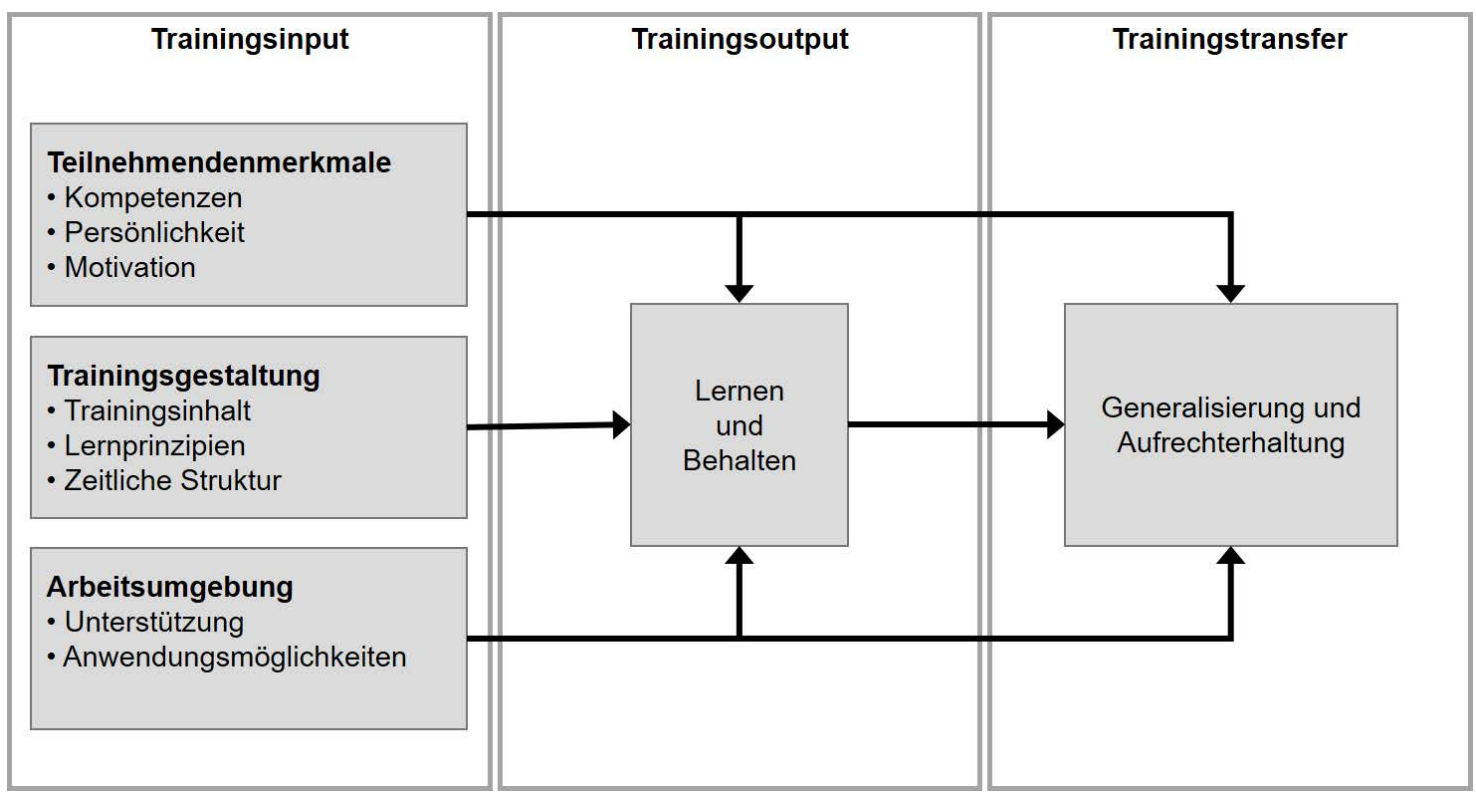

Abbildung 9: Transfermodell nach Baldwin und Ford (1988, S. 65); eigene Übersetzung und Darstellung.

Das Modell beschreibt den Transferprozess in drei Abschnitten: Trainingsinput, -output und -transfer. Dabei sind im Trainingsinput die relevanten Transferfaktoren enthalten, die auf drei Einflussbereiche des Transfers zurückgeführt werden können: Teilnehmendenmerkmale, Trainingsgestaltung und Arbeitsumgebung. Es wird angenommen, dass diese drei Trainingsinputfaktoren indirekt durch den Trainingsoutput bzw. die „Lernebene“ nach Kirkpatrick (1967, 1994) die Transferleistung beeinflussen. Darüber hinaus wird für Faktoren der Teilnehmendenmerkmale und Arbeitsumgebung zusätzlich ein direkter Transfereinfluss angenommen. Von Bedeutung ist auch die zweidimensionale Definition von Trainingstransfer (Generalisierung über verschiedene Kontextbedingungen hinweg und Aufrechterhaltung über die Zeit), die neben der übergeordneten Struktur die nachfolgende Transferforschung maßgeblich prägte. Insbesondere neuere Transferforschung betrachtet darüber hinaus auch Transferfaktoren der Teilnehmenden wie bei- 
spielsweise die Transfermotivation gleichzeitig als Trainingsoutput, da diese durch transferförderliche Trainingsgestaltung nachweislich erhöht werden kann (z. B. Massenberg et al., 2017; Sahoo \& Mishra, 2019).

Die Grenzen des Modells liegen insbesondere in der fehlenden Betrachtung von Wechselwirkungen zwischen den Inputfaktoren und deren Einflüsse über den Trainings- und Transferprozess hinweg. Zudem sind die Inputfaktoren nur sehr übergreifend beschrieben und wenig spezifiziert. Zwar zeigt das Modell auf an welchen Stellschrauben Transferförderung ansetzen kann, allerdings gibt es wenig konkrete Hinweise, wie transferförderliche Maßnahmen gestaltet werden sollten. Daher bietet das Modell zwar eine sehr wertvolle Rahmenstruktur zur Erklärung von Transfer, allerdings wenig konkrete Hinweise zu den Wirkmechanismen und der relativen und zeitlichen Bedeutung einzelner Transferdeterminanten. Entsprechend gibt es zahlreiche Versuche das Modell von Baldwin und Ford (1988) zu präzisieren (z. B. L. A. Burke \& Hutchins, 2008; Cannon-Bowers, Salas, Tannenbaum \& Mathieu, 1995; Dubs, 1990; Gegenfurtner et al., 2009; Hochholdinger et al., 2008; Piezzi, 2002; Rank \& Wakenhut, 1998; Seidel, 2012). Da jedoch bereits Baldwin und Ford (1988) bei ihrem Basismodell, das Problem zahlreicher zu berücksichtigenden Faktoren, ihrer Operationalisierung und zeitlichen Verortung thematisiert haben, sind diese erweiterten Modelle mit erhöhter Komplexität bei der Untersuchung des Lern- und Transferprozesses konfrontiert. Gleichzeitig werden immer wieder neue potenzielle Transferdeterminanten bzw. Wirkzusammenhänge zu unterschiedlichen Zeitpunkten und mit unterschiedlichen Erfolgskriterien identifiziert, weshalb auch diese umfangreicheren Modelle die Realität nicht umfassend abbilden können. So konnte sich bisher nur ein weiteres Transfermodell, das von Holton et al. (2000) entwickelte LTSI (siehe auch Abschnitt 2.3 ab Seite 15), in der Transferforschung etablieren, da es die Operationalisierung in Form eines global validierten Diagnoseinstruments mitliefert.

\section{Modell des Lerntransfer-System-Inventars von Holton et al. (2000)}

Das von Holton et al. (2000) entwickelte LTSI-Modell, resultiert aus einem mehrfach revidierten Evaluationsmodell (Holton, 1996, 2005; Holton \& Bates, 1997). Ausgangspunkt dabei war die Kritik an Kirkpatricks Modell $(1967,1998)$, das die von Noe und Schmitt (1986) aufgezeigte Bedeutung von Motivation und Arbeitsumgebung ausklammert und durch eine ergebnisorientierte Betrachtung keine Hinweise über gezielte Transferförderung geben kann. Holton (1996) schlug sein Modell daher als ganzheitlichere Alternative 
zu Kirkpatricks Evaluationsmodell vor ${ }^{18}$. Darin griff er einerseits die zentralen Bedeutung von Transfermotivation und transferförderlicher Arbeitsumgebung sowie die Funktion der „Trainability“ nach dem Transferprozessmodell von Noe und Schmitt (1986) auf. Andererseits integrierte er Ansätze des Transferklimamodells (insbesondere die Unterscheidung von sozialer Unterstützung und Transferfolgen) von Rouiller und Goldstein (1993) sowie nachfolgend empirisch überprüfte Faktoren zur Transferförderung. Das finale Evaluationsmodell (Holton, 2005) ist jedoch relativ komplex, was - wie der Autor selbst feststellt - eine empirische Überprüfungen erschwert. Als Lösung bietet er eine reduzierte Modellstruktur an, die auf der Validierung des LTSI basiert (Holton et al., 2000; revidierte Version Bates et al., 2012). Eine Version für den deutschsprachigen Raum, das sogenannte "German Learning Transfer System Inventory“ (GLTSI), wurde ebenfalls von Kauffeld et al. (2008) psychometrisch überprüft. Abbildung 10 zeigt das zugrundeliegende Modell. Grundsätzlich wird es von den Autoren für prozessorientierte Transferforschung im deutschsprachigen Raum empfohlen. Allerdings schlagen sie vor für den Einsatz des Fragebogens zwei Skalen noch weiter zu optimieren („Möglichkeit der Wissensanwendung“ und „Persönliche Transferkapazität“).

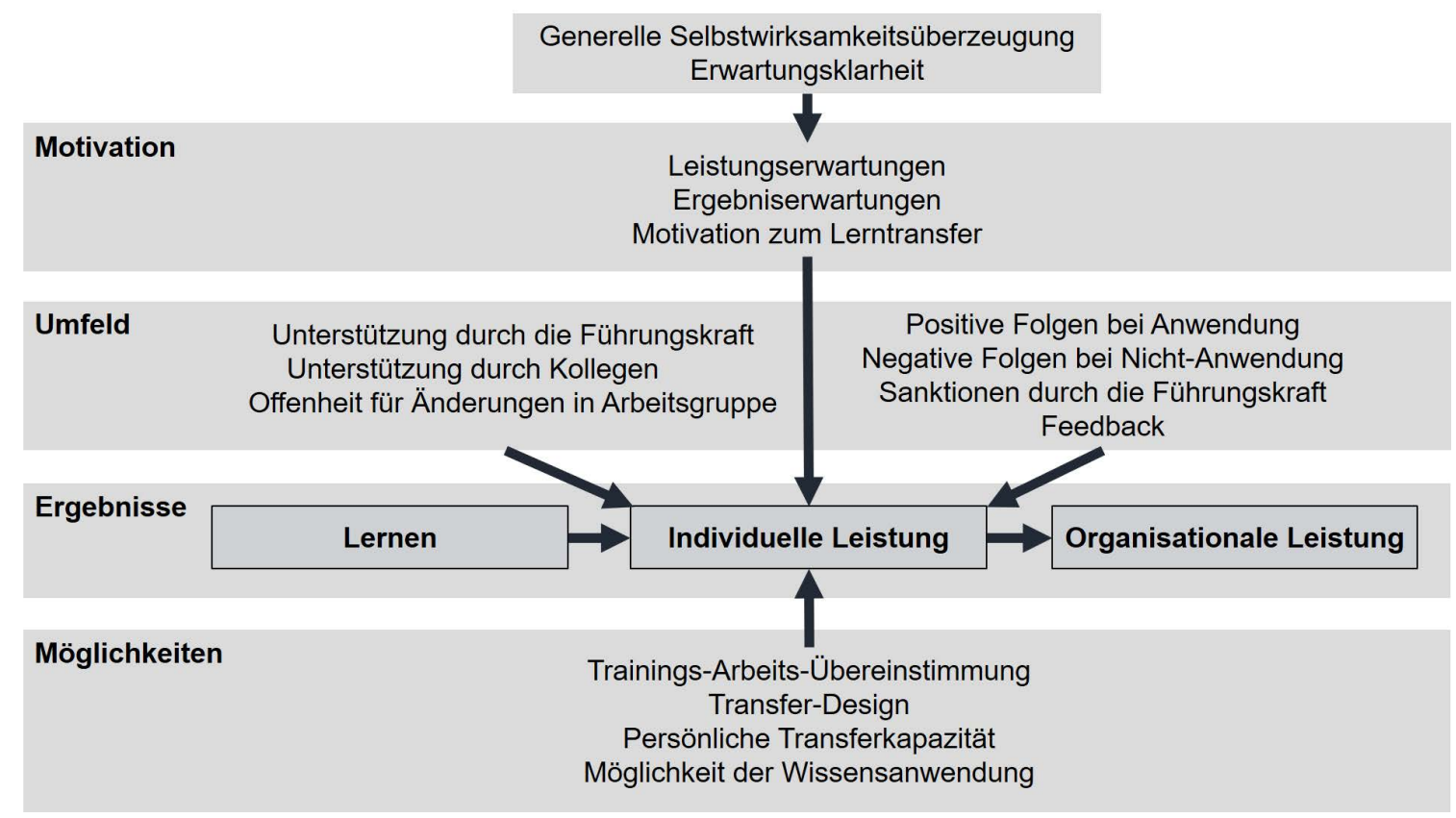

Abbildung 10: Modell des deutschen Lerntransfer-System-Inventars nach Kauffeld et al. (2008); eigene Darstellung.

18 Auch wenn das Modell von Kirkpatrick ursprünglich als Prozessmodell des Trainingserfolgs konzipiert war, liefert es zusätzlich zu Holtons Modell den Mehrwert einer hilfreichen Strukturierung des Transfererfolgs durch dessen vierdimensionale Definition. So werden in der vorliegenden Arbeit die Modelle von Holton (2005) und Kirkpatrick $(1967,1994)$ nicht als Alternativen, sondern als komplementäre Theorien betrachtet. 
Das LTSI-Modell umfasst 16 Transferfaktoren: fünf motivationale Faktoren (wovon zwei als distalere Vorläufer der übrigen drei definiert sind), sieben Faktoren transferförderlicher Arbeitsumgebung (die nach Transferunterstützung und -folgen unterschieden werden) und vier Faktoren der Möglichkeit zu Lernen beziehungsweise das Gelernte anzuwenden. Fünf dieser Transferfaktoren werden als relative stabil angenommen (Selbstwirksamkeitsüberzeugung, Leistungs- und Ergebniserwartungen sowie Offenheit der Arbeitsgruppe und Feedback), während die übrigen elf als trainingsspezifisch definiert sind. In der aktuellen Transferforschung, auch für Führungskräftetrainings, gilt das LTSIModell als „Best Practice“ Ansatz (Sørensen, 2017).

Grenzen des LTSI-Modells bestehen einerseits in der ganzheitlichen empirischen Bestätigung. Es ist beispielsweise bisher keine Studie bekannt, die zusammen mit den 16 operationalisierten LTSI-Faktoren auch den Einfluss des Lernerfolgs untersucht. Andererseits wird zwar ein ganzheitlicher Ansatz des LTSI-Modells postuliert, jedoch bestehen auch in diesem Modell Lücken: Bei der Fokussierung auf die Rolle der Transfermotivation, wird Lernerfolg als zentraler Transferfaktor etwas ausgeklammert. Er ist zwar im Modell, jedoch nicht in den operationalisierten Transferfaktoren des Fragebogens enthalten. Damit einhergehend wird zwar der „Trainability“-Ansatz von Noe und Schmitt (1986) explizit als ganzheitliches Konzept aufgegriffen (Holton et al., 2000), der „ability“Faktor wird jedoch auf situationale Ermöglichung bezogen und nicht wie in der Basisfunktion angenommen auf vorausgesetzte Fähigkeiten für Transfer, wie dem Lernerfolg. Zudem sind nicht alle empirisch bestätigten Transferfaktoren enthalten (siehe auch Sørensen, 2017). Beispielsweise sind Lern- und Trainingsmotivation oder die Ebene der Organisationsunterstützung nicht berücksichtigt, obwohl Metaanalysen ihre Bedeutung bestätigten (siehe Abschnitt 3.2.1 ab Seite 56). Dagegen zeigten Studien, die den kompletten LTSI einsetzten, dass nur ein Teil der Faktoren Transfereffekte hervorbringen ( $z$. B. Kauffeld et al., 2008; Zamani et al., 2016). Eine weitere Lücke besteht in der zeitlichen Komponente des Einflusses verschiedener Transferfaktoren und ihrer Wirkzusammenhänge untereinander. Es sind vorwiegend direkte Transfereinflüsse im LTSI-Modell abgebildet, obwohl die Modelle von Noe und Schmitt (1986) und Baldwin und Ford (1988) sowie die nachfolgende Transferforschung für einige der erfassten Transferfaktoren vorwiegend indirekte Transferwirkungen nahelegt (z. B. Reinhold et al., 2018; Renta-Davids et al., 2014; Zamani et al., 2016). Eine Integration in das Modell von Baldwin und Ford (1988) und eine stärkere Differenzierung zwischen vorwiegend direkten und indirekten Transferfaktoren könnte den Anspruch auf Ganzheitlichkeit mit der notwendigen Komplexitätsreduktion verbinden. 


\subsubsection{Zusammenfassung prozessorientierter Trainingsforschung}

Studien prozessorientierter Transferforschung haben über die vergangenen Jahrzehnte hinweg ein beeindruckendes Volumen erreicht und umfassende Erkenntnisse zu Ergründung von Transferdeterminanten und -prozessen geliefert. In regelmäßigen Reviews, Metaanalysen und integrativen Transfermodellen wurde die Befundlage systematisch zusammengefasst. Allerdings sind diese Zusammenfassungen für ganzheitliche Überprüfungen sowie für die Trainingspraxis sehr komplex und können die Realität dennoch nicht vollständig abbilden. Alle integrativen Transfermodelle zeichnen sich daher dadurch aus, dass zwar die einzelnen Transferfaktoren durch empirische Untersuchungen von Teilzusammenhängen als bestätigt gelten können. Ganzheitliche Überprüfungen mit den angenommenen Interaktionen zur Bestätigung der Modelle stehen allerdings noch aus, da es methodisch schwer möglich ist alle transferrelevanten Faktoren mit ihrem komplexen Beziehungsgefüge zu erfassen. So hat sich die Transferforschung in gewisser Weise in ihrer eigenen Komplexität verfangen, was von Baldwin et al. (2017) als „Bricklayer-Phänomen“ bezeichnet wurde. Die Autoren diagnostizierten eine Fixierung auf die Gewinnung von Wissensausschnitten, sogenannten „Bricks“, wobei dem zentralen Wirkmechanismus der Teilnehmendenmerkmale, der den eigentlichen Transferprozess darstellt, eine unzureichende Berücksichtigung zukommt. Um dieses umfassende Wissen jedoch auch für die Lösung des Transferproblems nutzbar zu machen besteht die zentrale Herausforderung der Transferforschung weniger in der weiteren Überprüfung einzelner Wirkzusammenhänge, sondern viel mehr in einer ganzheitlichen und praktikablen Konsolidierung der reichhaltigen Erkenntnisse. Dafür empfahlen die Autoren eine teilnehmendenzentrierte Perspektive zur Erklärung des Transferprozesses einzunehmen und Transferfaktoren nach ihrem Einfluss zu priorisieren, um daraus einen gezielten Ressourceneinsatz für Transfermanagementmaßnahmen abzuleiten.

Daher wird hier ein Ansatz vorgeschlagen, um die bisherige prozessorientierte Trainingsforschung in diesem Sinne zusammen zu fassen und zentrale Wirkmechanismen abzuleiten. Wie in Abschnitt 3.2.2 ab Seite 70 aufgezeigt, ist das Transfermodell von Baldwin und Ford (1988) das bewährteste Modell zur Strukturierung prozessorientierter Trainingsforschung. Das LTSI-Modell von Holton et al. (2000) gilt dagegen als „Best Practice“ zur umfassenden Ergründung des Transferprozesses. Daher werden diese beiden Modelle teilnehmendenzentriert integriert und um aktuelle Befunde (siehe Abschnitt 3.2.1 ab Seite 45) ergänzt. In einem weiteren Schritt wird - wie bereits von Noe und Schmitt (1986) vorgeschlagen - die Formel individueller Leistung auf Transferleistung übertragen, um zwischen vorwiegend direkten und indirekten Transferdeterminanten zu unterscheiden. Allerdings wird die situative Komponente auf Anwendungsmög- 
lichkeiten als direkter Prädiktor bezogen, da nach aktueller Befundlage Transferunterstützung und -konsequenzen als vorwiegend indirekt wirkende Faktoren gelten. Daraus resultiert die in Abbildung 11 dargestellte Zusammenfassung zentraler Wirkmechanismen des Trainingstransfers.

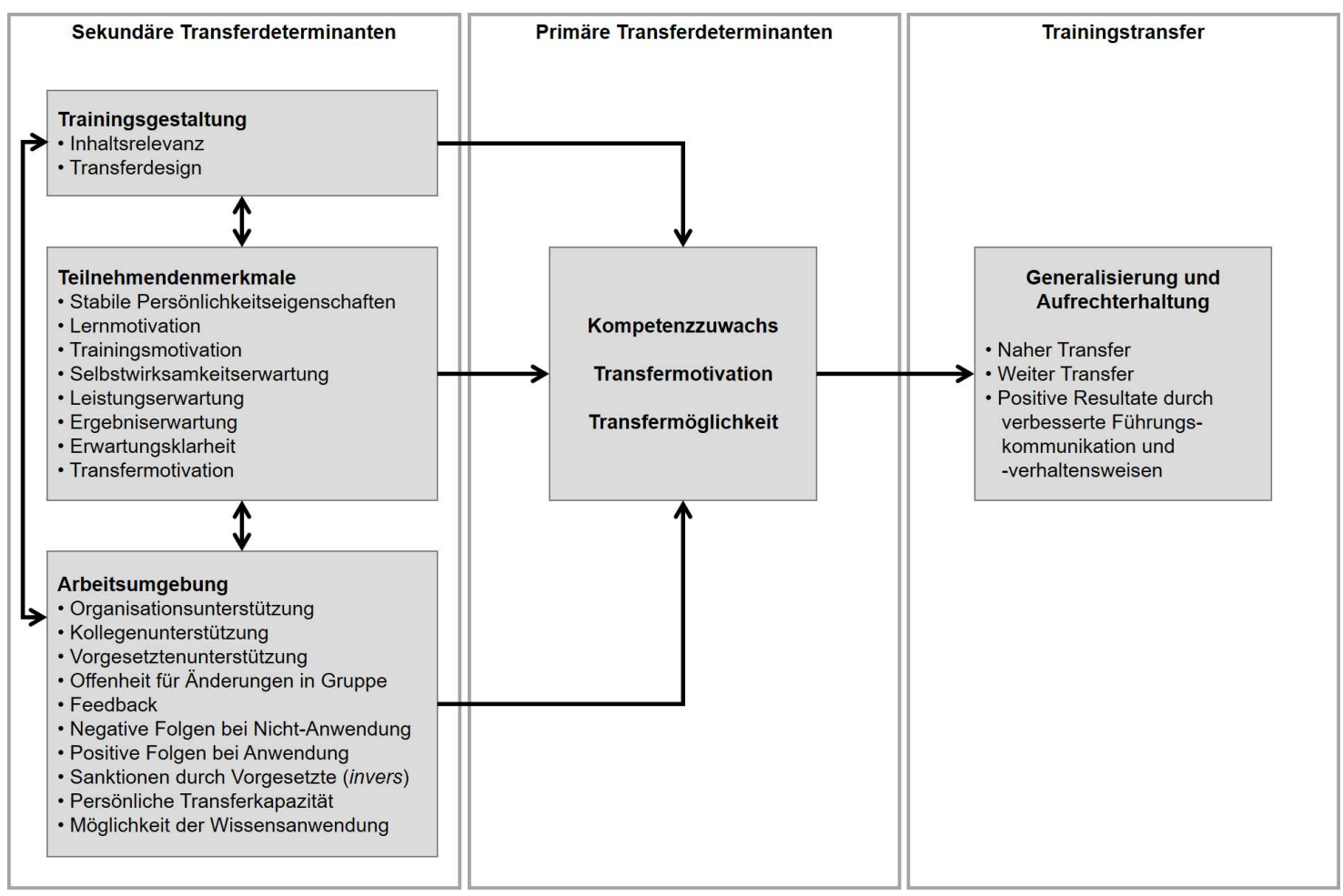

Abbildung 11:Zusammenfassung zentraler Wirkmechanismen des Trainingstransfers; eigene Darstellung.

Demnach wird Transfer als direkte Funktion von Kompetenzzuwachs, Transfermotivation und -möglichkeit definiert, die daher als primäre Transferdeterminanten bezeichnet werden. Dabei werden reziproke Zusammenhänge zwischen diesen drei direkten Prädiktoren gemäß etablierter Motivationstheorien angenommen (beispielsweise steigert Transfermotivation auch Bemühungen zu Lernen oder Anwendungsmöglichkeiten zu finden, Lernerfolg und erwartete Anwendungsmöglichkeit wirken wiederum motivationsförderlich). Zudem wird von einer multiplikativen Verknüpfung ausgegangen, da von allen drei Komponenten ein Mindestmaß vorhanden sein muss, damit Transfer zustande kommt. Dennoch kann angenommen werden, dass sie sich auch teilweise kompensieren können (Iddekinge et al., 2018). Im Idealfall sollte sich diese Transferfunktion über die Transferzeit hinweg selbstverstärken. Indem erstes Transferverhalten in positiven Transferkonsequenzen resultiert, werden Kompetenz, Motivation und Möglichkeiten für 
weiteren Transfer verstärkt bzw. begünstigt, wodurch Transfer nachhaltig aufrechterhalten oder sogar gesteigert wird. Allerdings kann diese Funktion auch in einem Negativkreislauf enden (siehe auch Blume et al., 2019).

Entsprechend werden die übrigen transferförderlichen Teilnehmendenmerkmale des LTSI (Erwartungsklarheit, Selbstwirksamkeit, Leistungs- und Ergebniserwartungen) sowie die weiteren fundierten Faktoren (transferrelevante Persönlichkeitseigenschaften: Gewissenhaftigkeit und Emotionale Stabilität sowie kognitive Fähigkeiten bei ClosedSkills-Trainings, Lern- und Trainingsmotivation) als vorwiegend indirekte bzw. sekundäre Transferdeterminanten erachtet. Zusammen mit den sekundären Transferdeterminanten der Trainingsgestaltung (durch Bedarfsanalysen gesicherte Inhaltsrelevanz, fundierte Lehrmethoden sowie zeitlich verteiltes Training mit Pre- und Post-Traininginterventionen) und Arbeitsumgebung (Transferunterstützung und -konsequenzen sowie erwartete Anwendungsmöglichkeiten) wirken diese vor, während und nach Trainings auf die primären Transferdeterminanten ein. Wie die Befunde aufzeigen, sind auch die sekundären Transferfaktoren miteinander verknüpft und können sich teilweise kompensieren. So begünstigen beispielsweise Lernmotivation und Selbstwirksamkeit die Trainingsmotivation. Die Trainingsmotivation hat wiederum einen Einfluss auf die Trainingsreaktion bezüglich Bewertung von Inhaltsrelevanz und Nützlichkeit des Trainings. Eine transferförderliche Trainingsgestaltung sollte sich nicht nur auf die transferrelevanten Teilnehmendenmerkmale auswirken, sondern auch auf die Transferdeterminanten der Arbeitsumgebung. Insbesondere können und sollten erwartete Anwendungsmöglichkeiten und Transferunterstützung durch Trainings begünstigt werden.

Allerdings muss berücksichtigt werden, dass die Studienbasis für diese Zusammenfassung zentraler Transfermechanismen nicht spezifisch auf Führungskräftetrainings ausgerichtet ist, da es zu wenige Evaluationsstudien von Führungskräftetrainings für belastbare Aussagen gibt. Zudem resultieren die Befunde vorwiegend aus retrospektiven Eingruppen-Querschnittstudien, die auf Selbsteinschätzung per Fragebogen ohne Kontrolle von Ausgangswerten, Trainings- oder Organisationssettings basieren. Dadurch werden Verzerrungen beispielsweise aufgrund konfundierender Einflüsse, sozialer Erwünschtheit und Same-Source-Same-Measurement-Kontext begünstigt (Podsakoff, MacKenzie \& Podsakoff, 2012; Scriven, 1991). Querschnittstudien können den Transferprozess nicht ausreichend erforschen, da Transferfaktoren vor und nach Trainings spezifische Wirkungen zeigen, wobei zu unterschiedlichen Zeitpunkten jeweils andere Faktoren von Bedeutung sind. Insbesondere die Ausprägung von Transferfaktoren vor Trainings und Ausgangswerte der Trainingsziele werden nur selten berücksichtigt (siehe auch L. A. Burke \& Hutchins, 2007; Massenberg et al., 2017). Zudem werden in bisheriger Trans- 
ferforschung nur Ausschnitte des Transferprozesses, statt ganzheitliche Modelle überprüft. So gibt es kaum systematische Untersuchungen der Wechselwirkungen zwischen den identifizierten Transferfaktoren. Es ist der Autorin auch keine Studie bekannt, die die drei zentralen Prädiktoren Kompetenzzuwachs, Transfermotivation und -möglichkeit in einem Modell überprüft. Auch die einzelnen Einflüsse von Lernen und Anwendungsmöglichkeit werden im Vergleich zur Transfermotivation relativ selten betrachtet. Studien, die Einflüsse relevanter Transferfaktoren in einem ganzheitlichen Modell und zu unterschiedlichen Messzeitpunkten im Transferprozess erfassen, stellen daher trotz der umfassenden Forschungsaktivitäten eine Forschungslücke dar. Darüber hinaus zeichnet sich die prozessorientierte Transferforschung durch wenig Informationen zu Trainingsinhalt und -methoden sowie uneinheitliche und teilweise nicht klar angegebene Definitionen der analysierten Variablen aus. Insbesondere in Bezug auf Transferkriterien scheint es jedoch wichtig zu sein eine klare Spezifizierung vorzunehmen. Wie bereits von (Laker, 1990) aufgezeigt, empfiehlt sich dabei eine Differenzierung von 1) nahem und weitem Transfer nach dem Grad der Ähnlichkeit zwischen Lern- und Funktionsfeld (siehe auch Abschnitt 2.3 ab Seite 15) und 2) nach der zeitlichen Dimension in erstes Transferverhalten und Aufrechterhaltung von nachhaltigem Transfererfolg (siehe auch Ford et al., 2018). Arthur et al. (2003) empfahlen basierend auf ihrer Metaanalyse für die Überprüfung von aufrechterhaltenem Transferverhalten eine Transferzeit bzw. einen Zeitraum der Wirksamkeitsprüfung von mindestens 6 Monaten nach dem Training zu wählen. Die meisten der wenigen Studien, die eine Aufrechterhaltung über 6 Monate nach Trainings überprüfen, fassen jedoch Transfermessungen zusammen, die sich in ihrer Transferzeit um mehrere Jahre unterscheiden (z. B. Chauhan et al., 2017; Diamantidis \& Chatzoglou, 2014; Grohmann et al., 2014; Homklin et al., 2014; Massenberg \& Kauffeld, 2015; Miiro et al., 2014). Zudem geben viele Studien gar keine Information über die betrachtete Transferzeit an (z. B. Nik Nazli \& Sheikh Khairudin, 2018; S. Park et al., 2018).

Zusammengefasst liefert die prozessorientierte Transferforschung bereits reichhaltige Erkenntnisse zentraler Wirkmechanismen des Trainingstransfers. Allerdings gilt es diese ganzheitlich und praktikabel zu integrieren und in möglichst kontrolliertem Rahmen zu überprüfen, um daraus nutzbare Lösungen des Transferproblems ableiten zu können. Dabei ist es wichtig, sowohl die Intervention als auch den Trainings- und Transfererfolg klar zu spezifizieren, da je nach Transferkriterium oder Trainingsinhalt unterschiedliche Zusammenhänge identifiziert wurden (insbesondere für Trainings interaktionaler Kompetenzen zeigt die Befundlage spezifische Wirkzusammenhänge). Daher bezieht sich die vorliegende Arbeit spezifisch auf die Überprüfung der nachhaltigen Aufrechterhaltung von nahem und weitem Transfer nach Führungskräftetrainings zur Vermittlung von interaktionalen Kompetenzen, da dieser Fokus für die Personalentwicklungspraxis von 
größter Relevanz ist. Einerseits, weil diese Trainingsform die meist genutzte und institutionalisierte Personalentwicklungsmaßnahme mit einem potenziell weiten Wirkradius darstellt (z. B. Felfe \& Franke, 2014; Martin et al., 2017). Andererseits, weil bei diesem Fokus das Transferproblem besonders ausgeprägt ist, da eine sehr große Distanz zwischen Lern- und Anwendungsfeld liegt (z. B. Laker \& Powell, 2011; Poell, 2017).

Allerdings besteht das Transferproblem in einem doppelten Sinn nicht nur aufgrund von Forschungslücken, sondern auch aufgrund mangelnden Forschungstransfers in die Praxis. Trotz der erkannten Relevanz und den zahlreichen Handlungsimplikationen, Checklisten und Diagnoseinstrumenten aus der prozessorientierten Forschung, finden kaum fundierte Transfermanagementaktivitäten in der Trainingspraxis statt (Diesner \& Seufert, 2013; Fandel-Meyer et al., 2015; Weinbauer-Heidel, 2016). Daher wird in einem dritten Abschnitt des Literaturreviews der aktuelle Forschungsstand zum Research-PracticeGap der Transferforschung zusammengefasst. 


\subsection{Research-Practice-Gap der Transferforschung}

Obwohl Manager und Bildungsverantwortliche von Unternehmen anhaltend mit dem Wirkungsgrad von Personalentwicklungsmaßnahmen unzufrieden sind (Beer, Finnstrom \& Schrader, 2016) und insbesondere die Wirksamkeit von klassischen formalen „off-thejob“ Führungskräftetrainings zur Vermittlung von Softskills in Frage gestellt werden (Kaiser \& Curphy, 2013; Laker \& Powell, 2011; Poell, 2017; J. Schwartz et al., 2014; Staudt \& Kriegesmann, 1999), finden kaum fundierte Transfermanagementaktivitäten in der Trainingspraxis statt (L. A. Burke \& Hutchins, 2007; Diesner \& Seufert, 2013; FandelMeyer et al., 2015; Salas \& Kosarzycki, 2003; Salas et al., 2012; Weinbauer-Heidel, 2016).

Autoren, die diesen Research-Practice-Gap thematisieren, erklären die fehlende Beachtung und Nutzung der Transferforschung insbesondere durch drei Gründe: 1) Unzureichende Praxis- bzw. Aktionsorientierung der Forschungsbefunde: Komplexe und unterschiedliche Transfer- bzw. Evaluationsmodelle verwirren eher als praktikable Transfermanagementansätze aufzuzeigen (Baldwin et al., 2017; Sahoo \& Mishra, 2017; Salas \& Kosarzycki, 2003). Denn „[...] most existing authors have stopped at the point of identifying, describing, or measuring factors that may influence transfer without investigating how those factors might be effectively changed or managed" (Cheng \& Hampson, 2008, S. 331). 2) Das weitverbreitete Misskonzept basierend auf einer simplifizierten Betrachtung von Trainings, dass "[...] managerial behaviour [would] automatically change following formal training and development activities without the need to actively support the process" (Johnson, Blackman \& Buick, 2018, S. 383). 3) Befunde der Transferforschung in psychologischen Fachzeitschriften sind für Personalverantwortliche nicht zugänglich bzw. werden nicht als Quelle für Handlungsimplikationen genutzt $(H$. M. Hutchins et al., 2010).

Weinbauer-Heidel (2016) hat den Research-Practice-Gap der Transferforschung für österreichische Unternehmen überprüft und darüber hinaus folgende hemmende Faktoren anhand einer Delphi-Studie identifiziert:

- Fehlendes Wissen über Transferforschung durch Bildungshintergrund (Lücke von Studiengängen, Aus- und Fortbildungen; z. B. ist Transferforschung nicht in klassischen Personalmanagementstudiengängen enthalten)

- Fehlende(s) Zuständigkeitsgefühl bzw. Transferverantwortung aller beteiligten Akteure

- Ressourcenmangel

- Gewohnheit (Training ohne Transfersicherung ist institutionalisiert) 
- Fehlende Dringlichkeit

- Fehlende Messung des Transferwirksamkeit

- Angst vor Offenlegung des Transferproblems

- Trainerauswahl- bzw. Trainingserfolgskriterien

(eher Sympathie und Zufriedenheit als Evidenzbasierung und Nützlichkeit)

- Zweifel an der Wirksamkeit von Transfermanagementmaßnahmen und deren sinnvollen Überprüfbarkeit

Dabei kann angenommen werden, dass insbesondere der letzte Aspekt bezüglich Zweifel an der Wirksamkeit von Transfermanagementmaßnahmen, die übrigen hemmenden Faktoren begünstigt. Insgesamt weisen die Ergebnisse der Delphi-Studie darauf hin, dass Personalverantwortliche die Transferforschung nicht als praxisrelevante Erkenntnisquelle betrachten. Stattdessen werden Handlungsempfehlungen für Transfermanagement vorwiegend aus informellem Erfahrungsaustausch genutzt. Es wird aber auch der Mangel eines wissenschaftlich fundierten und anerkannten Leitfadens für die Implementierung eines systematischen Transfermanagements als zentrale Lücke wahrgenommen. Aufgrund der räumlichen, sprachlichen, kulturellen und bildungsorientierten Nähe, kann angenommen werden, dass diese Faktoren auch auf deutsche Unternehmen übertragbar sind.

Eine Untersuchung zum Weiterbildungsverhalten von Personalverantwortliche bestätigte einige Aspekte für den deutschen Raum, indem aufgezeigt wurde, dass neben Weiterbildungen insbesondere deutschsprachige Print- und Onlinemedien als Quelle für Lösungen von neuen Herausforderungen genutzt werden. Personalverantwortliche mit Psychologiestudium nutzten am ehesten (wissenschaftliche) Fachzeitschriften, wobei auch diese Berufsgruppe deutschsprachige Publikationen präferierte (Kanning \& Thielsch, 2015). Die Autoren leiteten zudem folgende Handlungsimplikationen zur Reduktion von Research-Practice-Gaps ab: Wissenschaftliche Artikel müssen 1) aktuelle Praxisthemen aufgreifen, 2) ihren Praxisbezug klar hervorheben, 3) deutschsprachig und 4) leicht verfügbar publiziert sein.

Insgesamt kann aus den Befunden zum Research-Practice-Gap resümiert werden, dass zukünftige Transferforschung stärker praxisorientiert zusammengefasst und publiziert werden sollte. Dabei gilt es die Wirksamkeit von empfohlenen Transfermanagementmaßnahmen aufzuzeigen und Implementierungsmöglichkeiten in Leitfäden zusammenzufassen. 


\subsection{Kapitelzusammenfassung}

Ergebnisorientierte Forschungsbefunde zeigen, dass Führungskräftetrainings nachhaltige Effekte auf allen vier Ebenen der Trainingswirksamkeit nach Kirkpatrick (1967, 1998) hervorbringen können. Insgesamt weisen aktuelle Befunde darauf hin, dass formale Führungskräftetrainings wesentlich effektiver sind als ihr Ruf (Lacerenza et al., 2017; Martin et al., 2017; Sørensen, 2017). Allerdings gibt es abhängig von Trainingsdesign und Evaluation große Unterschiede der Wirksamkeit. Metaanalytisch wird aufgezeigt, dass evidenzbasierte und angemessen evaluierte Trainings für einen nachhaltigen Wirkungsgrad auf allen Ebenen der Trainingswirksamkeit entscheidend sind. Dabei scheinen insbesondere Bedarfsanalysenbasierung, fundierte Kombination verschiedener Trainingsmethoden, Feedback und zeitlich verteiltes Präsenzformat wichtig zu sein. Allerdings stehen umfassende Überprüfungen im Rahmen ganzheitlicher Evaluationsstudien, die Trainingswirksamkeit auf allen vier Dimensionen betrachten noch aus. Eine Kombination aus Kontrollgruppenvergleichen und Mehrfachmessung mit angemessenem zeitlichem Abstand zum Training sowie die Nutzung weiterer Datenquellen in Ergänzung zur reinen Selbsteinschätzung (insbesondere für die Resultatebene) sind anhaltende Forschungslücken. Dabei ist auch eine aussagekräftige Dokumentation relevanter Eigenschaften von Intervention und Evaluation wichtig. Darüber hinaus ist eine reine ergebnisorientierte Betrachtung auf der Makroebene für die Lösung des Transferproblems unzureichend. Um die Frage zu beantworten, von welchen Faktoren und Maßnahmen die Trainingswirksamkeit abhängt und wie diese gezielt gefördert werden kann, muss eine prozessorientierte Evaluationsperspektive zur Überprüfung individueller Transferleistung integriert werden (siehe auch Gessler, 2012).

Prozessorientierte Forschungsbefunde zeigen dafür zahlreiche potenziell relevante Transferdeterminanten auf, die im integrativen LTSI-Modell zusammengefasst sind. Anhaltspunkte zu den komplexen Wirkzusammenhängen der Transferdeterminanten können insbesondere von aktuellen Befunden und deren teilnehmendenzentrierten Integration in das Transfermodell von Baldwin und Ford (1988) abgeleitet werden. Durch eine stärkere Differenzierung zwischen vorwiegend direkten und indirekten Transferdeterminanten kann Ganzheitlichkeit mit der notwendigen Komplexitätsreduktion verknüpft werden. Eine Fortführung zentraler Annahmen des Transferprozessmodells von Noe und Schmitt (1986) durch die aktuelle Befundlage ermöglicht dafür eine theoretisch fundierte Priorisierung der relativen Einflüsse der zahlreichen Transferdeterminanten. Demnach kann angenommen werden, dass Transferleistung eine direkte Funktion von Kompetenzzuwachs, Transfermotivation und -möglichkeit ist, während die Wirkung der übrigen 
Transferdeterminanten durch diese drei Prädiktoren vermittelt wird. Allerdings steht eine Bestätigung dieser Annahme bzw. Priorisierung noch aus.

Wie aktuelle Forschungsarbeiten zum Research-Practice-Gap der Transferforschung aufzeigen, scheint die ganzheitliche ergebnisorientierte Überprüfung fundierter Transfermanagementaktivitäten sowie ein daraus abgeleiteter fundierter und praxisorientierter Leitfaden entscheidend zu sein. Nur so kommen wissenschaftliche Erkenntnisse für die Lösung des Transferproblems in der Personalentwicklungspraxis an. 


\section{$4 \quad$ Forschungsgegenstand und konkrete Forschungsfragen}

"There is potential for a much greater yield on the enormous organizational investments in training - but only if researchers are conducting the types of studies and synthesizing the knowledge learned in ways that stimulate thinking about transfer and ultimately are meaningful to professionals" (Ford et al., 2018).

\subsection{Ableitung von Hypothesen und Überprüfungsmethoden}

Die aktuelle Forschungslage zur Effektivität von Führungskräfteentwicklung legt nahe, dass Trainings generell nachhaltige Effekte erzielen können. Dabei kann die Berücksichtigung verschiedener Transferfaktoren nachweislich die Trainingswirksamkeit steigern (siehe Abschnitt 3.1 ab Seite 31). Die spezifischere Forschung zum Trainingstransfer konzentriert sich auf die Ergründung relevanter Transferfaktoren und betont deren Bedeutung für den Trainingswirkungsgrad (siehe Abschnitt 3.2 ab Seite 43). Obwohl es inzwischen zahlreiche Befunde und Empfehlungen für transferförderliche Trainingsgestaltung auch im Kontext deutscher Führungskräfteentwicklung gibt (Felfe \& Franke, 2014; Hochholdinger et al., 2008; Kauffeld, 2016; Solga, 2011b), werden sie in der gängigen Trainingspraxis kaum umgesetzt (Salas \& Kosarzycki, 2003; Salas et al., 2012; Tonhäuser \& Bücker, 2016; Wang \& Wilcox, 2014). Ob das bestehende Transferproblem an mangelnder Praktikabilität und Effektivität der Handlungsimplikationen bisheriger Transferforschung liegt, soll durch das vorliegende Forschungsprojekt mit dem Fokus auf Führungskräftetrainings anhand folgender Forschungsfragen überprüft werden.

1. Forschungsfrage (ergebnisorientiert): Lassen sich die Befunde und

Empfehlungen für transferförderliche Trainingsgestaltung in

Führungskräftetrainings wirksam umsetzten?

Hypothese 1: Ein transferförderlich gestaltetes Training ist wirksam (a) bezüglich kurzfristigem Trainingserfolg durch positive Trainingsreaktion

(Nützlichkeitseinschätzung und Transfermotivation) und Kompetenzentwicklung sowie (b) bezüglich langfristigem Transfererfolg durch Transferverhalten (naher und weiter Transfer) und dessen Resultaten (Verbesserung in Führungskommunikation und -verhalten).

2. Forschungsfrage (prozessorientiert): Wie hängen die Wirkmechanismen des Trainingstransfers bei Führungskräftetrainings zusammen, welche Transferdeterminanten sind für individuelle Transferleistung von besonderer Bedeutung und können diese durch transferförderliche Trainingsgestaltung vorhergesagt werden? 
Hypothese 2: Transfer kann durch (a) Kompetenz, (b) Transfermotivation und (c) -möglichkeit maßgeblich vorhergesagt werden, (d) wobei Kompetenz den größten Einfluss zeigt.

Hypothese 3: Kompetenz, Transfermotivation und -möglichkeit können durch transferförderliche (a) Teilnehmendenmerkmale, (b) Arbeitsumgebung und (c) Trainingsgestaltung maßgeblich vorhergesagt werden, (d) wobei Trainingsgestaltung den größten Einfluss zeigt.

Das zur Hypothesenprüfung genutzte Transfermodell ist in Abbildung 12 dargestellt. Dabei sind die im LTSI-Modell von Holton et al. (2000) bzw. in dessen deutscher Übersetzung von Kauffeld et al. (2008) zusammengefassten Transferdeterminanten in das Basismodell des Trainingstransfers von Baldwin und Ford (1988) integriert. Auch wenn das LTSI-Modell noch nicht ganzheitlich überprüft wurde (Kauffeld et al., 2008; Sørensen, 2017), bietet es für das vorliegende Forschungsprojekt eine sehr nützliche Struktur für eine umfassende und dennoch ökonomische Erfassung relevanter Transferfaktoren. Basierend auf zusätzlichen Befunden wird es jedoch durch weitere Teilnehmendenmerkmale ergänzt: Lernorientierung und Trainingsmotivation nach Noe und Wilk (1993). Eine Erfassung stabiler Persönlichkeitseigenschaften wie Gewissenhaftigkeit und emotionale Stabilität ${ }^{19}$, wird - trotz ihres bestätigten Einflusses auf Transfer - für die Trainingspraxis als ungeeignet erachtet (insbesondere aufgrund der Sensibilität gegenüber Persönlichkeitstests). Zudem wird Organisationsunterstützung nicht erfasst, da das organisationale Setting zur Reduktion konfundierender Einflüsse konstant gehalten wird.

${ }^{19}$ Kognitive Fähigkeiten sind bei Führungskräftetrainings interaktionaler Kompetenzen (Blume et al., 2010) und Überprüfung typischer (vs. maximaler) Transferleistung weniger relevant (Huang et al., 2015). 


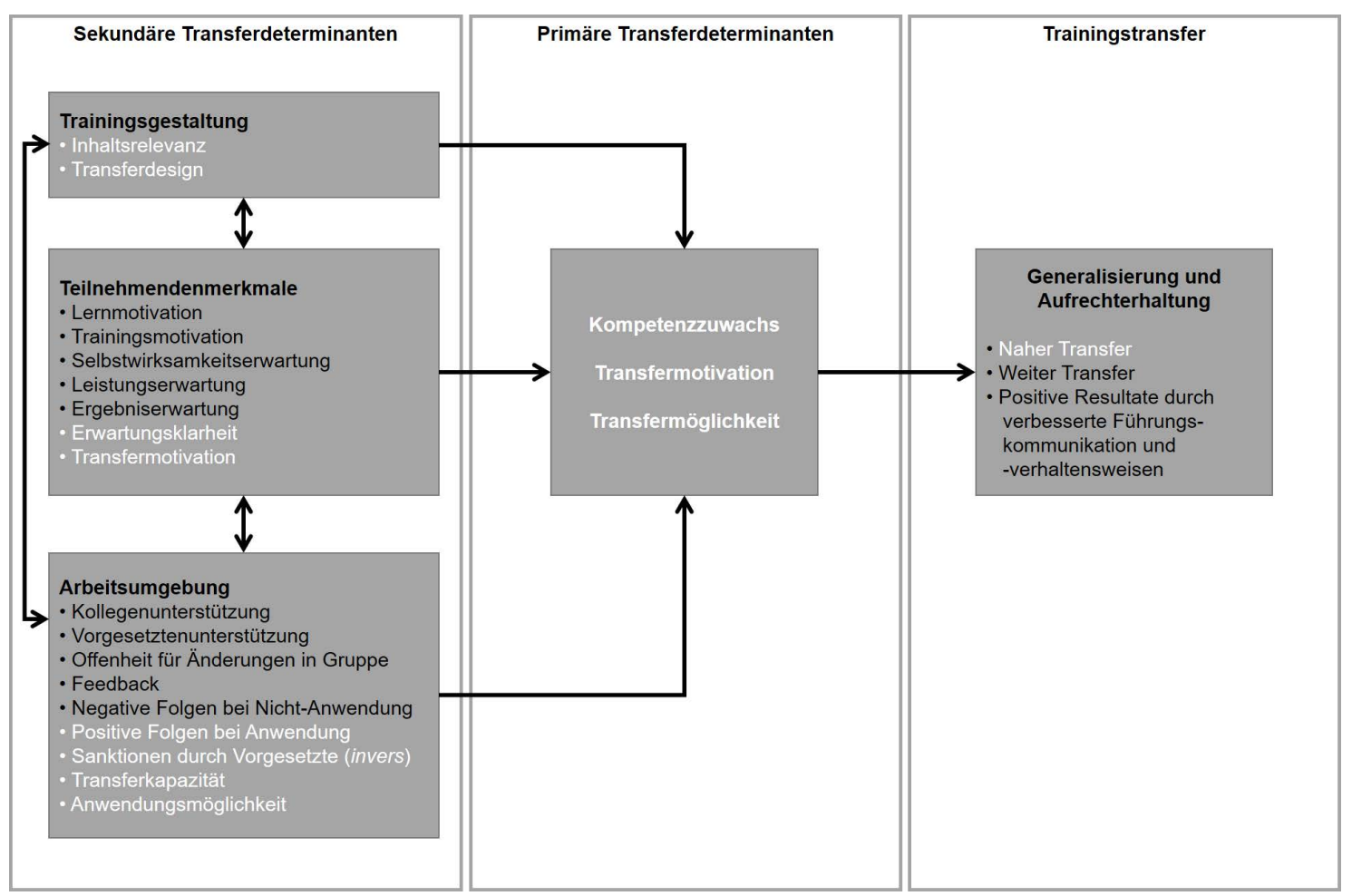

Abbildung 12: Transfermodell des Forschungsprojektes

(schwarz dargestellte Prädiktoren wirken bausteinübergreifend, weiß dargestellte Prädiktoren wirken trainingsspezifisch und werden für jeden Trainingsbaustein se-parat erfasst).

Da der Fokus des Forschungsprojektes die theoretisch fundierte Beantwortung eines sehr komplexen Wirkmechanismus umfasst, findet eine Ableitung der zu untersuchenden Hypothesen aus umfassender Theoriebildung statt. Bis heute dient das Transfermodell von Baldwin und Ford (1988) als zentrale Basis der Transferforschung. Die zahlreichen Varianten weiterer Modelle zum Trainingstransfer können weitestgehend als Modifikationen dieses Modells gelten. Dennoch ist dessen Gültigkeit bisher noch nicht umfassend empirisch überprüft. So können kaum belastbare Aussagen über den relativen Einfluss einzelner Transferfaktoren und deren Zusammenhänge getroffen werden. Auch im vorliegenden Forschungsprojekt ist es nicht möglich ein komplettes Transfermodell inferenzstatistisch zu überprüfen. Aufgrund der Komplexität des Transferprozesses mit zahlreichen Transferdeterminanten wäre eine Stichprobe mit über 300 Probanden erforderlich (Moosbrugger \& Rauch, 2009), die entsprechend der Forschungsfragen aus Führungskräften bestehen sollte bei denen transferrelevante Einflussgrößen aus Arbeitsumfeld, Trainingsgestaltung und Teilnehmendenmerkmale kontrolliert bzw. konstant gehalten werden müssen. Dass dies in Feldstudien kaum zu realisieren ist, zeigt sich auch in dem Überblick der Evaluationsforschung von Führungskräfteentwicklungsmaßnahmen (siehe Abschnitt 3.1.3 ab Seite 41). 
Trotz methodischer Vorteile großer Stichproben, wird die Priorität in diesem Forschungsprojekt auf die inhaltliche Passung der Stichprobe auf die Forschungsfragen gelegt. Da dabei der Erkenntnisgewinn über Transfermechanismen im Fokus der Erhebungen liegt, wird versucht durch Betrachtung einzelner zentraler Wirkzusammenhänge ein ganzheitliches Transfermodell wenigstens in Teilschritten an einer kleinen Stichprobe zu erforschen. Dies ermöglicht die Nutzung reichhaltiger Datensätze und eine Passung zu den Forschungsinhalten, wobei Konfundierungen (z. B. bezüglich Teilnehmender, Arbeitsumfeld, Trainier- und Evaluationsteam, Trainingsinhalte, Trainingssetting und Transferzeit) relativ umfassend kontrollierbar sind. 


\subsection{Kapitelzusammenfassung}

Gegenstand des Forschungsprojektes ist nicht nur eine maßgeschneiderte Evaluation mit längsschnittlicher Datenerhebung im Feld zur Überprüfung von Transfererfolg und -mechanismen, sondern auch die wissenschaftlich fundierte Konzeption und Durchführung von Trainingseinheiten für nachhaltig wirksame Führungskräfteentwicklung.

Die vorliegende Arbeit schlägt einen fundierten, ganzheitlichen und praxisorientierten Evaluationsansatz für die Trainingsforschung und -praxis vor, um die reichhaltigen Erkenntnisse der Trainingsforschung für die Lösung des über Jahrzehnte anhaltenden Transferproblems nutzbar zu machen. Dabei findet einerseits eine Integration von ergebnis- und prozessorientierter Überprüfung der Wirksamkeit eines evidenzbasierten Führungskräftetrainings statt. Andererseits wird der Transfererfolg auf Resultatebene nicht nur durch die Selbsteinschätzung der Teilnehmenden, sondern auch durch Fremdeinschätzungen relevanter Interaktionspartner erfasst. Die Forschungslücke prozessorientierter Transferforschung besteht insbesondere in der Überprüfung ganzheitlicher Modelle. Da basierend auf umfassenden Literaturreviews keine Studie identifiziert werden konnte, die alle drei primären Prädiktoren (Kompetenzzuwachs, Transfermotivation und -möglichkeit) erfasste, werden in Ergänzung bisheriger Forschung einerseits die bestätigten Transferdeterminanten ganzheitlich ${ }^{20}$ in das Evaluationsmodell aufgenommen, andererseits aber auch anhand einer strikten Differenzierung zwischen primären und sekundären Determinanten priorisiert.

Auf dieser Basis werden drei übergeordnete Hypothesen, die sich aus dem integrativen Transfermodell der vorliegenden Arbeit (siehe Abbildung 12 auf Seite 87) ableiten lassen, an verschiedenen Interventionen und Erfolgskriterien überprüft:

- Hypothese 1: Ein transferförderlich gestaltetes Training bewirkt (a) kurzfristigen Trainingserfolg durch positive Trainingsreaktion (Nützlichkeitseinschätzung und Transfermotivation) und Kompetenzentwicklung sowie (b) langfristigen Transfererfolg durch Transferverhalten (naher und weiter Transfer) und dessen Resultate (Verbesserung in Führungskommunikation und -verhalten).

20 Ganzheitlich im Sinne einer Erfassung aller bisher umfassend bestätigten Transferdeterminanten mit Ausnahme stabiler Persönlichkeitseigenschaften wie Gewissenhaftigkeit und emotionale Stabilität, da ihre Erhebung im Trainingskontext aufgrund der Sensibilität gegenüber Persönlichkeitstests als ungeeignet erachtet wird. 
- Hypothese 2: Transfer kann durch (a) Kompetenz, (b) Transfermotivation und (c) -möglichkeit maßgeblich vorhergesagt werden, (d) wobei Kompetenz den größten Einfluss zeigt.

- Hypothese 3: Kompetenzentwicklung, Transfermotivation und -möglichkeit können durch transferförderliche (a) Teilnehmendenmerkmale, (b) Arbeitsumgebung und (c) Trainingsgestaltung maßgeblich vorhergesagt werden, (d) wobei Trainingsgestaltung den größten Einfluss zeigt.

Zur Überprüfung dieses integrierten Evaluationsmodells wird der empfohlene Einsatz mehrerer Messzeitpunkte realisiert, um Kontrolle von Ausgangswerten und Erfassung der Wirkmechanismen über den Trainings- und Transferprozess hinweg zu ermöglichen. Zudem erscheint die Kontrolle von Trainings- und Organisationssetting für die Ableitung präziser Handlungsimplikationen wichtig, weshalb dies durch Konstanthaltung aufgegriffen wird. 


\section{$5 \quad$ Methodisches Vorgehen}

"evaluation is an art" (Cronbach, 1982)

- es gibt aus wissenschaftlicher Sicht keine perfekte Evaluation

\subsection{Einordnung des Evaluationsdesigns}

Zur übergeordneten Einordnung des Evaluationsdesigns in das Feld der Evaluationsforschung sind in diesem Abschnitt zentrale Merkmale des gewählten Evaluationsansatzes zusammengefasst. Die Wahl des Vorgehens orientierte sich dabei an einer möglichst umfassenden Umsetzung der Evaluationsstandards Nützlichkeit, Durchführbarkeit, Fairness und Genauigkeit (siehe Abschnitt 2.4 ab Seite 16). Dabei wurden folgende Ausprägungen der Evaluationsdimensionen nach Stufflebeam (2014) realisiert: Aufgrund der Vorteile insbesondere bezüglich eines direkten Einblicks in alle Phasen des Trainingsprozesses für umfassenden Erkenntnisgewinns und prozessbegleitende Optimierung ohne Informations- und Zeitverlust, wurde eine Selbstevaluation des Trainings durch die Trainerin bzw. das Trainerteam gewählt. Dies kann jedoch auch Nachteile stärkerer Verzerrungen mit sich bringen - insbesondere bezüglich selbstwertdienlicher Hypothesenformulierung und -überprüfung sowie sozialer Erwünschtheit (Scriven, 1991). Entsprechend sollte das methodische Vorgehen diesen Aspekten gezielt begegnen, um neben den Standards Nützlichkeit und Durchführbarkeit dennoch auch Genauigkeit zu realisieren. Hierfür wurde eine theoriegeleitete Programmevaluation mit den Zielen Erkenntnisgewinn, prozessbegleitende Optimierung und Erfolgskontrolle ${ }^{21}$ sowie Transferförderung unter Verwendung wissenschaftlicher Forschungsmethoden und Analyseverfahren durchgeführt. So kann das Forschungsprojekt der Disziplin bzw. Methode „Evaluationsforschung“ zugeordnet werden (Bortz \& Döring, 2009; Suchman, 1967). Neben der Theoriebasierung wurde ein relativ umfassender Ansatz durch Integration von methoden- und praxisbasierter sowie summativer und formativer Evaluation gewählt. Da jedoch keine Vergleichsintervention unter Kontrolle zentraler konfundierender Variablen evaluiert werden konnte, wurde die Trainingsreihe in einer Einzelevaluation überprüft. Schließlich wurde insbesondere zur Sicherung der Evaluationsstandards Durchführbarkeit und Fairness ein partizipativer Evaluationsansatz gewählt, indem Unternehmensvertretende bei der Planung der Evaluation sowie bei der Operationalisierung von Transferdeterminanten und Erfolgskriterien einbezogen wurden. Die Evaluationsinstrumente wurden vom Betriebsrat vorab gesichtet, Veränderungswünsche eingebracht

21 Gemäß Hauptfunktionen der Evaluation nach Stockmann (2000): Erkenntnis, Optimierung, Kontrolle, Entscheidung und Legitimation. 
und nach Anpassungen genehmigt. Somit waren insbesondere bei eingesetzten Fragebogenitems einige Modifikationen aufgrund der Betriebsratsvorgaben erforderlich. Zudem wurde nachträglich die Nutzung von Fluktuations- und Absentismusdaten vom Datenschutzbeauftragten untersagt. In Bezug auf Hypothesen und Ziele der Evaluation wurde der Autorin freie Hand gelassen. Insgesamt wurde dennoch versucht alle Evaluationserhebungen nach Nutzenvorstellungen der Beteiligten auszurichten (utilizationfocused evaluation nach Patton, 1997), um die Akzeptanz und damit Teilnahme an der Evaluation möglichst hoch zu halten. Entsprechend mussten Fragebögen möglichst kurz sein, inhaltliche Relevanz sollte ersichtlich sein, möglichst einfache Formulierung war erforderlich und Fragen sollten nicht nur der Evaluation, sondern auch den Teilnehmenden zur Förderung der Kompetenzentwicklung bzw. des Trainingstransfers dienen. Vor dem Hintergrund dieses gewählten Evaluationsansatzes ist in nachfolgendem Abschnitt der betrachtete Evaluationsgegenstand beschrieben.

\subsection{Evaluationsgegenstand}

\subsubsection{Stichprobe}

Als Stichprobe wurden Führungskräfte eines repräsentativen deutschen, international tätigen Unternehmens der Maschinenbaubranche gewählt, das ca. 450 Mitarbeitende in Verwaltung und Produktion beschäftigte. Dabei wurden alle Mitarbeitende mit Führungsverantwortung $(N=67)$ trainiert und berücksichtigt. Die Trainingsteilnahme war für alle Führungskräfte des Unternehmens verpflichtend. Die Teilnahme an der Evaluation war dagegen freiwillig. Aufgrund von Langzeitkrankheit wurde eine Führungskraft aus der finalen Stichprobe ausgeschlossen. Die dadurch realisierte Stichprobe $(N=66)$ setzte sich wie folgt zusammen: Teilnehmende waren vorwiegend männlich (89.39\%: 59 Männer, 7 Frauen) und hatten eine Berufsausbildung als höchsten Bildungsabschluss (84.85\%: 56 mit Berufsausbildung, 10 mit Hochschulabschluss). 51.52\% (34) der Teilnehmenden waren in der Produktion, 48.48\% (32) im Verwaltungsbereich tätig. Im Mittel waren die Teilnehmenden 41.80 Jahre alt $(S D=9.85)$ und hatten 8.58 Jahre Führungserfahrung $^{22}(S D=8.57)$. Da alle Führungskräfte berücksichtigt wurden, waren auch alle Hierarchieebenen des Unternehmens vertreten (von Teamleitung bis Vorstand), wobei über die Hälfte der Teilnehmenden eine Abteilungs- oder Gruppenleitung verantworteten (siehe Abbildung 13).

22 Die Führungserfahrung wurde nur von 58 der 66 Teilnehmenden angegeben. 


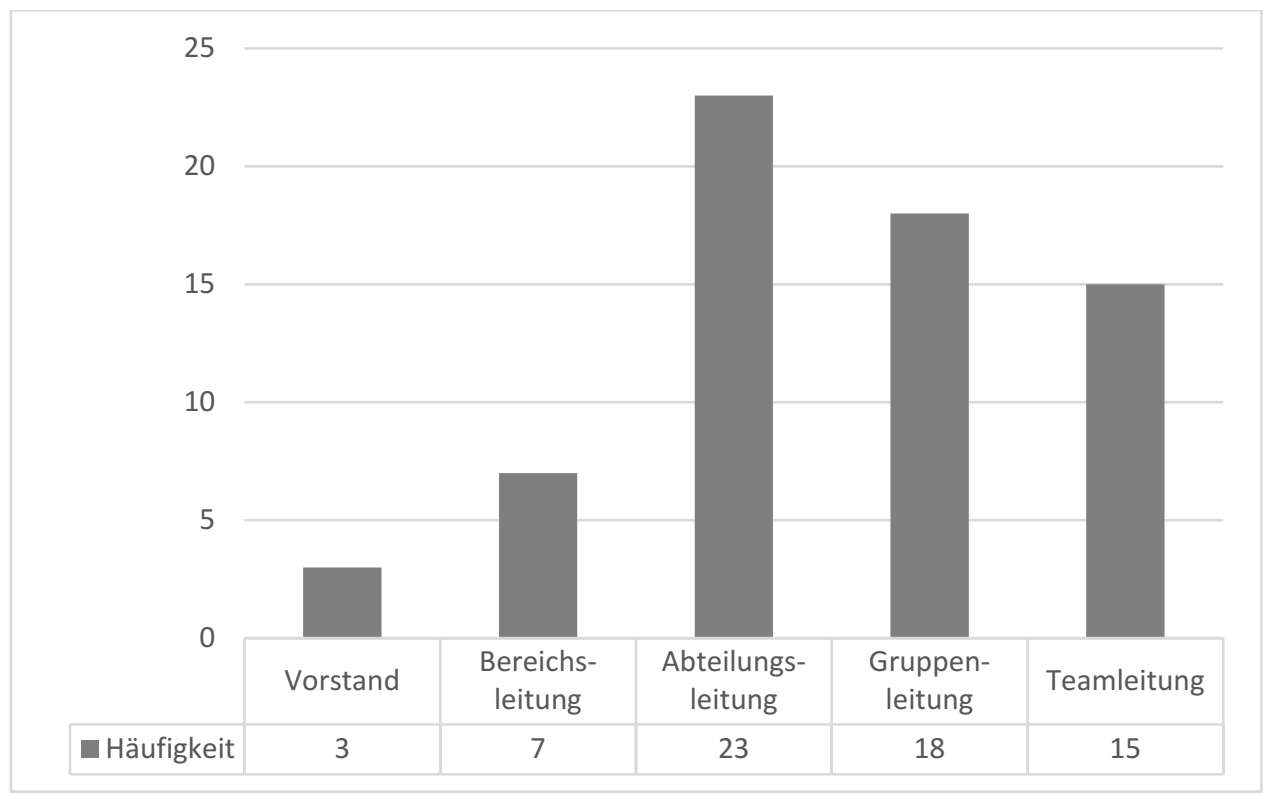

Abbildung 13: Häufigkeitsverteilung der Hierarchieebenen.

Von diesen 66 Führungskräften wurden 42 im Jahr 2014 verteilt auf vier Gruppen trainiert, wobei auf eine vergleichbare Verteilung bezüglich Kontrollvariablen und Gruppengröße zwischen 8 und 14 Teilnehmenden geachtet wurden. Die übrigen 24 Führungskräfte wurden mit der gleichen Trainingsreihe im Jahr 2016 verteilt auf zwei gleichgroßen Gruppen trainiert. Dadurch wurde ein kombiniertes Verfahren aus Eingruppen-Prä-Posttestplan und Wartelistenkontrollgruppenvergleich zur ergebnisorientierten Wirksamkeitsprüfung ermöglicht, um die jeweiligen Vor- und Nachteile zu kompensieren. Auch wenn einige Forscher ein Kontrollgruppendesign für aussagekräftige Überprüfungen eines Trainingserfolgs für erforderlich halten (Rosenstiel et al., 2005), kommen andere zu dem Schluss, dass Teilnehmendenmerkmale durch einen Pretest-Posttest-Plan verlässlicher kontrolliert werden können und daher ein Eingruppen-Prä-Posttestplan das geeignetere Design für die Überprüfung von Trainingswirksamkeit ist: Individuelle Unterschiede der Teilnehmenden, die mit der Intervention interagieren, können nur bei Within-Designs umfassend kontrolliert werden (Frese, Beimel \& Schoenborn, 2003; Hunter \& Schmidt, 1990). Zudem ist es in der organisationalen Praxis kaum möglich, genügend Probanden für eine Versuchsanordnung mit verschiedenen Interventionsbedingungen zu bekommen bzw. randomisiert auf Versuchsgruppen zu verteilen und Störvariablen ausreichend zu kontrollieren (Rosenstiel \& Nerdinger, 2011). Selbst wenn es möglich ist, eine für derartige Studiendesigns ausreichend große Stichprobe zu generieren, nimmt der Einfluss von konfundierenden Effekten im Kontext der Transferforschung von Führungskräftetrainings unkontrollierbar zu (beispielsweise durch unterschiedliche Trainierende, Arbeitskontexte, zeitliche Verschiebungen, Trainingsinhalte, o. Ä.) 


\subsubsection{Intervention}

\section{Trainingsinhalt}

Bei der fünftägigen Trainingsreihe, die im Rahmen des vorliegenden Forschungsprojektes evaluiert wurde, handelt es sich um ein relativ klassisches Führungskräftetraining bezüglich Zielsetzung und Setting. Anhand von drei Trainingsbausteinen wurden verschiedene Kompetenzen für erfolgreiche Mitarbeitergesprächsführung weiterentwickelt, wodurch übergeordnet erfolgreiche Führung (im Sinne von transformationaler, transaktionaler und interaktionaler Führung) gefördert werden sollte.

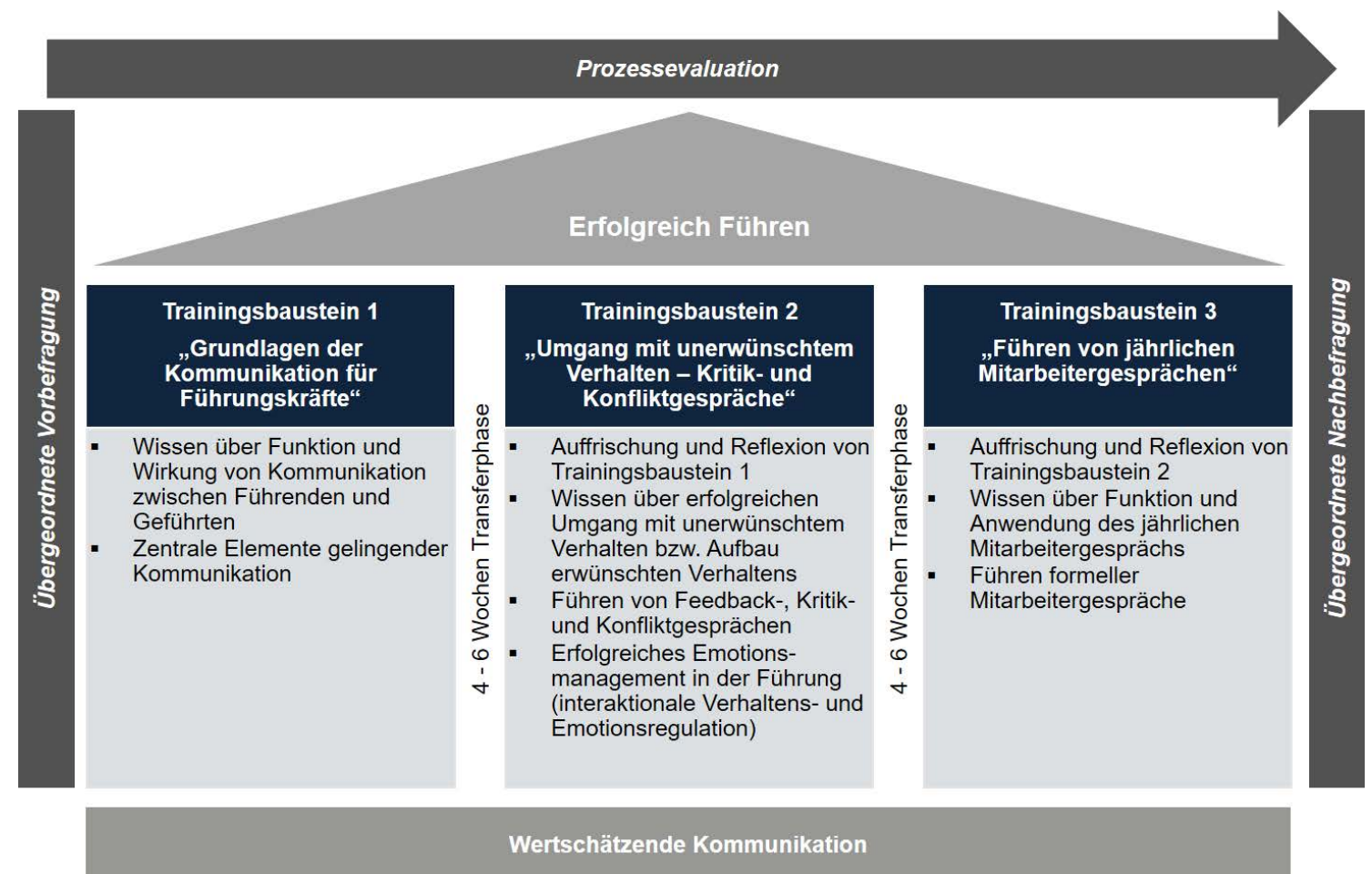

Abbildung 14: Überblick über Aufbau der Trainingsreihe zur Mitarbeitergesprächsführung; eigene Darstellung.

In Abbildung 14 sind die Inhalte der einzelnen Bausteine zusammengefasst. Der erste Baustein vermittelte im Rahmen einer eintägigen Einführungsveranstaltung Bedeutung und Grundlagen der Kommunikation für Führungskräfte (basierend auf den Elementen erfolgreicher Kommunikation nach Rogers, 1973) zur Vorbereitung (Pre-Trainingintervention) auf den zentralen Trainingsbaustein 2. Dieser sollte in einem 1,5-tägigen Training den Umgang mit unerwünschtem Mitarbeiterverhalten durch erfolgreiches Emotionsmanagement und Kompetenzen interaktionaler Verhaltens- und Emotionsregulation in der Führung vermitteln - was basierend auf Bedarfsanalysen als Hauptziel der Trainingsreihe identifiziert wurde. Da diese Kompetenzen ebenfalls Basis für die empirisch 
bestätigten erfolgreichen transformationalen, transaktionalen und interaktionalen Führungsstile sind, bestand ein übergeordnetes distaleres Trainingsziel auch in deren Förderung. Der dritte und letzte Trainingsbaustein diente mit einem Umfang von 2,5 Tagen zunächst der Wiederholung und Reflexion des zentralen Trainingsbausteins 2. Hauptbestandteil war jedoch eine Einführung des neuen jährlichen Mitarbeitergesprächs als Führungsinstrument zur Institutionalisierung der trainierten Kompetenzen. Ca. 3 Wochen vor und ca. 7 Monate nach der Trainingsreihe wurde eine Übergeordnete Vor- und Nachbefragung zur Erfassung von Transferfaktoren und -erfolg durchgeführt. Zudem fanden ca. 2 Wochen vor und unmittelbar nach den einzelnen Trainingsbausteinen weitere Erhebungen trainingsbausteinspezifischer Transferdeterminanten und kurzfristiger Erfolgskriterien für eine umfassende Prozessevaluation statt (konkrete Operationalisierungen sind in Abschnitt 5.3 ab Seite 109 beschrieben).

\section{Trainingsmethoden}

Das Vorgehen der Trainingsgestaltung orientierte sich am Prozessmodell formeller Personalentwicklung nach Solga et al. (2011), siehe Abbildung 1 in Abschnitt 2.1 ab Seite 8. Entsprechend wurde das Training mit einem systematischen Transfermanagementansatz basierend auf wissenschaftlich fundierten Erkenntnissen zur Transferförderung konzipiert. Dafür wurden die Checklisten von Salas et al. (2012) herangezogen, in denen die Erkenntnisse zur transferförderlichen Trainingsgestaltung aus der bisherigen Trainingsforschung zusammengefasst sind. Diese Handlungsempfehlungen sind zeitlich eingeteilt in Maßnahmen vor, während und nach Trainings. Diese Einteilung dient auch hier zur Strukturierung der gewählten methodischen Gestaltungsaspekte, wobei eine Zusammenfassung der Checklisten jeweils den Einstieg bilden. Wie die einzelnen Handlungsempfehlungen dieser Checkliste in diesem Forschungsprojekt umgesetzten wurden, ist daran anknüpfend beschrieben. 


\section{Transfermanagement vor Trainings}

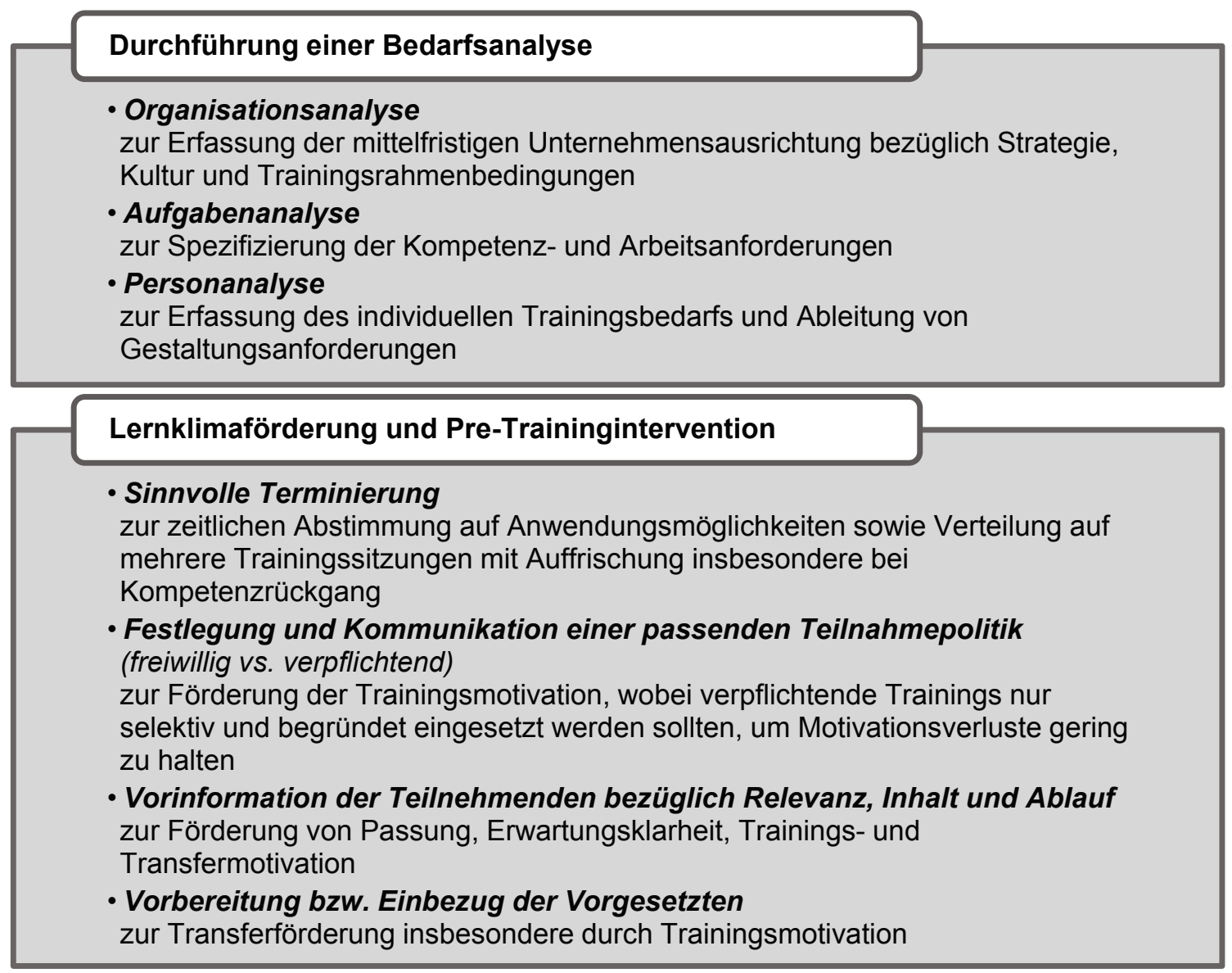

Abbildung 15: Checkliste für Maßnahmen vor Trainings in Anlehnung an Salas et al. (2012); eigene Übersetzung und Darstellung.

Wie auch im Prozessmodell formeller Personalentwicklung nach Solga et al. (2011) vorgesehen, empfiehlt die in Abbildung 15 zusammengefasste Checkliste für Maßnahmen vor Trainings von Salas et al. (2012) in einem ersten Schritt eine Bedarfsanalyse zur zielgerichteten Trainingskonzeption und -evaluation durchzuführen. Im vorliegenden Forschungsprojekt wurden zur Erfassung der mittelfristigen strategischen Ausrichtung, Normen und organisatorischen Rahmenbedingungen, Gespräche mit dem für die Trainingsreihe zuständigen Projektteam geführt. Da es aus Mitgliedern der Geschäftsführung, Betriebsrat und Personalleitung und damit aus wichtigen Entscheidungsträgern bestand, diente es als zentrale Quelle für die Organisationanalyse. Zentrales Entwicklungsziel war Unternehmenswachstum, was Teilung von Teams und mehr Führungsverantwortung im Unternehmen mit sich bringt. Dabei sollten Leitungsfunktionen zukünftig verstärkt Beziehungsgestaltung, individuelle Motivierung und Förderung sowie Stärkung einer konstruktiven Feedbackkultur in der Arbeitsgruppe als zentrale Führungsaufgaben verstehen. Dies sind Aspekte transformationaler und interaktionaler Führung. Dazu sollten auch institutionalisierte Instrumente wie das neue jährliche Mitarbeitergespräch im 
Unternehmen eingeführt werden. Da für die Geschäftsführung Personalentwicklung und insbesondere Führungskräfteentwicklung als sehr wichtig erachtete und entsprechend priorisierte, wurden ausreichend Mittel und sehr positive Rahmenbedingungen für die Trainingsreihe bereitgestellt (z. B. gut ausgestattete Räumlichkeiten, Verpflegung und Trainingszeit). Ergänzend wurde eine Analyse der Lernkultur (zusammen mit der Aufgabenanalyse) aus Sicht der Geschäftsführung, der unterstellten Mitarbeitenden und der Führungskräfte selbst in zwei Schritten erfasst: Einerseits wurden halbstrukturierte Interviews mit einer repräsentativen Stichprobe der Belegschaft aller Hierarchieebenen $(N=$ 19) verwendet. Andererseits wurden Ergebnisse der schriftlichen Mitarbeiterbefragung der gesamten Belegschaft des Unternehmens $(N=317)$ mit geschlossenen und offenen Fragen bezüglich Lernkultur und Mitarbeiterführung herangezogen. Interviewergebnisse zur Lernkultur waren grundsätzlich positiv bewertet, wobei Wünsche bezüglich 1) Förderung des gegenseitigen Verständnisses (z.B. durch Job-Rotation), 2) offenerem Umgang mit Kritik, Problemen und dazu passenden Lösungsvorschlägen sowie 3) Verbesserung der Umsetzung neuer Ideen bzw. Innovationen geäußert wurden. Die Ergebnisse der schriftlichen Mitarbeiterbefragung bezüglich Lernkultur waren eher durchmischt und mittelmäßig bewertet. Neben dem Wunsch nach Verbesserung der Umsetzung neuer Ideen und Innovationen, wurde insbesondere der Bedarf an mehr Personalentwicklungsmaßnahmen und individueller Förderung auch von Mitarbeitenden ohne Führungsverantwortung rückgemeldet.

Die Aufgabenanalyse bezüglich Anforderungen an Führungskräfte des Unternehmens sowie ihre verfügbaren Mittel zu deren Bewältigung basierten insbesondere auf den Interviews. Dabei war die Zufriedenheitseinschätzung mit der Führung sehr durchmischt. Als größte Optimierungspotentiale der Führung wurden konsequentere Nachverfolgung von Absprachen, individuelle Leistungsrückmeldung und Unterstützung bei Zielerreichung genannt, was auch Aspekte transaktionaler Führung sind. Zudem wurden Wünsche geäußert bezüglich 1) eines direktiveren und konsequenteren Durchgreifens bei unerwünschtem Mitarbeiterverhalten als Führungsaufgabe, 2) bessere Kommunikation von Entscheidungen (mehr und frühzeitige Hintergrundinformationen bzw. Begründungen), 3) klarere Priorisierung von Aufgaben durch die Führungskraft, 4) bessere Unterstützung bei Fehlerbehebung, 5) mehr Präsenz der Führung insbesondere im produzierenden Bereich, 6) weniger Ungleichbehandlung von Mitarbeitenden und 7) mehr Perspektiven durch das Aufzeigen von Zielen und Entwicklungsmöglichkeiten. Ergebnisse zur Führung aus der schriftlichen Mitarbeiterbefragung bestätigten die Interviewergebnisse und sahen ebenfalls den größten Entwicklungsbedarf der Führung in individueller Rückmeldung durch konstruktive Kritik und Anerkennung. Zudem wurde insbesondere 
das bereits aufgezeigte Problem der fehlenden Kompetenz im Umgang mit unerwünschtem Mitarbeitendenverhalten bzw. der Förderung erwünschten Verhaltens aus verschiedenen Perspektiven als handlungsrelevant benannt.

Da die evaluierte Intervention ein Basistraining für alle Führungskräfte darstellte, wurde die Personanalyse nicht zur Teilnehmendenselektion verwendet. Stattdessen diente sie der Berücksichtigung individueller Unterschiede bezüglich Fähigkeiten, Motivation und Möglichkeiten, auf die in der Trainingsdurchführung eingegangen wurde. Hierfür wurden Vorwissen, Transferfaktoren (insbesondere Trainings- und Transfermotivation) und Kontextbedingungen erfasst, um Aspekte von persönlicher Relevanz, Transfermotivation und Anwendungsmöglichkeit der Trainingsinhalte gezielt zu fördern.

Um ein lern- und transferförderliches Klima zu schaffen, wurde nach der bedarfsorientierten Zielklärung des Trainings entsprechend der Checkliste von Salas et al. (2012) eine passende Terminierung und Teilnahmepolitik festgelegt. Da die Anwendungsmöglichkeiten der festgelegten Trainingsinhalte aufgrund der in der Bedarfsanalyse aufgezeigten Relevanz und Dringlichkeit im bestehenden Arbeitsalltag der Zielgruppe gegeben sind, orientierte sich die Terminierung an der Verfügbarkeit von Teilnehmenden und Räumlichkeiten. Aufgrund der umfassenden Kompetenzen, die im Training vermittelt werden sollten, wurde ein zeitlich verteiltes Training mit Auffrischungssitzungen gewählt. Da der Führungskräfteentwicklungsbedarf über alle Hierarchieebenen und Funktionsbereiche des Unternehmens als akut identifiziert wurde, erschien eine verpflichtende Teilnahmepolitik für alle Führungskräfte zur Qualitätssicherung wichtig. Durch die Begründung basierend auf der aufgezeigten Relevanz in der Mitarbeiterbefragung sowie einer expliziten Einordnung der Trainingsreihe in die Unternehmenskulturentwicklung, sollten Motivationsverluste gegenüber freiwilliger Trainingsteilnahme kompensiert werden (siehe auch Abschnitt 3.2.1 ab Seite 65) ${ }^{23}$. Zudem wurden PreTraininginterventionen in Form von Vorinformationen bezüglich Relevanz, Inhalt und Ablauf des Trainings umgesetzt. Einerseits warben unternehmensinterne „Promotoren“ (Schlüsselpersonen aus Betriebsrat, Geschäftsführung und Personalbereichsleiter) explizit auf einer Betriebsversammlung sowie im Intranet für die Trainingsreihe, deren transferförderliche Ausrichtung und für eine aktive Teilnahme. Andererseits wurde auch Informationsmaterial vom Trainerteam einige Woche vor der Trainingsreihe an die Teilnehmenden versendet. Darin wurde über nachfolgende Aspekte informiert und ein Einverständnis der Teilnehmenden eingeholt bzw. eine Möglichkeit Änderungswüsche ein-

${ }^{23}$ Freiwilligkeit wurde als Faktor für Transfer (Kodwani \& Prashar, 2019a) bzw. für intrinsische Transfermotivation (Curado et al., 2015) identifiziert. 
zubringen gegeben. Dies sollte auch den Nachteilen der verpflichtenden Teilnahme entgegenwirken, indem dennoch die Selbstbestimmung der Teilnehmenden gewährleistet wurde. Im Folgenden sind die zentralen Inhalte, der Vorinformation für die Teilnehmenden dargestellt:

\section{Relevanz und Inhalt der Trainingsreihe}

- „Übergeordnete Ziele:

$>$ Verfeinerung Ihrer Kommunikationskompetenz,

z.B. für Feedback im Berufsalltag, bei unerwünschtem Mitarbeiterverhalten und Konflikten oder zur Mitarbeiter(motivations)förderung.

$>$ Vorbereitung auf das neue jährliche Mitarbeitergespräch, bei dem alle Aspekte flexibel und angemessen einzusetzen sind.

Unterstützung der Unternehmenskulturentwicklung.

- Die Trainingsreihe will Ihnen nicht zeigen wie Führung in Ihren jeweiligen Rahmenbedingungen „richtig“ funktioniert, da Sie dafür selbst die besten Experten sind.

- Sie soll vielmehr eine Plattform zur Selbstreflexion und Systematisierung Ihrer bereits vorhandenen Kompetenzen bieten, um diese für erfolgreiche Gesprächsführung zielgerichtet und individuell stimmig weiterzuentwickeln."

\section{Ablauf der Trainingsreihe}

- „Um die Trainings in vertrauensvoller Atmosphäre und auf Ihre individuellen Bedürfnisse zugeschnitten abhalten zu können, wurden Sie auf 6 gleichmäBig durchmischte Trainingsgruppen mit jeweils ca. 10 Teilnehmenden aufgeteilt.

- Den Gruppen werden hintereinander die gleichen Trainingsinhalte im Rahmen von jeweils 5 Trainingstagen vermittelt.

- Eine transferförderliche Gestaltung des Trainings soll Ihnen ermöglichen aus den 5 Trainingstagen möglichst nachhaltige Effekte zu ziehen, indem die Anwendung im Arbeitsalltag erleichtert wird.

- Der Erfolg hängt dabei an jedem Einzelnen von Ihnen und Ihrer aktiven Auseinandersetzung mit den Inhalten, wobei wir als Trainer die bestmögliche Unterstützung nach dem aktuellen Forschungsstand geben.

- Durch die Trainingsvorbereitung werden Sie mit Fragen zur Selbstreflexion, der Aktivierung von Vorwissen und der Erfassung Ihres aktuellen Standes der Kommunikation in das Thema eingeführt. 
- Während der Trainings fördert die Kombination aus interaktiver Vortragsform zur Wissensvermittlung und realitätsnahen Lernmöglichkeiten zur Selbsterprobung mit Feedback den Lerneffekt.

- Auch die Phasen zwischen den 3 Trainingsbausteinen sind wesentlicher Bestandteil Ihrer persönlichen Entwicklung.

- Der Abschluss der Trainingstage stellt den Beginn des Lernprozesses dar. Sie werden dabei unterstützt persönliche Entwicklungsziele zu definieren, die es dann in Ihrem Berufsalltag umzusetzen gilt.

- Ob und wie gut es Ihnen gelingt die Trainingsziele im Alltag umzusetzen, wird maßnahmenbegleitend und auch noch über das Training nach ca. 6 Monaten und einem Jahr hinaus nachverfolgt, um bei Bedarf weitere Unterstützung geben zu können.“

\section{Ablauf der Evaluation der Trainingsreihe}

- „Sie erhalten zu verschiedenen Zeitpunkten insgesamt 4 Fragebögen per Mail, die teilweise schon vor den Trainings an uns zurückzusenden sind.

- Um den individuellen Zeitaufwand dennoch möglichst gering zu halten, müssen nicht von jeder Trainingsgruppe vor jedem Baustein die gleichen Fragebögen ausgefüllt werden. Der Aufwand wurde auf die vier Gruppen gleichmäßig verteilt, sodass Sie - wo dies möglich ist - von den Antworten der anderen Teilnehmergruppen profitieren können.

- Damit das funktionieren kann, ist es wichtig, dass Sie sich immer an Ihre personalisierten Bögen und Zeitangaben halten und sich nicht an Teilnehmenden aus anderen Gruppen orientieren.

- Zur Orientierung erhalten Sie jeweils mit einem Vorlauf von ca. 10 Tagen Mails mit Ihren personalisierten Fragebögen (durch einen Code, der für jeden Teilnehmenden für alle Fragebögen vergeben wird) und Abgabeterminen.

- Bitte beantworten Sie jede Frage so ehrlich wie möglich. Wenn Sie eine Frage nicht beantworten können oder möchten, überspringen Sie diese.

- Eine Zuordnung der Antworten zu den Teilnehmenden ist ausschließlich für das Trainerteam möglich.

- Jegliche Informationen, die an Dritte gegeben werden, werden ausschließlich in anonymisierter und zusammengefasster Form übermittelt, sodass kein Rückschluss auf einzelne Angaben oder Personen möglich ist.

- Um Erkenntnisse für weitere Trainingsoptimierungen ableiten zu können, werden die anonymisierten Daten an der Universität Bamberg ausgewertet, wobei wir Ihnen einen absolut vertraulichen und gewissenhaften Umgang Ihrer Angaben garantieren." 
Schließlich diente auch die Einführungsveranstaltung (Trainingsbaustein 1) als Vorinformationsplattform: Nach einer Kurzpräsentation zur Einordnung der Trainingsreihe in die Unternehmenskulturentwicklung durch die Personal(entwicklungs)leitung, wurden die übergeordneten Vorgaben zu Relevanz, Inhalt und Ablauf zusammen mit den Teilnehmenden interaktiv feinjustiert und präzisiert. So sollte nicht nur die Akzeptanz der Trainingsreihe, sondern auch ihre Bedarfsorientierung top-down und bottom-up sichergestellt werden.

Für Vorbereitung bzw. Einbezug der Vorgesetzten der Teilnehmenden wird beispielsweise eine Beteiligung der Führungskräfte an der Bedarfsanalyse, eine Aufforderung und Anleitung zur Durchführung von Entwicklungsgesprächen oder das Aufzeigen der Trainingsrelevanz oder Optimierung von Transferfaktoren der Arbeitsumgebung durch die Führungskräfte empfohlen (siehe auch Kauffeld, Grote \& Frieling, 2009; Kauffeld et al., 2012). Dadurch soll insbesondere die Trainings- und Transfermotivation zusätzlich gefördert werden. Im Rahmen des vorliegenden Forschungsprojektes war dies durch die sonstigen Maßnahmen bereits abgedeckt, da alle Führungskräfte auch Teilnehmende waren und daher direkt in die Förderung von Trainings- und Transfermotivation eingebunden waren. 


\section{Transfermanagement während Trainings}

Förderung Teilnehmendeneinstellung

- Motivierung durch Aufzeigen persönlicher Relevanz bzw. des individuellen Nutzens des Trainings

zur Förderung der Lerneinstellung, Ermutigung zum Transfer

- Förderung der Lernorientierung durch Verstärkung aktiver Teilnahme und Eigenverantwortlichkeit

zur Förderung der Kompetenzentwicklung

- Verstärkung von Selbstwirksamkeit durch Ermutigung und Ermöglichung von Leistungserfolg

zur Förderung von Transfermotivation und -durchhaltevermögen

Passgenauer Einsatz methodischer Prinzipien

- Effektive(s) und fundierte(s) Design und Trainingsstrategie durch

Kombination aus Wissensvermittlung, positiven und negativen

Verhaltensbeispielen, Übungsmöglichkeiten und Feedback

zur Förderung des Verstehens und Anwendens der Trainingsinhalte

- Förderung von Selbstregulation durch Ermutigung zur Selbstreflexion

und -kontrolle sowie Aufmerksamkeitsausrichtung auf Aufgaben

zur Unterstützung der Selbstkontrollfähigkeiten bei Zielerreichungsprozessen

- Raum für Fehler in geschütztem Rahmen, um daraus gemeinsam zu lernen

zur Transferförderung durch Kompetenzaufbau im Umgang mit Hindernissen und Herausforderungen

- Individuelle und strukturierte Lernerfolgskontrollen

zur Verstärkung der Lernerfahrung und Verdeutlichung des Lernerfolgs

- Lernförderung durch Simulation komplexer oder kritischer Situationen

zur Förderung des Verständnisses von Zusammenhängen und

Handlungskompetenz

- Transfervorbereitung durch Abstimmung von Lern- und Arbeitsfeld (inhaltlich, bezüglich Variabilität und Schwierigkeit)

zur Erleichterung der Umsetzung des Gelernten im Arbeitsalltag

- Sinnvoll unterstützende Verwendung von Technologie zur

Wissensvermittlung

zur Optimierung und Ermöglichung individuellen, selbstbestimmten Lernens

Abbildung 16: Checkliste für Maßnahmen während Trainings in Anlehnung an Salas et al. (2012); eigene Übersetzung und Darstellung.

Die in Abbildung 16 zusammengefasste Checkliste für Maßnahmen während Trainings nach Salas et al. (2012) wurde ebenfalls möglichst umfassend und praxistauglich umgesetzt. Die Unterstützung transferförderlicher Teilnehmendeneinstellung wurde über den gesamten Trainingsprozess forciert. Anknüpfend an die beschriebene Vorbereitung des Lernklimas bereits vor dem Training, wurden die Teilnehmenden zu Beginn der Trainingsreihe darin angeleitet in 2-3er-Gruppen ihr persönliches Entwicklungsziel für die Trainingsreihe zu definieren (siehe Anleitung zur gemeinsamen Reflexion in Abbildung 17). 


\section{Übergeordnetes Entwicklungsziel}

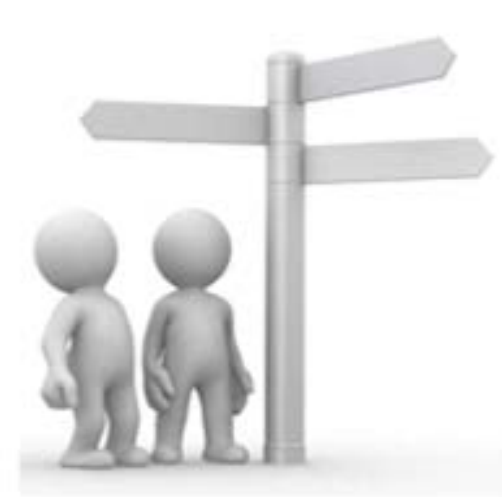

Leitfragen:

Was ist mein individuelles Entwicklungsziel?

Was genau zum übergeordneten Thema Mitarbeitergesprächsführung möchte ich klären/verăndern?

Aufgabe Partnerinterview:

Bitte interviewen Sie sich gegenseitig zu Ihrer aktuellen Führungssituation und unterstützten Sie| sich gegenseitig darin, ein Thema/Ziel als individuellen Fokus für die Trainingsreihe auszuwåhlen.

Abbildung 17: Anleitung zur gemeinsamen Reflexion persönlicher Entwicklungsziele.

Dieses persönliche Entwicklungsziel diente während der gesamten Trainingsreihe als Referenzpunkt, indem wiederholt reflektiert wurde inwiefern die Trainingsinhalte genutzt und angewendet werden können, um diesem Ziel näher zu kommen bzw. weitere Zielaspekte zu definieren. Dadurch sollte einerseits die Motivierung zur aktiven Teilnahme sowie zum Transfer durch kontinuierliches Aufzeigen der persönlichen Relevanz bzw. des individuellen Nutzens des Trainings gefördert werden.

Förderung der Lernorientierung mittels Verstärkung aktiver Teilnahme und Eigenverantwortlichkeit wurde nach der Pre-Trainingintervention beispielsweise durch vorwiegenden Einsatz aktiver Trainingsmethoden (Diskussionen, Murmel- bzw. Kleingruppenarbeit und Behavior Modeling, siehe auch Abschnitt 2.1 ab Seite 7), Anwendungsorientierung (z. B. durch Beispiele und Rollenspiele zu Umsetzung, Hindernissen und deren Lösungsmöglichkeiten), individueller Rückmeldung der Ergebnisse einer Kurzform des Multifactor Leadership Questionnaire (MLQ 5x von Bass und Avolio (1995) bzw. dessen deutsche Übersetzung nach Felfe und Goihl (2002) sowie Felfe (2006)) basierend auf Selbstund Mitarbeitendeneinschätzung sowie durch die explizite Übertragung von Transferverantwortung an die Teilnehmenden umgesetzt. Zudem wurde das generelle Trainingsverständnis im Sinne eigenverantwortlicher Lernorientierung als Plattform zur Selbstreflexion, Systematisierung und Erweiterung bereits vorhandener Kompetenzen im Rahmen der Vorinformation durch folgende Hinweise vorbereitet:

„Damit für diese 5 von Ihnen zu investierenden Trainingstage ein möglichst nachhaltiger Trainingseffekt realisiert werden kann, ist das gesamte Trainingskonzept auf eine transferförderliche Gestaltung ausgerichtet, sodass eine Anwendung im Arbeitsalltag für Sie erleichtert wird. Neben der Nutzenmaximierung der Trainingsreihe wurde dabei auf eine möglichst handhabbare mit geringem Aufwand für Sie verbundene Umsetzung geachtet. 
Der Erfolg hängt dabei an jedem Einzelnen von Ihnen und Ihrer aktiven Auseinandersetzung mit den Inhalten, wobei wir als Trainer die bestmögliche Unterstützung nach dem aktuellen Forschungsstand geben.

Dazu gehört unter anderem, dass das Training nicht mit den festgelegten Trainingstagen beginnt und endet, sondern bereits zur Trainingsvorbereitung mit Fragen zur Selbstreflexion, Aktivierung von Vorwissen und Erfassung Ihres aktuellen Standes der Kommunikation eingeleitet wird. Auch der Abschluss der Trainingstage stellt nicht das Ende, sondern den Beginn des Lernprozesses dar, bei dem sie darin unterstützt werden persönliche Entwicklungsziele zu definieren, die es dann in Ihrem Berufsalltag umzusetzen gilt. So sind auch die Phasen zwischen den drei Trainingsbausteinen wesentlicher Bestandteil Ihrer persönlichen Entwicklung. Ob und wie gut dies Ihnen gelingt die Trainingsziele im Alltag umzusetzen, wird maßnahmenbegleitend und auch noch über das Training hinaus nachverfolgt (nach einem halben Jahr und einem Jahr), um bei Bedarf weitere Unterstützung geben zu können.“

Verstärkung von Selbstwirksamkeit durch Ermutigung und Ermöglichung von Leistungserfolg wurde insbesondere umgesetzt durch kontinuierliches prozessbegleitendes Aufzeigen bisheriger Entwicklungsschritte (z. B. durch Reflexionsrunden und Zusammenfassung der neuen Erkenntnisse und deren Relevanz für individuelle Entwicklungsziele nach jedem übergeordneten Inhaltsblock), Lernerfolgskontrollen (z. B. durch Quizzrunden, Teilnehmendenpräsentationen zur Wiederholung von Inhalten, Evaluationsbögen unmittelbar nach den Trainingsbausteinen), Anwendungs- und Übungsgelegenheiten während und zwischen den Trainingsbausteinen mit ressourcenorientiertem Feedback (durch das Trainerteam und andere Teilnehmenden im Rahmen angeleiteter Feedbackrunden zur Sicherstellung konstruktiven Feedbacks) sowie individuelle Zielsetzungsunterstützung und Förderung von Selbstmanagementstrategien bezüglich Trainingstransfer.

Zur Umsetzung eines passgenauen Einsatzes methodischer Prinzipien zu Trainingssetting und -inhalt sowie zu individuellen Teilnehmendeneigenschaften, wurden einerseits als erfolgreich identifizierte Trainingsmethoden bzw. fundiertes Trainingsdesign durch Kombination aus Wissensvermittlung, positive und negative Verhaltensbeispiele, Übungsmöglichkeiten und Feedback eingesetzt (siehe auch Abschnitt 3.2.1 ab Seite 65). Übergeordnet orientierte sich die Trainingsstrategie an transferrelevanten Lerntheorien insbesondere an der Theorie identischer Elemente nach Thorndike und Woodworth (1901), der Übertragung von Prinzipien nach Judd (1908) sowie an situiertem Lernen nach Lave und Wenger (1991) bzw. Mandl, Gruber und Renkl (2002). Daher wurden Ansätze zur Förderung selbstgesteuerten Lernens (Cappetta \& Paolino, 2015; Lourenco \& Ferreira, 2019; Sua Wui et al., 2018), Behavior Modeling (mit positiven und negativen 
Verhaltensmodellen), Error-Management- und Teambased-Ansätze (Hart et al., 2019; Taylor et al., 2005) im Rahmen von zeitlich verteilter Vermittlung mit zunehmendem Schwierigkeitsgrad umgesetzt. Andererseits wurde auf individuelle Unterschiede bezüglich Persönlichkeit und Fähigkeiten, Lernorientierung, Motivation und Selbstwirksamkeit durch adaptives Training eingegangen (z. B. durch freie Wahl von Aufgabenalternativen und Schwierigkeitsgraden, Bezug zur persönlichen Relevanz und individuellen Arbeitskontexten, individuelles Feedback, Lerngruppen in denen Erfahrenere Teilnehmenden weniger Erfahrene in ihrer Entwicklung unterstützten, Arbeit in Kleingruppen, die von CoTrainern angeleitet wurden um Individuumsorientierung zu ermöglichen). Dadurch wurden die weiteren Aspekte der Checkliste nach Salas et al. (2012) wie Förderung von Selbstregulation, Raum für Fehler in geschütztem Rahmen, Lernerfolgskontrollen und Simulation komplexer Situationen sichergestellt. Andererseits wurde inhaltlich ein großer Fokus auf die Abstimmung von Lern- und Arbeitsfeld gelegt. Dafür wurden für alle Trainingsinhalte Praxisbeispiele aus Bedarfsanalyse und Reflexionsbögen vorab erfragt und um Rückmeldungen der Teilnehmenden, die im Training eingebracht wurden, ergänzt (z. B. Inhalte der Rollenspiele für realistische Gesprächssituationen, Erfahrungsberichte in Diskussionsrunden, Anwendungsbeispiele in Zwischenreflexionsrunden zu Transfermöglichkeiten und -hindernisse). Unterstützende Verwendung von Technologie wurde durch ergänzende Online-Learning-Methoden zur Auffrischung, Erinnerung und Reflexionsanleitung der Trainingsinhalte und ihrer Anwendung auch zwischen den Trainingsbausteinen umgesetzt (z. B. durch Erinnerungsmails, Fragebögen und Onlinedokumente). 


\section{Transfermanagement nach Trainings}

Transferförderung und Post-Traininginterventionen

- Beseitigung von Transferhindernissen bzw. Aufzeigen von

Anwendungsmöglichkeiten

zur Förderung von Transfermotivation und realisierten

Anwendungsmöglichkeiten sowie Verringerung des Kompetenzrückgangs

- Bereitstellung von Instrumenten, Hilfsmittel und Handlungsempfehlungen zur Transferunterstützung durch Vorgesetzte und Teilnehmende zur Förderung der Kompetenzanwendung und Leistung im Arbeitsalltag

- Anregung zu Reflexion und Erfahrungsaustausch bezüglich Umsetzung von Trainingsinhalten im Arbeitsalltag zur Förderung von weiterem Kompetenzaufbau, Transfermotivation und -durchhaltevermögen im Arbeitsalltag

Nachhaltige Trainings- und Transferevaluation

- Klare Spezifizierung des Evaluationsziels und konsequente Ausrichtung darauf zur Sicherstellung von Effizienz und Aussagekraft der Evaluation

- Erfassung multidimensionaler Evaluationskriterien (Reaktion, Lernen, Transferverhalten und -resultate) unter Berücksichtigung präziser kognitiver, affektiver und verhaltensorientierter Indikatoren basierend auf der Bedarfsanalyse

zur Förderung einer fundierten Entscheidungsbasis für Optimierungen und Trainingsfortführung

Abbildung 18: Checkliste für Maßnahmen nach Trainings in Anlehnung an Salas et al. (2012); eigene Übersetzung und Darstellung.

Die Umsetzung der Checkliste für Maßnahmen nach Trainings gemäß Salas et al. (2012) wurden bereits vor und während des Trainings vorbereitet. Einerseits wurde der Trainingstransfer bereits in den Pre-Traininginterventionen sowie auch im Trainingsverlauf als Hauptziel explizit fokussiert und die Transferverantwortung des Unternehmens (Verantwortung für Sicherstellung einer transferförderlichen Arbeitsumgebung durch Möglichkeiten, Unterstützung und Konsequenzen) sowie der einzelnen Teilnehmenden (Verantwortung für die Wahrnehmung von Anwendungsgelegenheiten der Trainingsinhalte im Sinne des persönlichen Entwicklungsziels sowie für den erfolgreichen Umgang mit Hindernissen und Lösungsmöglichkeiten) nach jedem Inhaltsblock verdeutlicht. Andererseits wurden nicht nur die Kompetenzentwicklung der Trainingsinhalte, sondern auch wissenschaftlich fundierte Transferfaktoren prozessbegleitend erfasst, mit den Teilnehmenden reflektiert und optimiert.

Auf dieser Basis wurde die Förderung von Transferverhalten durch Post-Traininginterventionen unmittelbar nach den jeweiligen Trainingsbausteinen unterstützt. Übergeordnet orientierte sich das Vorgehen an der Zielsetzungstheorie (Locke \& Latham, 1990), der Theorie intentionaler Handlungssteuerung (Gollwitzer, 1993, 1999) bzw. Ansätzen 
zur Rückfallprävention bezüglich gewohnter Verhaltensweisen aus der Suchtintervention. Einen Überblick über den Forschungsstand zu Post-Traininginterventionen geben Rahyuda et al. (2018).

Dafür wurden die Teilnehmenden mittels Karteikarten (siehe Abbildung 19) angeleitet, ein bis drei SMARTe Entwicklungsschritte zur persönlichen Nachverfolgung zu definieren (siehe auch Kolb, 2010), um dem übergeordnete Entwicklungsziel näher zu kommen. Dadurch wurden die Teilnehmenden in proximaler und distaler Zielsetzung unterstützt, was für Aufrechterhaltung und Selbstwirksamkeit bezüglich Zielerreichungsanstrengungen wichtig ist (Brown, McCracken \& Hillier, 2013; Brown \& Warren, 2014; Brown, Warren \& Khattar, 2016).

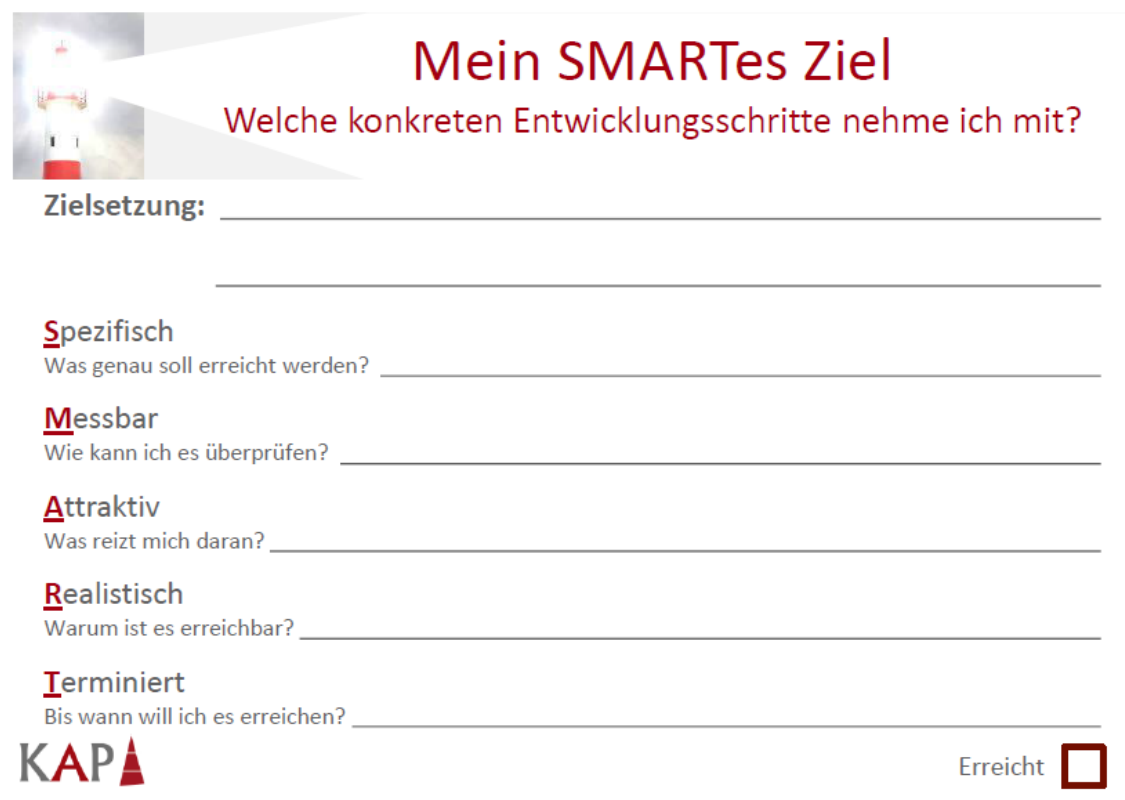

Abbildung 19: Karte zur Definition SMARTer Entwicklungsschritte mit Nachverfolgung.

Auf Basis dieser Karten wurden Möglichkeiten zur Umsetzung und Beseitigung von Transferhindernissen sowie Anwendungsmöglichkeiten reflektiert. Gleichzeitig sollten die persönlichen Karten als zentrale Instrumente, Hilfsmittel und Handlungsempfehlungen zur Transferförderung dienen. Daher wurden sogenannte Austauschpartner definiert, mit denen die Teilnehmenden nach dem Training ihre Zielerreichung anhand der Karten überprüfen und gegebenenfalls anpassen sollten. Durch Erinnerungsmails wurden Nachverfolgung und Austausch zusätzlich unterstützt. Dadurch wurde auch der Aspekt Anregung zu Reflexion und Erfahrungsaustausch bezüglich Umsetzung von Trainingsinhalten im Arbeitsalltag umgesetzt. Eine weitere Post-Trainingintervention für die ersten beiden Bausteine bestand in einer Auffrischungssitzung jeweils zu Beginn des nachfolgenden Trainingsbausteins. Dabei wurde nach einer aktiven Wiederholung durch Präsentationen der Teilnehmenden der Umsetzung- und Zielerreichungsgrad basierend auf den persönlichen Entwicklungszielen und SMART-Karten reflektiert 
und Erfahrungsaustausch zum Transfererfolg und dessen Hindernissen angeleitet. Für die gesamte Trainingsreihe, inklusive dritten Baustein, gab es zusätzlich eine freiwillige Post-Trainingintervention durch individuelle Rückmeldegespräche in denen Reflexion von Transfererfolg und Rahmenbedingungen in einem Beratungsformat mit der Trainerin bearbeitet wurden. Für eine nachhaltige Trainings- und Transferevaluation wurde mit der Spezifizierung und Ausrichtung auf das Evaluationsziel basierend auf den inhaltlichen Trainingszielen aus der Bedarfsanalyse sowie dem übergeordneten Transferfokus ein integrativer Evaluationsansatz mit formativen (prozessorientierten) und summativen (ergebnisorientierten) Kriterien gewählt. Somit wurden als multidimensionale Evaluationskriterien wie von Salas et al. (2012) empfohlen die vier Ebenen der Trainingswirksamkeit nach Kirkpatrick $(1967,1998)$ mit kognitiven, affektiven und verhaltensorientierten Indikatoren prozessbegleitend betrachtet. Zudem wurde die Dimension „Transferverhalten“ weiter spezifiziert und, entsprechend der empfohlenen Differenzierung in „nahen“ und „weiten“ Transfer, ebenfalls multidimensional und nach angemessener Transferzeit (mehr als 6 Monate nach dem Training) erfasst (Laker, 1990). Darüber hinaus wurden auch fundierte Transferdeterminanten multidimensional erfasst (siehe Operationalisierung in Abschnitt 5.3 ab Seite 109). Dieser Ansatz zielte nicht nur auf eine Wirksamkeitsüberprüfung, sondern auch auf eine Steigerung von Lern- und Transfererfolg durch wiederholte Reflexion und Auffrischung der Evaluationskriterien ab (Kodwani \& Prashar, 2019a; Li \& Wiriyakitjar, 2018; Saks \& Burke, 2012).

Durch diese Umsetzung der Checklisten für Maßnahmen vor, während und nach Trainings nach Salas et al. (2012) wurden auch die übergeordneten Gestaltungsempfehlungen aus der Trainingsforschung berücksichtigt, die beispielweise auch von Tonhäuser und Bücker (2016) oder Ford et al. (2018) zusammengefasst wurden. Auch die Empfehlungen spezifisch für nachhaltige Führungskräfteentwicklung nach Lacerenza et al. (2017) wurden in diesem Trainingskonzept realisiert (siehe Abschnitt 3.2.1 ab Seite 65):

- Einsatz formeller Führungskräfteentwicklungsmaßnahmen durch Trainings statt informelle Führungskräfteentwicklung

- Nutzung von Bedarfsanalysen als Basis für Trainingsziele

- Einsatz von Feedback und didaktischem Methodenmix (insbesondere mit Übungsmöglichkeiten) in der Trainingsdurchführung

- Zeitliche Verteilung der Trainingsmaßnahmen auf mehrere Sitzungen

- Durchführung von Präsenztrainings (statt rein virtuelle Trainingsformen)

- Qualitätssicherung durch prozessbegleitende Evaluation der Trainingsgestaltung in Bezug auf die gewünschten Ziele

Wie anhand des hier beschriebenen Evaluationsgegenstandes die Forschungsfragen möglichst umfassend überprüft wurden, ist in nachfolgendem Abschnitt beschrieben. 


\subsection{Operationalisierung}

\subsubsection{Datenquelle}

Die Forschungsfragen wurden basierend auf einer längsschnittlichen Fragebogenerhebung zur Erfassung zentraler Transferfaktoren sowie intraindividueller Veränderungsmessung bezüglich Trainings- und Transfererfolg überprüft. Dafür wurden Selbsteinschätzungen der Teilnehmenden als zentrale Datenquelle gewählt. Denn für den Trainings- und Transfererfolg sind vorwiegend „innere Zustände“ der Teilnehmenden entscheidend, die nur durch die Teilnehmenden selbst bewertet werden können (Chiaburu, Dam \& Hutchins, 2010; Facteau, Dobbins, Russell, Ladd \& Kudisch, 1995; Podsakoff et al., 2012; Spector, 2006). So ist auch bei der Trainingsgestaltung und Arbeitsumgebung nicht entscheidend, ob sie "objektiv" transferförderlich sind, sondern dass sie von den Teilnehmenden tatsächlich als transferförderlich wahrgenommen werden. Für die Transferresultate ist jedoch gerade bei Trainings von Führungsverhalten wichtig, dass die Trainingseffekte auch von den davon betroffenen Mitarbeitenden als positiv erlebt werden. Daher wurden auf der Resultatebene ergänzende Fremdeinschätzungen von unterstellten Mitarbeitenden durch direkte Veränderungsmessungen erfasst ${ }^{24}$. Durch diese sollen die Ergebnisse zum Transfererfolg basierend auf Selbsteinschätzungen „objektiviert“ beziehungsweise validiert werden, da Selbsteinschätzungen den Transfererfolg tendenziell höher bewerten als Fremdeinschätzungen (Taylor et al., 2009). Um den Common-Method-Bias der Selbsteinschätzungsvariablen möglichst gering zu halten, wurden die gängigen Empfehlungen dazu in der Fragebogenkonstruktion berücksichtigt (Podsakoff, MacKenzie, Lee \& Podsakoff, 2003; Podsakoff et al., 2012): Z. B. Durchmischung positiver und negativer (invertierter) Items oder (psychologische) Trennung von Prädiktoren und Kriterien durch explizite Trennung des Fragebogens in Reflexion von Transferdeterminanten versus Reflexion von Führungskompetenzen und -verhalten sowie Nutzung unterschiedlicher Messzeitpunkte im Trainings- und Transferprozess:

- T0

- TB1_T1

- TB1_T2

- TB2_T1

- TB2_T2

- TB3_T1

- TB3_T2

- $\mathrm{T} 3$ ca. 3 Wochen vor der Trainingsreihe (bzw. ca. 8 Wochen vor TB2)

ca. 2 Wochen vor Trainingsbaustein 1

unmittelbar nach Trainingsbaustein 1

ca. 2 Wochen vor Trainingsbaustein 2

unmittelbar nach Trainingsbaustein 2

ca. 2 Wochen vor Trainingsbaustein 3

unmittelbar nach Trainingsbaustein 3

ca. 7 Monate nach der Trainingsreihe (bzw. ca. 12 Monate nach T0)

2411 Teilnehmende hatten eine Mitarbeitendeneinschätzung, 22 Teilnehmende hatten zwei Mitarbeitendeneinschätzungen, je 1 Teilnehmende hatte drei bzw. vier Mitarbeitendeneinschätzungen. Bei mehreren Mitarbeitendeneinschätzungen zum Transfererfolg einer Führungskraft wurde der Mittelwert der Rückmeldungen verwendet. 


\subsubsection{Rücklaufquote}

Die Rücklaufquoten der Fragebögen von den 66 Teilnehmenden sind in Tabelle 5 zusammengefasst und lagen zu den jeweiligen Messzeitpunkten zwischen 59.1\% und 98.5\% (Mittelwert $=76.7 \%$ ). Da nicht alle Teilnehmenden zu allen Messzeitpunkten befragt werden konnten (siehe Status „Nicht erfragt“) ${ }^{25}$, liegen die tatsächlich ausgebliebenen Rückläufe zwischen $0 \%$ und 37.9\% (Mittelwert $=13.6 \%$ ). In Studien zur prozessorientierten Transferforschung der letzten fünf Jahre ${ }^{26}$, die Angaben zur Rücklaufquote enthalten, lag die Rücklaufquote zwischen $13 \%$ und $98 \%$ und durchschnittlich bei etwa $60 \%$. Entsprechend können die Rücklaufquoten aller Messzeitpunkte als gut bis sehr gut betrachtet werden. Daher sind rücklaufbedingte Verzerrungen aufgrund von so genannten Abdeckungs- und Nonresponsefehlern in der vorliegenden Studie als relativ gering einzuschätzen (siehe auch Modell des Total Survey Error; Groves, Dillman, Eltinge \& Little, 2002; Groves, Fowler Jr., Couper \& Lepkowski, 2009).

Tabelle 5: $\quad$ Überblick der Rücklaufquoten

\begin{tabular}{|c|c|c|c|c|c|c|c|c|c|c|c|c|c|c|c|c|}
\hline \multirow{2}{*}{ Status } & \multicolumn{2}{|c|}{ TO } & \multicolumn{2}{|c|}{ TB1_T1 } & \multicolumn{2}{|c|}{ TB1_T2 } & \multicolumn{2}{|c|}{ TB2_T1 } & \multicolumn{2}{|c|}{ TB2_T2 } & \multicolumn{2}{|c|}{ TB3_ T1 } & \multicolumn{2}{|c|}{ TB3_T2 } & \multicolumn{2}{|c|}{ T3 } \\
\hline & N & $\%$ & N & $\%$ & N & $\%$ & N & $\%$ & N & $\%$ & N & $\%$ & $\mathrm{~N}$ & $\%$ & $\mathrm{~N}$ & $\%$ \\
\hline Rücklauf & 65 & 98.5 & 39 & 59.1 & 60 & 90.9 & 51 & 77.3 & 55 & 83.3 & 50 & 75.8 & 45 & 68.2 & 40 & 60.6 \\
\hline Kein Rücklauf & 0 & 0.0 & 6 & 9.1 & 4 & 6.1 & 5 & 7.6 & 7 & 10.6 & 6 & 9.1 & 14 & 21.2 & 25 & 37.9 \\
\hline Nicht erfragt & 1 & 1.5 & 21 & 31.8 & 2 & 3.0 & 10 & 15.2 & 4 & 6.1 & 10 & 15.2 & 7 & 10.6 & 1 & 1.5 \\
\hline
\end{tabular}

Anmerkungen. $\mathrm{N}=$ Anzahl von Teilnehmenden, die den Fragebogen zum Messzeitpunkt (nicht) beantwortet haben. T0 = ca. 3-5 Wochen vor Beginn der Trainingsreihe; T3 = ca. 7 Monate nach der Trainingsreihe bzw. ca. 12 Monate nach T0; TBx_T1 = ca. 2 Wochen vor dem Trainingsbaustein $\mathrm{x} ; \mathrm{TBx} \_\mathrm{T} 2=$ unmittelbar nach dem Trainingsbaustein $\mathrm{x} ; \mathrm{TBx} \mathrm{T}_{-} \mathrm{T} 1=\mathrm{T} 0$ und TBx_T1.

\subsubsection{Umgang mit fehlenden Werten}

Wie bei Erhebungen mit Mehrfachmessungen zu erwarten - insbesondere, wenn sie über einen längeren Zeitraum verteilt stattfinden - weisen auch die Datensätze der vorliegenden Forschungsarbeit fehlende Werte durch nicht erfolgten Rücklauf ganzer Fragebögen (siehe Abschnitt 5.3.2 ab Seite 110) oder auch Nichtbeantwortung einzelner Fragen auf. Als „State-of-the-Art“ für den Umgang mit fehlenden Werten gelten modellbasierte Verfahren und insbesondere imputationsbasierte Verfahren (Single oder Multiple Imputation), um dennoch mit kompletten Datensätzen rechnen zu können (Enders,

25 Bei T0 waren die vom Unternehmen übermittelten Kontaktdaten eines Teilnehmers falsch, weshalb er den Fragebogen nicht erhielt. Bei T1 von TB1, TB2 und TB3 wurden jeweils 1-2 Trainingsgruppen nicht befragt, um den Befragungsumfang für die Teilnehmenden im vom Unternehmen vorgegebenen Rahmen zu belassen. Bei TB3 wurde ein Teilnehmer nicht befragt, da er das Unternehmen verlassen hatte.

26 Als Studienbasis wurde dieselbe Auswahl wie unter Abschnitt 3.2 ab Seite 43 beschrieben gewählt. 
2010). Da die vorliegende Stichprobe für modellbasierte Verfahren zu klein war, wäre im vorliegenden Forschungsprojekt die Multiple Imputation das passendste Verfahren ${ }^{27}$. Es ist ein Verfahren, dass bereits 1976 von Rubin vorgeschlagen wurde und auch noch heute als eines der fortschrittlichsten statistischen Verfahren zur Behandlung fehlender Werte insbesondere für den Einsatz von Regressionsanalysen gilt (Urban \& Mayerl, 2018). Allerdings ist die Voraussetzung für den sinnvollen Einsatz von Multipler Imputation, dass die fehlenden Werte ein gewisses Zufallsmuster aufweisen. Bereits aus der Rücklaufübersicht (siehe Tabelle 5 auf Seite 110) geht allerdings hervor, dass gerade bei der Erfassung des Transfererfolgs zu T3 ein großer Anteil der Datensätze durch geschätzte Werte ergänzt werden müsste. Dabei ist anzunehmen, dass es einen Zusammenhang zwischen Rücklauf und Prädiktor- sowie Kriteriumsvariablen gibt (insbesondere mit Transfermotivation, -kapazität oder -erfolg) und damit diesbezüglich ein sogenanntes „Missing Not at Random“-Muster vorliegt: Bei fehlenden Werten ist eine ungünstige Variablenausprägung wahrscheinlicher. Daher konnten auch die imputationsbasierten Verfahren hier trotzt ihrer potenziellen Vorteile nicht sinnvoll genutzt werden (Rubin, 1976, 1987, 1996).

Entsprechend musste ein Vorgehen mit unvollständigen Datensätzen gewählt werden. Um möglichst wenig Fälle ausschließen zu müssen, wurde einerseits bei der Skalenbildung für jeden Teilnehmenden ein Mittelwert der Items gebildet, auch wenn nicht alle Items einer Skala beantwortet wurden (Tabachnick \& Fidell, 2014). Da dabei der maximale Anteil der zu ersetzenden Werte einer Skala über alle Teilnehmenden hinweg bei ca. $6 \%$ und durchschnittlich über alle Skalen hinweg nur bei ca. $2 \%$ lag, sind mögliche Verzerrungen durch dieses Vorgehen zu vernachlässigen. Trotz dieser relativ geringen Missingquoten, hätte ein Fallausschluss aufgrund einzelner fehlenden Werte einer Skala die Teststärken gerade bei kleinen Stichproben massiv beeinträchtigt. Weshalb in diesem Fall der Nachteil eines erheblichen Informationsverlustes den möglichen Nachteil von Verzerrungen um ein Vielfaches überwogen hätte.

Andererseits wurde in den jeweiligen Analysen ein listenweiser Fallausschluss gewählt, wobei entsprechend dem Prinzip des paarweisen Fallausschlusses für jede Analyse die

27 Dabei werden fehlende Werte in mehreren Imputationsverfahren durch plausible Werte ersetzt, die regressionsanalytisch aus den vorhandenen Werten vorhergesagt werden. Durch diese mehrfache Simulation, den sogenannten Imputationen, ergeben sich mehrere Datensätze mit unterschiedlich imputierten Werten. So entstehen mehrere komplette Datensätze, die in den statistische Zielanalysen kombiniert genutzt werden (Rubin, 1987). Durch dieses Vorgehen bleibt die Varianz des urspürglichen Datensatzes in den Daten erhalten wodurch statistische Auswertungsverfahren nicht verzerrt werden - wie bei anderen Verfahren zur Generierung vollständiger Datensätze (z. B. Ersetzten der fehlenden Werte durch Skalenmittelwerte der vorhandenen Fälle oder auch der einfachen Imputation). Zudem wird durch die Nutzung mehrerer imputierter Datensätze die Unsicherheit bei der Schätzung der ersetzten Werte berücksichtigt. 
maximal verfügbare Probandenzahl verwendet wurde, die für alle dabei analysierten Variablen mindestens einen Wert angegeben hatten. Zudem wurden Regressionsanalysen, deren Robustheit und Belastbarkeit stärker von der Stichprobengröße abhängt als tTests, zusätzlich mit Bootstrapping-Analysen und Mittelwertsubstitution durchgeführt, um die Ergebnisse an einer teststärkeren Analyse zu überprüfen. Durch diese Vorgehensweisen konnte die Größe von Analysestichproben und somit Teststärken unter den gegebenen Rahmenbedingungen für die jeweiligen Analyseverfahren maximiert werden. Dennoch darf nicht übersehen werden, dass damit das Problem möglicher Verzerrungen aufgrund eines Zusammenhangs zwischen Rücklauf und Prädiktor- sowie Kriteriumsvariablen nicht gelöst ist, da in die Analysen trotzdem nur die Teilstichproben eingehen, die zu den jeweilig betrachteten Messzeitpunkten einen Fragebogen abgegeben bzw. mindestens ein Item der betrachteten Variablen angegeben haben.

\subsubsection{Skalenbildung}

Die verwendeten Skalen wurden vorwiegend selbst formuliert bzw. konstruiert (wie in der Evaluationsforschung gängig), um eine Passung zu Trainings- und Unternehmenskontext zu ermöglichen. Um das daraus resultierende Risiko von Unreliabilität und fraglicher Validität zu minimieren, wurde jedoch versucht möglichst nahe an Aufbau bzw. Formulierung validierter Skalen und Fragebogeninstrumenten zu bleiben ${ }^{28}$. Wie in Abschnitt 5.3.3 ab Seite 110 erläutert, wurden die Skalen durch Mittelwerte der Items gebildet, auch wenn nicht alle Items einer Skala beantwortet wurden (Tabachnick \& Fidell, 2014).

Aufgrund der kleinen Stichprobengröße sind Faktorenanalysen zur Überprüfung der Skalenstruktur der Fragebögen nicht sinnvoll durchführbar. Daher wurden für eine sinnvolle Skalennutzung und -interpretation Skalenoptimierungen durch Eliminierung besonders unreliabler Items anhand von Reliabilitätsanalysen basierend auf Cronbachs $\alpha$, Inter-Item-Korrelationen und Trennschärfen vorgenommen. Auch wenn einige Autoren die Verwendung von Skalen empfehlen, die interne Konsistenzen mit Cronbachs Alpha von $>.8$ aufweisen, weisen sie gleichzeitig darauf hin, dass in einigen Fällen (insbesondere bei Skalen mit wenigen Items, komplexen psychologischen Konstrukten und neu konstruierten Skalen) niedrigere Werte realistischer sind und dennoch ihre inferenzstatistischen Auswertungen belastbare Ergebnisse hervorbringen können (J. M. Cortina, 1993; Hair, 2014; Kline, 1999). Da es sich bei den hier verwendeten Skalen um modifizierte bzw. gänzlich neu konstruierte und selbstformulierte Skalen komplexer Konstrukte han-

${ }^{28}$ Aufgrund von Betriebsratsvorgaben mussten einzelne Formulierungen und Skalen vorab und während der Erhebung noch weiter modifiziert werden. 
delt, die größtenteils auch weniger als 5 Items umfassen, sind interne Konsistenzen unter .8 eher zu erwarten. So wurde für die nachfolgenden Datenanalysen - entgegen der gängigen Konvention, dass eine Skala mit einem Alpha von $>.7$ als reliable gelten kann (Field, 2018) - der in angewandter Forschung häufig genutzte Grenzwert für Cronbachs Alpha von .6 gewählt (Nunnally \& Bernstein, 1994; Pfeiffer, Heslin \& Jones, 1976). Wobei insbesondere bei niedrigen Alphawerten jeweils das Verhältnis zur Itemzahl, Trennschärfe und Inter-Item-Korrelationen berücksichtigt wurde, um im Einzelfall zu entscheiden, ob eine ausreichende interne Konsistenz für eine Skalennutzung vorliegt (J. M. Cortina, 1993; Field, 2018). Bei Skalen mit Subskalenstruktur wurden gemäß Cronbach (1951) auch die internen Konsistenzen der Subskalen auf diese Grenzwerte hin überprüft.

Zudem wurden aufgrund erhebungsökonomischer Aspekte auch Single-Item-Skalen verwendet (z. B. direkte Veränderungsmessungen oder Transfermöglichkeit). Bei AnaIysen von Variablen mit geringer Skalengüte wurden die Ergebnisse zusätzlich mit Single-Items überprüft (entsprechende Auswahl siehe Tabelle 6 ab Seite 118 für Skalen der Transferdeterminanten und Tabelle 7 ab Seite 127 für Skalen des Trainings- und Transfererfolgs). Dabei wurden Handlungsempfehlungen für den Einsatz von Single-Item Measures berücksichtigt, die in Fisher, Matthews und Gibbons (2016) zusammengefasst sind. Unter dem Aspekt des maximal möglichen Erkenntnisgewinns unter gegebenen Rahmenbedingungen des Feldes bzw. Forschungsvorhabens, wird dieses Vorgehen als sinnvoll betrachtet.

Alle Fragebogenitems wurden auf einer fünfstufigen Likert-Skala von 1 („trifft gar nicht zu“) bis 5 („trifft völlig zu“) gemessen. Einzige Ausnahme bilden die direkten Veränderungsmessungen, die zwar ebenfalls fünfstufig erfasst wurden, jedoch auf einer Skala von -2 („deutlich verschlechtert“) bis +2 („deutlich verbessert“) abgefragt wurden. Für die Auswertungen wurden auch diese Items in die Likert-Skala von 1 („deutlich verschlechtert“) bis 5 („deutlich verbessert") transformiert. Zudem wurden invertierte Items bzw. Skalen mit den Skalenstufen 1 („trifft völlig zu“) bis 5 („trifft gar nicht zu“) umgekehrt, um für alle Variablen ein einheitliches Format von 1 (ungünstigste Ausprägung) bis 5 (günstigste Ausprägung) zu erhalten. 


\subsubsection{Skalenbeschreibung}

Wie aus der Trainings- und Transferforschung abgeleitet (siehe Abschnitt 3.4 ab Seite 83), wurden im vorliegenden Forschungsprojekt im Sinne eines ganzheitlichen Ansatzes die identifizierten Transferdeterminanten des GLTSI-Modells (Bates et al., 2008; Kauffeld et al., 2008) um die weiteren relevanten Variablen Lern- und Trainingsmotivation sowie um multidimensionale Variablen zum Trainings- und Transfererfolg nach Kirkpatrick (1967, 1998) ergänzt. Das daraus resultierende Studienmodell mit den erfassten Variablen ist in Abbildung 12 auf Seite 87 zusammengefasst. Mit welchen Intems und zu welchen Messzeitpunkten die einzelnen Variablen erfasst wurden, wird nach einem Überblick über die berücksichtigten Kontrollvariablen für Transferdeterminanten sowie für Trainings- und Transfererfolg beschrieben.

\section{Kontrollvariablen}

Basierend auf bisheriger Forschung zum Trainings- und Transfererfolg wurden die folgenden demographischen Variablen als möglicherweise relevant identifiziert und erfasst (Blume et al., 2010; Lacerenza et al., 2017): Geschlecht (1 = männlich, 2 = weiblich), Alter (in Jahren), Bildungsabschluss ( $0=$ Ausbildung, $1=$ Studium), Führungserfahrung (in Monaten), Hierarchiestufe ( $1=$ oberstes Management/Vorstand bis $5=$ erste Führungsstufe/Teamleitung) und Bereich ( 0 = Produktion, $1=$ Verwaltung). In den jeweiligen Analysen wurden nur die Kontrollvariablen berücksichtigt, die einen tatsächlichen Zusammenhang mit den jeweiligen Kriteriumsvariablen zeigten. Zudem wurden Teilnehmendeneigenschaften durch Verwendung von abhängigen t-Tests und Ausgangswerten von Prä-Post-Variablen kontrolliert. Eigenschaften von Training und Arbeitsumgebung wurden durch Konstanthaltung kontrolliert, da diese als konfundierende Einflussfaktoren für die betrachteten abhängigen Variablen gelten (Berardinelli, Burrow \& Dillon Jones, 1995; Egan, 2008; Holton, Hsin-Chih \& Naquin, 2003; Russ-Eft, Dickison \& Levine, 2010). 


\section{Transferdeterminanten}

Die Auswahl und Kategorisierung der Transferdeterminanten basierte auf der von Kauffeld et al. (2008) vorgeschlagenen GLTSI-Struktur zur umfassenden Erhebung relevanter Transferfaktoren (siehe Abbildung 10 auf Seite 74). Auch wenn nicht alle Transferfaktoren des GLTSI im Forschungsfokus liegen und Kauffeld et al. (2008) deren Transferrelevanz nur teilweise bestätigen konnten, wurden sie komplett miterhoben, da sie möglicherweise für die Erklärung der Ergebnisse der Hypothesenprüfung hilfreich sind. Denn das vorrangige Ziel der Untersuchungen liegt gerade im Erkenntnisgewinn bezüglich der Transfermechanismen. Eine generelle Abweichung von der GLTSI-Struktur bestand schließlich in der Einordnung der Transferdeterminanten in das bewährte und am häufigsten verwendete Transfermodell nach Baldwin \& Ford (1988; siehe Abschnitt 3.2.2 ab Seite 72 sowie Abbildung 12 auf Seite 87 ) ergänzt um die bestätigten Transferdeterminanten Lern- und Trainingsmotivation sowie den noch wenig untersuchten Faktor Selbstwertschätzung für explorative Analysen:

\section{Teilnehmendeneigenschaften}

1) Selbstwertschätzung

2) Lernmotivation

3) Trainingsmotivation

4) Selbstwirksamkeitserwartung

5) Leistungserwartung

6) Ergebniserwartung

7) Erwartungsklarheit (trainingsspezifisch)

8) Transfermotivation (trainingsspezifisch)

\section{Trainingsgestaltung}

1) Inhaltsrelevanz (trainingsspezifisch)

2) Transferdesign (trainingsspezifisch)

\section{Arbeitsumfeld}

1) Kollegenunterstützung

2) Vorgesetztenunterstützung

3) Offenheit für Änderungen in Gruppe

4) Feedback

5) Negative Folgen bei Nicht-Anwendung

6) Positive Folgen bei Anwendung (trainingsspezifisch)

7) Sanktionen durch Vorgesetzte (trainingsspezifisch)

8) Transferkapazität (trainingsspezifisch)

9) Anwendungsmöglichkeit (trainingsspezifisch) 
Die GLTSI-Unterscheidung zwischen generell bzw. trainingsübergreifend und trainingsspezifisch wirkenden Determinanten wurde für die Überprüfung der Transfermechanismen übernommen. Diese Einteilung gibt zugleich vor, ob Faktoren nur einmalig für die gesamte Trainingsreihe oder mehrfach, für jeden Baustein spezifisch durch zusätzliche Befragungen vor bzw. unmittelbar nach den jeweiligen Trainingsbausteinen erhoben wurden. Entgegen der von Kauffeld et al. (2008) vorgeschlagenen Einteilung, wurden jedoch drei der elf trainingsspezifischen Faktoren im Kontext dieses Forschungsprojektes den generellen Faktoren zugeordnet. Diese Modifikation der Zuordnung liegt in der Annahme begründet, dass die Skalen „Kollegenunterstützung“, „Vorgesetztenunterstützung" und "Negative Folgen bei Nicht-Anwendung“ in der hier betrachteten Trainingsreihe über die drei Trainingsbausteine hinweg keine größeren Unterschiede aufweisen sollten.

Die GLTSI-Klassifikation von Selbstwirksamkeitserwartung und Erwartungsklarheit als sekundäre Teilnehmendeneigenschaften ${ }^{29}$ wurde insofern berücksichtigt, dass diese Skalen bei der Überprüfung eines Alternativmodells zur Erklärung von Transfer durch primäre Transferdeterminanten (Hypothese 2) in der übergeordneten Skala transferförderlicher Teilnehmendeneigenschaften nicht einbezogen wurden. Bei der Erklärung primärer Transferdeterminanten (Hypothese 3) wurden sie dagegen berücksichtigt.

Eine weitere Abweichung von der GLTSI-Struktur besteht in der bereits genannten Ergänzung der generellen Transferfaktoren, durch „Lernmotivation“ und „Trainingsmotivation“ basierend auf den Erkenntnissen von Noe und Wilk (1993) sowie der Erfassung unterschiedlicher Selbstwirksamkeitsfacetten (siehe Abschnitt 3.2.3 ab Seite 76 und Abschnitt 4 ab Seite 85). Während der GLTSI Selbstwirksamkeit auf den Trainingstransfer ausrichtet, wird für dieses Forschungsprojekt die Skala aus Items zur generellen Selbstwirksamkeitserwartung nach Schwarzer und Jerusalem (1999), zur beruflichen Selbstwirksamkeitserwartung nach Schyns und Collani (2002) sowie zur transferbezogenen Selbstwirksamkeitserwartung gemäß GLTSI zusammengestellt. Als weiterer möglicher aber noch kaum beachteter Transferfaktor wird darüber hinaus Selbstwertschätzung nach Rosenberg $(1965,1989)$ bzw. Collani und Herzberg (2003) miterfasst, um explorativ mögliche Transfereinflüsse untersuchen zu können.

So wurden insgesamt elf generelle Transferfaktoren übergeordnet in einer Vorbefragung erfasst. Weitere acht trainingsspezifische Faktoren wurden für jeden Baustein einzeln

${ }^{29}$ Gemäß GLTSI wirken Selbstwirksamkeitserwartung und Erwartungsklarheit nur indirekt auf Transfer vermittelt durch motivationale Transferdeterminanten (siehe Abbildung 10 auf Seite 77). 
erhoben. Diese können nochmals unterteilt werden in Faktoren, die bereits vor den Trainings eingeschätzt werden können und welche, die erst nach der Trainingsteilnahme sinnvoll bewertet werden können. Zu Letzteren gehören die Transferfaktoren „Transferdesign“, „Inhaltsrelevanz“ und „Anwendungsmöglichkeit“. Die übrigen lassen sich zumindest als Erwartungen basierend auf der Vorinformation aussagekräftig beantworten. Zudem eignen sie sich zur Vorbereitungsreflexion auf die einzelnen Bausteine und können damit gleichzeitig der Transferförderung dienen.

Da die primäre Transferdeterminante "Transfermotivation" in der Trainingsforschung als besonders bedeutend erachtet wird (siehe Abschnitt 3.2.1 ab Seite 45) und aufgrund von Forschungserkenntnissen davon ausgegangen werden kann, dass transferförderliche Trainings die Transfermotivation steigern können (Gegenfurtner et al., 2009), wurde diese Variable zusammen mit dem möglicherweise antezedenten Faktor „Positive Folgen bei Anwendung" (siehe Erwartungs-x-Wert-Theorien) sowohl vor als auch unmittelbar nach den Bausteinen erhoben. Bei der „Transfermöglichkeit“ als situative Komponente der primären Transferdeterminanten ist entscheidend, wie sie über die betrachtete Transferzeit hinweg tatsächlich ausgeprägt war (da dies nur bedingt im Einflussbereich der einzelnen Teilnehmenden liegt. Daher wurde diese Transferdeterminante retrospektiv zu T3 durch ein Single-Item der Skala Transferkapazität erfasst („Mein Tagesablauf erlaubt es mir, Dinge zu ändern, um meine neuen Kenntnisse einbringen zu können“; N $=40, M=3.16, S D=0.84)$. Die Operationalisierung der zentralen primären Transferdeterminante „Kompetenz“ wird gemäß der Kategorisierung nach Kirkpatrick $(1967,1998)$ im nächsten Abschnitt zur Operationalisierung von Trainings- und Transferfolg erläutert (Unterabschnitt Lernen bzw. Kompetenzniveau ab Seite 132).

Die Skalen der Transferdeterminanten mit Beschreibung, Items, Messzeitpunkten, Deskriptiva und Angaben zur internen Konsistenz sind in Tabelle 6 ab Seite 118 zusammengefasst. Die im Rahmen der Skalenoptimierungen eliminierten Items sind dort ebenfalls abgebildet jedoch durchgestrichen markiert. Daraus geht hervor, dass die Skalengüte zumindest nach Skalenoptimierungen trotz Stichprobengröße und selbstkonstruierter Skalen mit wenigen Items vorwiegend als akzeptabel bis sehr gut bewertet werden konnte. 


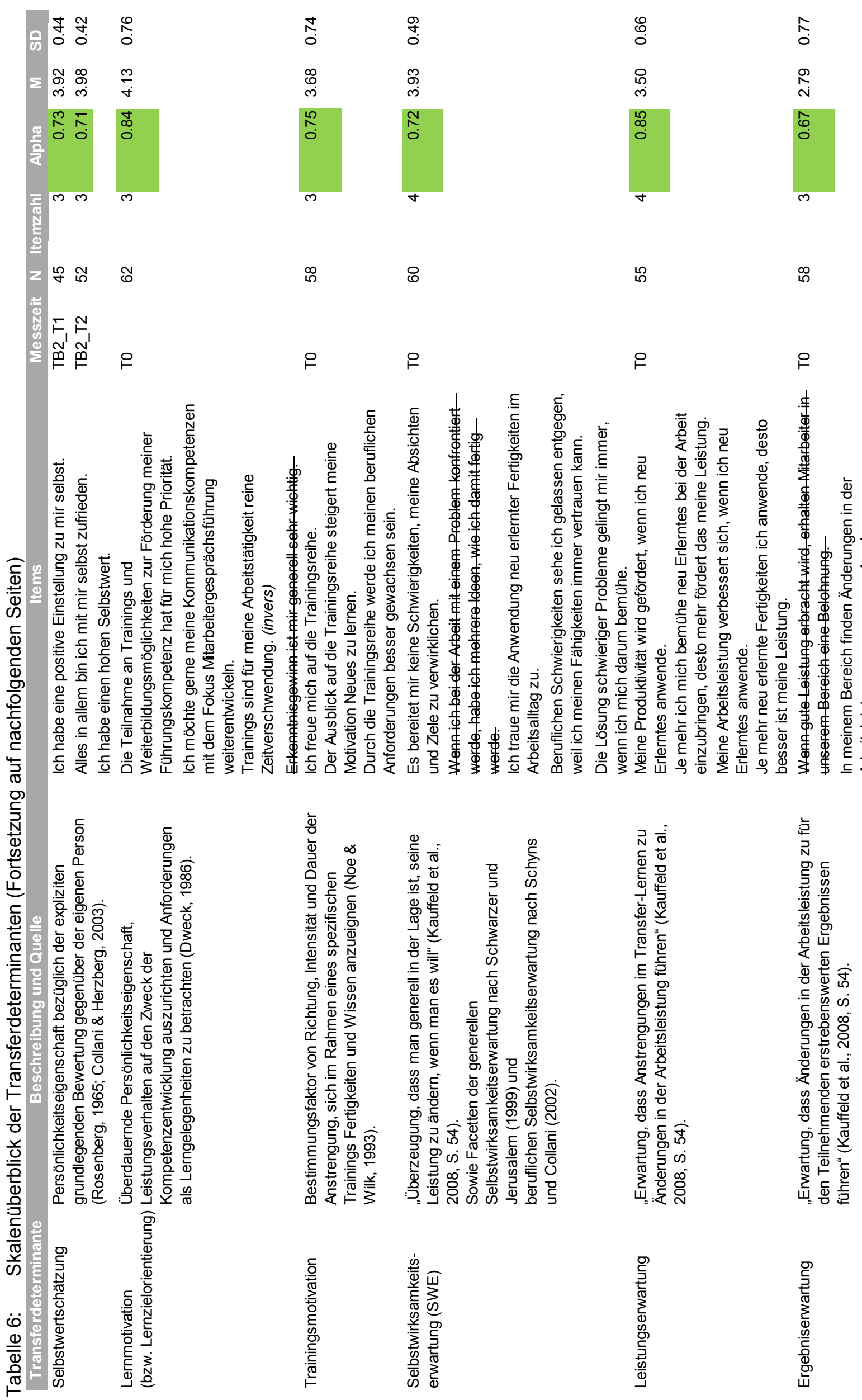




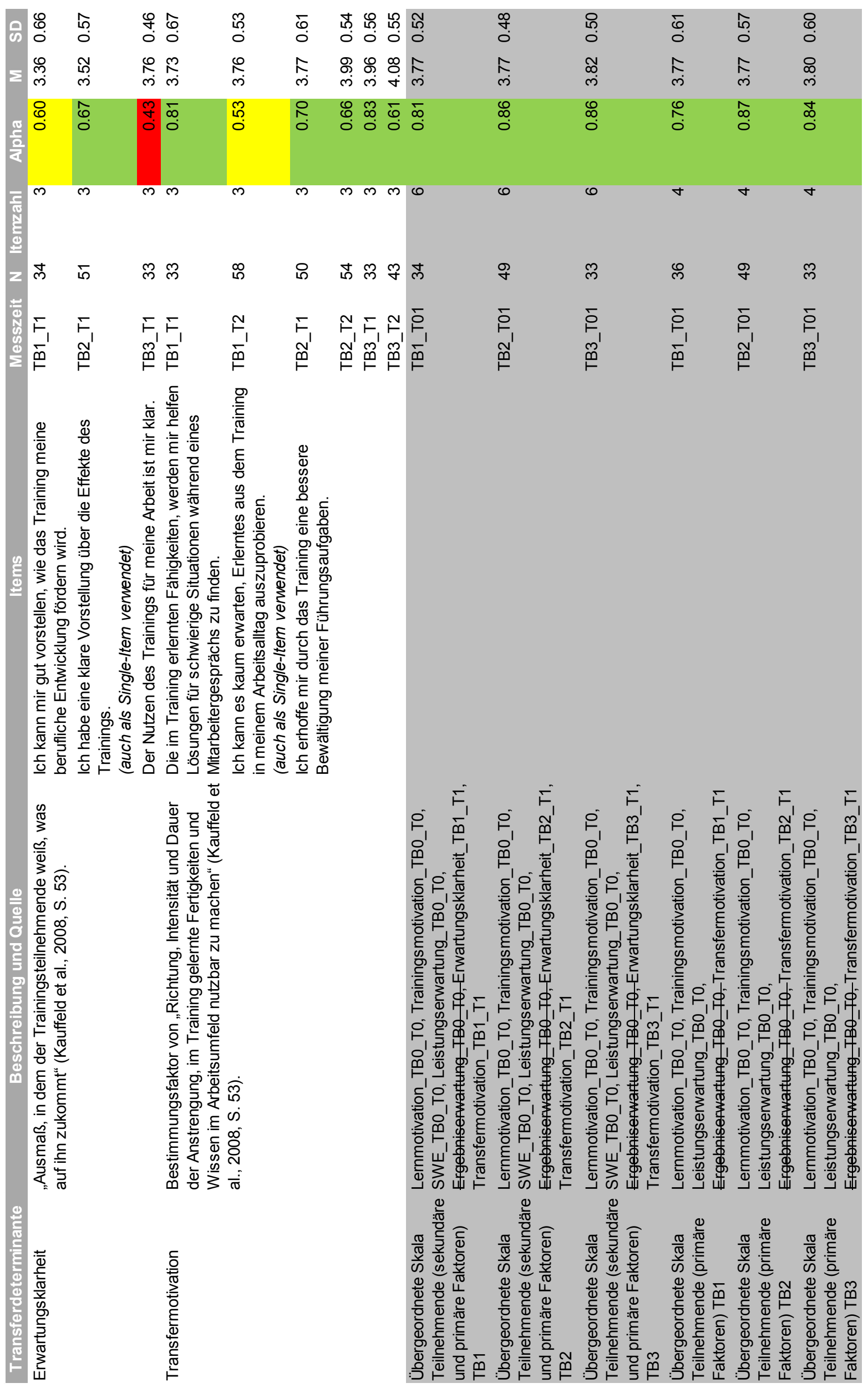



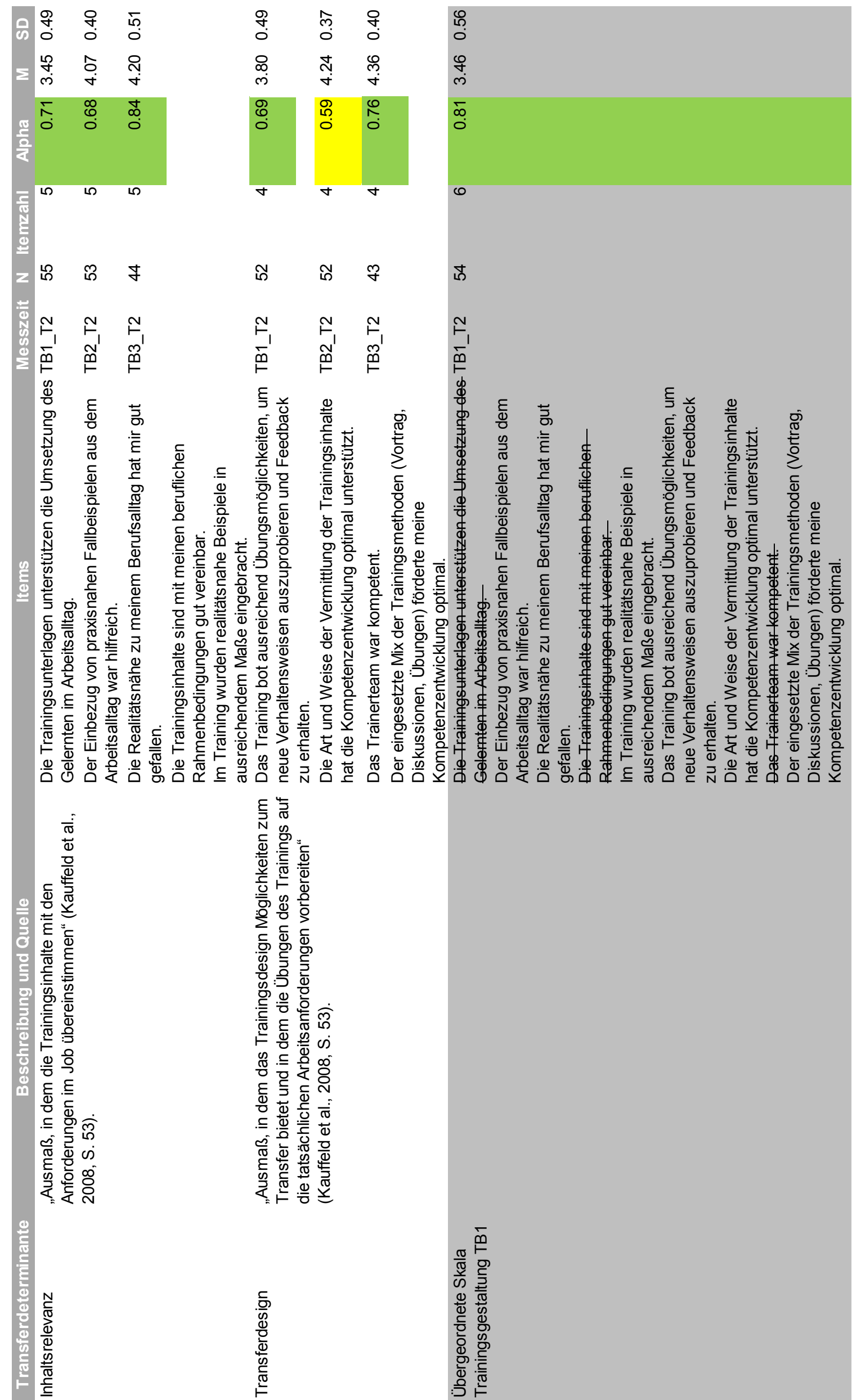


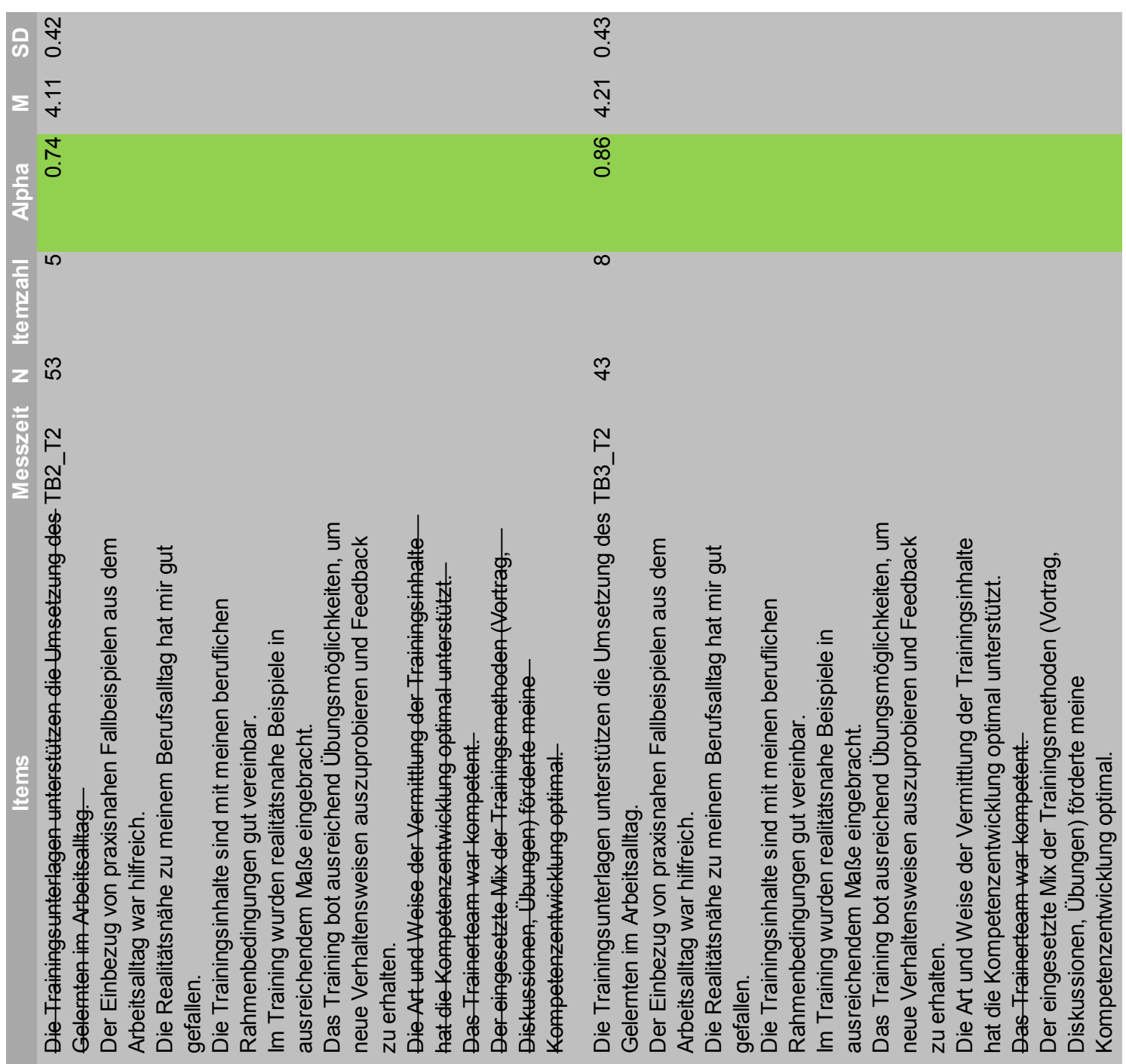




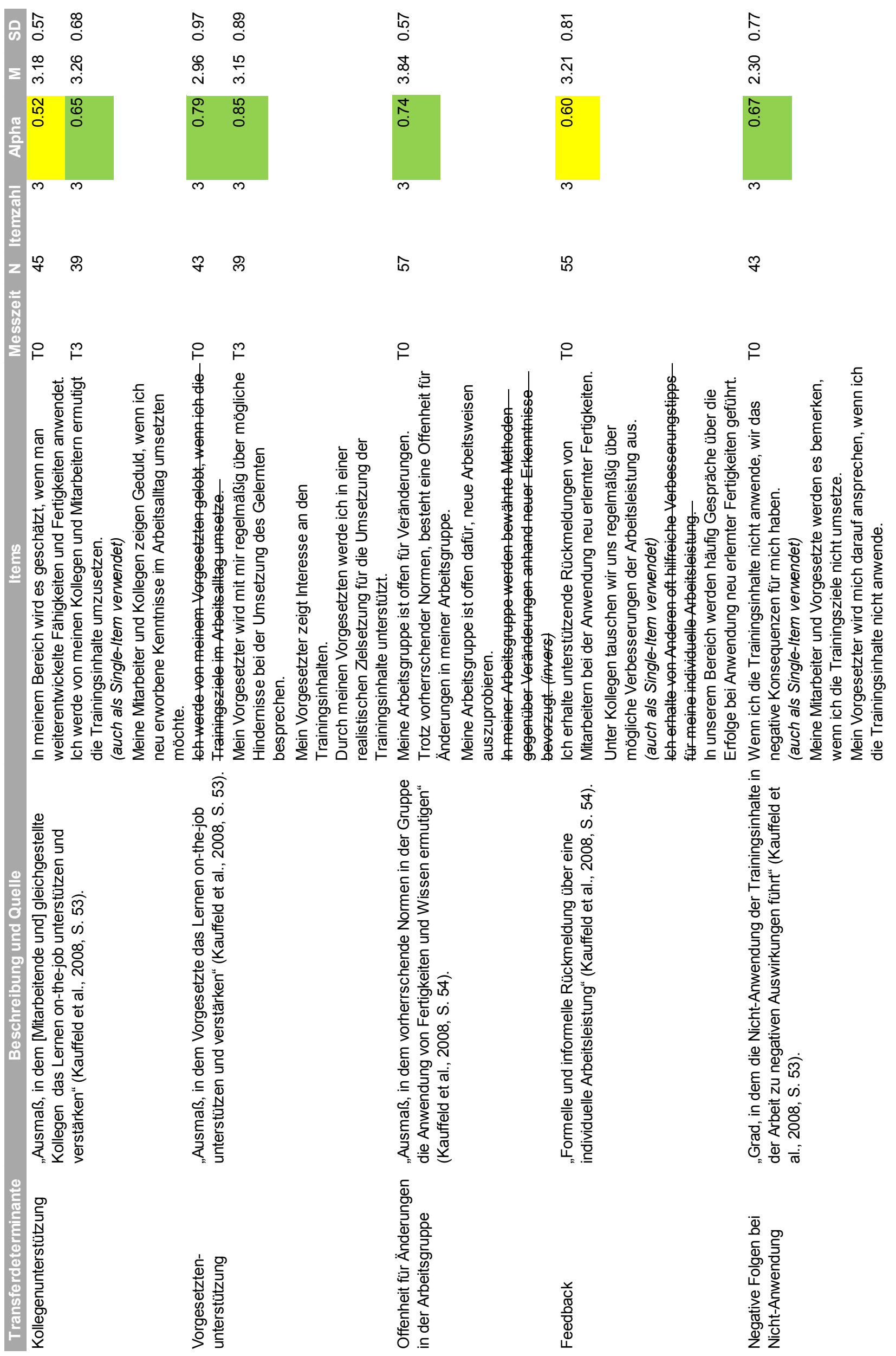



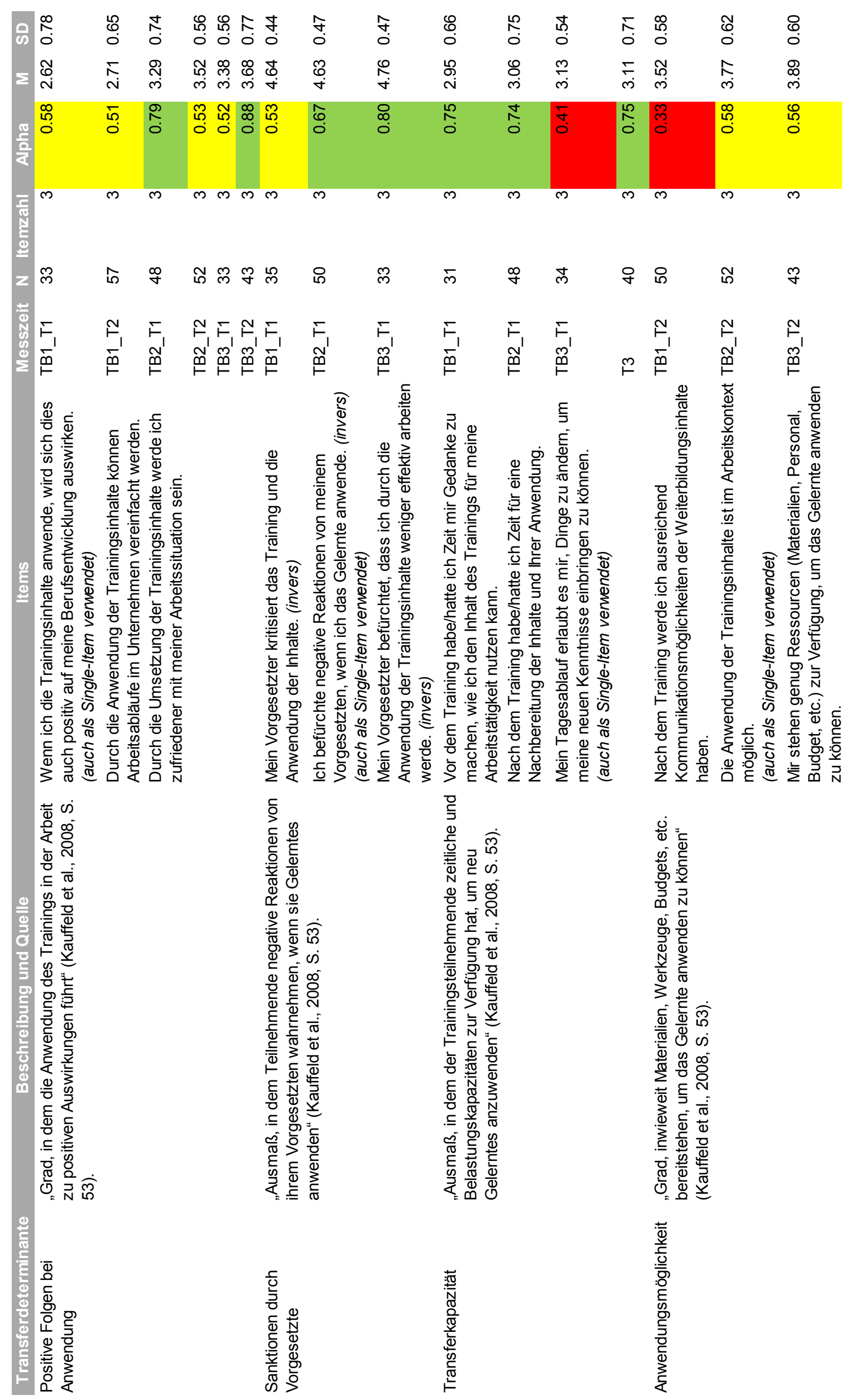


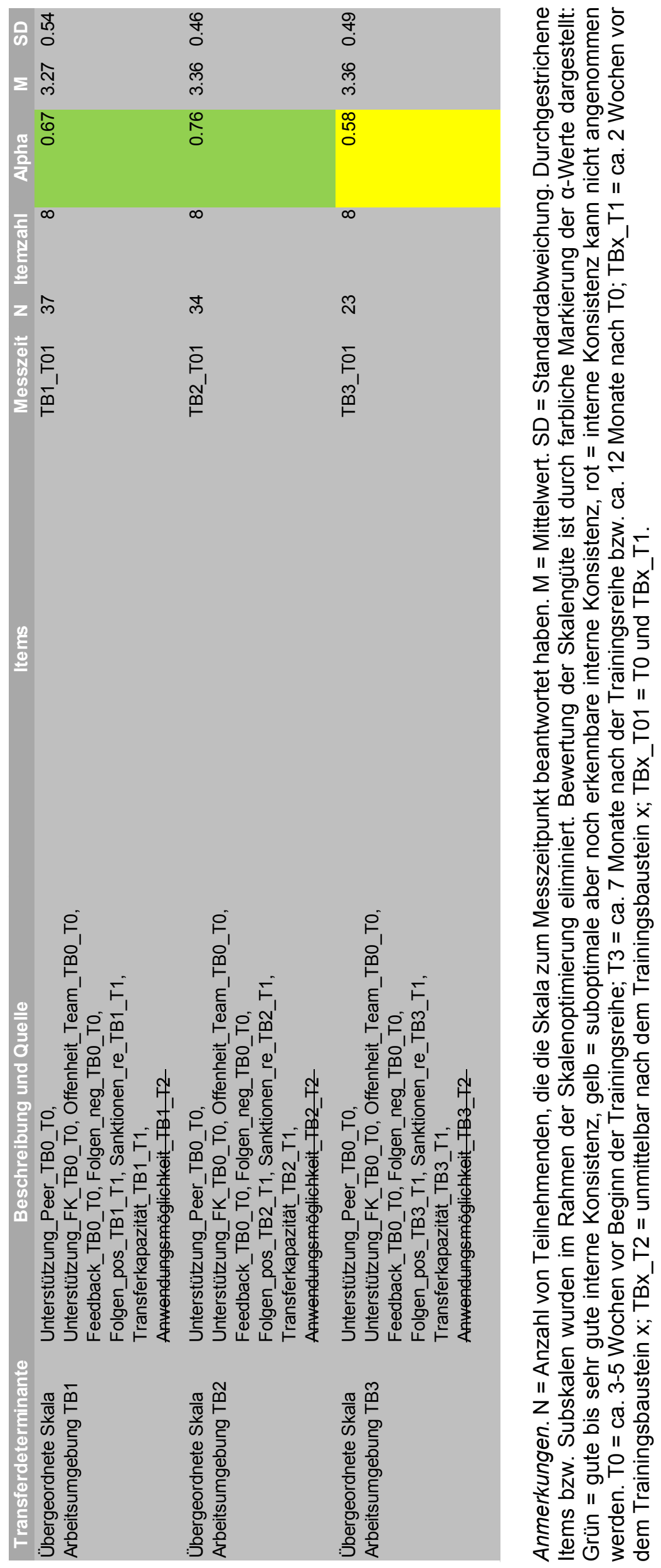


Für die Teilnehmendeneigenschaften zeigte nur Erwartungsklarheit vor Trainingsbaustein 3 keine interne Konsistenz. Die übrigen Subskalen hatten Cronbacha-Werte zwischen .53 und .85, wovon lediglich eine weitere Skala unter dem gängigen Grenzwert von .6 lag. Davon zeigten die Skalen „Erwartungsklarheit“ vor sowie "Transfermotivation“ nach dem Trainingsbaustein 1 suboptimale Skalengüte. Daher sollten sie nur mit Vorsicht in die Interpretation einbezogen werden, da eine itemübergreifende Schlussfolgerung nicht für alle Konstruktfacetten repräsentativ sein könnte und ein ß-Fehler warscheinlicher ist. Die übergerondeten Skalen der Teilnehmendeneigenschaften sowohl mit als auch ohne sekundäre Transferdeterminanten zeigten alle gute bis sehr gute interne Konsistenzen mit einem Cronbachs $\alpha$ zwischen .76 und .87. Allerdings konnten diese Skalengüten erst erreicht werden nachdem die Subskala „Ergebniserwartung“ eliminiert wurde. Insofern konnten bei den Analysen mit den übergeordneten Skalen, die GLTSI-Skalen nicht mehr vollständig berücksichtigt werden.

Die Subskalen der Trainingseigenschaften zeigten ein Cronbachs $\alpha$ zwischen .59 und 84. Dabei zeigte nur die Skala „Trainingsdesign“ nach Trainingsbaustein 2 suboptimale Skalengüte und sollte mit Vorsicht interpretiert werden. Da in die übergeordneten Skalen der Trainigsgestaltung nur zwei Subskalen einfließen, wurden diese basierend auf den zugeordneten Items gebildet und optimiert. Die resultierenden Cronbach- $\alpha$-Werte lagen zwischen .74 und .86. Um in diesen guten Bereich zu gelangen, war es jedoch nötig bei den verschiedenen Trainingsbausteinen unterschiedliche Items zu eliminieren. Um zwischen den Bausteinen vergleichen zu können, sollte daher auf Subskalenniveau analysiert werden.

Bei den Subskalen der Arbeitsumgebung waren die Skalengüten sehr durchmischt und

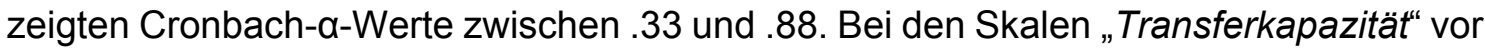
Trainingsbaustein 3 und erwartete „Anwendungsmöglichkeit" nach Trainingsbaustein 1 konnte eine interne Konsistenz nicht angenommen werden. Daher können für diese beiden Trainingsbausteine das Gesamtmodell bzw. diese Subskalen nicht sinnvoll analysiert werden. Darüber hinaus zeigten einige Subskalen der Arbeitsumgebung suboptimale Skalengüte: „Kollegenunterstützung“ und "Feedback“ vor der Trainingsreihe, „Positive Folgen bei Anwendung" vor und nach Trainingsbaustein 1, nach Trainingsbaustein 2 und vor Trainingsbausten 3, „Sanktionen durch Vorgesetzte“ (invertiert) vor Trainingsbaustein 1 sowie „Anwendungsmöglichkeit“ nach Trainingsbaustein 2 und 3 . Die übergeordenten Skalen zeigten Cronbach- $\alpha-W e r t e$ zwischen .58 und .76, allerdings erst nach Eliminierung der Subskala erwartete Anwendungsmöglichkeit unmittelbar nach den drei Trainingsbausteinen. Eine Erklärung könnte sein, dass transferförderliche Trainings die Wahrnehmung der Teilnehmenden 
bezüglich Transfermöglichkeiten positiv beeinflussen, während die übrigen Arbeitsumgebungsfaktoren vorwiegend von Rahmenbedingungen und Interaktionspartnern abhängen. Allerdings war trotz Skalenoptimierng die interne Konsistenz der übergeordenten Skala Arbeitsumgebung für TB3 suboptimal und sollte aufgrund der nicht vorhandenen Konsistenz ihrer Subskala Transferkapazität für Analysen nicht verwendet werden.

\section{Trainings- und Transfererfolg}

Die Auswahl und Kategorisierung der Variablen von Trainings- und Transfererfolg orientierte sich übergeordnet an dem Basismodell der Wirksamkeitsanalyse nach Kirkpatrick (siehe Abschnitt 2.2 ab Seite 12) und wird daher in den nachfolgenden Abschnitten für die vier Ebenen Reaktion, Lernen, Transfer und Resultate beschrieben. Entsprechend der Forschungsfragen liegt dabei der Fokus auf der Kriteriumsebene des Transfers. Dennoch wird versucht die anderen Ebenen insofern mit zu erfassen, wie sie für die Erklärung der Transferwirkungen von Bedeutung sind.

Zur Erfassung des Trainingserfolges wurde die Reaktion unmittelbar nach den jeweiligen Trainingsbausteinen sowie das Kompetenzniveau bezüglich Trainingsinhalte (vor und unmittelbar nach den jeweiligen Trainingsbausteinen) abgefragt. Zur Erfassung des Transfererfolges wurden entsprechend der Implikationen bisheriger Transferforschung bezüglich einer präziseren und differenzierten Operationalisierung (Ford et al., 2018; Gagne, 1965; Laker, 1990) „naher Transfer“ (vor den jeweiligen Trainingsbausteinen und zu T3), „weiter Transfer“ (zu T0 und T3) und resultierende Verbesserung des Führungsverhaltens (zu T3 mit zusätzlichen Mitarbeitendeneinschätzungen) abgefragt. T3 wurde ca. 7 Monate nach dem letzten Trainingsbaustein erfasst. Dieser Zeitraum wurde gewählt, um einerseits eine ausreichende Transferzeit zu ermöglichen (Arthur et al., 2003), aber andererseits auch noch nahe genug an den Maßnahmeneffekten zu bleiben und nicht zu viele Konfundierungen durch andere Einflüsse (z. B. weitere Trainings oder organisationale Veränderungen) in Kauf nehmen zu müssen.

Die Skalen des Trainings- und Transfererfolgs mit Beschreibung, Items, Messzeitpunkten, Deskriptiva und Angaben zur internen Konsistenz sind in Tabelle 7 ab Seite 127 zusammengefasst. Die im Rahmen der Skalenoptimierungen eliminierten Items sind dort ebenfalls abgebildet jedoch durchgestrichen markiert. Daraus geht hervor, dass die Skalengüte zumindest nach Skalenoptimierungen trotz Stichprobengröße und selbstkonstruierter Skalen mit wenigen Items vorwiegend als akzeptabel bis sehr gut bewertet werden konnte. 


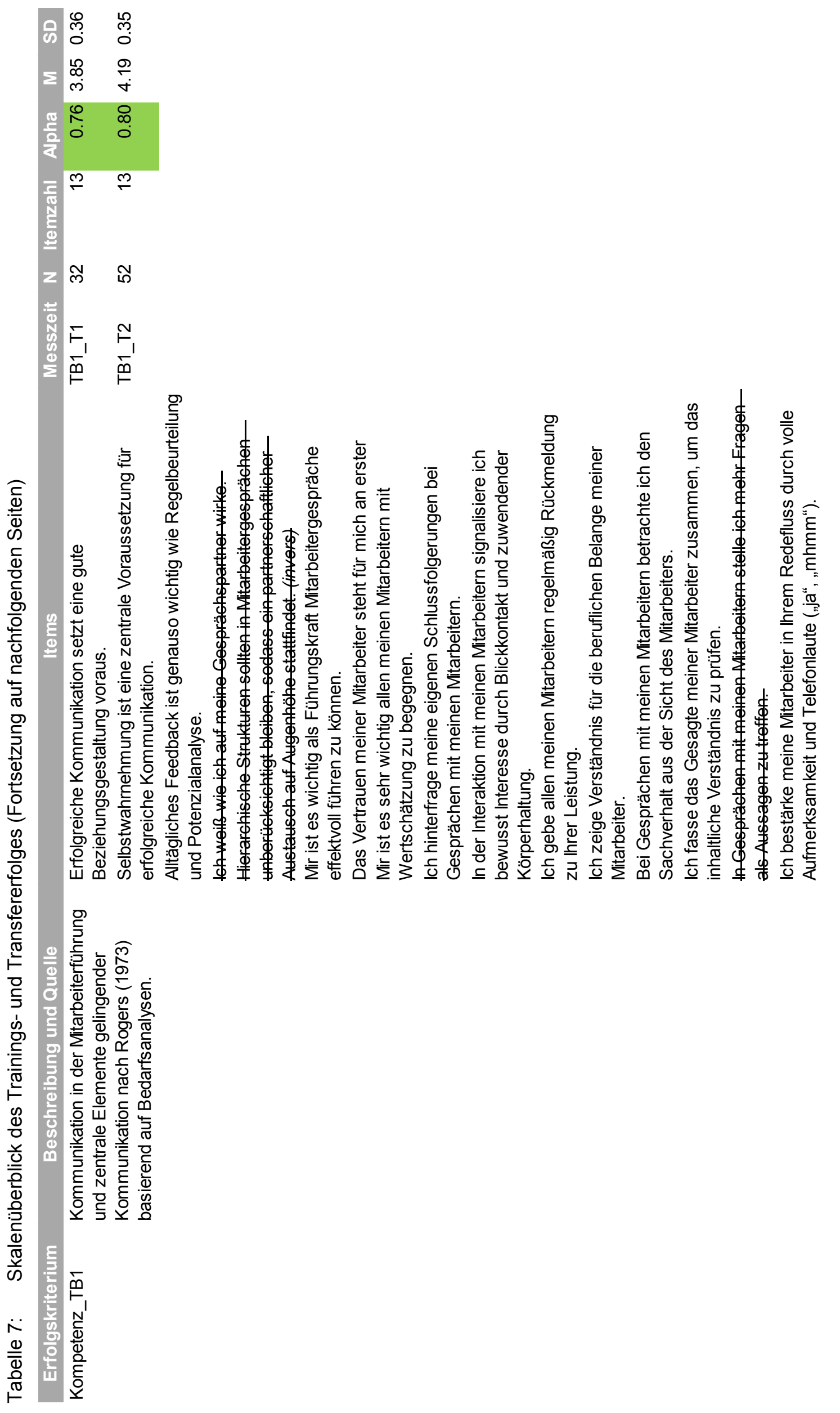




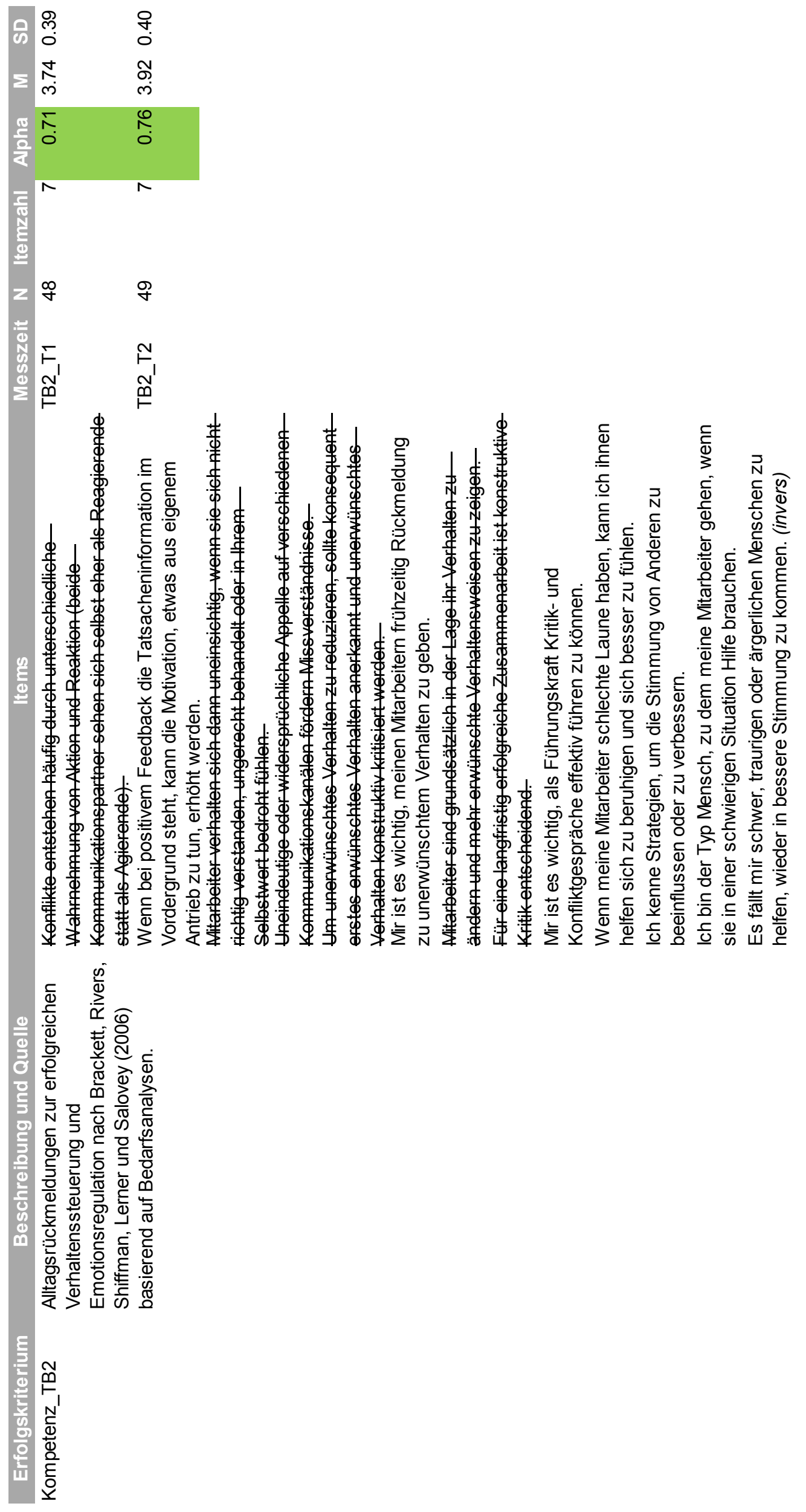




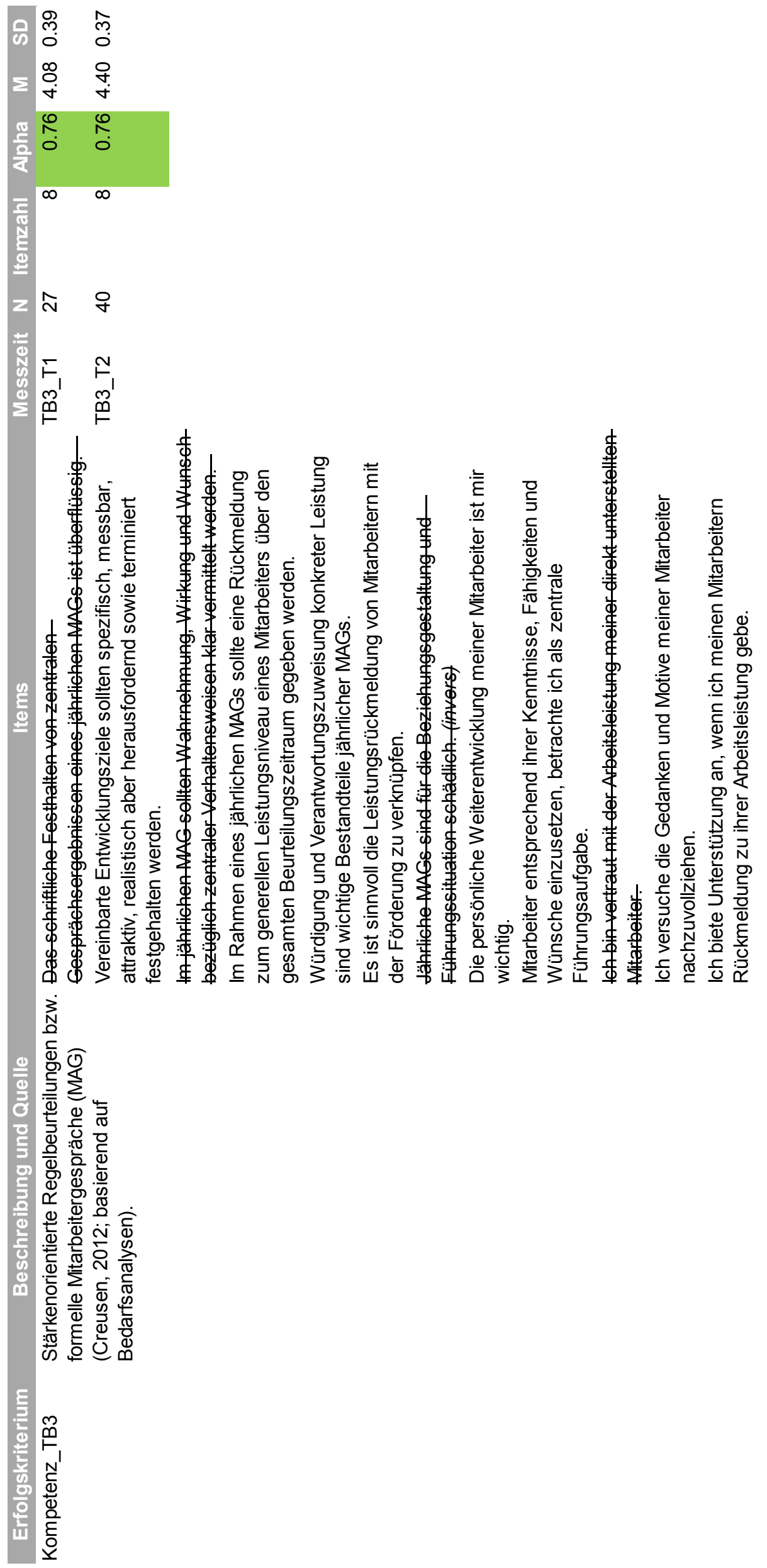




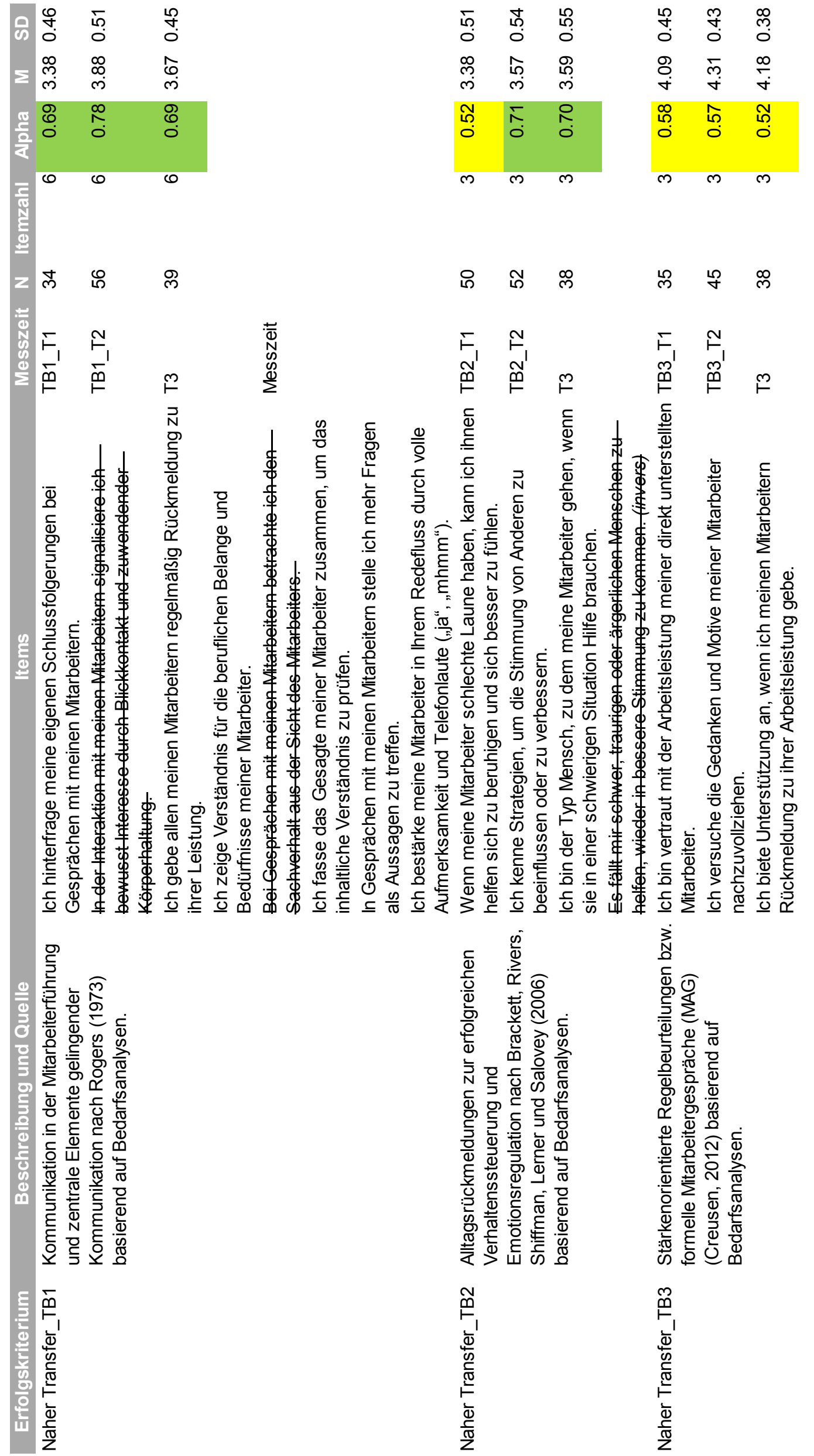




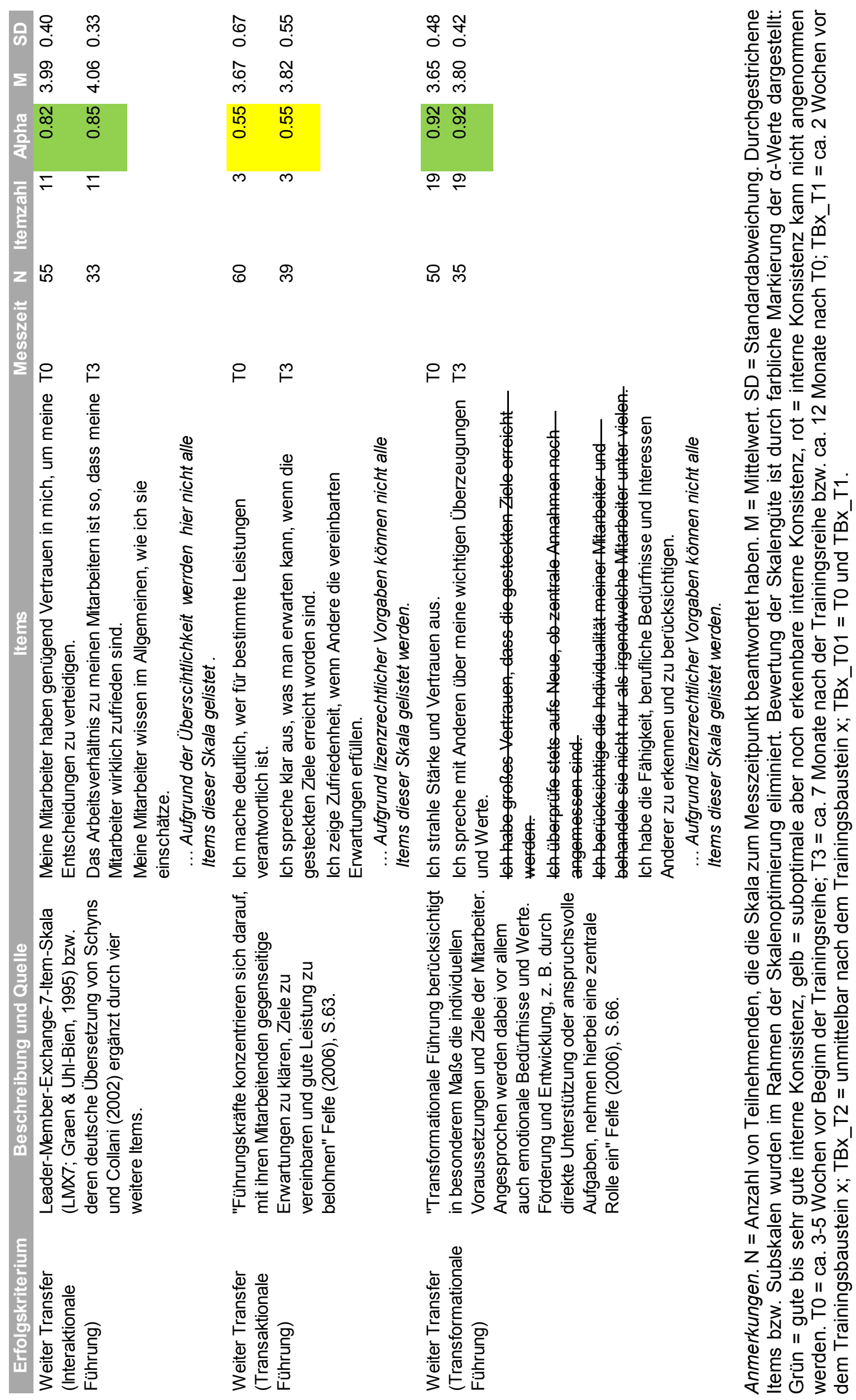




\section{Reaktion}

Für die Reaktionsebene dienten die Bewertungen der Trainingsnützlichkeit basierend auf den retrospektiven Skalen „Transferdesign“ und „Inhaltsrelevanz" (Cronbachs a zwischen .53 und .83) sowie die Veränderungsmessung der Skala „Transfermotivation ${ }^{30 “ " ~}$ (Cronbachs a zwischen .53 und .83), welche bereits im vorigen Abschnitt der Transferdeterminanten ab Seite 115 beschrieben wurden. Lediglich die Skalen „Transferdesign“ nach Trainingsbaustein 2 und "Transfermotivation" nach Trainingsbaustein 1 zeigten suboptimale interne Konsistenzen und sollten entsprechend vorsichtig interpretiert werden (siehe Tabelle 6 ab Seite 118).

\section{Lernen bzw. Kompetenzniveau}

Wie aus der Definition von Führungskräftetrainings nach Felfe und Franke (2014) ersichtlich (siehe Abschnitt 2.1 auf Seite 7), zielte die Trainingsreihe nicht nur auf die Wissensvermittlung, sondern auch auf verhaltenssteuernde Einstellungsveränderung und Erweiterung des Verhaltensrepertoires ab. Entsprechend enthielten die Trainingsbausteine einen Methodenmix aus Didaktik zur Wissensvermittlung, kognitiven Ansätzen zur Einstellungsveränderung und Verhaltensmodellierung zur Erprobung neuer Verhaltensweisen im geschützten Rahmen (siehe Abschnitt 5.2.2 ab Seite 94). So setzte sich die Operationalisierung der Lernebene zur Messung des Trainingserfolgs aus den drei Facetten Wissens-, Einstellungs- \& Verhaltensänderung während des Trainings zusammen. Auf diesem strukturellen Rahmen wurden Skalen entsprechend der jeweiligen Trainingsinhalte selbstkonstruiert. Für den ersten Trainingsbaustein dienten Aspekte erfolgreicher Kommunikation in der Mitarbeiterführung und zentrale Elemente gelingender Kommunikation nach Rogers (1973) als Grundlage. Items des Trainingsbausteins 2 bezogen sich auf Alltagsrückmeldungen zur erfolgreichen Verhaltenssteuerung, wobei die Items zu Verhaltensweisen auf einem bewährten Testverfahren zur Erfassung interaktionaler Emotionsregulation, der Self-Rated Emotional Intelligence Scale (SREIS) nach Brackett, Rivers, Shiffman, Lerner und Salovey (2006), basierten. Items für den dritten Baustein orientierten sich an stärkenorientierte Regelbeurteilungen bzw. formellen Mitarbeitergesprächen (MAG) nach Creusen (2012). Auch diese Items wurden modifiziert, um sie auf den Führungs- und Unternehmenskontext auszurichten. Dennoch zeigten die Skalen des Kompetenzniveaus vor und unmittelbar nach den drei Trainingsbausteinen

${ }^{30} \mathrm{Da}$ die Trainingsforschung darauf hinweist, dass Transfermotivation nach Trainings als Indikator für die Reaktionsebene gelten kann (siehe Abschnitt 3.2.1 ab Seite 44 oder Z. B. Bauer et al., 2016; Alvelos et al., 2015; Bhatti et al., 2014; Grohmann et al., 2014; Seiberling \& Kauffeld, 2017). 


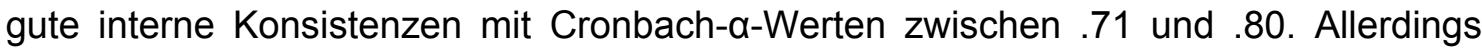
mussten für akzeptable Skalengüte bei Trainingsbaustein 2 knapp die Hälfte der Skalenitems eliminiert werden.

Berücksichtig werden muss, dass die Verhaltensfacette des Kompetenzzuwachses während des Trainings eher als Verhaltensabsicht verstanden werden kann, da Verhaltensänderungen unmittelbar nach dem Training nur bedingt stattfinden und abgefragt werden können. Um tatsächliche Verhaltensänderungen im Arbeitskontext zu erfassen wurden Items der Verhaltensfacette bzw. Zielverhaltensweisen als "naher Transfer" nach einer angemessenen Transferphase mit ausreichend zeitlichem Abstand erneut abgefragt. Dies ist jedoch Gegenstand des Transferverhaltens bzw. der dritten Ebene nach Kirkpatrick (1967, 1998), die im nächstem Abschnitt beschrieben ist.

\section{Transferverhalten}

Zur Erfassung des „nahen Transfers“ wurden die Items zu Verhaltensweisen der eben beschriebenen Kompetenzskalen pro Trainingsbaustein verwendet. Entsprechend lagen hierfür jeweils drei Messzeitpunkte vor (vor und unmittelbar nach den jeweiligen Trainingsbausteinen sowie ca. 7 Monate nach der Trainingsreihe). Die internen Konsistenzen lagen bei Cronbach- $\alpha-W e r t e n$ zwischen .52 und .78. Auch wenn damit vorwiegend akzeptable Skalengüte vorlag, muss bei Analysen mit den Skalen zur Erfassung des nahen Transfers vor Trainingsbaustein 2 und zu allen Messzeitpunkten des dritten Trainingsbausteines berücksichtigt werden, dass vorhandene Effekte möglicherweise nicht signifikant werden.

Darüber hinaus wurden für die Operationalisierung des „weiten Transfers“ Führungsverhaltensweisen erfasst, die nachweislich Leistungsfähigkeit, Motivation und Wohlbefinden der Mitarbeitenden fördern und von der erfolgreichen Anwendung und Verknüpfung der Trainingsinhalte (erfolgreiche Kommunikation, interaktionale Emotionsregulation und stärkenorientierte Leistungsbeurteilung) zu komplexeren Führungskompetenzen im Arbeitsalltag abhängen. Dafür wurden Skalen zu transformationalem, transaktionalem und interaktionalem Führungsverhalten aus bereits validierten Fragebogeninstrumenten verwendet. Für Skalen des transformationalen und transaktionalen Führungsverhaltens wurde die Kurzform des Multifactor Leadership Questionnaire (MLQ 5x; Bass \& Avolio, 1995) bzw. dessen deutsche Übersetzung nach Felfe und Goihl (2002) sowie Felfe (2006) herangezogen. Items zur Messung interaktionalen Führungsverhaltens basierten auf der Leader-Member-Exchange-7-Item-Skala (LMX7; Graen \& Uhl-Bien, 1995) bzw. deren deutsche Übersetzung von Schyns und Collani (2002). Aufgrund der Spezifität der Trainingsreihe und des Unternehmenskontextes mussten jedoch auch 
diese teilweise modifiziert werden, um als passende Evaluationskriterien genutzt werden zu können: Drei Items des MLQ 5x wurden nach Betriebsratsvorgaben aufgrund unpassender Formulierungen gestrichen (z. B. „Ich mache andere stolz darauf, mit mir zusammenzuarbeiten“). Für transaktionale Führungsverhaltensweisen wurde nur die Subskala „Kontingente Belohnung“ genutzt, da „Management by Exception“ für das Führungsverständnis des Unternehmens nicht ganz passend war. Zudem wurden die Erfolgsskalen mit Ausnahme eines Items („Ich habe die Fähigkeit, berufliche Bedürfnisse und Interessen Anderer zu erkennen und zu berücksichtigen“) - was hier als Aspekt der transformationalen Führung verwendet wurde - nicht miterfasst, da diese Items als nicht passend zum Unternehmenskontext und den Trainingsinhalten eingeschätzt wurden (z. B. „ICh sporne den Willen anderer zum Erfolg an“). Der LMX7 wurde um vier Items ergänzt, um die Skala auf die Trainingsinhalte auszurichten (z. B. „Ich lobe meine Mitarbeiter, wenn sie ihre Aufgaben gut erledigen“).

Da Veränderungen auf diesen komplexeren Transferskalen erst nach einer ausreichenden Transferzeit sinnvoll erfasst werden können und aufgrund der begrenzten Itemzahl in den bausteinspezifischen Fragebögen, wurden die Skalen des „weiten Transfers“ nur in der übergeordneten Vor- und Nachbefragungen erfasst. Die internen Konsistenzen der beiden Messzeitpunkte lagen sehr nahe beieinander. Allerdings war die Skalengüte sehr durchmischt und zeigte $\alpha$-Werte zwischen .55 und .92. Während transformationales und interaktionales Führungsverhalten sehr gute Skalengüte aufwiesen, zeigte die Skala des transaktionalen Führungsverhaltens („Kontingente Belohnung“) nur suboptimale Skalengüte. Dafür kann jedoch auch die geringe Itemzahl mitverantwortlich sein. Dennoch sollten Analysen dieser Skala mit Vorsicht interpretiert werden.

\section{Resultate}

Diese Evaluationsebene soll gemäß der Transferdefinition nach Baldwin \& Ford (1988) prüfen, ob das Training zu bedeutsamen positiven Veränderungen in der Arbeits- bzw. Führungsleistung resultiert. Grundsätzlich könnte diese Ebene des Transfererfolgs auch durch verschiedene Unternehmenskennzahlen wie Fluktuation, Arbeitsunfähigkeitsfälleund -tage sowie Erfolgskennzahlen (Umsatz, Return on Investment) operationalisiert werden. Wie bereits erläutert, ist diese Evaluationsebene jedoch nicht eindeutig auf den Trainingserfolg zurück zu führen, da dabei eine Vielzahl von Faktoren zusammenwirken, wobei einige sogar außerhalb des Einflussbereichs einer Organisation liegen (z. B. Wettbewerbssituation oder Arbeitslosenquote). Im betrachteten Unternehmenskontext war auch vor der Trainingsplanung eine Expansion und Marktsegmentverschiebung geplant, was sich deutlich und relativ direkt auf Erfolgskennzahlen auswirkt, während die erfolgreiche Entwicklung von Führungskräften zeitverzögert und indirekt darauf einwirkt. Somit 
sind Erfolgskennzahlen hier keine sinnvollen Indikatoren. Zudem haben Datenschutzverantwortliche und Betriebsrat der Nutzung von Fluktuationsdaten und Ausfalltagen nicht zugestimmt. Auch wenn es unter dem Aspekt einer ganzheitlichen Betrachtung interessant wäre, auch Wirtschaftlichkeitskennzahlen im Prä-Post-Vergleich zu erfassen, erscheint es im Rahmen dieser Erhebungen nicht sinnvoll bzw. möglich diese zur Erklärung der Transferwirkungen bzw. dem Beleg des Trainingserfolgs heranzuziehen. Stattdessen wurden Bewertungen der Verbesserung der Führungskommunikation und des Führungsverhaltens als sinnvolle und praktikable Indikatoren des resultierenden Transfererfolgs erachtet. Beide Aspekte wurden durch direkte Veränderungsmessungen mit je einem Single-Item nach der festgelegten Transferzeit erfasst. Da der Erfolg der Anwendung von Führungskompetenzen maßgeblich von der Wahrnehmung betroffener Mitarbeitender abhängt, wurde die Selbsteinschätzung der Transferresultate durch Mitarbeitendeneinschätzungen ergänzt. Die verwendeten Items wurden folgendermaßen abgefragt und auf einer Skala von -2 („deutlich verschlechtert“) bis +2 („deutlich verbessert") bewertet:

- „Insgesamt hat sich die Kommunikation mit meinen Mitarbeitern/meiner Führungskraft seit der Trainingsreihe..."

(Selbsteinschätzung: $N=40, M=3.90 \mathrm{SD}=0.71$; Mitarbeitendeneinschätzung: $\mathrm{N}=35, \mathrm{M}=3.65, \mathrm{SD}=0.76$ )

- „Insgesamt hat sich mein Führungsverhalten/das Führungsverhalten meiner Führungskraft seit der Trainingsreihe..."

(Selbsteinschätzung: $N=40, M=3.75 \mathrm{SD}=0.63$; Mitarbeitendeneinschätzung: $\mathrm{N}=35, \mathrm{M}=3.67, \mathrm{SD}=0.65)$ 


\subsection{Kapitelzusammenfassung}

Zur Beantwortung der Forschungsfragen wurde eine partizipative und integrative (methoden-, theorie- und praxisbasierte) Selbstevaluation zur Ergebnis- und Prozessüberprüfung ohne Vergleichsintervention durchgeführt. Gegenstand des Forschungsprojektes war dabei nicht nur eine maßgeschneiderte Evaluation mit längsschnittlicher Datenerhebung zur Überprüfung von Transfererfolg und -mechanismen, sondern auch die wissenschaftlich fundierte Konzeption und Durchführung von Trainingseinheiten für nachhaltig wirksame Führungskräfteentwicklung. Auf dieser Basis wurde gemäß Empfehlungen der Trainingsforschung eine prozessbegleitende multidimensionale-multimethodale Evaluation zur Überprüfung der Trainingswirksamkeit sowie des Wirkmodells entwickelt und angewendet. Dafür war ein komplexes, ganzheitliches und langzeitlich angelegtes Studiendesign erforderlich. Das in diesem Kapitel beschriebene methodische Vorgehen wird hier anhand der drei grundlegenden methodischen Herausforderungen einer Evaluation nach Bungard, Holling und Schultz-Gambard (1996) zusammengefasst:

1. Verfahrensselektion durch die Wahl eines geeigneten Studiendesigns und statistischen Auswertungsverfahrens

2. Auswahl und Integration der Evaluationskriterien durch die Konzeption von Fragebögen und weiteren Evaluationskriterien (Operationalisierung)

3. Auswertung und Interpretation der Ergebnisse durch Hypothesenprüfung, Analysen und kritische Reflektion

Im Rahmen der Verfahrensselektion bezüglich Evaluationsdesign und -gegenstand wurde für die Beantwortung der Forschungsfragen eine längsschnittliche Feldstudie in einem repräsentativen bzw. prototypischen deutschen Unternehmen mittels EingruppenPlan mit Mehrfachmessung (vor, unmittelbar nach und ca. 7 Monate nach den Trainingseinheiten) als geeignet und umsetzbar für die Überprüfung des Transfererfolgs betrachtet. Um möglichst viele konfundierende Faktoren der Evaluationskriterien kontrollieren zu können und dennoch repräsentative Ergebnisse zu erhalten, wurde das Forschungsprojekt in Kooperation mit einem deutschen, international tätigen Unternehmen einer Schlüsselindustriebranche (Maschinenbau) durchgeführt, das ca. 450 Mitarbeitende in Verwaltung und Produktion beschäftigt. Die dadurch realisierte Stichprobengröße umfasste 66 Führungskräfte, von denen $42 \mathrm{im}$ Jahr 2014 und weitere 24 Führungskräfte im Jahr 2016 an der gleichen Trainingsreihe und Evaluation teilnahmen. Dadurch konnte ein kombiniertes Verfahren aus Eingruppen-Prä-Posttestplan und Wartelistenkontrollgruppenvergleich zur ergebnisorientierten Wirksamkeitsprüfung realisiert werden, um 
die Vor- und Nachteile der jeweiligen Designs zu kompensieren. Hierfür wurde eine Validierung der Ergebnisse aus dem Eingruppen-Prä-Posttestplan mit allen 66 Teilnehmenden durch einen zusätzlichen Vergleich zwischen den Teilnehmenden aus 2014 und 2016 geplant. So konnte überprüft werden, ob die Postwerte der indirekten Veränderungsmessungen bezüglich Reaktion, Kompetenzzuwachs und Transferverhalten von früher trainierten Führungskräften signifikante Verbesserungen im Vergleich zu den Präwerten der später trainierten Führungskräften zeigten. Da die Resultatebene auf direkten Veränderungsmessungen durch Postwerte basiert, fand deren Validierung durch zusätzliche Überprüfung an einer weiteren Datenquelle statt. Für die Bewertung des Transfererfolges von Führungskräftetrainings gelten Mitarbeitendeneinschätzungen als entscheidendes Kriterium, weshalb diese als zweite Quelle dienten. Basierend auf diesem Studiendesign wurden t-Tests für die ergebnisorientierten Analysen als statistische Auswertungsverfahren gewählt.

Für die prozessorientierten Analysen zur Erklärung individuellen Trainings- und Transfererfolges auf Mikroebene (Hypothese 2 und 3) wurden individuelle Unterschiede der Teilnehmenden, die mit der Intervention bzw. den abhängigen Variablen interagieren könnten, durch Einbezug von Ausgangswerten und demographischen Variablen kontrolliert. Da für diese Zusammenhangshypothesen die individuelle Wahrnehmung der Teilnehmenden entscheidend und valide ist (Chiaburu et al., 2010; Facteau et al., 1995; Podsakoff et al., 2012; Spector, 2006), wurde hierfür keine weitere Datenquelle oder Vergleichsgruppe herangezogen. Aufgrund der Stichprobengröße eignen sich für diese Forschungsfragen (hierarchische) Regressionsanalysen mit Kontrolle von Ausgangswerten und relevanten demographischen Variablen als statistisches Auswertungsverfahren (Dalecki \& Willits, 1991). Daher wurde diese Vorgehensweise gewählt.

Die Operationalisierung durch Festlegung der Evaluationskriterien orientierte sich an etablierten Modellen zum Trainings- und Transfererfolg. Die Trainingswirksamkeit wurde multidimensional basierend auf Kirkpatricks Evaluationsmodell $(1967,1994)$ durch Reaktion, Kompetenzentwicklung, Transferverhalten und -resultate operationalisiert. Die Erfassung der Transferfaktoren erfolgte ebenfalls multidimensional und basierte auf einer Integration der Transferforschung und -modelle von Noe und Schmitt (1986), Baldwin und Ford (1988) sowie Holton et al. (2000) - siehe auch Abbildung 12 auf Seite 87. Entsprechend wurden die empirisch bestätigten Transferfaktoren der drei übergeordneten Dimensionen Teilnehmendenmerkmale, Trainingsgestaltung und Arbeitsumgebung operationalisiert. Zur Erfassung der Evaluationskriterien wurden Fragebögen eingesetzt, die basierend auf Trainingsinhalt und -kontext maßgeschneidert konstruiert wurden. Auch wenn dieses Vorgehen wissenschaftlichen Standards und Empfehlungen zur Mi- 
nimierung von Messfehlern entgegensteht, war aufgrund erhebungsökonomischer Anforderungen und spezifischer Trainingsinhalte (wie in Evaluationsstudien im Feld meist anzutreffen) auch hier der Einsatz selbstkonstruierter Skalen erforderlich. Ein Validierungsprozess war dabei weder zeitlich noch finanziell möglich. Um das daraus resultierende Risiko von Unreliabilität und geringer Validität zu minimieren, orientierten sich Aufbau und Itemformulierungen an validierten Skalen und Fragebogeninstrumenten. Nach Skalenoptimierungen zeigten fast alle Skalen über alle Messzeitpunkte hinweg akzeptable bis gute interne Konsistenz.

Die Auswertung und Interpretation der quantitativen Fragebogendaten erfolgte in drei übergeordneten Schritten, anhand von Verfahren, die für die Überprüfungen der Hypothesen mit dem gewählten Studiendesign den bestmöglichen Erkenntnisgewinn liefern:

1. Datenauswertung zur Hypothesenprüfung

2. Explorative Auswertungen zur weiteren Ergründung der Transfermechanismen

3. Kritische Reflexion des Vorgehens und der Ergebnisse durch Diskussion und Ableitung eines fundierten Leitfadens für systematisches Transfermanagement

Diese Schritte sind in den beiden nachfolgenden Kapiteln beschrieben. 


\section{Datenanalysen}

In diesem Kapitel wird die Überprüfung der von bestehenden Theorien und Forschungserkenntnissen abgeleiteten Hypothesen (siehe Kapitel 4 ab Seite 85) anhand der vorliegenden Daten beschrieben und kritisch reflektiert. Im Rahmen der prozessorientierten Wirksamkeitsüberprüfung werden ergänzend explorative Analysen zur tiefergehenden Ergründung der Transfermechanismen dargestellt und diskutiert. Um diese Analysen einordnen zu können, werden einleitend noch deren generelle Vorgehensweisen beschrieben.

\subsection{Generelle Vorgehensweisen}

Für die Datenanalysen wurde die Statistiksoftware SPSS Statistics Version 25.0 von IBM verwendet. Für die Hypothesentestungen wurde die gängige Konvention einer $5 \%$ igen Irrtumswahrscheinlichkeit als Entscheidungskriterium für die finale Annahme bzw. Ablehnung einer Hypothese herangezogen. Wobei auch noch bei einem marginalen Signifikanzniveau bis $10 \%$ gegebenenfalls entsprechende Schlussfolgerungen interpretiert wurden. Da sich alle Hypothesentestungen gezielt und umfassend auf ein Transfermodell bezogen, können die verschiedenen Hypothesentestungen am gleichen Datensatz nicht als unabhängig betrachtet werden. Zum Zwecke der Konstanthaltung möglichst vieler konfundierender Variablen war es jedoch in diesem Kontext erforderlich auf nur eine Stichprobe zurück zu greifen. Das bedingte allerdings eine Inflation des Alphafehlers. Diesem Problem kann durch Alpha-Adjustierungen begegnet werden. Allerdings unterliegen die gängigen Korrekturen des Alpha-Niveaus (z. B. Bonferroni- oder Bonferroni-Holm-Adjustierung) zunehmender Kritik bezüglich Verlust der statistischen Power (Field, 2018). Daher kann die Sinnhaftigkeit einer suboptimalen Korrektur auf Basis der festgelegten Konvention in Frage gestellt werden. Zudem ist ein Signifikanzniveau von $5 \%$ bei einer kleinen Stichprobe ohnehin als eher konservativ zu betrachten, sodass eine Adjustierung die Teststärke noch weiter mindern würde. Daher wurde es hier als methodisch vertretbar erachtet, zugunsten der Übersichtlichkeit und des Erkenntnisgewinns auf eine Alpha-Adjustierung zu verzichten. Stattdessen wurde der Alphafehlerinflation durch a priori aufgestellten Hypothesen bzw. gezielten theoriegeleiteten Testungen begegnet. Zudem wurden die Hypothesen soweit anhand bisheriger Forschung als sinnvoll erachtet an verschiedenen abhängigen Variablen überprüft, um zu zeigen inwiefern unterschiedliche Operationalisierungen ein konsistentes Ergebnisbild ergeben. Wobei angenommen werden kann, dass bei relativ konsistenten Ergebnissen zufallsbedingte Signifikanzen bzw. Alphafehler unwahrscheinlich sind. 
Eine weitere generelle Vorgehensweise bei den nachfolgenden Hypothesentestungen bezieht sich auf die Bewertung der erforderlichen Stichprobengröße für Regressionsanalysen. Welche Stichprobengröße für reliable Testmodelle der regressionsanalytischen Hypothesentestungen benötigt werden, haben Miles und Shevlin (2001) aus den bisherigen (teilweise kontroversen) Empfehlungen anschaulich zusammengefasst und dabei spezifische Analysebedingungen berücksichtigt. Auch wenn generell empfohlene Daumenregeln von zehn bis fünfzehn Fälle pro Prädiktor existieren (Field, 2018), ist deren unhinterfragte Anwendung kritisch zu betrachten. Denn die benötigte Stichprobengröße ist nicht nur von der Anzahl der Prädiktoren abhängig, sondern vor allem auch von deren erwarteten Vorhersagekraft bzw. der Vollständigkeit der Testmodelle. So können Analysen auch mit kleineren Stichprobengrößen reliabel sein, wenn sie theoriegeleitet bestätigte Prädiktoren möglichst umfassend berücksichtigen und große Effekte zu erwarten sind. Um daher für die vorliegenden Fragestellungen eine akkuratere Vorgabe der benötigten Stichprobengröße zu erhalten, werden die Diagramme basierend auf Miles und Shevlin (2001) herangezogen. Abbildung 20 enthält ein darauf basierendes Diagramm zur Ermittlung benötigter Stichprobengrößen abhängig von Anzahl und Effektstärke der Prädiktoren um eine hohe Teststärke von .8 gemäß dem Cohens Benchmark (1988) zu erreichen.

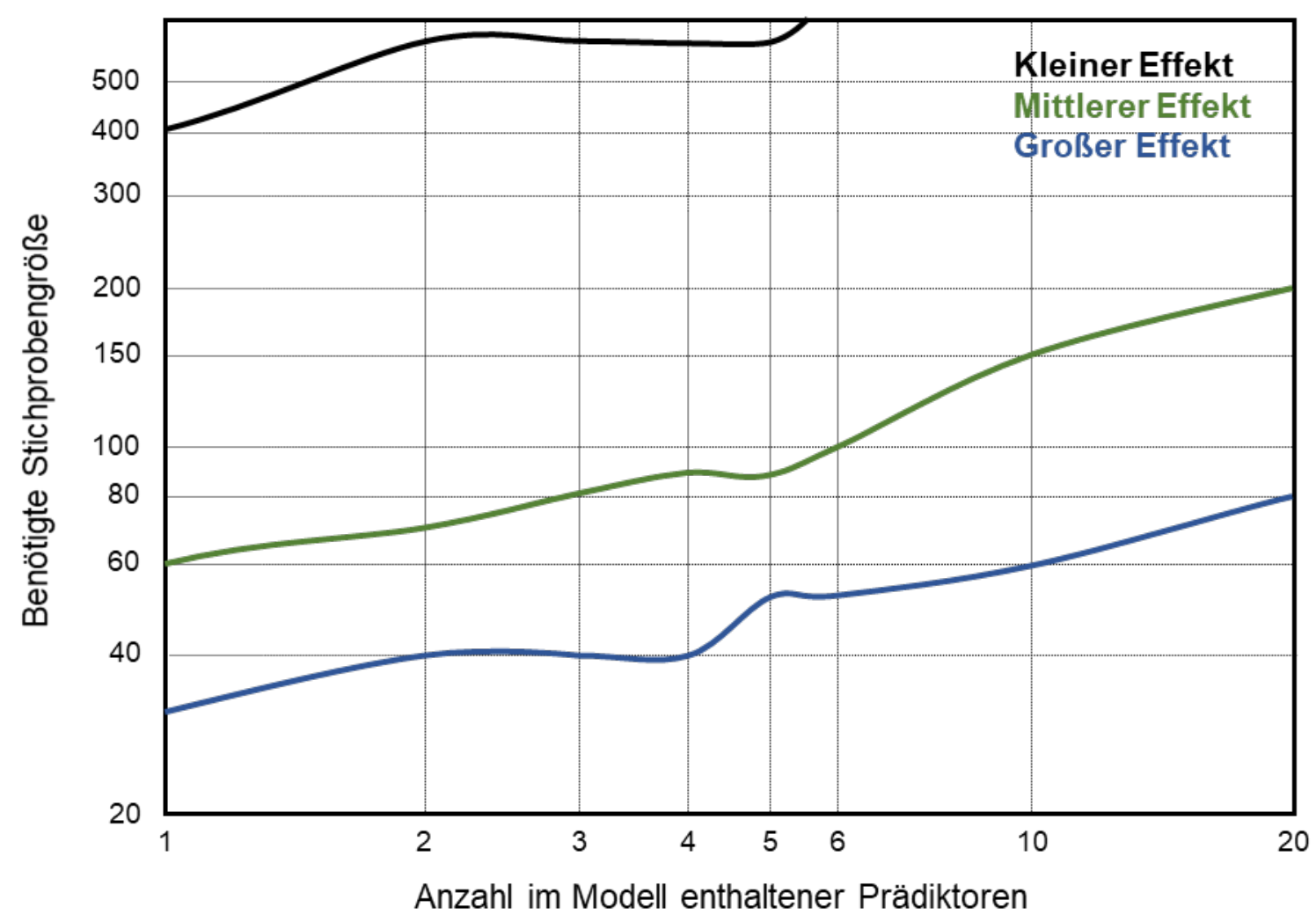

Abbildung 20:Benötigte Stichprobengrößen für Auswertungen mit multiplen Regressionsanalysen mit einer Teststärke von .8 nach Miles und Shevlin (2001); eigene Darstellung basierend auf Field (2018), S. 390. 
Da für die Hypothesentestungen nur Prädiktoren aus etablierten Transfermodellen verwendet wurden, wurde für die nachfolgenden Testungen die unterste Linie von Abbildung 20 als Orientierung herangezogen. Demnach können bei der vorliegenden Stichprobengröße bis zu vier Prädiktoren in einem Modell getestet werden, wenn eine Teststärke von .8 erreicht werden soll. Werden mehr Prädiktoren in die Analysen aufgenommen, sinkt die Wahrscheinlichkeit, dass ein existierender Effekt inferenzstatistisch gefunden wird bzw. dass die Regressionsmodelle ausreichend reliabel sind. Daher lagen nachfolgende Analysen möglichst nahe an dieser Maximalzahl von vier Prädiktoren. Dennoch werden zugunsten möglichst vollständiger Modelle gegebenenfalls bis zu zehn Prädiktoren als noch vertretbar angenommen. Denn trotz geringerer Teststärke kann der Erkenntnisgewinn dadurch höher sein, als bei Nutzung unvollständiger Modelle (Field, 2018).

Zur Veränderungsmessung von Trainingsoutcomes und Transfervariablen wurden, wie von Dalecki und Willits (1991) empfohlen, die Postwerte als abhängige Variablen und deren Ausgangswerte als Kontrollvariable verwendet. Dafür wurden hierarchische Regressionsanalysen durchgeführt bei denen, neben den als relevant identifizierten demographischen Variablen, auch die Ausganswerte ins erste Modell aufgenommen wurden. Im zweiten Schritt wurden die in den Hypothesen angenommenen Prädiktoren ins Regressionsmodell aufgenommen. Zur Identifikation relevanter demographischer Variablen wurde ihre Korrelationen nach Pearson mit den betrachteten abhängigen Variablen auf Signifikanz geprüft und nur bei Signifikanz ( $p<0.05,2$-seitig) in das Testmodell zur Hypothesenprüfung aufgenommen. Dieses Vorgehen wurde gewählt, da durch Kontrollvariablen, die keinen Einfluss auf die abhängigen Variablen haben, die Ergebnisse verzerrt werden könnten. Zudem konnten dadurch in den finalen Testmodellen möglichst wenige aber dennoch alle relevanten Prädiktoren berücksichtigt werden, was sich positiv auf die statistische Power auswirkt.

Die Voraussetzungsprüfung der Regressionsanalysen orientierte sich an Field (2018) und umfasste folgende Kriterien:

- Erforderliche Variablentypen sind vorhanden:

Alle Kontrollvariablen und Prädiktoren konnten basierend auf einer fünfstufigen Likert-Skala als metrisch betrachtet werden oder sind dichotom kategorial; alle abhängigen Variablen konnten basierend auf einer fünfstufigen Likert-Skala als metrisch betrachtet werden.

- Null-Varianzen aller Variablen konnten anhand von deskriptiven Analysen ausgeschlossen werden. 
- Einer Unkorreliertheit der Prädiktoren mit „Externen Variablen“, die in den Modellen nicht berücksichtigt wurden, sollte durch die Verwendung theoriebasierter kompletter Modelle und Vorabprüfung relevanter demographischer Variablen auf die betrachteten abhängigen Variablen (siehe oben) entgegengewirkt werden.

- Multikolinearität der Modellvariablen wurde durch Prüfung der Inter-ItemKorrelationen mit einem Grenzwert von unter .9 sowie durch Prüfung, ob alle Varianzinflationsfaktoren (VIF) kleiner 10 sind und deren Mittelwerte nahe 1 oder sogar darunter liegen, ausgeschlossen.

- Als Prüfungskennwert für die Unabhängigkeit der Residuen wurde der Durbin-Watson-Wert verwendet, der nahe 2 bzw. zwischen 1 und 3 liegen sollte.

- Für die Prüfung auf Homoskedastizität, Linearität und Normalverteilung der Residuen wurden fallweise Diagnosen bezüglich Ausreißer der Standardresiduen mit einem Betrag von größer 2 (wenn davon mehr als 5\% der Fälle liegen, kann es als Indikator für unzureichende Modellpassung gewertet werden) sowie grafische Prüfungen der Standardresiduen durch Histogramme, Streudiagramme und P-P-Plots (der Zusammenhang zwischen erwarteten und beobachteten Werten sollte nahe 1 liegen) durchgeführt.

- Eine Kreuzvalidierung der Regressionsmodelle wurde zusätzlich zu den Standardprüfungen anhand Modellzusammenfassungen und ANOVAs durch einen Abgleich von Wherry's korrigiertem $\mathrm{R}^{2}$ mit dem $\mathrm{R}^{2}$ der Modelle vorgenommen.

Wenn bei den jeweiligen Analysen keine weiteren Anmerkungen dazu gemacht werden, können die Voraussetzungen der Regressionsanalysen auf dieser Basis als erfüllt gelten. Die Analysen wurden zudem anhand fallweiser Diagnostik auf Ausreißer überprüft. Wenn nicht anders angegeben wurden keine Ausreißer identifiziert, die die Ergebnisse bedeutsam beeinflussen. Weitere spezifische Annahmen und Vorgehensweisen bezüglich der Überprüfung einzelner Hypothesen sind in den nachfolgenden Abschnitten bei den einzelnen Analysen erläutert. 


\subsection{Ergebnisorientierte Wirksamkeitsüberprüfung}

Im Rahmen der ergebnisorientierten Evaluation wurde Hypothese 1 „Ein transferförderliche gestaltetes Training ist wirksam (a) bezüglich kurzfristigem Trainingserfolg durch positive Trainingsreaktion (Nützlichkeitseinschätzung und Transfermotivation) und Kompetenzentwicklung sowie (b) bezüglich langfristigem Transfererfolg durch Transferverhalten (naher und weiter Transfer) und dessen Resultate (Verbesserung in Führungskommunikation und -verhalten)“ anhand verschiedener t-Tests überprüft. Für die Kriterien, die nur retrospektiv erfasst werden konnten (Nützlichkeitseinschätzungen der Reaktionsebene und Resultatebene), wurden einseitige Eingruppen-t-Tests mit einem Testwert von 3 (Mittelwert der Likert-Skala) durchgeführt. Damit wurde überprüft, ob der Nutzen des Trainings bzw. die Veränderung des Führungsverhaltens signifikant positiv eingeschätzt wurde. Für die indirekten Veränderungsmessungen wurden einseitige tTests verbundener Stichproben durchgeführt, um zu überprüfen, ob die Postwerte nach dem Training eine signifikante Verbesserung der Ausgangswerte zeigten. Die Ergebnisse sind in Tabelle 8 zusammengefasst.

Tabelle 8: Eingruppen-(Prä-)Postvergleich für Trainings- und Transfererfolgskriterien

\begin{tabular}{|c|c|c|c|c|c|c|c|c|c|c|c|c|c|}
\hline \multirow{2}{*}{\multicolumn{2}{|c|}{ Trainings- und Transfererfolg }} & \multirow{2}{*}{$\mathrm{N}$} & \multirow{2}{*}{$\begin{array}{l}\text { Test- } \\
\text { wert }\end{array}$} & \multirow{2}{*}{ Prä } & \multicolumn{2}{|c|}{ Mittelwert } & \multicolumn{3}{|c|}{$\begin{array}{l}\text { Standard- } \\
\text { abweichung }\end{array}$} & \multirow[t]{2}{*}{$\mathrm{T}$} & \multirow{2}{*}{ df } & \multirow{2}{*}{$\begin{array}{l}\text { Sig. } \\
\text { (1-seitig) }\end{array}$} & \multirow{2}{*}{$\begin{array}{c}\text { Effektstärke } \\
\text { (r) }\end{array}$} \\
\hline & & & & & Post & Differenz & Prä & Post & Differenz & & & & \\
\hline \multirow[t]{9}{*}{ Reaktion } & Inhaltsrelevanz TB1 & 60 & 3 & & 3.45 & 0.45 & & 0.49 & & 7.15 & 59 & 0.000 & 0.68 \\
\hline & Inhaltsrelevanz TB2 & 55 & 3 & & 4.07 & 1.07 & & 0.40 & & 19.71 & 54 & 0.000 & 0.94 \\
\hline & Inhaltsrelevanz TB3 & 45 & 3 & & 4.20 & 1.20 & & 0.51 & & 15.87 & 44 & 0.000 & 0.92 \\
\hline & Transferdesign TB1 & 60 & 3 & & 3.80 & 0.80 & & 0.49 & & 12.69 & 59 & 0.000 & 0.86 \\
\hline & Transferdesign TB2 & 55 & 3 & & 4.24 & 1.24 & & 0.37 & & 24.59 & 54 & 0.000 & 0.96 \\
\hline & Transferdesign TB3 & 45 & 3 & & 4.36 & 1.36 & & 0.40 & & 23.03 & 44 & 0.000 & 0.96 \\
\hline & Transfermotivation TB1 & 34 & & 3.77 & 3.83 & 0.06 & 0.64 & 0.45 & 0.69 & 0.54 & 33 & 0.298 & 0.09 \\
\hline & Transfermotivation TB2 & 41 & & 3.80 & 3.98 & 0.18 & 0.63 & 0.54 & 0.50 & 2.29 & 40 & 0.014 & 0.34 \\
\hline & Transfermotivation TB3 & 28 & & 3.98 & 4.14 & 0.16 & 0.60 & 0.53 & 0.46 & 1.86 & 27 & 0.037 & 0.34 \\
\hline \multirow[t]{3}{*}{ Lernen } & Kompetenzentwicklung TB1 & 36 & & 3.86 & 4.20 & 0.34 & 0.37 & 0.31 & 0.33 & 6.27 & 35 & 0.000 & 0.73 \\
\hline & Kompetenzentwicklung TB2 & 41 & & 3.74 & 3.94 & 0.19 & 0.38 & 0.42 & 0.37 & 3.37 & 40 & 0.001 & 0.47 \\
\hline & Kompetenzentwicklung TB3 & 28 & & 4.09 & 4.37 & 0.29 & 0.35 & 0.37 & 0.36 & 4.19 & 27 & 0.000 & 0.63 \\
\hline \multirow[t]{6}{*}{ Verhalten } & Naher Transfer TB1 & 28 & & 3.43 & 3.63 & 0.19 & 0.43 & 0.41 & 0.52 & 1.95 & 27 & 0.031 & 0.35 \\
\hline & Naher Transfer TB2 & 28 & & 3.33 & 3.65 & 0.32 & 0.49 & 0.56 & 0.50 & 3.40 & 27 & 0.001 & 0.55 \\
\hline & Naher Transfer TB3 & 26 & & 4.03 & 4.18 & 0.15 & 0.44 & 0.42 & 0.43 & 1.81 & 25 & 0.042 & 0.34 \\
\hline & Transformationale Führung & 40 & & 3.66 & 3.80 & 0.14 & 0.45 & 0.42 & 0.38 & 2.28 & 39 & 0.014 & 0.34 \\
\hline & Transaktionale Führung & 40 & & 3.63 & 3.82 & 0.19 & 0.69 & 0.55 & 0.66 & 1.79 & 39 & 0.041 & 0.28 \\
\hline & Interaktionale Führung & 39 & & 4.00 & 4.06 & 0.05 & 0.42 & 0.34 & 0.40 & 0.80 & 38 & 0.213 & 0.13 \\
\hline \multirow[t]{4}{*}{ Resultate } & Verbesserte Führungskommunikation & 40 & 3 & & 3.90 & 0.90 & & 0.71 & & 8.03 & 39 & 0.000 & 0.79 \\
\hline & Verbessertes Führungsverhalten & 40 & 3 & & 3.75 & 0.75 & & 0.63 & & 7.52 & 39 & 0.000 & 0.77 \\
\hline & $\begin{array}{l}\text { Verbesserte Führungskommunikation } \\
\text { (Mitarbeitendeneinschätzung) }\end{array}$ & 35 & 3 & & 3.65 & 0.65 & & 0.76 & & 5.03 & 34 & 0.000 & 0.65 \\
\hline & $\begin{array}{l}\text { Verbessertes Führungsverhalten } \\
\text { (Mitarbeitendeneinschätzung) }\end{array}$ & 35 & 3 & & 3.67 & 0.67 & & 0.65 & & 6.10 & 34 & 0.000 & 0.72 \\
\hline
\end{tabular}

Anmerkungen. $\mathrm{N}=$ Anzahl von Teilnehmenden, die die analysierten Skalen und Messzeitpunkte beantwortet haben.

Der Eingruppen-Prä-Postvergleich zeigte für fast alle Trainings- und Transfererfolgskriterien signifikante bis hochsignifikante Effekte und damit ein relativ konsistentes Ergebnis. Sowohl die Teilnehmenden als auch ihre Mitarbeitenden nehmen nach ca. sieben 
Monate nach der Trainingsreihe auch auf der Resultatebene eine Verbesserung in der Führungskommunikation und sogar auch im allgemeinen Führungsverhalten war. Allerdings ist anzumerken, dass die Mittelwerte alle unter 4 lagen, weshalb hier nur von einer leichten Verbesserung und nicht von einer deutlichen Verbesserung gesprochen werden kann. Konsistent zur bisherigen Trainingsforschung (Taylor et al., 2009) zeigten dabei die Mitarbeitendeneinschätzungen geringere Veränderungen als die Selbsteinschätzungen.

Nur der kurzfristige Trainingserfolg des Trainingsbausteins 1 bezüglich Förderung der Transfermotivation sowie der langfristige Transfererfolg bezüglich interaktionalem Führungsverhalten konnte auf der Makroebene nicht bestätigt werden. Dagegen zeigte auch transaktionales Führungsverhalten, trotzt suboptimaler interner Konsistenz, eine signifikante und bedeutsame Verbesserung. Eine mögliche Erklärung für den ausgebliebenen kurzfristigen Trainingserfolgs des Trainingsbausteins 1 bezüglich Förderung der Transfermotivation, könnte darin liegen, dass Trainingsbaustein 1 als Einführungsveranstaltung inhaltlich und methodisch auf Wissensvermittlung und Vorbereitung der weiteren Trainingseinheiten ausgerichtet war. Dafür sprechen auch die relativ geringen Nützlichkeitseinschätzungen (insbesondere bezüglich Inhaltsrelevanz) im Vergleich mit den anderen Trainingsbausteinen. Ein weiterer Grund könnte auch darin liegen, dass nach einer eintägigen Maßnahme noch nicht ausreichend Möglichkeiten bestanden, entwickelte Kompetenzen und ihre Umsetzung zu reflektieren (siehe auch Massenberg \& Kauffeld, 2015). Zudem ist Transfermotivation ein eher distaler Indikator der Reaktionsebene, worauf auch die geringeren Effektstärken im Vergleich zu den sehr hohen Werten der Nützlichkeitseinschätzungen hinweisen.

Der nicht-bestätigte langfristige Transfererfolg bezüglich interaktionalem Führungsverhalten, könnte einerseits darin begründet liegen, dass bereits der Ausgangswert mit 4.00 auf einer fünfstufigen Skala relativ hoch war (Deckeneffekt). Andererseits könnte ein Grund aber auch in der relativ großen Distanz zwischen Training und Transferkriterium liegen, da transformationale und transaktionale Verhaltensweisen näher an Trainingsinhalt und nahem Transfer lagen. Zudem hängt interaktionale Führung noch stärker von der Beziehung mit bzw. Wahrnehmung von den unterstellten Mitarbeitenden ab, als transformationale und interaktionale Führung (Graen \& Uhl-Bien, 1995; Schyns, 2002). Daher kann hier eine längere Transferzeit erforderlich sein, um signifikante Effekte zu erhalten.

Für Hinweise darauf, inwiefern die signifikanten Ergebnisse bedeutsam sind, wurden zudem die Effektstärken der Tests überprüft. Bei Werten von .10 kann ein kleiner Effekt (1\% Varianzaufklärung), bei .30 ein mittlerer Effekt (9\% Varianzaufklärung) und ab .50 ein großer Effekt (25\% Varianzaufklärung) angenommen werden (Cohen, 1988, 1992). 
Alle signifikanten Ergebnisse zeigten demnach mittlere bis große Effekte und können daher als bedeutsamen Trainings- und Transfererfolg gelten. Während Nützlichkeitseinschätzungen der Reaktionsebene, Lernebene und Resultatebene große Effekte aufwiesen, lagen die Effektstärken des Transferverhaltens vorwiegend im mittleren Bereich. Beim Vergleich zwischen nahen und weiten Transferkriterien zeigten sich vergleichbare Effektstärken bei den signifikanten Ergebnissen. Einzige Ausnahme war der nahe Transfer des Trainingsbausteins 2. Dessen größeren Effekte im Vergleich zu Trainingsbaustein 1 könnte darin begründet liegen, dass Trainingsbaustein 1 als Einführungsveranstaltung inhaltlich und methodisch vorwiegend auf Wissensvermittlung und Vorbereitung der weiteren Trainingseinheiten ausgerichtet war, während insbesondere Trainingsbaustein 2 Methoden auf der Verhaltensebene fokussierte. Die größeren Effekte im Vergleich zu Trainingsbaustein 3 könnten dagegen in der fehlenden Auffrischungssitzung begründet liegen (die es nur für die ersten beiden Bausteine zu Beginn des jeweils darauffolgenden Bausteins gab, siehe Abschnitt 5.2.2 ab Seite 106). Andererseits könnte auch die suboptimale interne Konsistenz des nahen Transfers bei Trainingsbaustein 3 ein Grund für die geringere Effektstärke sein.

Aufgrund der Nachteile eines Eingruppen-Prä-Posttestplänen (siehe Abschnitt 5.2.1 ab Seite 92) wurde zusätzlich ein Wartelistenkontrollgruppenvergleich für Trainings- und Transfererfolgskriterien durchgeführt, für die Prä- und Postwerte erfasst wurden (siehe Tabelle 9). Dabei wurden die Postwerte der Führungskräfte, die bereits im Jahr 2014 trainiert wurden, mit den Präwerten der Führungskräfte, die im Jahr 2016 an der gleichen Trainingsreihe teilnahmen, verglichen. Dadurch sollten die gefundenen Effekte des Eingruppen-Prä-Posttestplans ergänzend mit einem alternativen statistischen Verfahren überprüft werden, um dessen Nachteile zu kompensieren bzw. dessen Ergebnisse zu validieren. Allerdings sind damit andere Nachteile verbunden: Einerseits muss beachtet werden, dass sich die Vergleichsgruppen bezüglich der Ausprägung von Hierarchiestufe und Alter hoch signifikant und bezüglich Führungserfahrung marginal signifikant unterschieden. Daher sollten die Ergebnisse des Wartelistenkontrollgruppenvergleichs unter Betracht der Möglichkeit interpretiert werden, dass sie durch diese systematischen Unterschiede der Kontrollvariablen beeinflusst wurden. Andererseits kann angenommen werden, dass der Trainings- und Transfererfolg der früher Trainierten Spillovereffekte durch Modelllernen im Arbeitsalltag der später trainierten Führungskräfte hatte. Dennoch waren alle Kriterienausprägungen der bereits Trainierten größer als der noch Untrainierten, wobei die meisten Erfolgskriterien der Experimentalgruppe auch (marginal) signifikante Überlegenheit im Vergleich zur Wartelistenkontrollgruppe aufwiesen. Mit Ausnahme des Transfereffekts bezüglich nahen Transfers von TB3 und weiten Transfers von transaktionalem Führungsverhalten, konnten dadurch der gefundene Trainings- und 
Transfererfolg des Eingruppen-Prä-Posttestplans bestätigt werden. Da diese beiden Skalen eine suboptimale interne Konsistenz zeigten, könnte dies zusammen mit den Nachteilen eines Kontrollgruppenplans bezüglich Kontrolle der Teilnehmendenmerkmale (Frese et al., 2003; Hunter \& Schmidt, 1990) - weshalb Within-Designs als belastbarer für Trainingsevaluationen gelten - der Grund für die nicht signifikanten Effekte sein.

Tabelle 9: Wartelistenkontrollgruppenvergleich für Trainings- und Transfererfolgskriterien

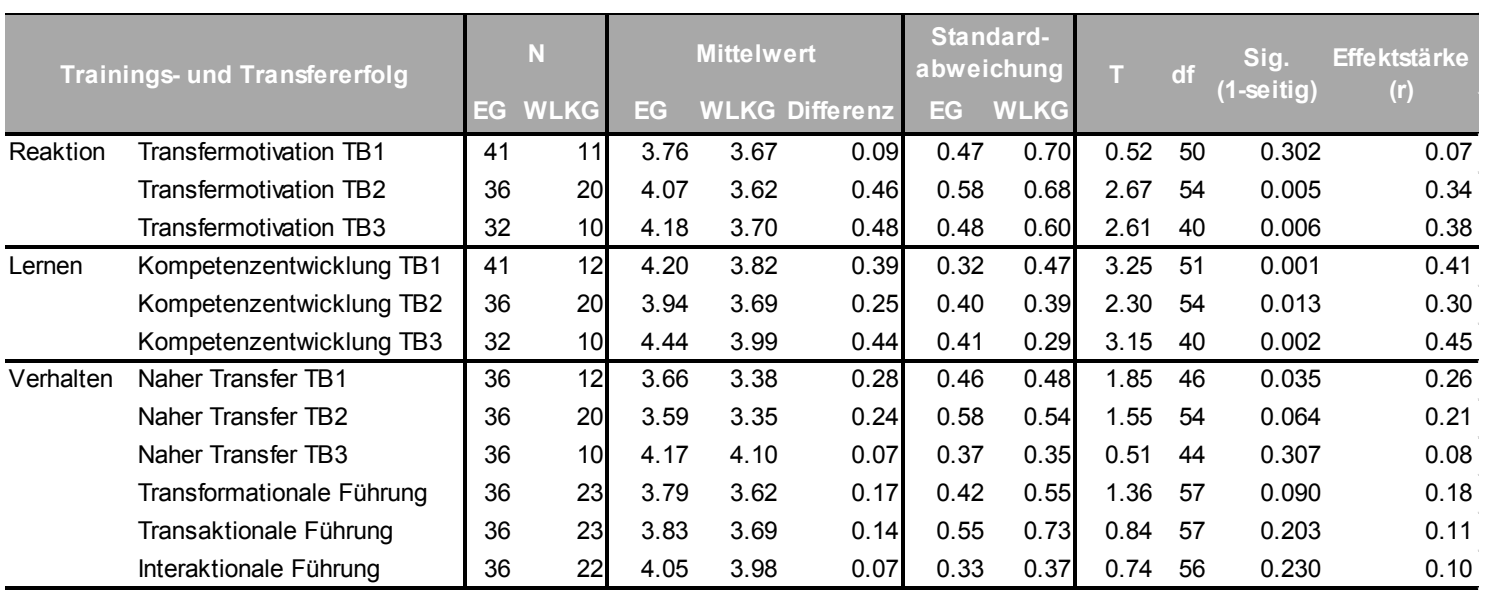

Anmerkungen. $\mathrm{N}=$ Anzahl von Teilnehmenden, die die analysierten Skalen und Messzeitpunkte beantwortet haben. EG = Experimentalgruppe. WLKG = Wartelistenkontrollgruppe.

Aufgrund des relativ konsistenten Ergebnisbildes der ergebnisorientierten Wirksamkeitsüberprüfung mit kombinierter Eingruppen-Wartelistenkontrollgruppen-Prä-Post-Überprüfung multidimensionaler Kriterien, kann damit Hypothese 1 als weitestgehend bestätigt gelten. Insgesamt weisen die Daten damit auf signifikante und bedeutsame Effekte bezüglich eines Trainings- und Transfererfolges auf gesamtorganisationaler Makroebene hin, der sich nicht nur unmittelbar nach dem Training in Reaktion und Kompetenzzuwachs zeigt, sondern auch noch ca. sieben Monate nach der Trainingsreihe im Transferverhalten und dessen Resultaten. Diese Ergebnisse der Selbsteinschätzung werden zudem anhand einer weiteren Datenquelle durch Mitarbeitendeneinschätzungen gestützt (wenn auch in etwas geringerem Maße im Vergleich zu Selbsteinschätzungen).

Damit bestätigt die vorliegende ergebnisorientierte Wirksamkeitsprüfung Ergebnisse bisheriger Forschung über die Wirksamkeit von Führungskräfteentwicklungsmaßnahmen, die in der aktuellsten Metaanalyse von Lacerenza et al. (2017) zusammengefasst wurde. Diese zeigte, dass entgegen der anhaltenden Kritik insbesondere bezüglich des Wirkungsgrades formaler „off-the-job“ Führungskräftetrainings zur Vermittlung von Softskills (Kaiser \& Curphy, 2013; Laker \& Powell, 2011; Poell, 2017; J. Schwartz et al., 2014; Staudt \& Kriegesmann, 1999), evaluierte Führungskräfteentwicklungsmaßnahmen substanzielle Effektstärken für die Reaktions- $(\delta=.63)$, Lern- $(\delta=.73)$, Transfer- $(\delta=.82)$ 
und Resultatebene $(\delta=.72$ ) hervorbringen können (siehe auch Abschnitt 3.1 ab Seite 31). Für zusätzlichen Erkenntnisgewinn wurden jedoch die zentralen Forschungslücken ergebnisorientierter Trainingsforschung adressiert. Einerseits wurde Fokus auf eine aussagekräftige Dokumentation relevanter Eigenschaften von Intervention (siehe Abschnitt 5.2 ab Seite 92) und Evaluation (siehe Abschnitt 5.1 ab Seite 91 sowie Abschnitt 5.3 ab Seite 109) gelegt, durch die eine präzise Einordnung der Ergebnisse sowie konkretere Handlungsimplikationen ableitbar sind. Andererseits wurde methodische Kritik bisheriger Forschung aufgegriffen, indem Trainings- und Transfererfolg im Rahmen einer kontrollierten Studie auf allen vier Dimensionen nach Kirkpatrick $(1967,1998)$ erfasst wurden, wobei auf präzise und erweiterte Kriterienoperationalisierung (Ford et al., 2018) sowie auf sinnvolle zeitliche Abstände bei der Erhebung des langfristigen Transfererfolgs (Arthur et al., 2003) geachtet wurde. Zudem wurde das Studiendesign im Rahmen der Möglichkeiten des Feldes optimiert (Martin et al., 2017). So wurde das in ergebnisorientierter Trainingsforschung gängige Studiendesign mit Eingruppen-Prä-Posttestplänen basierend auf Selbsteinschätzung, um einen Wartelistenkontrollgruppenvergleich und einer zusätzlichen Datenquelle auf der Resultatebene ergänzt. Die umfassendste Ergänzung bisheriger Forschung besteht schließlich in einer Integration von ergebnis- und prozessorientierter Wirksamkeitsprüfung, um die offene Frage zu ergründen, durch welche Faktoren und Maßnahmen Transfer gefördert werden kann. Der nachfolgende Abschnitt schließt dafür an die hier beschriebenen Ergebnisse auf organisationaler Makroebene an und beschreibt die Analyse der Wirkmechanismen im Trainings- und Transferprozess auf individueller Mikroebene. 


\subsection{Prozessorientierte Wirksamkeitsüberprüfung}

Zur Erklärung individueller Transferleistung auf Mikroebene wurde im Rahmen der prozessorientierten Evaluation zunächst der Wirkmechanismus anhand direkter bzw. primärer Transferdeterminanten gemäß Hypothese 2 überprüft. Explorativ wurde zudem die Vorhersagekraft aller erfassten Transferdeterminanten analysiert. In einem zweiten Schritt wurde gemäß Hypothese 3 überprüft, inwiefern die primären Transferdeterminanten durch die übergeordneten Skalen Teilnehmendenmerkmale, Trainingsgestaltung und Arbeitsumgebung vorhergesagt werden können. Auch hierzu wurde zudem explorativ die Vorhersagekraft aller erfassten Subskalen der Transferdeterminanten analysiert. Zugunsten möglichst umfassender Analysen der Transfermechanismen und einer Minimierung von Redundanzen wurde dafür eine Fokussierung auf den zentralen Trainingsbaustein 2 gewählt. Die Ergebnisse der Analysen zur Beantwortung der Forschungsfragen sind in nachfolgenden Abschnitt zusammengefasst. Weitere Analysen können bei der Autorin erfragt werden.

\subsubsection{Erklärung individueller Transferleistung}

\section{Hypothesenprüfung}

Im Rahmen der prozessorientierten Evaluation wurde Hypothese 2 „Transfer kann durch (a) Kompetenz, (b) Transfermotivation und (c) -möglichkeit maßgeblich vorhergesagt werden, (d) wobei Kompetenz den größten Einfluss zeigt" anhand von hierarchischen Regressionsanalysen überprüft. Dabei wurde für Ausgangswerte der Transferkriterien sowie gegebenenfalls für relevante demographische Variablen kontrolliert. Zur Identifikation relevanter demographischer Variablen und genereller Zusammenhänge wurde zunächst eine Korrelationsmatrix der hier analysierten Variablen berechnet. Einen Variablenüberblick mit Deskriptiva, Pearsons Korrelationen und Cronbachs Alphas gibt Tabelle 10 auf Seite 150. Daraus ist ersichtlich, dass nur die Skala transformationalen Führungsverhaltens signifikante Zusammenhänge mit demographischen Variablen zeigte. Entsprechend wurden zur Vorhersage des weiten Transfers bezüglich transformationaler Führung neben dem Ausgangswert auch Geschlecht und Hierarchiestufe als Kontrollvariablen in das Regressionsmodell aufgenommen. In Tabelle 11 auf Seite 151 sind die Ergebnisse der hierarchischen Regressionsanalysen zur Erklärung von langfristigem Transferverhalten zusammengefasst. Für alle Kriterien zeigte das Modell 1 bereits eine hohe Varianzaufklärung mit einem korrigierten $\mathrm{R}^{2}$ zwischen $17 \%$ und $43 \%$. Demzufolge sollten die Ausgangswerte der Kriterien und relevante demographische Variablen für aussagekräftige Ergebnisse zur Erklärung von Transfer unbedingt berücksichtigt werden. Für das zweite Modell der Regressionsanalysen wurden verschiedene Alternativen 
berechnet, um zu prüfen, ob die Modellannahme der Hypothese 2 eine bessere Vorhersagekraft besitzt als andere Annahmen aus aktueller Transferforschung. 


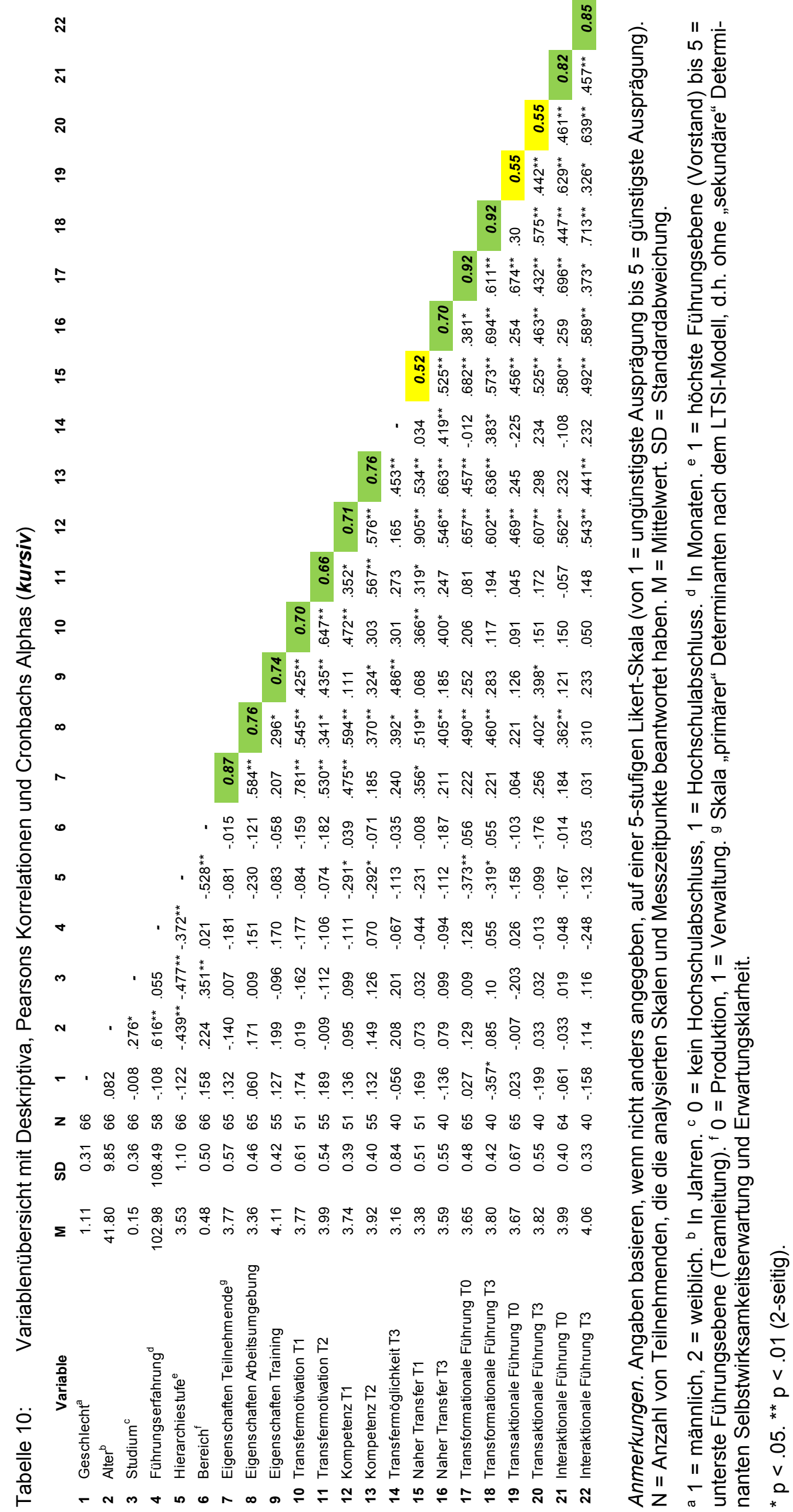




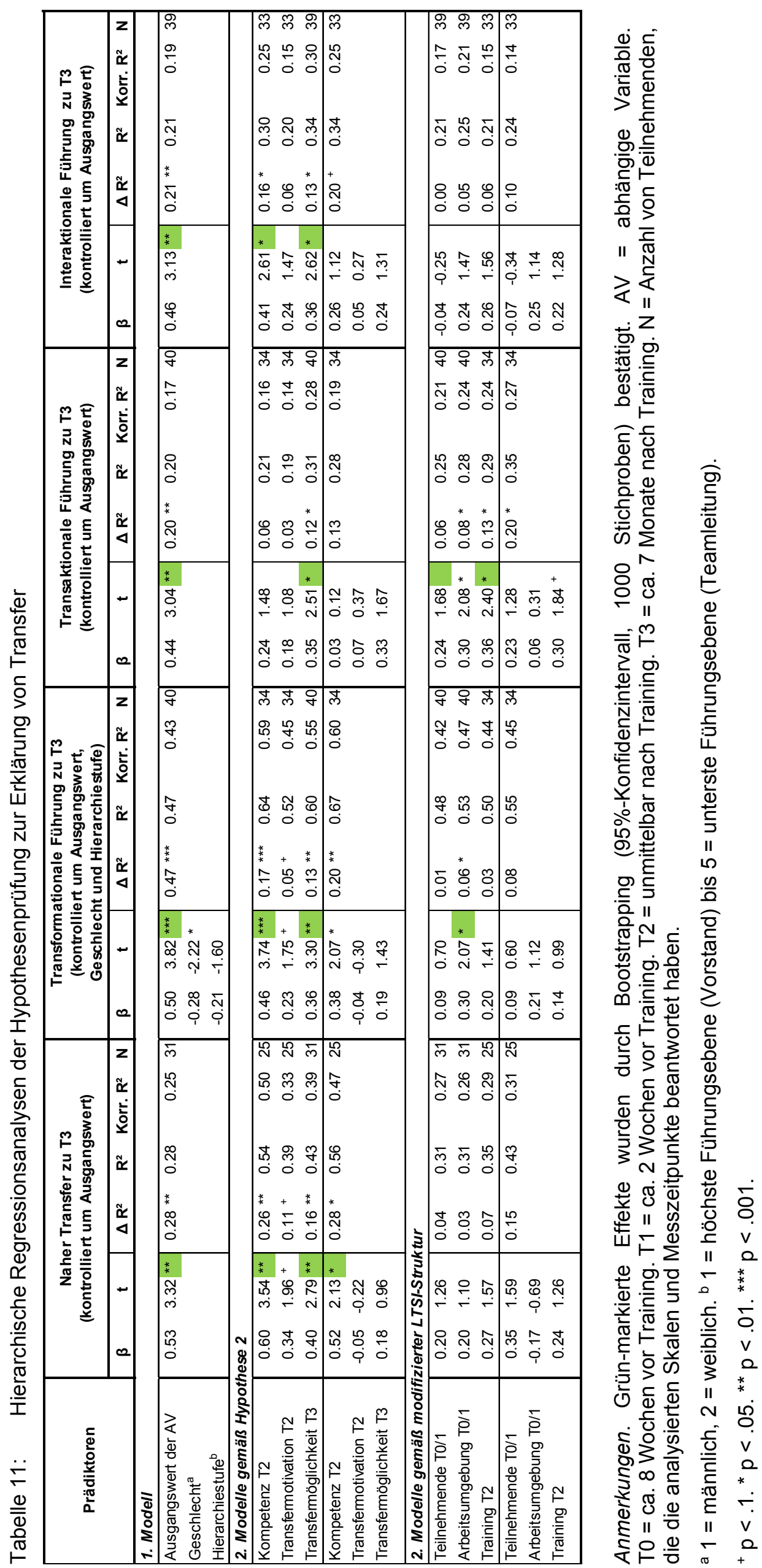


Gemäß Hypothese 2 wurde zunächst die Vorhersagekraft der angenommenen primären Transferdeterminanten (a) Kompetenz, (b) Transfermotivation und (c) -möglichkeit in Einzelanalysen überprüft. Diese Transferdeterminanten klärten dabei zwischen 5\% und $26 \%$ zusätzliche Varianz $(p<.1)$ des Transfers über Ausgangswert und relevante demographische Variablen hinaus auf. Die Ergebnisse zeigten, dass Kompetenz (allerdings mit Ausnahme der nicht signifikanten Vorhersagekraft für transaktionale Führung) und Transfermöglichkeit (für alle Transferkriterien) eine signifikante zusätzliche Vorhersagekraft über Ausgangswerte und demographische Variablen hinaus besitzen. Bei Kompetenz waren Beta-Werte und zusätzlich aufgeklärte Varianz generell höher als bei Transfermöglichkeit. Einzige Ausnahme war die Vorhersage transaktionaler Führung. Allerdings kann diese Skala aufgrund der suboptimalen internen Konsistenz nur bedingt als belastbares Kriterium gelten. Transfermotivation zeigte dagegen nur marginal signifikante Vorhersagekraft für nahen Transfer und transformationale Führung. Allerdings wurden die Effekte bezüglich transaktionaler und interaktionaler Führung nicht signifikant. Dennoch weisen die Beta-Werte auch für diese Transferkriterien auf deutliche positive Zusammenhänge hin, die bei einer größeren Stichprobe auch signifikant sein könnten. Zur Überprüfung von Hypothese 2 (d) wurden Beta-Werte und Varianzaufklärung der drei Transferdeterminanten verglichen, wobei sich zeigte, dass Kompetenz bei drei der vier Transferkriterien die beste Vorhersagekraft besitzt. Nur bei transaktionaler Führung zeigte die Transfermöglichkeit einen größeren Transfereffekt. Dabei muss angemerkt werden, dass Transfermöglichkeit retrospektiv zusammen mit den Transferkriterien erfasst wurde, während Kompetenz und Transfermotivation ca. 7 Monate früher erhoben wurden. Zwar wurden insbesondere durch die Kontrolle um Ausgangswerte Verzerrungen aufgrund von Common-Method-Bias sowie von sozial erwünschtem Antwortverhalten reduziert, dennoch ist bei Transfermöglichkeit die Wahrscheinlichkeit von Verzerrungen bzw. Überschätzung von Effekten aufgrund von Same-MeasurementContext- bzw. Common-Method deutlich höher als bei den anderen beiden primären Transferdeterminanten. Die trotz des methodischen „Nachteils“ größeren Effekte von Kompetenz im Vergleich zu Effekten der Transfermöglichkeit, stützen daher Hypothese 2 (d) umso mehr.

Um zusätzlich Anhaltspunkte bezüglich relativer bzw. inkrementeller Vorhersagekraft der primären Transferdeterminanten im direkten Vergleich zu erhalten, wurden in einer weiteren Analyse alle drei Prädiktoren gleichzeitig in das zweite Regressionsmodell aufgenommen. Allerdings muss dabei berücksichtigt werden, dass aufgrund der Stichprobengröße bei Regressionsmodellen ab vier Prädiktoren nur noch sehr große Effekte signifikant werden (siehe Abbildung 20 auf Seite 140). Weshalb die bereits beschriebenen Einzelanalysen als aussagekräftiger gelten. Entsprechend konnte dieses Gesamtmodell 
nur für drei der vier Transferkriterien, naher Transfer, transformationale und interaktionale Führung, (marginal) signifikant mehr Varianz aufklären als Ausgangswerte relevante demographische Variablen bzw. Modell 1. Zudem zeigte sich bei nahem Transfer und transformationaler Führung, dass Kompetenz auch kontrolliert um die Einflüsse der anderen Prädiktoren eine signifikante Vorhersagekraft besitzt, was sich bei Transfermotivation und -möglichkeit nicht zeigte.

Um zu überprüfen, ob die gemäß Hypothese 2 angenommenen primären Transferdeterminanten tatsächlich eine bessere Vorhersagekraft liefern als die durch weitere bestätigte Transferdeterminanten ergänzte LTSI-Modellstruktur ${ }^{31}$, wurden zusätzlich Alternativmodelle überprüft. $\mathrm{Zu}$ beachten ist jedoch, dass eine akzeptable Skalengüte der übergeordneten Skala Teilnehmendeneigenschaften erst duch Eliminierung der Subskala „Ergebniserwartung“ erreicht werden konnte (siehe Abschnitt 5.3.5 ab Seite 115). Insofern ist das GLTSI-Modell in der Skala Teilnehmende nicht vollständig berücksichtigt. Auch hier wurden zunächst in Einzelanalysen die als direkte Transferdeterminanten bestätigte Variablen ${ }^{32}$ anhand der übergeordneten Skalen Eigenschaften der Teilnehmenden, der Arbeitsumgebung und des Trainings in das zweite Regressionsmodell aufgenommen. Für nahen Transfer und interaktionale Führung zeigte sich dabei für keinen Prädiktor eine signifikante Vorhersagekraft. Für transformationale Führung zeigte sich nur bei transferförderlicher Arbeitsumgebung ein signifikanter Transfereffekt, wobei die Beta-Werte und Varianzaufklärung von Kompetenz und Transfermöglichkeit auch für dieses Transferkriterium deutlich höher waren. Lediglich transaktionale Führung konnte durch Arbeitsumgebung und Training ähnlich gut vorhergesagt werden, wie durch Transfermöglichkeit, wobei diese dennoch eine höhere zusätzliche Varianzaufklärung im Vergleich zu Arbeitsumgebung zeigte. Entsprechend besaß auch das Gesamtmodell, in dem die Prädiktoren der erweiterten LTSI-Struktur gleichzeitig berücksichtigt wurden, nur für transaktionale Führung zusätzliche Varianzaufklärung, die signifikant und sogar etwas größer als das Hypothesenmodell primärer Transferdeterminanten war. Ansonsten besaß das Hypothesenmodell bei gleicher Stichprobengröße und Prädiktorzahl bessere Vorhersagekraft. Dabei zeigte sich im Gesamtmodell der LTSIStruktur auch nur die transferförderliche Trainingsgestaltung einen marginal signifikanten inkrementellen Transfereffekt für transaktionale Führung. Allerdings muss auch bei diesem Gesamtmodell berücksichtigt werden, dass aufgrund der Stichprobengröße nur sehr große Effekte signifikant werden (siehe Abbildung 20 auf Seite 140). Zudem besteht

31 Diese gilt als „State of the Art" der Transferforschung (siehe Abschnitt 3.2.2 ab Seite 76).

32 Bei Berücksichtigung der nach LTSI „sekundären“ Transferdeterminanten (Selbstwirksamkeitserwartung und Erwartungsklarheit) ergeben die Analysen ein gleichwertiges Ergebnismuster. 
eine Einschränkung darin, dass auch die Items der LTSI-Struktur selbstkonstruiert waren.

Analysen mit „vollständigen“ Datensätzen, bei denen fehlende Werte durch Skalenmittelwerte ersetzt wurden, bestätigten die Ergebnisse des Originaldatensatzes weitestgehend. Abweichungen ergaben sich insbesondere bezüglich inkrementeller Vorhersagekraft von Transfermöglichkeit im Gesamtmodell der Hypothese 2, die bei allen vier Transferkriterien signifikant war. Das deutet darauf hin, dass der Originaldatensatz aufgrund der Stichprobengröße zu wenig statistische Power besitzt, um diesen Effekt bestätigen zu können und das diesbezüglich ein Betafehler wahrscheinlich ist. Allerdings sind die Modellgütekriterien des vervollständigten Datensatzes generell schlechter ${ }^{33}$, was dafür spricht trotzt Stichprogengröße und fehlenden Werten den Originaldatensatz zu verwenden. Um die statistische Power zu erhöhen wurden daher Bootstrapping-Analysen der Koeffizienten durchgeführt, die die Ergebnisse bestätigten (abgesehen von den marginal signifikanten Effekten).

Insgesamt bestätigen die Ergebnisse von mindestens drei der vier Transferkriterien Hypothese 2 (a), (c) und (d). Bei transaktionaler Führung konnte nur Hypothese 2 (c) bestätigt werden. Allerdings könnte dies auch durch die suboptimale interne Konsistenz dieser Skala bedingt sein. Hypothese 2 (b) bezüglich Vorhersagekraft der Transfermotivation, konnte dagegen nur teilweise durch marginal signifikante Transfereffekte bei nahem Transfer und transformationaler Führung bestätigt werden. Bei den distaleren Transferkriterien transaktionale und interaktionale Führung zeigten sich zwar BetaWerte von .18 und .24, die jedoch für signifikante Transfereffekte der Transfermotivation nicht ausreichten. Eine Erklärung könnte neben der Stichprobengröße insbesondere der Messzeitpunkt unmittelbar nach dem Training sein: Aktuelle Forschungsbefunde weisen darauf hin, dass für langfristigen Transfer die Entwicklung der Transfermotivation über die Transferzeit hinweg entscheidend ist, wobei diese von Konsequenzen erster Transferversuche gemäß eines dynamischen Interaktionismus zwischen Situation und Person beeinflusst wird (Blume et al., 2019). Dennoch konnte für alle Transferkriterien zusätzliche Varianz durch die angenommenen primären Transferdeterminanten aufgeklärt werden - signifikant über den bereits substanziellen Aufklärungsraten von Ausgangswerten und relevanten demographischen Variablen: $28 \%$ für nahen Transfer (korr. $\mathrm{R}^{2}$ einschließlich 1. Modell $=47 \%$ ), 20\% für transformationale Führung (korr. $\mathrm{R}^{2}$ einschließlich 1. Modell $=60 \%$ ), 12\% für transaktionale Führung (nur durch Transfermöglichkeit, da das Gesamtmodell keinen signifikanten Effekt zeigte, korr. $R^{2}$ einschließlich 1. Modell =

${ }^{33}$ Dieses Problem zeigt sich bei sämtlichen imputationsbasierten Verfahren, auch bei multipler Imputation. 
$28 \%$ ) und $20 \%$ für interaktionale Führung (korr. $R^{2}$ einschließlich 1 . Modell $=25 \%$ ). Studien in denen Transfer mit den 16 validierten LTSI-Variablen erklärt wurde, zeigten Varianzaufklärungen zwischen 49\% und 80\% (Kauffeld et al., 2008; Yaghi, Goodman, Holton \& Bates, 2008; Zamani et al., 2016). Entsprechend kann die hier erreichte Varianzaufklärung der Transfervariablen zwischen $25 \%$ und $60 \%$ basierend auf Hypothese 2 durch nur vier bis sechs Variablen als relativ gut bewertet werden. Berücksichtigt man zudem, dass bei der vorliegenden Stichprobengröße nur sehr große Effekte signifikant werden, können die Ergebnisse, die Hypothese 2 bestätigen, auch als substanziell und aussagekräftig gelten.

\section{Explorative Analysen}

Für einen Überblick über die einzelnen Transfereffekte aller potenziellen sekundären Transferdeterminanten, wurden die LTSI-Skalen sowie die ergänzten Skalen Selbstwertschätzung, Lernmotivation und Trainingsmotivation basierend auf hierarchischen Regressionen explorativ in Einzelanalysen untersucht. Auch diese Analysen berücksichtigten Ausgangswerte und relevante demographische Variablen im ersten Modell. Nur so konnte geprüft werden, ob Transferdeterminanten einen Transfereffekt zeigen, der nicht bereits durch Basiseigenschaften der Teilnehmenden erklärt werden könnte. Die Ergebnisse der explorativen Analysen zur Erklärung von Transfer sind in Tabelle 12 auf Seite 156 zusammengefasst. Zu berücksichtigen ist, dass aufgrund suboptimaler interner Konsistenz bei den Skalen Kollegenunterstützung und Feedback zu T0 sowie Transferdesign und Anwendungsmöglichkeit zu T2 mögliche Effekte unterschätzt bzw. nicht identifiziert werden könnten. 


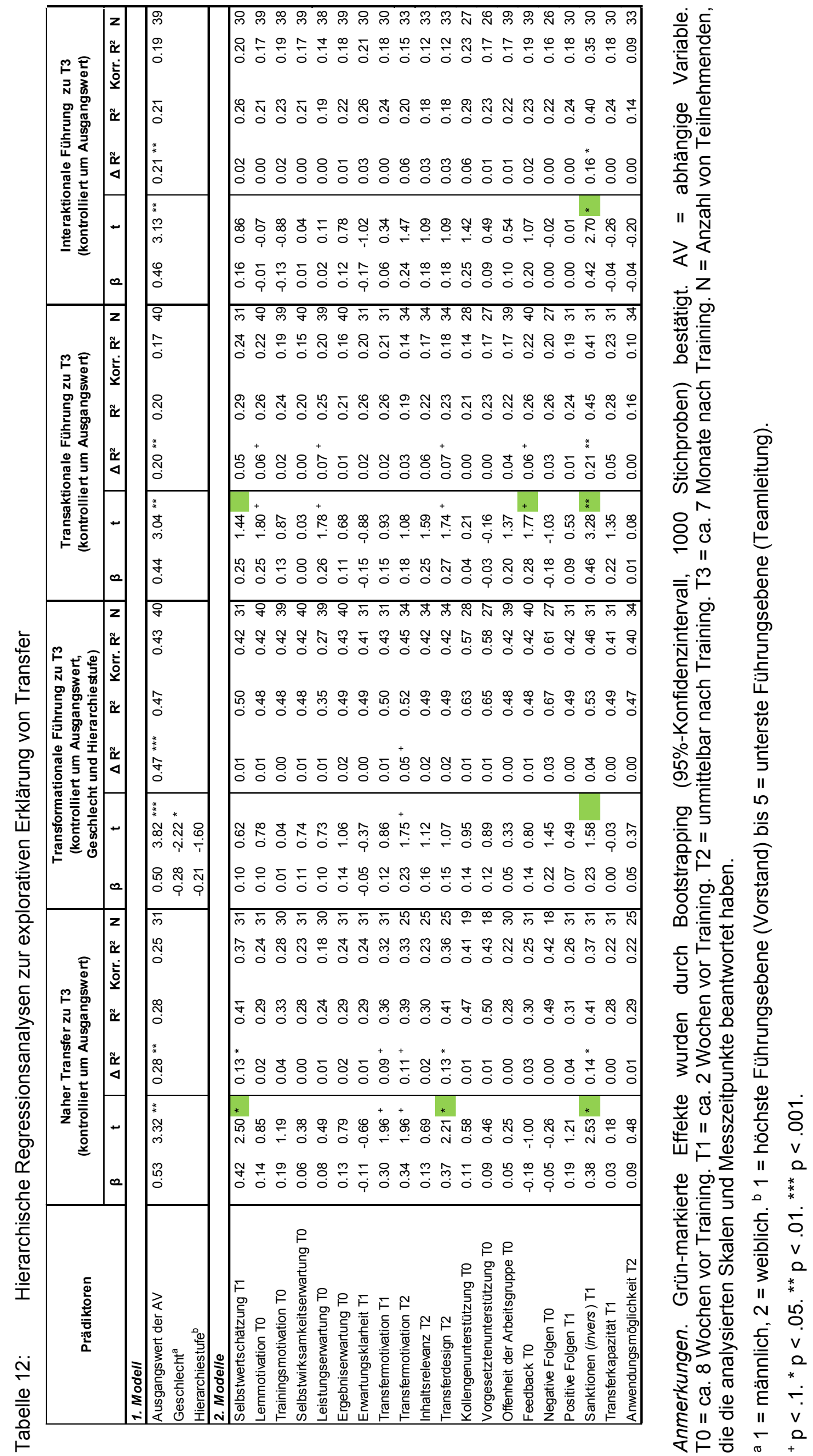


Die Analysen zeigten nur für die Transferdeterminante "Sanktionen" (invers) ein relativ konsistentes Bild über drei der vier Transferkriterien hinweg mit einer signifikanten zusätzlichen Varianzaufklärung zwischen $14 \%$ und $21 \%$. Nur bei transformationaler Führung konnte kein signifikanter Effekt nachgewiesen werden, wobei auch hier die Betaund t-Werte relativ hoch waren. Ansonsten zeigte nur noch Transferdesign wiederholt einen Effekt mit zusätzlicher Varianzaufklärung von 13\% und 7\%. Naher Transfer wurde neben diesen beiden Faktoren zusätzlich durch Selbstwertschätzung und Transfermotivation erklärt. Für transformationale Führung konnte, abgesehen von den in Hypothese 2 angenommenen primären Transferdeterminanten, kein weiterer Prädiktor aus den Subskalen identifiziert werden. Während die Hypothesenprüfung für transaktionale Führung nur die primäre Transferdeterminante Transfermöglichkeit in der Transferzeit signifikante Effekte zeigte, wurden für dieses Transferkriterium explorativ die meisten Hinweise auf relevante Prädiktoren identifiziert: Neben Sanktionen und Transferdesign zeigten Lernmotivation, Leistungserwartung und Feedback einen (marginal) signifikanten Transfereffekt bezüglich transaktionaler Führung. Dies ist insofern überraschend, da diese Transferskala die schlechteste interne Konsistenz besitzt. Für interaktionale Führung konnte, neben den bestätigten primären Transferdeterminanten Kompetenz und Transfermöglichkeit der Hypothesenprüfung, wies nur die Subskala erwartete Sanktionen einen Transfereffekt in den explorativen Analysen auf.

Aufgrund der eher inkonsistenten Effekte der LTSI-Transferdeterminanten über die Transferkriterien hinweg (was sich ebenso über verschiedene Studien hinweg auch mit den validierten LTSI-Skalen zeigte, in denen jeweils nur einzelne aber unterschiedliche Determinanten einen Effekt zeigten, siehe auch 3.2 ab Seite 43), kann abgesehen von Sanktionen (und mit vorsichtiger Interpretation Transferdesign) keine weitere Transferdeterminante als genereller Transferprädiktor identifiziert werden. Allerdings muss berücksichtigt werden, dass auch die LTSI-Skalen für diese Studie selbstkonstruiert wurden und nicht die validierten Items verwendet werden konnten. Die Skala Ergebniserwartung zeigte bei der Erklärung transformationaler Führung etwas mehr als $5 \%$ der Fälle mit standardisierten Residuen über 2, was auf eine suboptimale Modellgüte hinweisen kann. Da jedoch die sonstigen Modellgütekriterien gute Ausprägungen besaßen und $\mathrm{R}^{2}$ nahe an dem korrigierten $\mathrm{R}^{2}$ lag, können die Ergebnisse dennoch als aussagekräftig gelten. Zudem zeigten die Skalen Kollegenunterstützung, Vorgesetztenunterstützung und negative Folgen bei Nicht-Anwendung suboptimale Werte bzw. Muster bei den Voraussetzungen für Regressionsanalysen zur Vorhersage von nahem Transfer bezüglich Homoskedastizität, Linearität und Normalverteilung der Residuen (siehe auch generelle Anmerkungen zu Abschnitt 6.1 ab Seite 139). Daher könnten nichtgefundene Effekte auch in methodischen Problemen begründet liegen werden. 


\subsubsection{Erklärung primärer Transferdeterminanten}

\section{Hypothesenprüfung}

Im Rahmen der prozessorientierten Evaluation wurde zudem Hypothese 3 „Kompetenzentwicklung, Transfermotivation und -möglichkeit können durch transferförderliche (a) Teilnehmendenmerkmale, (b) Arbeitsumgebung und (c) Trainingsgestaltung maßgeblich vorhergesagt werden, (d) wobei Trainingsgestaltung den größten Einfluss zeigt" anhand von Regressionsanalysen überprüft. Einen Variablenüberblick mit Deskriptiva, Pearsons Korrelationen und Cronbachs Alphas gibt Tabelle 10 auf Seite 150. Daraus ist ersichtlich, dass nur die Skala Kompetenz signifikante Zusammenhänge mit der demographischen Variable Hierarchiestufe zeigte. Entsprechend wurde zur Vorhersage von Kompetenzzuwachs neben dem Ausgangswert auch die Hierarchiestufe als Kontrollvariablen in das Regressionsmodell aufgenommen. Eine Abweichung von der Korrelationsmatrix in Tabelle 10 besteht darin, dass für die übergeordnete Skala transferförderlicher Teilnehmendeneigenschaften hier auch die, laut LTSI-Modell, „sekundären“ Subskalen Selbstwirksamkeitserwartung und Erwartungsklarheit berücksichtigt wurden. Die resultierenden Kennwerte der erweiterten Skala sind mit Cronbachs a von .86, einem Mittelwert von 3.77 und einer Standardabweichung von 0.48 jedoch fast identisch mit der reduzierten Skala. Entsprechend sind auch die Ergebnisse beider Skalen im Wesentlichen vergleichbar. Zu beachten ist jedoch, dass eine akzeptable Skalengüte der übergeordneten Skala Teilnehmendeneigenschaften erst duch Eliminierung der Subskala „Ergebniserwartung“ erreicht werden konnte (siehe Abschnitt 5.3.5 ab Seite 115). Insofern ist das GLTSI-Modell in der Skala Teilnehmende nicht vollständig berücksichtigt.

In Tabelle 13 auf Seite 159 sind die Ergebnisse der Regressionsanalysen zur Erklärung primärer Transferdeterminanten zusammengefasst. Für die Kriterien, für die Ausgangswerte erfasst bzw. kontrolliert wurden, zeigte das Modell 1 bereits eine hohe Varianzaufklärung mit einem korrigierten $\mathrm{R}^{2}$ von $31 \%$ bei Kompetenz und $40 \%$ bei Transfermotivation. Demzufolge sollten die Ausgangswerte der Kriterien und relevante demographische Variablen für aussagekräftige Ergebnisse auch zur Erklärung dieser Transferdeterminanten berücksichtigt werden. 


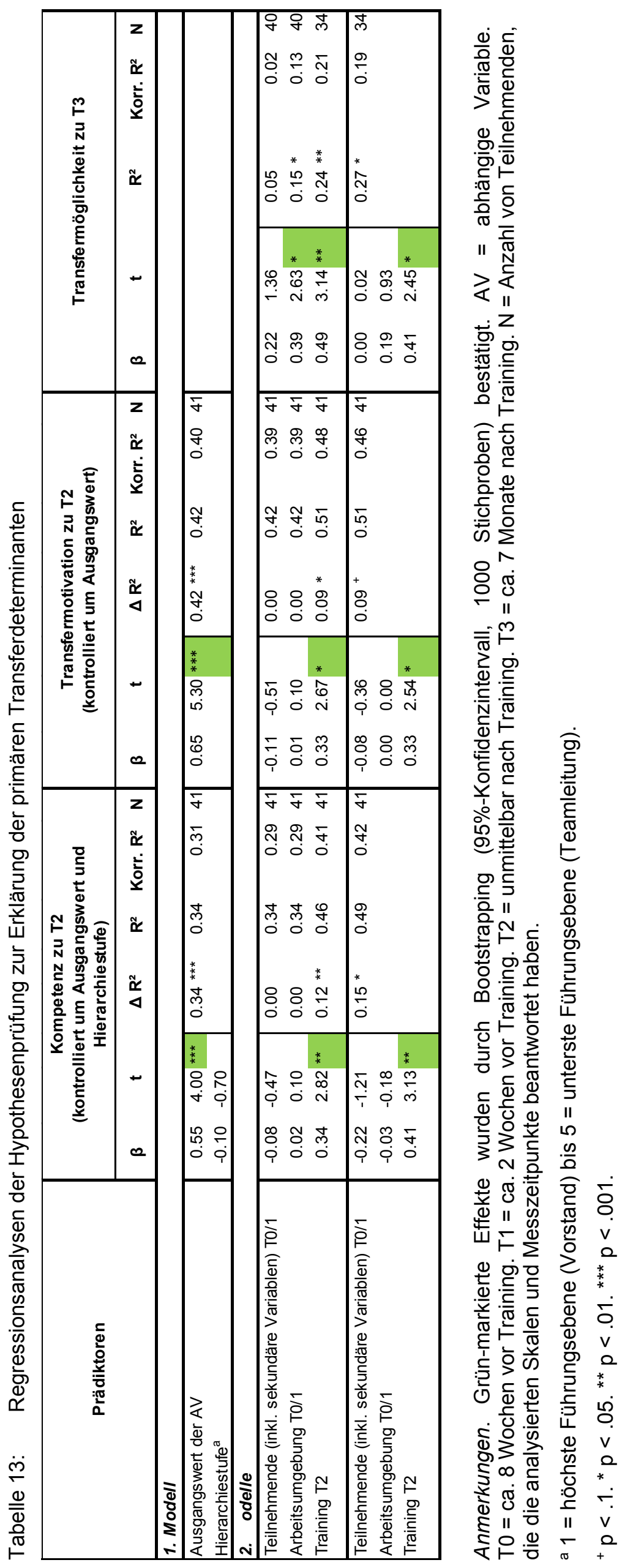


Gemäß Hypothese 3 wurde zunächst die Vorhersagekraft der übergeordneten Einflussbereiche (a) Teilnehmendenmerkmale, (b) Arbeitsumgebung und (c) Trainingsgestaltung in Einzelanalysen überprüft. Diese Transferdeterminanten klärten dabei zwischen $9 \%$ und $24 \%$ zusätzliche Varianz ( $p$ < .05) der primären Transferdeterminanten auf (ggf. über Ausgangswert und relevante demographische Variablen hinaus). Dabei zeigte nur die Bewertung der Trainingsgestaltung für alle drei Transferdeterminanten signifikante Effekte. Für die situative Transferdeterminante Transfermöglichkeit, besaß auch die transferförderliche Arbeitsumgebung signifikante Vorhersagekraft, wobei Beta-Werte und aufgeklärte Varianz kleiner waren als bei den Effekten der Trainingsgestaltung. Für transferförderliche Teilnehmendenmerkmale konnte dagegen kein Effekt auf die primären Transferdeterminanten nachgewiesen werden.

Um zusätzlich Anhaltspunkte bezüglich relativer bzw. inkrementeller Vorhersagekraft der drei Einflussfaktoren im direkten Vergleich zu erhalten, wurden in einer weiteren Analyse alle drei Prädiktoren gleichzeitig in das zweite Regressionsmodell aufgenommen. Allerdings muss dabei berücksichtigt werden, dass aufgrund der Stichprobengröße bei Regressionsmodellen ab vier Prädiktoren nur noch sehr große Effekte signifikant werden (siehe Abbildung 20 auf Seite 140). Dennoch konnte dieses Gesamtmodell für alle drei primären Transferdeterminanten (marginal) signifikant mehr Varianz aufklären als Ausgangswerte und relevante demographische Variablen bzw. Modell 1. Basierend auf den korrigierten $\mathrm{R}^{2}$-Werten konnte das Gesamtmodell sekundärer Transferdeterminanten für Kompetenz 42\%, für Transfermotivation 46\% und für Transfermöglichkeit 19\% der Varianz aufklären. Zudem blieb die Vorhersagekraft der transferförderliche Trainingsgestaltung auch kontrolliert um die Einflüsse der anderen Prädiktoren signifikant, was sich bei dem Effekt der Arbeitsumgebung auf Transfermöglichkeit nicht zeigte.

Von den drei übergeordneten Transferfaktoren transferförderliche Teilnehmendenmerkmale, Arbeitsumgebung und Trainingsgestaltung scheint Trainingsgestaltung für die Vorhersage und Förderung der zentralen Transferdeterminanten und dadurch vermittelt für Transfererfolg am wichtigsten zu sein. Allerdings muss berücksichtigt werden, dass die Erfassung der Trainingsgestaltung unmittelbar nach dem Training erfolgte, während die anderen beiden Faktoren einige Wochen vor dem Training erhoben wurden. Die Ergebnisse jedoch nur auf einen Same-Measurement-Context- bzw. Common-Method-Bias zurück zu führen wäre insofern jedoch unplausibel, da sich die Effekte nicht nur in den zum selben Messzeitpunkt erfassten Variablen zeigte, sondern in vergleichbarer Höhe auch bei Transfermöglichkeit, die ca. 7 Monate später erfasst wurde. Gerade bei dieser situativen Variable zeigte sich zudem ein signifikanter Effekt der Arbeitsumgebung (was inhaltlich plausibel ist), obwohl die Messzeitpunkte hier noch weiter auseinander lagen. Zudem wurden Kompetenz und Transfermotivation um ihre Ausgangswerte kontrolliert, 
um dem Common-Method-Bias sowie Verzerrungen aufgrund sozial erwünschtem Antwortverhalten entgegenzuwirken. Daher weisen die Ergebnisse eher auf inhaltliche Zusammenhänge hin als auf rein methodische Artefakte. Darüber hinaus wurden alle signifikanten Ergebnisse auch durch Bootstrapping-Analysen bestätigt, was auf robuste Effekte hinweist.

Insgesamt können daher Hypothese 3 (c) und (d) für alle drei primären Transferdeterminanten Kompetenz, Transfermotivation und -möglichkeit als bestätigt gelten. Hypothese 3 (b) wurde nur für Transfermöglichkeit gestützt, während Hypothese 3 (a) bezüglich der Vorhersagekraft sekundärer Teilnehmendeneigenschaften für keine primäre Transferdeterminante bestätigt werden konnte. Allerdings muss berücksichtigt werden, dass die LTSI-Skalen für diese Studie selbstkonstruiert wurden und nicht die validierten Items verwendet werden konnten.

\section{Explorative Analysen}

Für einen Überblick über die Effekte aller potenziellen sekundären Transferdeterminanten auf Kompetenzzuwachs, Transfermotivation und -möglichkeit, wurden die einzelnen LTSI-Skalen sowie die ergänzten Skalen Selbstwertschätzung, Lernmotivation und Trainingsmotivation basierend auf Regressionen explorativ analysiert. Auch diese Analysen berücksichtigten, soweit vorhanden, Ausgangswerte und relevante demographische Variablen im ersten Modell. Die Ergebnisse der explorativen Analysen zur Erklärung primärer Transferdeterminanten sind in Tabelle 14 auf Seite 162 zusammengefasst. Allerdings muss auch hier berücksichtigt werden, dass die LTSI-Skalen für diese Studie selbstkonstruiert wurden und nicht die validierten Items verwendet werden konnten. Entsprechend sind die Ergebnisse der Subskalen nicht direkt mit Studien vergleichbar, die die Originalitems des LTSI verwendet haben. Zudem könnten aufgrund suboptimaler interner Konsistenz bei den Skalen Kollegenunterstützung und Feedback zu T0 sowie Transferdesign und Anwendungsmöglichkeit zu T2 mögliche Effekte unterschätzt bzw. nicht identifiziert werden. 


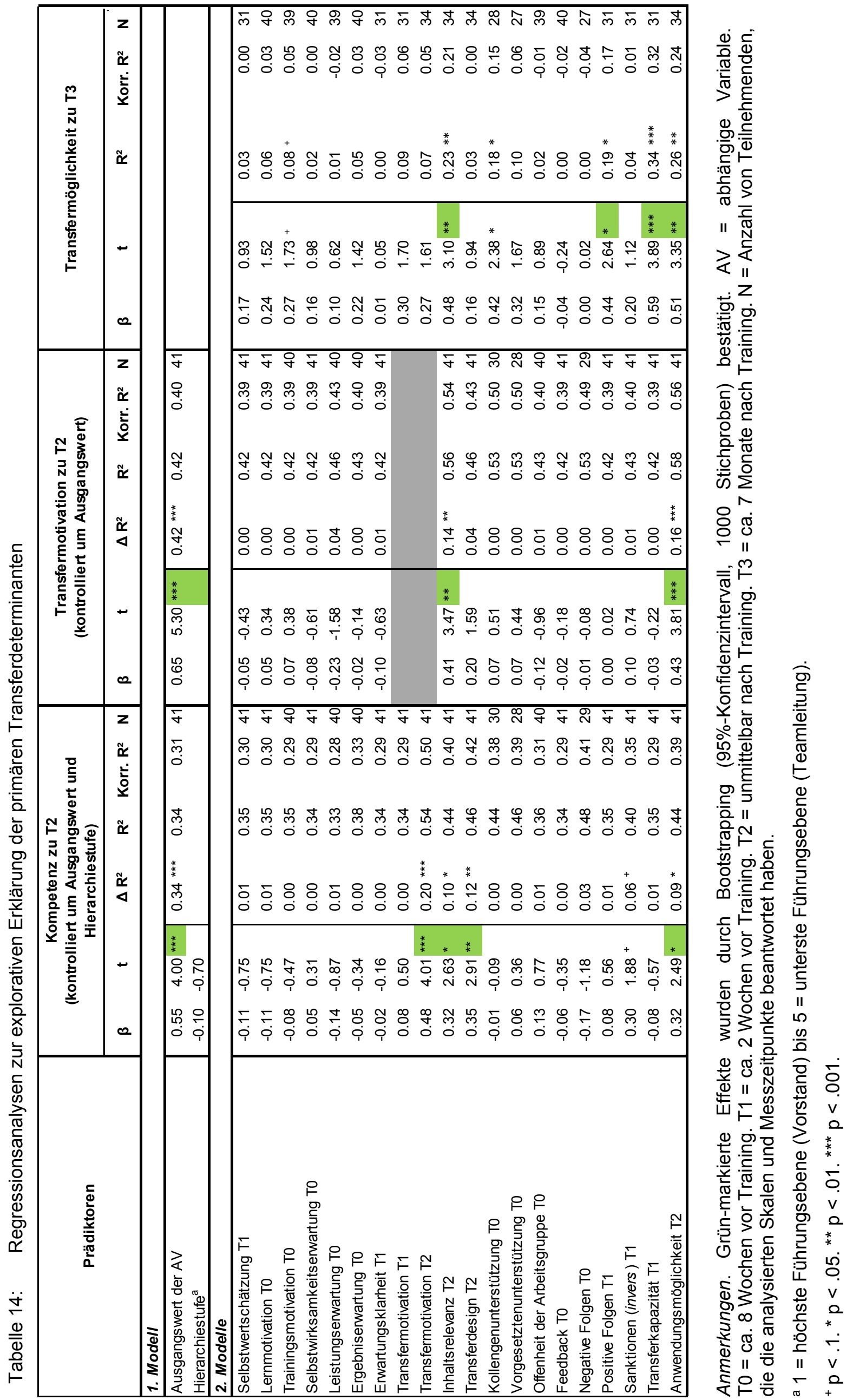


Die Analysen zeigten nur für die Skalen Inhaltsrelevanz (mit einer signifikanten zusätzlichen Varianzaufklärung zwischen $10 \%$ und $23 \%$ ) und erwartete Anwendungsmöglichkeit (mit einer signifikanten zusätzlichen Varianzaufklärung zwischen 9\% und 26\%) ein konsistentes Bild über alle drei primären Transferdeterminanten hinweg. Kompetenzentwicklung wurde neben diesen beiden Faktoren zusätzlich durch Transfermotivation unmittelbar nach Training (mit einer sehr hohen zusätzlichen Varianzaufklärung von 20\%) sowie durch die Variablen Transferdesign und Sanktionen (invers), die bereits als relevante Prädiktoren der Transferkriterien identifiziert wurden, erklärt. Dagegen konnten für Transfermotivation nach Kontrolle des Ausgangswertes (abgesehen von Inhaltsrelevanz und erwartete Anwendungsmöglichkeit, siehe oben) keine weiteren Prädiktoren identifiziert werden. Allerdings zeigten weitere Analysen, dass ohne Kontrolle des Ausganswertes alle drei übergeordneten Skalen Transfermotivation nach dem Training signifikant vorhersagen (siehe Tabelle 15 auf Seite 165). Auch ein Großteil ihrer Subskalen zeigte signifikante Effekte zur Erklärung von Transfermotivation, die nach Kontrolle des Ausgangswertes nicht mehr existierten ${ }^{34}$. Daraus kann geschlossen werden, dass Eigenschaften von Teilnehmenden und Arbeitsumgebung vermittelt über Transfermotivation vor dem Training auf die Transfermotivation nach dem Training wirken. Die Gegenüberstellung auf Subskalenniveau weist auf folgende indirekte Prädiktoren hin: 1) Lernmotivation, Trainingsmotivation und Erwartungsklarheit als Teilnehmendeneigenschaften sowie 2) Vorgesetztenunterstützung, Erwartung negativer Folgen bei Nicht-Anwendung bzw. positiver Folgen und Sanktionen (invers) bei Anwendung und Transferkapazität als Eigenschaften der Arbeitsumgebung. Transfermöglichkeit wurde neben Inhaltsrelevanz und erwarteter Anwendungsmöglichkeit durch Trainingsmotivation und Faktoren der Arbeitsumgebung (Kollegenunterstützung ${ }^{35}$, positive Folgen bei Anwendung sowie Transferkapazität) unmittelbar vor und nach dem Training vorhergesagt. Die Skalen Leistungserwartung, Erwartungsklarheit, Offenheit der Arbeitsgruppe, Feedback und negative Folgen bei Nicht-Anwendung zeigten suboptimale Werte bzw. Muster bei den Voraussetzungen für Regressionsanalysen zur Vorhersage von Transfermöglichkeit bezüglich Homoskedastizität, Linearität und Normalverteilung der Residuen (siehe auch generelle Anmerkungen zu Abschnitt 6.1 ab Seite 139). Daher könnten nichtgefundene Effekte auch in methodischen Problemen begründet liegen werden.

${ }^{34}$ Dies zeigte sich zwar auch bei einer Subskala der Trainingsgestaltung (Transferdesign) allerdings scheint dafür eher eine Reduktion der Stichprobengröße und damit der statistischen Power ursächlich zu sein.

35 Dieses Ergebnis bestätigt bisherige Trainingsforschung (Chauhan et al., 2016; Massenberg et al., 2017; Massenberg et al., 2015; Peters et al., 2014) bezüglich der Annahme, dass Vorgesetztenunterstützung eher relevant für die Förderung von Trainings- und Transfermotivation vor dem Training ist, während Kollegenunterstützung insbesondere für Anwendung nach dem Training relevant ist (siehe Abschnitt .3.2.1 ab Seite 58). 
Aufgrund der eher inkonsistenten Effekte zur Vorhersage der hier als primäre Transferdeterminanten definierten Variablen durch die hier als sekundäre Transferdeterminanten definierten Variablen, können nur Inhaltsrelevanz und erwartete Anwendungsmöglichkeit als generelle Prädiktoren primärer Transferdeterminanten gelten. Darüber hinaus scheinen Kompetenzentwicklung, Transfermotivation und -möglichkeit durch unterschiedliche Wirkmechanismen bedingt zu werden. Die bisherigen Datenanalysen geben dazu Anhaltspunkte, die weiter explorativ untersucht wurden. Aufgrund der Stichprobengröße konnten anhand der bereits beschriebenen Ergebnisse gezielte Teilanalysen der Modellannahme sinnvoll durchgeführt werden. Allerdings konnten weitere mögliche Wechselwirkungen zwischen den Subskalen nicht belastbar überprüft werden. Die Analyseergebnisse sind in den folgenden Ergebnistabellen zusammengefasst (siehe Tabelle 15 bis Tabelle 19 ab Seite 165). 


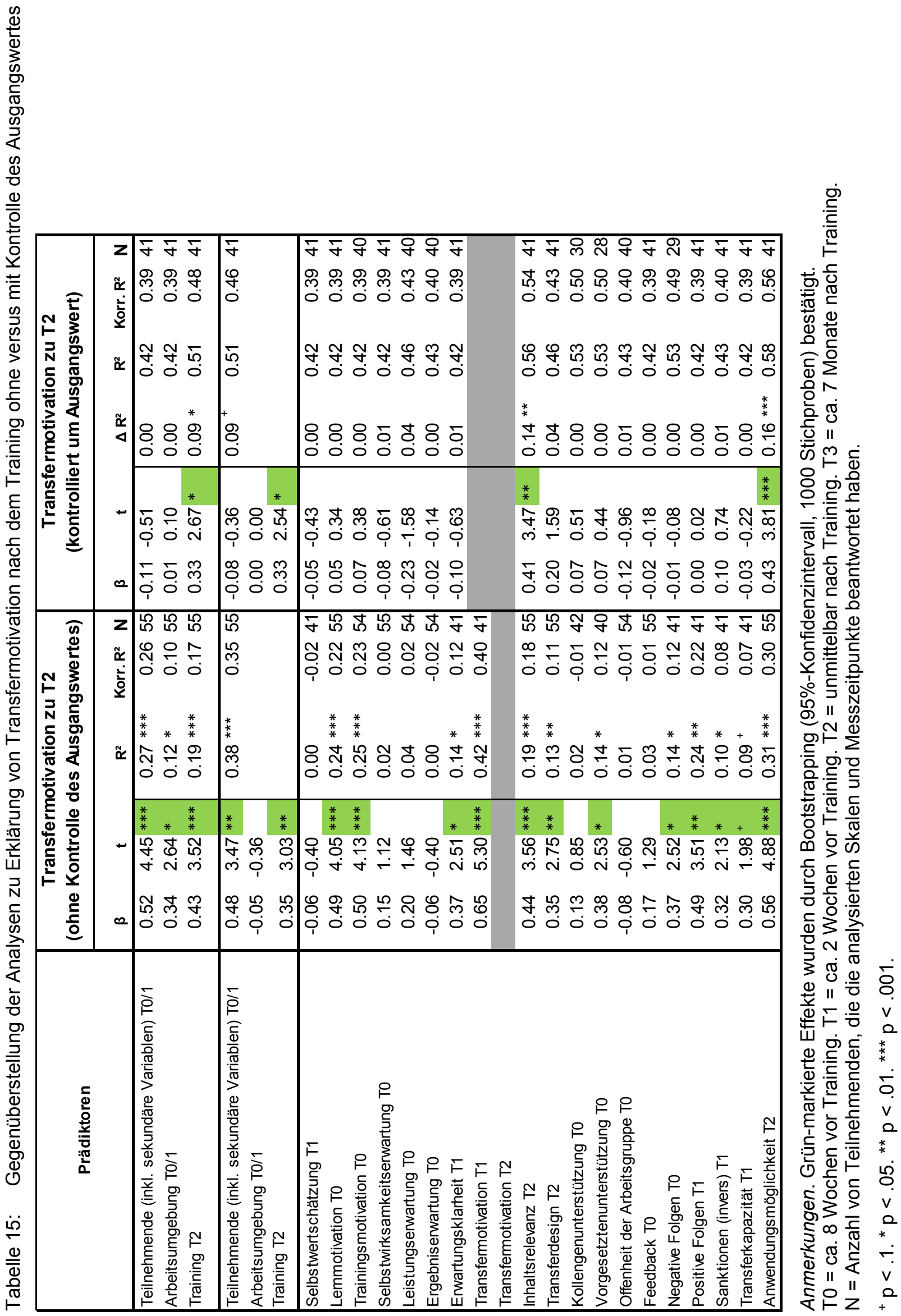




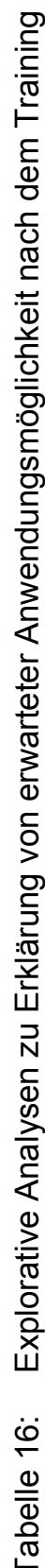

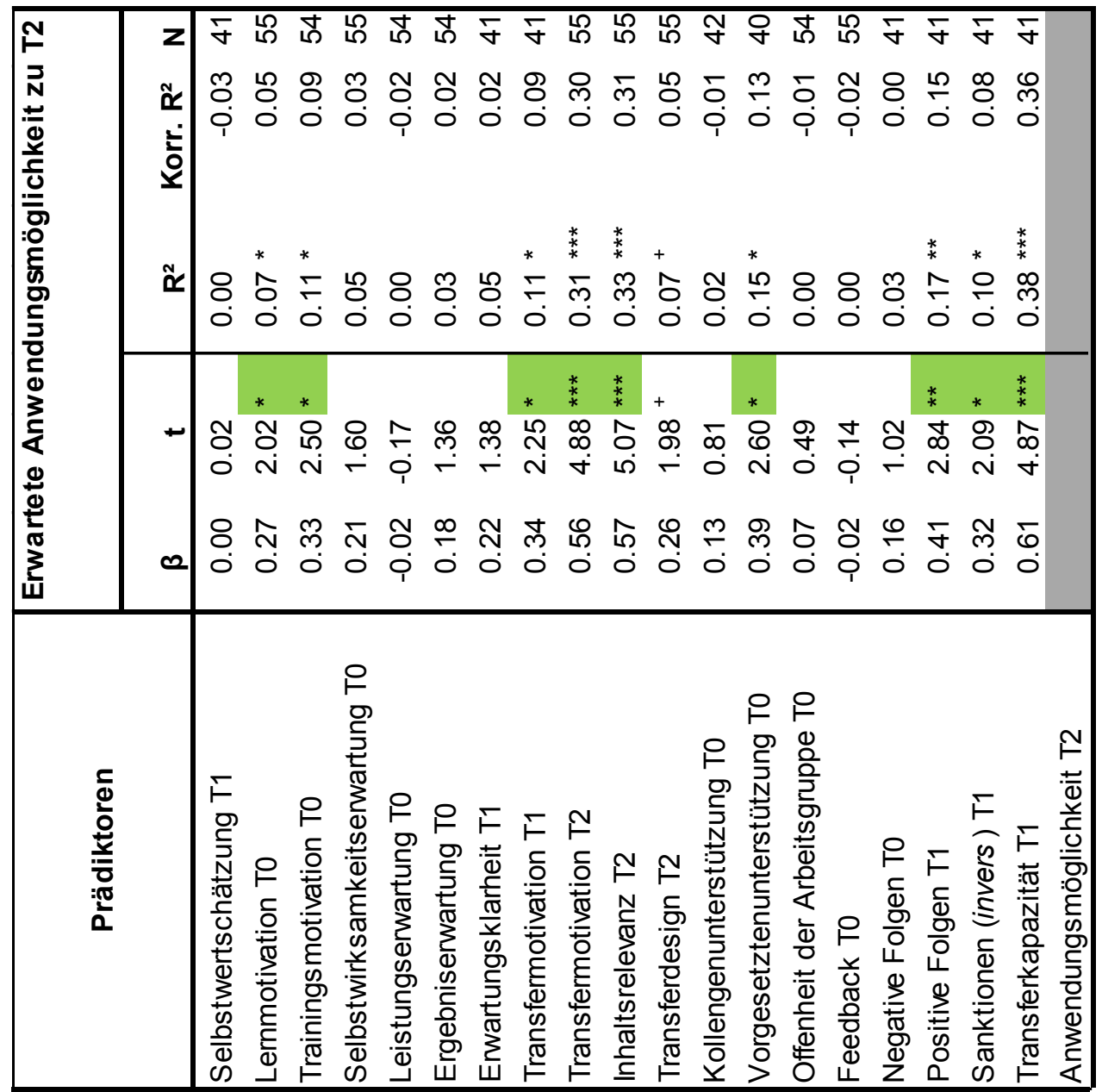

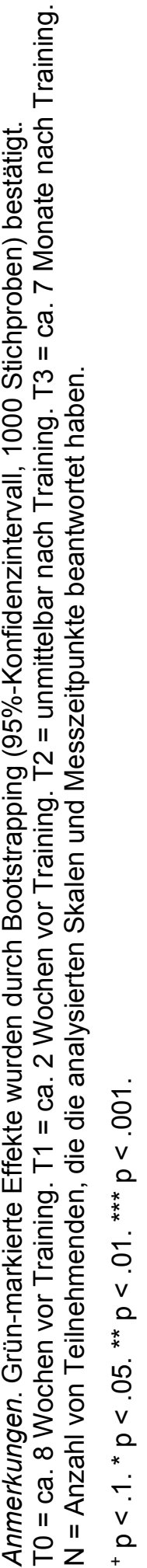



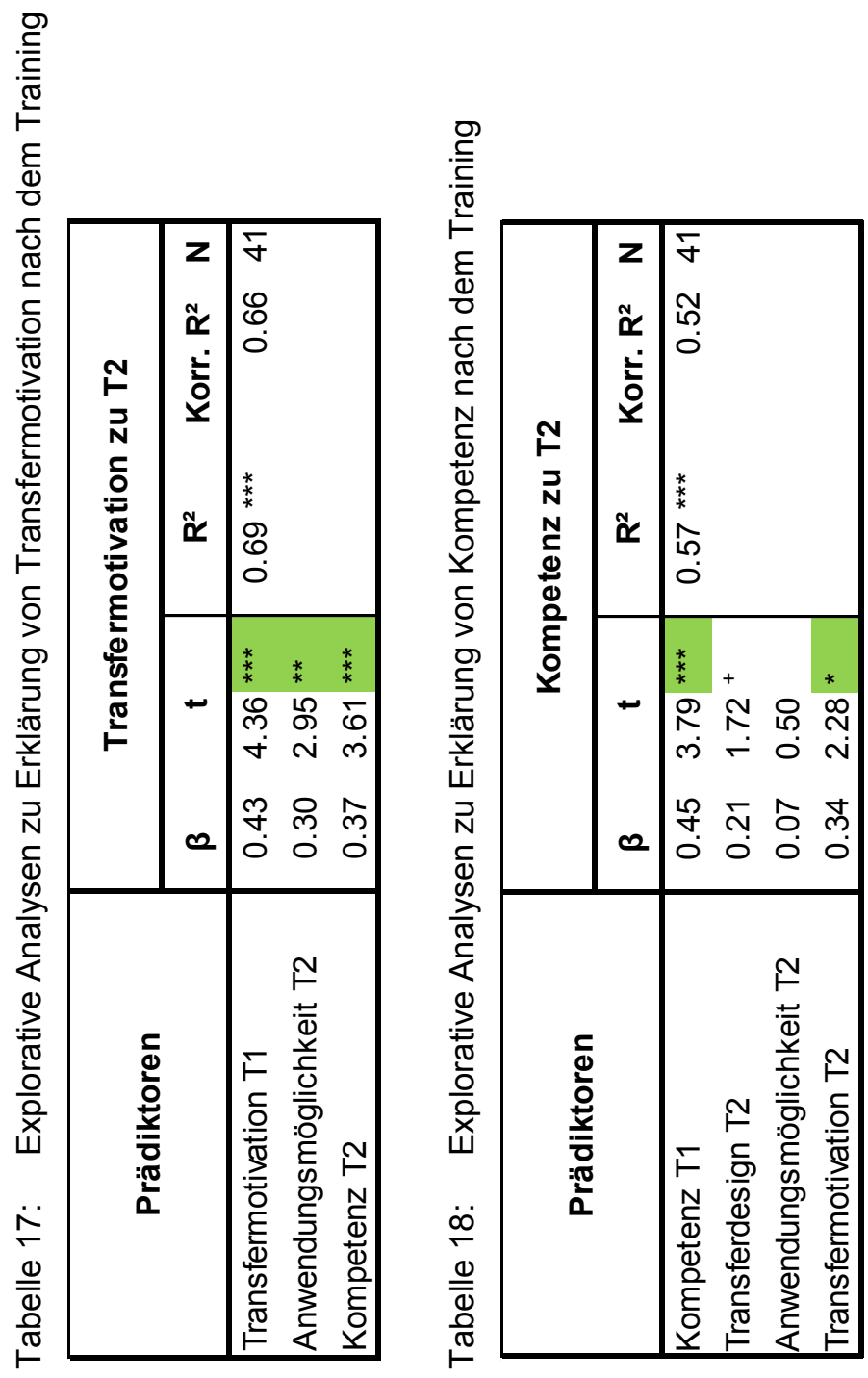

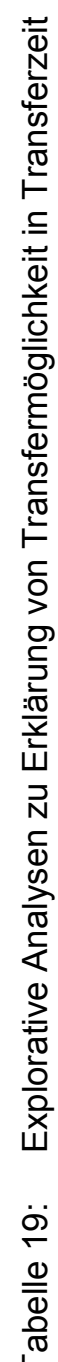

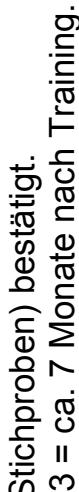

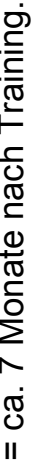

ஸे

용 워

.

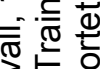

作

중

요

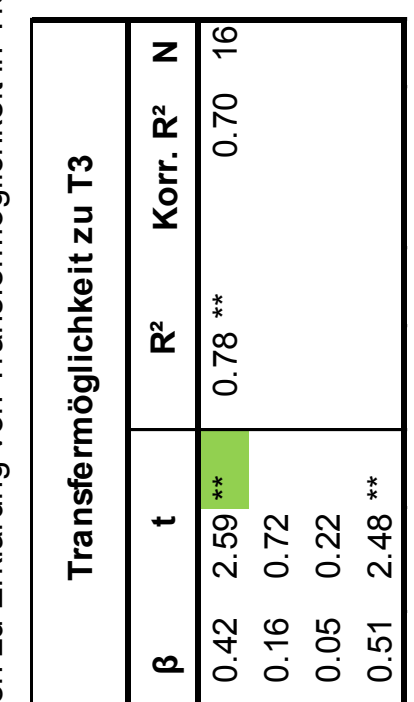

은 은

올

ㅇํㅇ

ถึ 11 N

कㄴ

흥. 하을

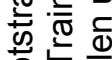

ํำ

के

든 $\frac{\complement}{\Phi}$

की

के

3 ญ $\frac{\pi}{0}$

离占比 v

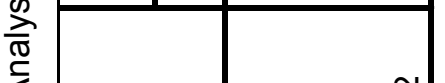

山ें

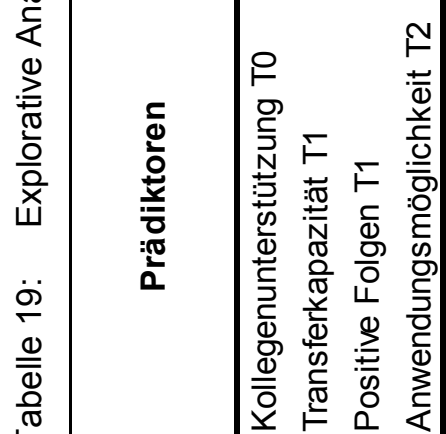

ㄴ.

市

는 $v$

눈

U⿺辶一

ㄷㅇㅇ

$\$ 3 \geq v$

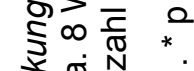

ป ญ

हाII 
Prädiktoren der Transfermotivation vor dem Training wurden aus der bereits beschriebenen Gegenüberstellung abgeleitet (siehe Tabelle 15 auf Seite 165). Daraus lässt sich der Hinweis ableiten, dass insbesondere die Teilnehmendeneigenschaften Lern- und Trainingsmotivation sowie Erwartungsklarheit sowie die Arbeitsumgebungsfaktoren Vorgesetztenunterstützung, Transferkonsequenzen (negative Folgen bei NichtAnwendung bzw. positive Folgen bei Anwendung sowie die invertierte Skala Sanktionen) und Transferkapazität die Transfermotivation bereits vor dem Training bedingen. Zudem zeigte sich in weiteren explorativen Analysen auch ein hochsignifikanter Zusammenhang zwischen Vorwissen bzw. Kompetenz T1 und Transfermotivation zu T1 $(\beta=.472, \mathrm{t}=$ $3.75, p<.001$, siehe auch Korrelation aus Tabelle 10 auf Seite 150). Zur Erklärung der erwarteten Anwendungsmöglichkeit unmittelbar nach dem Training, die sich als zentrale sekundäre Transferdeterminante zeigte (siehe Tabelle 15 auf Seite 165), wurden ebenfalls Regressionsanalysen mit allen früher wirkenden Transferdeterminanten berechnet (siehe Tabelle 16 auf Seite 166). Dabei resultierten als signifikante Prädiktoren die gleichen Arbeitsumgebungsfaktoren, die auch Transfermotivation zu T1 bedingten. Einzige Ausnahme war die Skala negative Folgen bei Nicht-Anwendung, die hier keinen signifikanten Einfluss zeigte. Darüber hinaus zeigten die Trainingseigenschaften einen wichtigen Einfluss, wobei Inhaltsrelevanz eine wesentlich größere Vorhersagekraft aufwies als das Transferdesign, dessen Effekt nur marginal signifikant wurde. Weitere explorative Analysen weisen darauf hin, dass die signifikanten Effekte der Teilnehmendeneigenschaften Lern- und Transfermotivation eher indirekt über Transfermotivation zu T1 auf erwartete Anwendungsmöglichkeit unmittelbar nach dem Training wirken.

Auf der Basis der beschriebenen Analysen wurden in einem letzten Schritt die als zentral identifizierten Variablen in einem Gesamtmodell zur Vorhersage der primären Transferdeterminanten verwendet (siehe Tabelle 17 bis Tabelle 19 auf Seite 167). Demnach konnten 66\% der Varianz der Transfermotivation zu T2 durch Transfermotivation zu T1, erwartete Anwendungsmöglichkeit zu T2 und Kompetenz zu T2 hoch signifikant erklärt werden. 52\% der Varianz der Kompetenz zu T2 konnte (marginal) signifikant durch Kompetenz zu T1, Transferdesign und Transfermotivation zu T2 vorhergesagt werden. Der Effekt der erwarteten Anwendungsmöglichkeit zu T2 (siehe Tabelle 14 auf Seite 162) wurde von den übrigen Variablen überlagert. Für Transfermöglichkeit zu T3 konnten sogar $70 \%$ der Varianz durch die identifizierten Prädiktoren erklärt werden, wobei nur Kollegenunterstützung zu T0 und erwartete Anwendungsmöglichkeit zu T2 einen direkten dafür aber hoch signifikanten Effekt zeigten. Jedoch konnten aufgrund der Kumulation fehlender Werte hier nur 16 Probanden berücksichtigt werden, weshalb diese Er- 
gebnisse nur eine geringe Evidenz besitzen. Allerdings fielen die Voraussetzungsprüfungen der Regressionsanalyse gut aus und auch das $\mathrm{R}^{2}$ liegt mit $78 \%$ nur 8 Prozentpunkte über dem korrigierten $\mathrm{R}^{2}$, was insgesamt auf eine gute Modellpassung hinweist.

Die beschriebenen und als zentral identifizierten Wirkmechanismen der Transferdeterminanten wurden unter Berücksichtigung der zeitlichen Folge und inhaltlichen Logik in Abbildung 21 zusammengefasst.

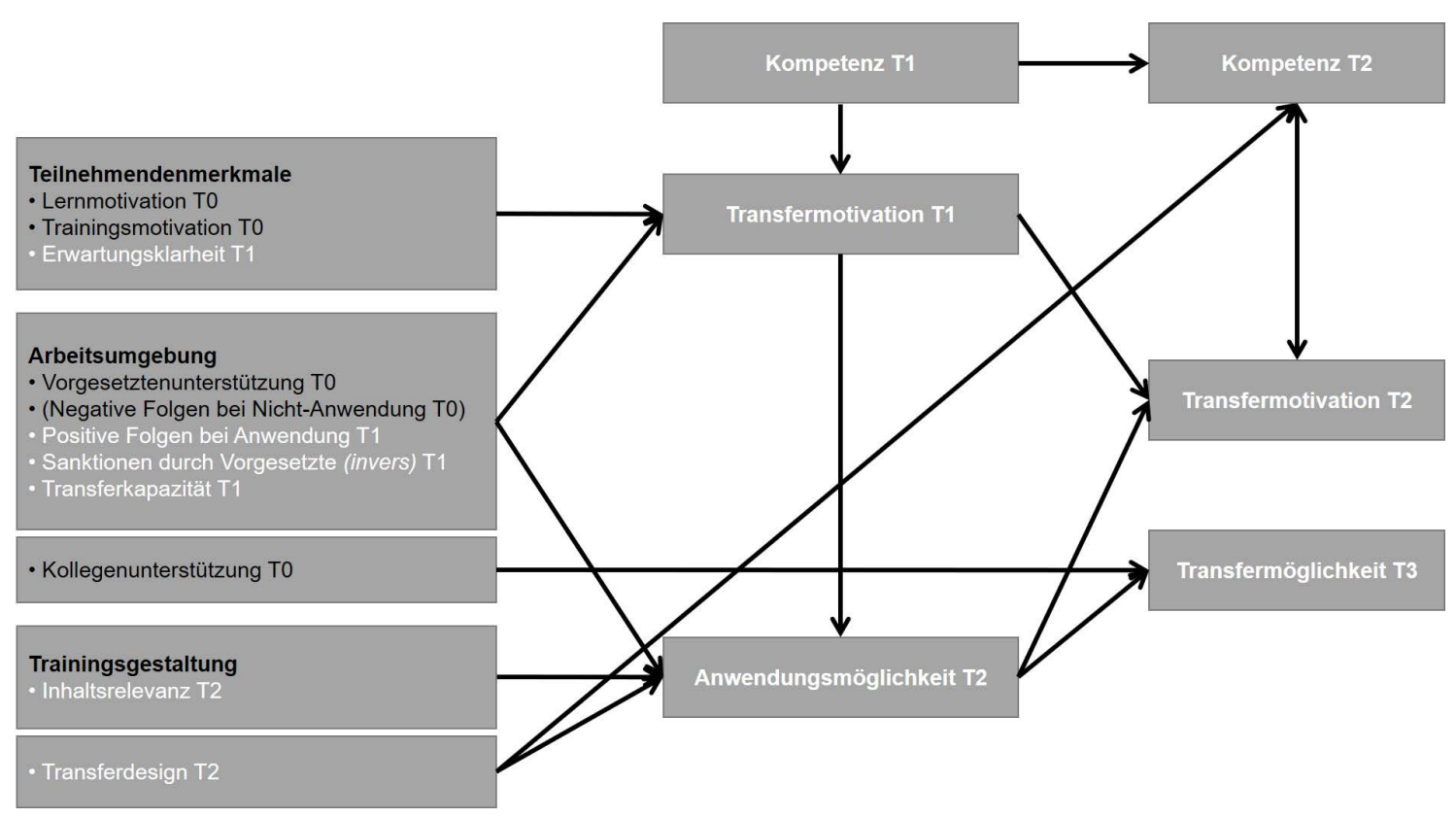

Abbildung 21: Explorative Annahmen zur Erklärung primärer Transferdeterminanten.

Aufgrund der Messzeitpunkte und Datenanalyse kann eine Kausalannahme nicht abgesichert werden. So kann der Zusammenhang zwischen den Variablen auch in umgekehrte oder reziproke Richtung weisen. Insbesondere bei Trainingsgestaltung und erwarteter Anwendungsmöglichkeit könnte dies aufgrund desselben Erhebungszeitpunktes wahrscheinlich sein. Es wird jedoch im vorliegenden Kontext als inhaltlich logischer erachtet, dass eine transferförderliche Trainingsgestaltung die erwartete Anwendungsmöglichkeit bedingt (was per Definition ein Merkmal transferförderlicher Trainingsgestaltung darstellte) als umgekehrt. Auch der Zusammenhang zwischen erwarteter Anwendungsmöglichkeit und Transfermotivation unmittelbar nach dem Training, wurde im selben Fragebogen erfasst. Hier wäre inhaltlich auch ein reziproker Zusammenhang wahrscheinlich, dahingehend, dass gemäß der Definition von Motivation besonders Motivierte Möglichkeiten eher realisieren bzw. dass gemäß Erwartung-X-Wert-Theorien erwartete Anwendungsmöglichkeiten die Motivation erhöhen. Für den Transferprozess scheint jedoch letztlich die Transfermotivation entscheidend zu sein, während die erwartete An- 
wendungsmöglichkeit für kein Transferkriterium einen direkten Effekt zeigte (siehe Tabelle 12 auf Seite 156). Schließlich wurde auch Transfermotivation vor dem Training zusammen mit Erwartungsklarheit, positiven Folgen, Sanktionen und Transferkapazität erfasst. Aber auch hier wird die Kriterienfunktion der Transfermotivation für den Transferprozess inhaltlich und analysebasiert für wahrscheinlicher bzw. entscheidender erachtet. Limitationen der dargestellten Datenanalysen, die bei der Ergebnisinterpretation generell beachtet werden sollten, sind in nachfolgendem Abschnitt zusammengefasst. 


\subsection{Metaevaluation der Wirksamkeitsüberprüfungen}

Zur Überprüfung und kritischen Reflexion des Evaluationsprozesses und dessen Ergebnisse sind in diesem Abschnitt Limitationen der Datenanalyse, Umsetzung der Evaluationsstandards (Nützlichkeit, Durchführbarkeit, Fairness und Genauigkeit) ${ }^{36}$ sowie Handlungsempfehlungen für zukünftige Evaluationen im Sinne einer Metaevaluation zusammengefasst (siehe Abschnitt 2.4 ab Seite 16). Auch wenn versucht wurde, ein bestmögliches Vorgehen zur Überprüfung der Forschungsfragen unter Einhaltung der Evaluationsstandards zu wählen, brachte jede methodische Entscheidung auch Limitationen mit sich. Diese werden nachfolgend basierend auf der Struktur von Kapitel 5 ab Seite 91 Evaluationsdesign, Evaluationsgegenstand und Operationalisierung zusammengefasst und reflektiert.

\subsubsection{Evaluationsdesign}

Übergeordnet handelte es sich beim gewählten Evaluationsdesign um eine Selbstevaluation, da das Trainierteam selbst für die Evaluation verantwortlich war. Dadurch ergeben sich insbesondere Vorteile bezüglich einer Minimierung von Informationsverlusten bzw. einer optimalen Abstimmung zwischen Bedarfsanalyse, Trainingskonzeption, -durchführung und -evaluation sowie prozessbegleitender Transferförderung. Allerdings birgt eine Selbstevaluation verstärkte Gefahr selbstwertdienlicher Hypothesenformulierung und -überprüfung sowie soziale erwünschtes Antwortverhalten (Scriven, 1991). Daher wurde dieser Herausforderung gezielt durch verschiedene methodische Vorgehensweisen begegnet, um derartige Effekte zu minimieren. Einerseits wurde der Gefahr selbstwertdienlicher Hypothesenformulierung begegnet, indem Hypothesen und betrachtete Variablen ganzheitlich aus dem aktuellen Forschungsstand abgeleitet wurden, um selektive oder willkürliche Hypothesenprüfungen auszuschließen. Andererseits wurden Evaluationskriterien zum Trainings- und Transfererfolg vorab von einer umfassenden Bedarfsanalyse abgeleitet. Ihre konkrete Operationalisierung wurde in Abstimmung mit dem Unternehmen durch Fragebogenitems für alle Messzeitpunkte vor Beginn der Trainingsreihe festgelegt. Dabei war oberste Prämisse die Evaluationsstandards Nützlichkeit, Durchführbarkeit, Genauigkeit und Fairness für alle Teilnehmenden und Betroffenen sicherzustellen. Daher wurde hier bewusst ein partizipativer Evaluationsprozess gewählt, bei dem frühzeitig und mehrfach das auftraggebende Projektteam sowie der Betriebsrat in die Planung des Evaluationsprozesses und die

${ }^{36}$ Für den deutschsprachigen Raum stellt die Gesellschaft für Evaluation (DeGEval, 2016) darauf basierende Standards und Checklisten zur Qualitätssicherung von Evaluationen zur Verfügung (https://www.degeval.org/degeval-standards/download/). 
Konzeption der Erhebungsinstrumente einbezogen wurde. Daraus ergab sich, neben Restriktionen bezüglich Erhebungsumfang und Anzahl an Messzeitpunkten, auch die Anforderung, dass die Skalen dafür passend konstruiert werden mussten und daher validierte Erhebungsinstrumente nur in modifizierter Form herangezogen werden konnten. Dies erhöhte die Wahrscheinlichkeit suboptimaler interner Konsistenz und damit einer Reduktion der statistischen Power bzw. einer Erhöhung der BetaFehlerwahrscheinlichkeit ${ }^{37}$. Maßnahmen zur Reduktion von Verzerrungen aufgrund einer Selbstevaluation bestanden in der Kontrolle von Ausgangswerten und Nutzung verschiedener Messzeitpunkte von Prädiktor und Kriterium sowie Empfehlungen zur Fragebogenkonstruktion (Podsakoff et al., 2003; Podsakoff et al., 2012), um Effekte von Antworttendenzen zu reduzieren (z. B. soziale Erwünschtheit oder Common-MethodBias). Durch das geschlossene Antwortformat basierend auf einer fünfstufigen LikertSkala wurde zudem der Interpretationsspielraum der Antworten minimiert und damit die Auswertungsobjektivität gefördert. Die Datenanalysen wurden zudem möglichst transparent beschrieben und basierend auf methodischen Standards bzw. gängigen Konventionen durchgeführt. Allerdings muss berücksichtigt werden, dass rein quantitative Verfahren mit einem "Informationsverlust", insbesondere bezüglich Hintergründe von Effekten im Vergleich zu qualitativen Verfahren, verbunden sind. Da hier jedoch eine Integration summativer und formativer Evaluation gewählt wurde, können auch aus dieser rein quantitativen Datenquelle gewisse Hintergründe erfasst werden. Zudem wurden im Rahmen freiwilliger individueller Rückmeldegespräche mit den Teilnehmenden der Transfererfolg und dessen Rahmenbedingungen in einem Beratungsformat mit der Trainerin bearbeitet. Dabei wurde basierend auf halbstrukturierten Leitfäden auch eine Bewertung des Trainings- und Evaluationsprozesses qualitativ abgefragt. Ergebnisse aus 12 Gesprächen zeigten, dass trotz Selbstevaluation und der hier selbstselektierten Stichprobe (von der angenommen werden kann, dass sie eine besonders hohe Lern- und Transfermotivation hat) auch offen Kritik, Verbesserungspotenziale und Einschränkungen des Trainingstransfers geäußert wurden. Diese Rückmeldungen bezogen sich relativ übereinstimmend auf drei zentrale Aspekte ${ }^{38}$ : 1) Trainingsbaustein 1 wurde als $z u$ theoretisch und damit relativ praxisfern erlebt, dagegen wurde die Behavior Modeling Methode der anderen Bausteine bei allen Gesprächen als sehr bereichernd und überraschend realistisch bewertet. 2) Große Herausforderung bei der Umsetzung der persönlichen Entwicklungsziele stellte der stressige und komplexe Arbeitsalltag dar, der zu wenig

\footnotetext{
${ }^{37}$ Wahrscheinlichkeit, dass ein vorhandener Effekt nicht identifiziert wird.

${ }^{38}$ Ausführlichere Analyseergebnisse der qualitativen Gesprächsdaten können bei der Autorin erfragt werden.
} 
Transfermöglichkeiten bietet. Zudem wünschten sich vier Teilnehmende die Möglichkeit, herausfordernde Gesprächssituationen, die in der Transferzeit aufgekommen sind durch weitere Rollenspiele mit Videofeedback zu üben, da nicht alle schwierigen Gesprächssituationen nach der Trainingsreihe zufriedenstellend gelöst werden konnten. 3) Hauptkritik an der Trainingsreihe waren die zu umfassenden Fragebögen, die einerseits als teilweise redundant aufgrund ähnlicher Fragen, andererseits als zu kompliziert (insbesondere durch invertierte und umständliche Fragen) erlebt wurden. Es wurde jedoch von einigen Teilnehmenden als positiv erlebt, dass durch die Fragebögen mehrfach an das Training vor- und nachbereitend erinnert wurde. Insbesondere die Befragung nach dem Training wurde als Lernerfolgskontrolle geschätzt, wobei sich mehrere Teilnehmende mehr Zeit zur Bearbeitung gewünscht hätten. Insgesamt wurde die prozessbegleitende Evaluation zumindest von den Teilnehmenden der individuellen Rückmeldegespräche als fair und nützlich erlebt, wobei die Freiwilligkeit der Evaluation positiv hervorgehoben wurde.

Neben der Selbstevaluation birgt auch die Wahl einer Einzelevaluation durch nichtexperimentelle Überprüfung einer Trainingsreihe ohne Vergleichsinterventionen methodische Nachteile. Auch wenn dies in der Trainingsforschung gängig ist, sind derartige Designs insbesondere für Kausalschlüsse bzw. interne Validität problematisch (siehe auch Martin et al., 2017). Die Effekte der ergebnisorientierten Wirksamkeitsüberprüfung könnten auch durch andere Ereignisse bzw. Entwicklungen im Unternehmen oder persönlicher Entwicklungsprozesse verursacht worden sein und nicht durch die Intervention. Dies ist umso wahrscheinlicher je größer der Abstand zwischen den betrachteten Messzeitpunkten ist. Da hier eine relativ lange Transferzeit betrachtet wurde, wurden die Eingruppen-Prä-Posttests durch einen Wartelistenkontrollgruppenvergleich ergänzt. Auch wenn Ergebnisse dieses Designs nicht so belastbar sind wie von experimentellen Designs mit Vergleichsinterventionen und die Post-Erhebung der Experimentalgruppe ca. 1 Jahr vor der Prä-Messung der Wartelistenkontrollgruppe stattfand, wurden die Ergebnisse des Eingruppen-Prä-Posttestplans weitestgehend bestätigt. Daher wird eine Rückführung der Effekte auf die Intervention als auf unbekannte Faktoren für plausibler erachtet. Andererseits kann angenommen werden, dass der Trainings- und Transfererfolg der früher Trainierten Spillovereffekte durch Modelllernen im Arbeitsalltag der später trainierten Führungskräfte hatte. Dennoch waren alle Kriterienausprägungen der bereits Trainierten größer als der noch Untrainierten, wobei die meisten Erfolgskriterien der Experimentalgruppe auch (marginal) signifikante Überlegenheit im Vergleich zur Wartelistenkontrollgruppe aufwiesen. Die Ergebnisse des Wartelistenkontrollgruppenvergleichs können somit eher als unterschätzt betrachtet werden. Allerdings muss berücksichtigt werden, dass sich die Vergleichsgruppen hoch signifikant bezüglich der Ausprägung von 
Hierarchiestufe und Alter sowie marginal signifikant bezüglich Führungserfahrung unterschieden. Daher sollten die Ergebnisse des Wartelistenkontrollgruppenvergleichs unter Betracht der Möglichkeit interpretiert werden, dass sie durch diese systematischen Unterschiede der Kontrollvariablen beeinflusst wurden. Aufgrund der Nachteile eines Kontrollgruppenplans insbesondere bezüglich Kontrolle der Teilnehmendenmerkmale gelten Within-Designs daher als belastbarer für Trainingsevaluationen (Frese et al., 2003; Hunter \& Schmidt, 1990). Dennoch muss berücksichtigt werden, dass ohne experimentelles Studiendesign Kausalannahmen nicht als bestätigt gelten können und daher nur aufgrund von Plausibilität als wahrscheinlich bzw. möglich betrachtet werden können.

\subsubsection{Evaluationsgegenstand}

Evaluationsgegenstand war eine spezifische Führungskräftetraining mit Transfermanagementmaßnahmen zur Vermittlung bewährter Führungsstrategien (transformationales, transaktionales und interaktionales Führungsverhalten) anhand relativ typischer Inhalte (z. B. erfolgreiche Kommunikation, Aufbau von erwünschtem Mitarbeitendenverhalten, Leistungsrückmeldungen). Diese wurde in einem repräsentativen deutschen, international tätigen Unternehmen der Maschinenbaubranche durchgeführt, das ca. 450 Mitarbeitende in Verwaltung und Produktion beschäftigte. Dabei wurden alle Mitarbeitende Führungskräfte des Unternehmens trainiert und in der freiwilligen Evaluation berücksichtigt.

Neben der Kontrolle von Teilnehmendeneigenschaften war dadurch auch die Kontrolle wesentlicher Konfundierungen durch Konstanthaltung von Trainings- und Organisationssetting möglich. Zudem konnten auch ergänzende Wartelistenkontrollgruppenvergleiche sinnvoll durchgeführt werden, was bei verschiedenen Unternehmenskontexten nur bedingt möglich gewesen wäre. Allerdings war dadurch die erreichbare Stichprobengröße relativ limitiert und die statistische Power sowie die externe Validität beschränkt. Eine geringe statistische Power führt dazu, dass nur sehr große Effekte auch signifikant werden. Daraus kann geschlossen werden, dass die signifikanten Effekte der Evaluationsergebnisse relativ bedeutsam sind. Allerdings wurde zugunsten eines möglichst umfassenden Erkenntnisgewinns trotz mehrerer Testungen an einem Datensatz auf eine Alphaadjustierung verzichtet. Daher könnte ein Teil der signifikanten Ergebnisse auch durch Zufall bedingt sein. Aufgrund der ganzheitlichen theoriegeleiteten Hypothesenformulierung und den relativ konsistenten und plausiblen Ergebnissen kann jedoch angenommen werden, dass die Wahrscheinlichkeit zufälliger Effekte sehr gering ist. Externe Validität kann dennoch nur aufgrund der konsistenten Bestätigung bzw. Ergänzung bisheriger Forschung mit anderen Stichproben bzw. Trainings- und Organisationssettings 
angenommen werden. Zudem muss beachtet werden, dass es sich bei den Teilnehmenden nicht um unabhängige Zufallsziehungen handelte. Aufgrund von organigrammbedingten Abhängigkeiten bezüglich Bereiche, sowie aufgrund der Trainingsgruppen handelt es sich in mehrfacher Hinsicht um genestete Datenstrukturen (K. S. Cortina \& Pant, 2009). Zugunsten der Praktikabilität bzw. Durchführbarkeit wurde jedoch wie in der Trainingsforschung üblich auch hier das methodische Ideal der Berücksichtigung der genesteten Datenstruktur durch hierarchische lineare Modelle vernachlässigt.

Ein zentrales Problem prozessorientierter Trainingsforschung besteht in komplexen Modellen zu deren Überprüfung nur begrenzte Stichproben in vorwiegend retrospektiven Teilüberprüfungen realisiert werden, während konfundierende Einflüsse und zeitliche Wirkzusammenhänge kaum berücksichtigt werden (siehe Abschnitt 3.2.2 ab Seite 70). Auch im vorliegenden Forschungsprojekt ist es nicht möglich ein komplettes Transfermodell inferenzstatistisch zu überprüfen. Aufgrund der Komplexität des Transferprozesses mit zahlreichen Transferdeterminanten wäre eine Stichprobe mit über 300 Probanden erforderlich (Moosbrugger \& Rauch, 2009), die entsprechend der Forschungsfragen aus Führungskräften bestehen sollte bei denen transferrelevante Einflussgrößen aus Arbeitsumfeld, Trainingsgestaltung und Teilnehmendenmerkmale kontrolliert bzw. konstant gehalten werden müssen. Dass dies in Feldstudien kaum zu realisieren ist, zeigt sich auch in dem Überblick der Evaluationsforschung von Führungskräfteentwicklungsmaßnahmen (siehe Abschnitt 3.1.3 ab Seite 41). Von 335 Evaluationsstudien, die in einer aktuellen Metaanalyse zu Führungskräfteentwicklung erfasst wurden (Lacerenza et al., 2017), gibt es zwischen 1951 und 2014 nur sieben Studien, die Stichproben über 300 Probanden aufweisen. $73 \%$ der erfassten Studien haben eine Stichprobe unter $N=$ 50. Führungskräftetrainingsevaluationen, die erforderliche Stichprobengrößen für Strukturgleichungsmodelle oder umfassende Mediationsanalysen erreichen, basieren auf reinen Nachbefragungen ohne Kontrollgruppenvergleich oder fassen Trainingseffekte unterschiedlicher Trainingsmaßnahmen bzw. unterschiedlicher organisationaler Settings zusammen (z. B. Alexander, 2014; Aragon \& Valle, 2013; Bramley, 1999; L. A. Burke \& Hutchins, 2008; Clark, 1982; Reid et al., 2003; Torp, 2008; Zohar, 2002). Dies gilt auch für aktuellere Studien (z. B. Mensmann \& Frese, 2019; Seiberling \& Kauffeld, 2017). Dabei werden jedoch konfundierende Effekte zentraler Einflussvariablen beispielweise durch erhebliche Unterschiede bezüglich Komplexität der Inhalte, Trainingssettings oder Arbeitskontexten kaum bis gar nicht kontrolliert (Blume et al., 2010; Holton et al., 2003; Lacerenza et al., 2017; Laker \& Powell, 2011). Eine Ausnahme stellt die Studie von Culpin, Eichenberg, Hayward und Abraham (2014) dar. Jedoch wurden dabei nur die Effekte eines fundierten Trainingsdesigns auf Transferintention und Transferleistung 
analysiert. Eine aktuelle Studie von Massenberg et al. (2017) stellt eine weitere Ausnahme dar $(\mathrm{N}=353$, Rücklaufquote $=65 \%)$, allerdings nur bezüglich der Erfassung von Transferfaktoren. Die Transferleistung haben jedoch nur 65 Teilnehmende eingeschätzt, weshalb gerade die entscheidende Variable aufgrund der geringen Rücklaufquote aus den Analysen ausgeklammert wurde. Auch Evaluationsstudien aus dem militärischen (z. B. Smy, Shelton, Tombs \& Patrick, 2016; Towler, Watson \& Surface, 2014) oder universitären Kontext (z. B. Al-Swidi \& Al Yahya, 2017; Medina, 2017; Paulsen \& Kauffeld, 2017) warten mit großen Stichprobengrößen auf. Allerdings besteht dabei das Problem, dass eine Übertragung der Ergebnisse auf den Wirtschaftskontext nicht ohne weitere Überprüfungen an Führungskräften von Unternehmen sinnvoll vorgenommen werden kann.

Daher wurde in diesem Forschungsprojekt zugunsten der Stichprobenpassung die realisierbare Stichprobengröße als akzeptabel erachtet. Dafür wurde möglichst umfassend versucht Probleme abzufangen, die kleinere Stichproben mit sich bringen (z. B. durch Mehrfachmessung, ergänzende Bootstrapping-Analysen oder Kontrolle von Teilnehmendeneigenschaften, Trainings- und Organisationssetting). Dennoch sollten die Ergebnisse und insbesondere die explorativen Annahmen an Stichproben mit weiteren Trainings- und Organisationssettings repliziert werden. Dabei sollten jedoch auch die damit einhergehenden konfundierenden Einflüsse sowie die Teilnehmendeneigenschaften (z. B. durch Kontrolle um Ausgangswerte der abhängigen Variablen) kontrolliert werden. Ansonsten sind die Vorteile größerer Stichproben aufgrund der Zunahme konfundierender Effekte nur bedingt gegeben.

\subsubsection{Operationalisierung}

Als zentrale Datenquelle der Evaluation wurden, wie in der Trainingsforschung gängig, Selbsteischätzungen per Fragebogen gewählt. Auch wenn Kennzahlen, Verhaltensbeobachtungen oder Einschätzung Dritter als häufig objektivere Datenquellen gelten, sind für die zu beantwortenden Forschungsfragen Selbsteinschätzungen die adäquateste Messmethode. Gerade bei individueller Transferleistung ist weniger entscheidend, ob Transferdeterminanten „objektiv“ transferförderlich ausgeprägt sind oder nicht, sondern inwiefern sie von den Teilnehmenden als transferförderlich wahrgenommen werden $(\mathrm{H}$. M. Hutchins, Nimon, Bates \& Holton, 2013; Massenberg \& Kauffeld, 2015; Podsakoff et al., 2012). Zudem gibt es einige zentrale Variablen, die per Definition innere Zustände erfassen (z. B. motivationale Aspekte), die nur durch Selbsteinschätzung erfasst werden können (Gegenfurtner et al., 2009). In der Trainingsforschung wurde entsprechend mehrfach bestätigt, dass die individuelle Wahrnehmung der Teilnehmenden nicht nur die entscheidendste Datenquelle ist, sondern auch im Vergleich mit objektiveren Kriterien 
valide ist (Chiaburu et al., 2010; Facteau et al., 1995; Podsakoff et al., 2012; Spector, 2006). Andererseits besteht bei objektiveren Erfolgskriterien eine größere Wahrscheinlichkeit von Asymmetrien zwischen Wirkkriterium und Maßnahme (Wittmann, 1990): Dritte bekommen in der Regel nur einen Teil der Situationen mit in denen Transferverhalten gezeigt wird, während die Teilnehmenden selbst alle Situationen berücksichtigen können und daher die aussagekräftigsten „Experten“ für die Einschätzung ihres Trainings- und Transfererfolgs sowie dessen Prädiktoren darstellen (Gessler, 2012).

Im Gegensatz zu gängiger Transferforschung mit reinen retrospektiven Trainings- und Transferevaluationen (Baldwin et al., 2017; Ford et al., 2018), wurden Transferdeterminanten sowie Trainings- und Transferkriterien in einem längsschnittlichen Design mit mehreren Messzeitpunkten erfasst. So konnten einerseits kurzfristige und langfristige Effekte zeitlich passend erfasst werden und die Effekte der relevanten Prädiktoren gemäß ihrer zeitlichen Wirkung präziser in den Verlauf des Trainings- und Transferprozesses eingeordnet werden. Allerdings weisen insbesondere die Ergebnisse zu Transfereffekten der Transfermotivation darauf hin, dass diese Transferdeterminante zusätzlich über die Transferzeit hinweg erfasst werden sollte: Einerseits damit ausreichend Möglichkeit besteht, entwickelte Kompetenzen und ihre Umsetzung zu reflektieren (siehe auch Massenberg \& Kauffeld, 2015). Andererseits weil ihre Ausprägung entscheidend von Konsequenzen erster Transferversuche gemäß eines dynamischen Interaktionismus zwischen Situation und Person beeinflusst wird (Blume et al., 2019). Zudem konnten durch Nutzung verschiedener Messzeitpunkte indirekte Veränderungsmessungen erfasst werden. Zwar muss berücksichtigt werden, dass auch bei Verwendung identischer Items diese zu unterschiedlichen Messzeitpunkten für die Teilnehmenden eine unterschiedliche Bedeutung haben können (Martin et al., 2017). Dennoch war dadurch eine relativ gute Kontrolle der Ausgangswerte und Reduktion konfundierender Teilnehmendeneigenschaften möglich, die gerade bei subjektiver Selbsteinschätzung vielfältige Verzerrungen (z. B. soziale Erwünschtheit, Common-Method-Bias) bedingen (Podsakoff, MacKenzie, \& Podsakoff, 2012; Scriven, 1991). Aufgrund der Mehrfachmessungen sind zudem Test- und Erinnerungseffekte insbesondere bei der ergebnisorientierten Wirksamkeitsprüfung von Transfermotivation, Kompetenzentwicklung und Transferverhalten wahrscheinlich (siehe auch Martin et al., 2017). Da dies jedoch im Rahmen der Transferförderung durch Evaluation intendiert war, wird dies hier nicht als Verzerrung oder Limitation der Ergebnisse betrachtet. Zudem bestätigten auch die Erfolgskriterien ohne Mehrfachmessung den Trainings- und Transfererfolg. Schließlich bringen Studiendesigns mit Mehrfachmessung bzw. indirekte Veränderungsmessungen mögliche Verzerrungen aufgrund von Deckeneffekten oder Regression zur Mitte mit sich (Martin et al., 2017). Dies könnte beispielsweise ein Grund für den nicht signifikanten Zuwachs 
der interaktionalen Führung sein, die bereits einen Ausgangswert von 4 auf der fünfstufigen Skala hatte.

Allerdings bringen Mehrfachmessungen bei kleinen Stichproben verstärkt Probleme unvollständiger Rücklaufquoten bzw. Mortalität mit sich. Dies könnte die Ergebnisse verzerrt haben, da angenommen werden kann, dass es einen Zusammenhang zwischen Rücklauf und Prädiktor- sowie Kriteriumsvariablen gibt (z. B. Teilnehmende die hohe Transfermotivation und -erfolge haben melden dies eher zurück als Teilnehmende mit geringer Ausprägung, siehe auch Abschnitt 5.3.3 ab Seite 110). Somit waren imputationsbasierte Schätzungen zur statistischen Vervollständigung der Datensätze nicht sinnvoll nutzbar. Entsprechend musste ein Vorgehen mit unvollständigen Datensätzen gewählt werden. Um möglichst wenig Fälle ausschließen zu müssen, wurde in den jeweiligen Analysen ein listenweiser Fallausschluss gewählt. Entsprechend dem Prinzip des paarweisen Fallausschlusses wurde für jede Analyse die maximal verfügbare Probandenzahl verwendet, die für alle dabei analysierten Variablen mindestens einen Wert angegeben hatten. Zudem wurden Regressionsanalysen, deren Robustheit und Belastbarkeit stärker von der Stichprobengröße abhängt als bei t-Tests, zusätzlich mit Bootstrapping-Analysen und Mittelwertsubstitution durchgeführt, um die Ergebnisse an einer Teststärkeren Analyse zu überprüfen. Diese Zusatzanalysen bestätigten die gefundenen Effekte weitestgehend. Durch diese Vorgehensweisen konnte die Größe von Analysestichproben und somit Teststärken unter den gegebenen Rahmenbedingungen für die jeweiligen Analyseverfahren maximiert werden. Allerdings muss berücksichtigt werden, dass die einzelnen Analysen damit auf verschiedenen Teilstichproben basieren. Ganzheitliche Mediationsanalysen waren somit nicht sinnvoll durchführbar, da sich die fehlenden Werte der zu betrachtenden Messzeitpunkte kumulieren. Zudem darf nicht übersehen werden, dass das Problem möglicher Verzerrungen aufgrund eines Zusammenhangs zwischen Rücklauf und Prädiktor- sowie Kriteriumsvariablen nicht gelöst ist, da in die Analysen trotzdem nur die Teilstichproben eingehen, die zu den jeweilig betrachteten Messzeitpunkten einen Fragebogen abgegeben bzw. mindestens ein Item der betrachteten Variablen angegeben haben. Da die Rücklaufquoten aller Messzeitpunkte jedoch im Vergleich zu anderen Evaluationsstudien als gut bis sehr gut betrachtet werden können, sind rücklaufbedingte Verzerrungen der identifizierten Effekte aufgrund von Abdeckungs- und Nonresponsefehlern in der vorliegenden Studie als vergleichsweise gering zu erachten (siehe auch Modell des Total Survey Error; Groves et al., 2002; Groves et al., 2009). 
Die Skalenbildung der Transferdeterminanten basierte auf dem Modell des GLTSI, das entsprechend des aktuellen Forschungsstandes (siehe Abschnitt 3.2.1 ab Seite 45) um weitere betätigte Transferdeterminanten ergänzt und auf den Evaluationsgegenstand angepasst wurde. Die Operationalisierung der Trainings- und Transfererfolgskriterien folgte dem multidimensionalen Ansatz von Kirkpatrick $(1967,1998)$. Auf der Ebene des Transferverhaltens wurden zudem Empfehlungen der Transferforschung aufgegriffen indem auch hier präzise und multidimensional naher und weiter Transfer differenziert wurde und dafür ein angemessener sowie präzisierter zeitlicher Abstand zum Training gewählt wurde (Arthur et al., 2003; Ford et al., 2018; Laker, 1990). Skalen des nahen Transfers basierten auf den unmittelbaren Trainingsinhalten sowie modifizierten Items eines validierten Messinstruments, der Self-Rated Emotional Intelligence Scale nach Brackett et al. (2006). Inhaltlich besaßen sie eine Schnittmenge mit den Kompetenzskalen der jeweiligen Trainingsbausteine. Skalen des fernen Transfers basierten auf modifizierten Items deutscher Versionen des Multifactor Leadership Questionnaire (MLQ 5x; Bass \& Avolio, 1995) sowie der Leader-Member-Exchange-7-Item-Skala (LMX7; Graen \& Uhl-Bien, 1995). Bezüglich der Erhebungszeitpunkte muss berücksichtigt werden, dass mit dem zeitlichen Abstand zwischen den Messungen bzw. zum Training auch die Wahrscheinlichkeit konfundierender Ereignisse zunimmt. Andererseits kann gerade bei fernem Transfer eine relativ lange Zeit für eine erfolgreiche Etablierung von Transferverhalten erforderlich sein. Der relativ groß gewählte Abstand im Vergleich zu gängiger Trainingsforschung (über 6 Monate nach dem Training), könnte insbesondere für die interaktionale Führung aufgrund ihrer Abhängigkeit von einer aktiven individuellen Beziehungsgestaltung mit allen Mitarbeitenden noch zu kurz für eine Erfolgsüberprüfung gewesen sein. Dies könnte (neben einem Deckeneffekt) ein Grund für den nicht signifikanten Zuwachs dieses Transferkriteriums auf der Makroebene sein. Bei der Erklärung individueller Transferleistung zeigten sich jedoch die angenommenen Zusammenhänge mit zwei der drei primären Transferdeterminanten. Die Operationalisierung mittels selbstkonstruierter Skalen ${ }^{39}$ war insbesondere aufgrund der Prämissen Fairness, Nützlichkeit und Durchführbarkeit erforderlich, um Trainingsinhalt, Organisationssetting und lizenzrechtliche Rahmenbedingungen angemessen zu berücksichtigten. Dies brachte jedoch gewisse Einbußen bezüglich Genauigkeit mit sich. Einerseits erhöht der Einsatz nicht-validierter Messinstrumente Probleme der Konstruktvalidität. Somit sind auch die Ergebnisse bezüglich etablierter Konstrukte (transformationale, transaktionale und interaktionale Führung sowie LTSI-Skalen) nur bedingt mit anderen Studien vergleichbar.

39 Dies war unter anderem aufgrund des partizipativen Evaluationsansatzes erforderlich, bei der die Evaluationskriterien und Erhebungsverfahren von den Auftraggebenden (die auch Teilnehmende waren) genehmigt bzw. auch angepasst wurden. 
Andererseits sind bei nicht-validierten Messinstrumenten Verzerrungen aufgrund von suboptimaler interner Konsistenz insbesondere bei Mehrfachmessungen sehr wahrscheinlich und schwächen die statistische Power. In den vorliegenden Datenanalysen zeigte sich dies bei den Transferskalen naher Transfer von TB3 und transaktionaler Führung sowie bei den Subskalen der Transferdeterminanten Kollegenunterstützung und Feedback zu T0 als auch bei Transferdesign und Anwendungsmöglichkeit zu T2. Dadurch könnten mögliche Effekte unterschätzt bzw. nicht identifiziert worden sein.

Insgesamt zeigt die reflektierte Metaevaluation auf, dass die Evaluationsstandards im Evaluationsprozess möglichst umfassend durch verschiedene Ansätze umgesetzt wurden. Durch die Wahl des Evaluationsdesigns mittels Selbst- und Einzelevaluation, das bereits frühzeitig einen partizipativen Ansatz durch Einbezug von Teilnehmenden und Betroffenen in die Planung realisierte, wurden die Evaluationsstandards Durchführbarkeit, Fairness und Nützlichkeit verfolgt. Auf Genauigkeit wurde insbesondere durch die multidimensionale Operationalisierung mit mehreren Messzeitpunkten und Nutzung wissenschaftlicher Standards bei den darauf basierenden Datenanalysen geachtet. Optimierungen für weitere Evaluationen ergeben sich insbesondere aufgrund der dargestellten Limitationen der Evaluationsergebnisse bezüglich interner und externer Validität sowie der statistischen Power. Daher sollten die identifizierten Wirkzusammenhänge an Stichproben in weiteren kontrollierten Trainings- und Organisationssettings überprüft werden. Dabei sollten Stichprobengrößen generiert werden, die eine ganzheitliche Modellprüfung beispielsweise mittels Strukturgleichungsmodellen zulassen. Zudem sollten im Sinne der Evaluationsstandards der Einsatz von Fragebögen insbesondere bezüglich des Umfangs und vereinfachter Formulierung optimiert werden. Dabei wird, soweit möglich, Nutzung validierter Messinstrumente empfohlen. Allerdings sollte die oberste Prämisse im Sinne der Durchführbarkeit und Nützlichkeit eine Kriterienpassung zum Trainingsinhalt, Organisationssetting und Evaluationsziel sein. Dies könnte sich neben wahrgenommener Nützlichkeit und Fairness sowie vereinfachter Durchführbarkeit auch positiv auf die Rücklaufqouten und die Genauigkeit auswirken. Zudem könnte eine zusätzliche Erfassung der Transferdeterminanten über die Transferzeit hinweg weiteren Erkenntnisgewinn liefern. 


\subsection{Kapitelzusammenfassung}

Die ergebnisorientierte Wirksamkeitsprüfung zeigte, dass Forschungsbefunde und deren Empfehlungen für transferförderliche Trainingsgestaltung in Trainings zur Vermittlung typischer Führungskompetenzen ${ }^{40}$ wirksam und praktikabel angewendet werden können. Die Überprüfung der Hypothese 1 bestätigte relativ konsistent die Trainingswirksamkeit (a) bezüglich kurzfristigem Trainingserfolg durch positive Trainingsreaktion (Nützlichkeitseinschätzung und Transfermotivation) und Kompetenzentwicklung sowie (b) bezüglich langfristigem Transfererfolg durch Transferverhalten (naher und weiter Transfer) und dessen Resultate (Verbesserung in Führungskommunikation und -verhalten). Nur für Transfermotivation TB1 als ein zusätzlicher Indikator affektiver Trainingsreaktion (möglicherweise, weil es sich um eine relativ kurze Einführungsveranstaltung mit Fokus auf Wissensvermittlung handelte) und interaktionale Führung (möglicherweise aufgrund eines Deckeneffekts oder zu geringer Transferzeit) wurde der Zuwachs nicht signifikant. Die Ergebnisse wurden durch ergänzende Wartelistenkontrollgruppenvergleiche und Mitarbeitendeneinschätzungen der Resultatebene gestützt. Damit kann der Trainings- und Transfererfolg auf gesamtorganisationaler Makroebene als bestätigt gelten, der sich nicht nur unmittelbar nach dem Training in Reaktion und Kompetenzzuwachs zeigt, sondern auch noch ca. sieben Monate nach der Trainingsreihe im Transferverhalten und dessen Resultaten.

Durch die prozessorientierte Wirksamkeitsprüfung wurden dahinterliegende Wirkmechanismen auf individueller Mikroebene ergründet, um die Forschungsfragen zu klären wie die Wirkmechanismen des Trainingstransfers bei Führungskräftetrainings zusammenhängen, welche Transferdeterminanten für individuelle Transferleistung von besonderer Bedeutung sind und ob diese durch transferförderliche Trainingsgestaltung vorhergesagt werden können.

Dafür wurden zunächst die Transfereffekte durch direkte Prädiktoren regressionsanalytisch überprüft. Hypothese 2 wurde allerdings nur teilweise bestätigt: Transfer konnte relativ konsistent und maßgeblich durch (a) Kompetenz und (c) Transfermöglichkeit vorhergesagt werden, (d) wobei Kompetenz den größten Einfluss der angenommenen primären Transferdeterminanten zeigte. Eine Ausnahme stellte das Transferkriterium transaktionale Führung dar, bei der nur Hypothese 2 (c) bestätigt werden konnte. Allerdings könnte dies auch durch die suboptimale interne Konsistenz dieser Skala bedingt

40 Training bewährter Führungsstrategien (transformationales, transaktionales und interaktionales Führungsverhalten) anhand relativ typischer Inhalte (z. B. erfolgreiche Kommunikation, Aufbau von erwünschtem Mitarbeitendenverhalten, Leistungsrückmeldungen). 
sein. Zudem konnten Transfereffekte der Transfermotivation nur bedingt durch marginal signifikante Transfereffekte bei nahem Transfer und transformationaler Führung bestätigt werden. Daher konnte Hypothese 2 (b) durch die Datenanalysen nicht klar bestätigt werden. Aufgrund der zahlreichen Studien, die die zentrale Bedeutung der Transfermotivation als direkten Prädiktor von Transfer nachgewiesen haben (siehe Abschnitt 3.2.1 ab Seite 45), werden die hier inkonsistenten Ergebnisse eher auf methodische Aspekte wie beispielsweise suboptimale interne Konsistenz der transaktionalen Führung, Erhebungszeitpunkte von Prädiktor und Kriterium (im Falle der interaktionalen Führung) und geringe statistische Power aufgrund der Stichprobengröße zurück geführt. Explorative Ergebnisse weisen zudem darauf hin, dass die übrigen Transferdeterminanten Kriterien übergreifend nur eine untergeordnete bzw. sekundäre Rolle spielen. Einzige Ausnahme stellen die Skalen Sanktionen (invers) und Trainingsdesign (in etwas geringerer Weise) dar. Weiterer Analysen legen jedoch auch hier vorwiegend indirekte Transfereffekte nahe.

In einem zweiten Schritt wurden sekundäre Determinanten zur Erklärung der primären Transferdeterminanten Kompetenz, Transfermotivation und -möglichkeit analysiert. Hypothese 3 (a) bezüglich der Vorhersagekraft sekundärer Teilnehmendeneigenschaften konnte für keine primäre Transferdeterminante bestätigt werden. Allerdings muss berücksichtigt werden, dass die LTSI-Skalen für diese Studie selbstkonstruiert wurden und nicht die validierten Items verwendet werden konnten. Der Einfluss von Arbeitsumgebung gemäß Hypothese 3 (b) wurde nur für Transfermöglichkeit gestützt. Hypothese 3 (c) und (d) bezüglich des Einflusses transferförderlicher Trainingsgestaltung konnte jedoch für alle drei primären Transferdeterminanten bestätigt werden. Explorative Analysen auf Subskalenniveau weisen darauf hin, dass spezifische Skalen transferförderlicher Teilnehmendenmerkmale, Arbeitsumgebung und insbesondere Trainingsgestaltung die primären Transferdeterminanten vorhersagen können. Zentrale Transfermechanismen wirken bereits vor und während Trainings auf Kompetenzentwicklung, Transfermotivation sowie erwartete Anwendungsmöglichkeit, während nach Trainings die tatsächliche Transfermöglichkeit sowie die Sicherung von Kompetenz und Motivation ihrer Anwendung von besonderer Bedeutung zu sein scheinen.

Trotz der diskutierten Limitationen, die in der Metaevaluation zusammengefasst wurden, liefert die vorliegende Forschungsarbeit damit einen Erkenntnisgewinn zur Erklärung von Trainings- und Transfererfolg auf organisationaler Ebene sowie der dahinterliegenden Wirkmechanismen auf individueller Ebene. Dabei wurden zentrale Forschungslücken aufgegriffen (z. B. ganzheitliche Erfassung von Transferdeterminanten und Erfolgskriterien, strikte Differenzierung von primären und sekundären Transferde- 
terminanten zur Erhöhung der Praxisorientierung und Präzisierung von Handlungsempfehlungen, Kombination von Eingruppen-Prä-Posttest und Wartelistenkontrollgruppenvergleich, Mehrfachmessungen über den Trainings- und Transferprozess hinweg, Kontrolle von Teilnehmendeneigenschaften, Trainings- und Organisationssetting, ausführliche Dokumentation der Intervention etc.). Insgesamt lieferten die umfassenden Datenanalysen nicht nur eine weitgehende Bestätigung des Studienmodells, sondern zusätzlich auch Anhaltspunkte für ein gezieltes Transfermanagement. Diese sollten durch weitere Forschung überprüft werden, wobei insbesondere statistische Power sowie interne und externe Validität der Ergebnisse optimiert werden sollten. 


\section{Leitfaden für systematisches Transfermanagement}

In diesem Kapitel werden Handlungsimplikationen für die Praxis in einem Leitfaden für ein systematisches Transfermanagement vorgestellt. Diese wurden aus theoriegeleitetem Erkenntnisgewinn und theoriebildenden Konsequenzen der Datenanalysen aus Kapitel 6 ab Seite 139 abgeleitet. Dafür werden die Ergebnisse der Hypothesentestungen und explorative Analysen zur Wirksamkeitsprüfung eines Trainings zur Vermittlung zentraler Führungskompetenzen (transformationales, transaktionales und interaktionales Führungsverhalten), vorbehaltlich der diskutierten Limitationen (siehe Abschnitt $6.4 \mathrm{ab}$ Seite 171), integriert. Dadurch werden vorliegende theoriegeleitete Handlungsempfehlungen zu übergeordneten Transfermanagementansätzen (z. B. L. A. Burke, Hutchins \& Saks, 2013; Felfe \& Franke, 2014; Salas et al., 2012; Solga, 2011b), durch aktuelle Forschung und ganzheitliche empirische Fundierung ergänzt, konkretisiert und priorisiert.

Die übergeordnete Struktur orientiert sich an Transfermanagementmaßnahmen zur Förderung der primären Transferdeterminanten Kompetenz, Transfermotivation und -möglichkeit. Als zentrale direkte Prädiktoren von Kompetenz nach dem Training wurden das Kompetenzniveau vor dem Training, das Transferdesign während des Trainings sowie die Transfermotivation nach dem Training identifiziert. Als zentrale direkte Prädiktoren von Transfermotivation nach dem Training wurden die Transfermotivation vor dem Training, die durch das Training geförderte Anwendungsmöglichkeitserwartung sowie das Kompetenzniveau nach dem Training identifiziert. Als zentrale direkte Prädiktoren von Transfermöglichkeit in der Transferzeit wurden schließlich die Kollegenunterstützung ${ }^{41}$ vor dem Training sowie die durch das Training geförderte Anwendungsmöglichkeitserwartung identifiziert (siehe Abbildung 21 auf Seite 169). Wie diese Wirkmechanismen durch Maßnahmen vor, während und nach dem Training aus den in Abschnitt 5.2.2 ab Seite 95 beschriebenen Checklisten von Salas et al. (2012) gezielt unterstützt werden können ist nachfolgend zusammengefasst.

\footnotetext{
${ }^{41}$ Transferunterstützung durch Mitarbeitende und gleichgestellte Kollegen.
} 


\subsection{Transfermanagementmaßnahmen vor Trainings}

Als zentraler Ansatzpunkt des Transfermanagements zur Förderung von Kompetenzentwicklung wurde das Kompetenzniveau vor dem Training identifiziert. Aus der Checkliste für Maßnahmen vor Trainings von Salas et al. (2012) Abbildung 15 auf Seite 96 sollten daher insbesondere Aspekte umgesetzt werden, die das Vorwissen bezüglich zu trainierender Kompetenzen aktivieren. Im Kontext dieser Studie hat sich dafür eine Pre-Trainingintervention mit drei Schwerpunkten bewährt (siehe Abschnitt 5.2.2 ab Seite 95): 1) Vorinformation der Teilnehmenden bezüglich Relevanz, Inhalt und Ablauf; 2) Vorbefragungen bezüglich des Kompetenzniveaus und relevanter Transferdeterminanten im Rahmen der prozessbegleitenden Evaluation und 3) Einführungsveranstaltung mit dem Fokus auf Vermittlung von Basiswissen und Transferrelevanz einige Wochen vor dem eigentlichen Training (Trainingsbaustein 1). Diese Maßnahmen dienen auch der Förderung der Transfermotivation, die einerseits mit der Kompetenzentwicklung in Zusammenhang steht und andererseits als weitere primäre Transferdeterminante gilt. Da diese maßgeblich von der Transfermotivation vor dem Training vorhergesagt werden kann, sollten Wirkmechanismen unterstützt werden, die bereits das Ausgangsniveau begünstigen. Einerseits kann hier an den Teilnehmendenmerkmalen Lern- und Trainingsmotivation sowie der Erwartungsklarheit angesetzt werden. Auch dafür bewährte sich die bereits erwähnte Pre-Trainingintervention mittels Vorinformation, Vorbefragungen und Einführungsveranstaltung. Ergänzend wurde auch die Förderung von Trainings- und Transfermotivation durch Werbung für die Trainingsreihe über unternehmensinterne „Promotoren“ auf zentralen Plattformen (Betriebsversammlung und Intranet) positiv aufgenommen. Andererseits zeigten auch die Arbeitsumgebungsfaktoren erwartete Vorgesetzten- und Kollegenunterstützung (letztere insbesondere vermittelt über wahrgenommene Transfermöglichkeit in der Transferzeit) sowie Transferkonsequenzen und -kapazität transferförderliche Effekte. Bereits vor dem Training wurden Maßnahmen zur Förderung dieser zentraler Arbeitsumgebungsfaktoren hier ebenfalls durch die Pre-Trainingintervention über die Förderung der Teilnehmendeneinstellungen bezüglich Trainingsteilnahme und Transfer abgedeckt. Ergänzend könnten Maßnahmen zur Vorbereitung bzw. Einbezug der Vorgesetzten der Teilnehmenden eingesetzt werden ${ }^{42}$. In der vorliegenden Studie waren alle Führungskräfte auch Teilnehmenden, weshalb die Thematisierung von Transferrelevanz und -determinanten im Rahmen der Pre-Trainingintervention diesbezüglich als ausreichend erachtet wurde.

42 Z. B. Beteiligung an der Bedarfsanalyse, Aufforderung und Anleitung zur Durchführung von Entwicklungsgesprächen, Aufzeigen der Trainingsrelevanz oder Optimierung von Transferfaktoren der Arbeitsumgebung (siehe auch Kauffeld et al., 2009; Kauffeld et al., 2012). 
Zudem sollten bereits vor dem Training Maßnahmen zur Förderung des Transferdesigns ergriffen werden, um förderliche Gestaltungsaspekte der Trainingsdurchführung bezüglich effektiver Vermittlung der Trainingsinhalte, Methodenmix, Übungsmöglichkeiten sowie Kompetenz des Trainerteams (siehe Abschnitt 5.3.5 ab Seite 115) vorzubereiten. Denn diese Aspekte wirken einerseits direkt auf die Kompetenzentwicklung. Andererseits weisen die Analysen auch auf einen zusätzlichen indirekten Wirkmechanismus hin: Zusammen mit Inhaltsrelevanz (i. S. von realitätsnahen Inhalten, anwendungsorientierten Trainingsunterlagen und praxisnahen Fallbeispielen, siehe Tabelle 6 ab Seite 118) und Arbeitsumgebungsfaktoren (Vorgesetztenunterstützung, erwartete Transferkonsequenzen und -kapazität) fördert das Transferdesign die Anwendungsmöglichkeitserwartung. Diese steigert wiederum (zusammen mit Transfermotivation vor und Kompetenz nach dem Training) die Transfermotivation sowie die Transfermöglichkeit in der Transferzeit (siehe Abbildung 21 auf Seite 169 sowie Tabelle 17 auf Seite 167 und Tabelle 19 auf Seite 167).

Um das Training im Sinne des Transferdesigns auf die tatsächlichen Arbeitsanforderungen ausrichten zu können, ist aus der Checkliste für Maßnahmen vor Trainings von Salas et al. (2012) die Bedarfsanalyse bzw. insbesondere deren Aufgabenanalyse zur Spezifizierung der Kompetenz- und Arbeitsanforderungen wichtig. In der vorliegenden Studie wurden dafür Bewertungen zur Mitarbeiterführung und Lernkultur aus Interviews mit einer repräsentativen Stichprobe der Belegschaft aller Hierarchieebenen sowie der aktuellen schriftlichen Mitarbeiterbefragung genutzt (siehe Abschnitt 5.2.2 ab Seite ab Seite 95). Diese Analyseergebnisse dienten bei der Trainingskonzeption basierend auf transferrelevanten Lerntheorien ${ }^{43}$ auch zur Sicherung der Inhaltsrelevanz.

43 Z. B. Identische Elemente nach Thorndike und Woodworth (1901), Übertragung von Prinzipien nach Judd (1908) und situiertes Lernen nach Lave und Wenger (1991) bzw. Mandl et al. (2002). 


\subsection{Transfermanagementmaßnahmen während Trainings}

Zur Förderung der primären Transferdeterminanten Kompetenzentwicklung, Transfermotivation und -möglichkeit durch Maßnahmen während des Trainings wurde das Transferdesign als zentraler Prädiktor bestätigt. Zudem sollten auch die Anwendungsmöglichkeitserwartung und ihre antezedente Determinante Inhaltsrelevanz im Training gezielt gefördert werden.

Aus der Checkliste für Maßnahmen während Trainings von Salas et al. (2012) bewährte sich zur Förderung des Transferdesigns insbesondere der Einsatz einer effektiven und fundierten Trainingsstrategie mit einer auf die Trainingsziele abgestimmten Kombination aus Präsentationen, Diskussionen, Murmel- bzw. Kleingruppenarbeit sowie individueller Selbstreflexion mit einem Schwerpunkt auf Behavior Modeling Sequenzen (mit positiven und negativen Beispielen und Videofeedback, siehe auch Abschnitt $2.1 \mathrm{ab}$ Seite 7). Die Trainingsstrategie folgte den transferrelevanten Lerntheorien und integrierte selbstgesteuertes Lernen (Cappetta \& Paolino, 2015; Lourenco \& Ferreira, 2019; Sua Wui et al., 2018), Error-Management- und Teambased-Ansätze (Hart et al., 2019; Taylor et al., 2005). Diese Ansätze förderten im Sinne situierten Lernens offene Lernaktivitäten, Exploration und Austausch in konkreten Problemsituationen in einer kontextbezogenen sozialen Lernumgebung. Der erste Trainingsbaustein, der kein Behavior Modeling nutzte, da der Fokus auf Wissensvermittlung lag, wurde im Vergleich zu den anderen beiden Bausteinen weniger transferförderlich wahrgenommen ${ }^{44}$. Zudem wurde auch auf Inhaltsrelevanz durch hohe Anwendungs- und Transferorientierung (z. B. durch spezifische Praxisbeispiele in Präsentationen, Diskussionen und Rollenspielen, Reflexion von Transferhindernissen und deren Lösungsmöglichkeiten) geachtet. Dies wurde mittels Förderung der Lernorientierung durch Einforderung aktiver Teilnahme und Eigenverantwortlichkeit der Teilnehmenden für den Trainings- und Transferfolg und damit durch die explizite Übertragung von Transferverantwortung verstärkt.

Diese Maßnahmen sollten auch der Förderung von Anwendungsmöglichkeitserwartung dienen. Zudem bewährte sich aus der Checkliste für Maßnahmen während Trainings von Salas et al. (2012) die Verstärkung von Selbstwirksamkeit durch Ermutigung und Ermöglichung von Leistungserfolg. Dies erfolgte durch 1) kontinuierliches prozessbegleitendes Aufzeigen bisheriger Entwicklungsschritte (z. B. Rollenspiele mit ressourcenorientierten Feedback oder Reflexionsrunden und Zusammenfassung der neuen Erkenntnisse und deren Relevanz für individuelle Entwicklungsziele nach jedem über-

44 Siehe ergebnisorientierte Wirksamkeitsüberprüfung auf Reaktionsebene sowie mündliche Rückmeldungen. 
geordneten Inhaltsblock), 2) Lernerfolgskontrollen (z. B. durch Quizzrunden, Teilnehmendenpräsentationen zur Wiederholung von Inhalten, Evaluationsbögen unmittelbar nach den Trainingsbausteinen) sowie 3) Anwendungs- und Übungsgelegenheiten während und zwischen den Trainingsbausteinen mit ressourcenorientiertem Feedback (durch Trainerteam und andere Teilnehmenden im Rahmen angeleiteter Feedbackrunden zur Sicherstellung konstruktiven Feedbacks). Eine prozessbegleitende Evaluation kann die zentralen Wirkmechanismen auch während Trainings durch Anregung zur Reflexion von Kompetenzentwicklung und zentralen Transferdeterminanten unterstützen. 


\subsection{Transfermanagementmaßnahmen nach Trainings}

Auch nach dem Training sind Maßnahmen zur Förderung der primären Transferdeterminanten wichtig. Für Kompetenzentwicklung scheint insbesondere die Wiederholung der Inhalte relevant zu sein. Zur Förderung von Transfermotivation und -möglichkeit scheint insbesondere die wahrgenommene Anwendungsmöglichkeitserwartung zentral zu sein und sollte gemäß Zielsetzungstheorie (Locke \& Latham, 1990), der Theorie intentionaler Handlungssteuerung (Gollwitzer, 1993, 1999) bzw. Ansätzen zur Rückfallprävention bzgl. gewohnter Verhaltensweisen unterstützt werden ${ }^{45}$.

Aus der Checkliste für Maßnahmen nach Trainings von Salas et al. (2012) bewährte sich insbesondere für Kompetenzentwicklung und Transfermotivation grundsätzlich die prozessbegleitende Evaluation durch Fragebögen zur Selbstreflexion von Trainingsund Transfererfolg sowie dessen Prädiktoren. Diese wurde nicht nur vor, sondern auch unmittelbar nach den Trainings sowie nach einer längeren Transferzeit durchgeführt. Allerdings zeigten die individuellen Rückmeldegespräche, dass die wiederholten Reflexionsgelegenheiten zwar positiv aufgefasst wurden; eine weitere Optimierung bezüglich des Umfangs und einfacherer Formulierungen erscheint jedoch wichtig. Dafür sollten die Erhebungsinstrumente noch stärker als in den hier verwendeten Fragebögen an den Nutzenvorstellungen der Beteiligten ausgerichtet werden (z. B. gemäß utilizationfocused evaluation nach Patton, 1997). Dafür wird eine Orientierung an dem GLTSIModell $^{46}$ (siehe Abbildung 10 auf Seite 74) mit einer Priorisierung der hier als zentral identifizierten Transferdeterminanten (siehe Abbildung 21 auf Seite 169) und Fokussierung auf Kompetenzentwicklung empfohlen. Zudem sind die Handlungsempfehlungen zum Einsatz von Single-Item Measures nach Fisher et al. (2016) hilfreich, um den Umfang erforderlicher Fragen nach dem Sparsamkeitsprinzip zu reduzieren. Für die Trainings- und Transfererfolgskriterien kann soweit möglich die Orientierung an validierten Erhebungsinstrumenten empfohlen werden.

Aus der Checkliste für Maßnahmen nach Trainings von Salas et al. (2012) bewährten sich zudem insbesondere für die Förderung realisierter Transfermöglichkeit verschiedene Post-Traininginterventionen. Insbesondere die Förderung von Selbstmanagementkompetenzen bezüglich Transferverhaltens und dessen Aufrechterhaltung durch Abschlussreflexionen von Anwendungsmöglichkeitserwartungen und Beseitigung potenzieller Transferhindernisse unmittelbar nach dem Training auf Basis SMARTer Zielkarten (siehe Abbildung 19 auf Seite 107) wurde positiv aufgenommen. Gleichzeitig

\footnotetext{
45 Einen aktuellen Überblick über den Forschungsstand zu Post-Traininginterventionen geben Rahyuda et al. (2018).

46 Ergänzt um Lern- und Trainingsmotivation.
} 
sollten die persönlichen Zielkarten zusammen mit Trainingsunterlagen zur Autodidaktik und eigenen Nachverfolgung als zentrale Instrumente, Hilfsmittel und Handlungsempfehlungen zur Transferförderung nach dem Training dienen. Diese Materialien wurden von einigen Teilnehmenden auch noch ca. 1 Jahr nach der Trainingsreihe für die persönliche Entwicklungsreflexion genutzt. Eine Optimierung könnte in der alternativen Formulierung von intentionalen Handlungsabsichten (Gollwitzer, 1993, 1999) statt SMARTer Ziele sein, da sie noch stärker auf eine Förderung der Transfermotivation ausgerichtet sind und für Teilnehmende häufig einfacher zu definieren sind. Weitere PostTraininginterventionen bestanden in Auffrischungssitzungen jeweils zu Beginn des nachfolgenden Trainingsbausteins sowie der Definition sogenannter Austauschpartner, mit denen die Teilnehmenden ihre Entwicklungsziele anhand der SMARTen Zielkarten reflektieren sollten. Zudem wurde ein freiwilliges Rückmeldegespräch ca. 1 Jahr nach dem Training angeboten, das von ca. einem Drittel der Teilnehmenden genutzt wurde. Während die Auffrischungssitzungen durch sehr aktive Teilnahme sehr gut angenommen und genutzt wurden, wurde die eigenverantwortliche Reflexion mit den definierten Austauschpartnern laut individueller Rückmeldungen trotz Anleitung und Erinnerung kaum genutzt. Entsprechend scheint es empfehlenswert Maßnahmen zur Anregung zu Reflexion und Erfahrungsaustausch bezüglich Umsetzung von Trainingsinhalten im Arbeitsalltag vor allem im angeleiteten Format durch Auffrischungssitzungen und individuelle Rückmeldegespräche umzusetzen. 


\subsection{Kapitelzusammenfassung}

Für theorie- und empiriegeleitete Handlungsempfehlungen eines systematischen und praxisorientierten Transfermanagements wurden in diesem Kapitel Wirkmechanismen und Transfermanagementmaßnahmen mit konkreten Umsetzungsbeispielen von der durchgeführten Trainingsevaluation abgeleitet. Die prozessorientierte Trainingsforschung hat durch jahrzehntelange Forschung insbesondere durch Untersuchung von Teilzusammenhängen zahlreiche Transferdeterminanten identifiziert, die sich über den Trainings- und Transferprozess hinweg wechselseitig beeinflussen und so den Trainings- und Transfererfolg bedingen können. Anhand von Analysen, die Effekte aller umfassend bestätigten Transferdeterminanten in einem Studiendesign kontrolliert um zentrale konfundierende Einflüsse von Teilnehmenden, Trainings- und Organisationssetting untersuchten, zeigten spezifische Wirkfaktoren zentrale Bedeutung für den Transferprozess. Diese sind nachfolgend mit bewährten Ansätzen für deren Förderung im Sinne eines systematischen Transfermanagements beschrieben und abschließend in einem Leitfaden aufgelistet. Die Maßnahmen sind dabei als Umsetzungsbeispiele aus einem wohl unbegrenzten Möglichkeitsraum der Transferförderung zu verstehen.

Unter Vorbehalt der diskutierten Limitationen können aus dem vorliegenden Forschungsprojekt zentrale Wirkmechanismen aus Forschungsstand und empirischer Prüfung im Kontext von Führungskräftetrainings zur Vermittlung interaktionaler Kompetenzen abgeleitet werden: Die primären Transferdeterminanten Kompetenzentwicklung und Transfermotivation nach dem Training können maßgeblich von ihren Ausgangswerten Kompetenz und Transfermotivation vor dem Training vorhergesagt werden. Zur Förderung motivationaler Wirkmechanismen kann einerseits an den Teilnehmendenmerkmalen Lern- und Trainingsmotivation sowie der Erwartungsklarheit frühzeitig angesetzt werden. Andererseits zeigten auch die Arbeitsumgebungsfaktoren erwartete Vorgesetztenunterstützung, Transferkonsequenzen und -kapazität bereits vor dem Training motivationsförderliche Effekte. Zudem wirken diese Arbeitsumgebungsfaktoren auch zusammen mit zentralen Wirkfaktoren einer transferförderlichen Trainingsgestaltung durch Transferdesign und Inhaltsrelevanz auf die Anwendungsmöglichkeitserwartung. Diese kann zusammen mit erwarteter Mitarbeiter- bzw. Kollegenunterstützung die situative primäre Transferdeterminante realisierte Transfermöglichkeit in der Transferzeit nach Trainings erklären. Diese Wirkmechanismen sollten gezielt durch Transfermanagementmaßnahmen vor, während und nach Trainings gefördert werden. 
Transfermanagementmaßnahmen vor Trainings sollten sich auf eine Bedarfsanalyse zur Spezifizierung der Kompetenz- und Arbeitsanforderungen unter Berücksichtigung der mittelfristigen Unternehmensstrategie durch Organisations- und Aufgabenanalysen konzentrieren. Zur Erfassung eignen sich Interviews mit der Maßnahmenzielgruppe sowie mit deren wichtigen Interaktionspartnern. Zudem kann Auf dieser Basis kann die Trainingskonzeption auf eine transferförderliche Trainingsgestaltung und dadurch die Förderung der Anwendungsmöglichkeitserwartung effizient ausgerichtet werden. Zudem sollten die oben zusammengefassten Teilnehmendenmerkmale und Arbeitsumgebungsfaktoren und insbesondere die Ausgangswerte der primären Transferdeterminanten durch Pre-Traininginterventionen gefördert werden. Neben Vorinformation der Teilnehmenden bezüglich Relevanz, Inhalt und Ablauf, was zusätzlich durch Promotoren des Unternehmens beworben werden sollte, bewährten sich hierfür insbesondere Vorbefragungen bezüglich des Kompetenzniveaus und relevanter Transferdeterminanten im Rahmen der prozessbegleitenden Evaluation sowie eine Einführungsveranstaltung einige Wochen vor dem eigentlichen Training. Ergänzend können weitere Maßnahmen zur Vorbereitung bzw. Einbezug der Vorgesetzten zur Förderung der Arbeitsumgebungsfaktoren eingesetzt werden (siehe Kauffeld et al., 2009; Kauffeld et al., 2012).

Transfermanagementmaßnahmen während Trainings sollten eine auf die Trainingsziele abgestimmte effektive und fundierte Trainingsstrategie fokussieren. Für Trainings zur Vermittlung interaktionaler Kompetenzen bewährte sich der methodische Schwerpunkt von Behavior Modeling Sequenzen (mit positiven und negativen Beispielen und Videofeedback). Dadurch können nicht nur transferrelevante Lerntheorien und Ansätze praxistauglich integriert und umgesetzt werden, sondern auch Lernorientierung und Selbstwirksamkeit sowie weitere spezifische Aspekte der Checkliste für Maßnahmen während Trainings von Salas et al. (2012) effektiv gefördert werden.

Transfermanagementmaßnahmen nach Trainings sollten Post-Traininginterventionen zur Reflexion von Anwendungsmöglichkeiten und Beseitigung potenzieller Transferhindernisse einsetzten. Zudem bewährten sich als zentrale Instrumente, Hilfsmittel und Handlungsempfehlungen autodidaktisch gestaltete Trainingsunterlagen zur weiteren Kompetenzentwicklung und Nachverfolgung nach dem Training. Für Anregung zu Reflexion und Erfahrungsaustausch bezüglich Umsetzung von Trainingsinhalten im Arbeitsalltag scheint es empfehlenswert Maßnahmen vor allem im angeleiteten Format umzusetzen. Eigenverantwortlicher informeller Austausch zu Entwicklungszielen und Transfererfahrungen scheint im herausfordernden Arbeitsalltag eher unterzugehen. Insgesamt zeigte sich auch die prozessbegleitende Evaluation nicht nur vor, sondern auch unmittelbar nach den Trainings sowie nach einer längeren Transferzeit als entscheiden- 
des Transfermanagementinstrument. Einerseits weil es durch Reflexion von Kompetenzentwicklung, Transfermotivation und -möglichkeit förderlich wirkte. Andererseits, weil darauf basierende Datenanalysen ${ }^{47}$ eine optimierte Ausrichtung und Anpassung des Trainings- und Transfermanagementprozesses ermöglichen. Jedoch muss hier auf Einsatz möglichst kurzer und einfacher Fragebögen geachtet werden.

Die hier abgeleiteten Handlungsimplikationen sind nachfolgend in einem Leitfaden für systematisches Transfermanagement zusammengefasst.

${ }^{47}$ Hierbei muss auf Wahrung der Anonymität geachtet werden, weshalb Analysen ausschließlich in zusammengefasster Form ohne Rückführung auf Einzelpersonen durchgeführt werden sollten. 


\section{Zentrale Transferdeterminanten und Maßnahmen, die bereits vor Trainings wirken}

\section{Teilnehmendenmerkmale}

- Lern- und Trainingsmotivation

- Erwartungsklarheit bezüglich Trainingsnutzen

- Ausgangswerte von Kompetenz und Transfermotivation

\section{Arbeitsumgebung}

- Transferunterstützung von Vorgesetzten und Kollegen

- Erwartete Transferkonsequenzen

- Transferkapazität bzw. Zeit zur Vor- und Nachbereitung des Trainings

\section{Maßnahmen systematischen Transfermanagements}

$\rightarrow$ Interview- und fragebogenbasierte Bedarfsanalyse für zielgerichtete lern- und transferförderliche Trainingskonzeption (fundiertes Design und Inhaltsrelevanz) sowie Förderung der Trainingsakzeptanz

$\rightarrow$ Pre-Traininginterventionen zur Förderung der zentralen Teilnehmendeneigenschaften: 1) Vorinformation bezüglich Relevanz, Inhalt und Ablauf, 2) Vorbefragungen bezüglich des Kompetenzniveaus und relevanter Transferdeterminanten durch prozessbegleitende Evaluation sowie 3) Einführungsveranstaltung zur Wissensvermittlung und Motivationsförderung vor dem eigentlichen Training

$\rightarrow$ Gegebenenfalls weitere Maßnahmen zur Vorbereitung bzw. Einbezug der Vorgesetzten der Teilnehmenden zur Förderung der Arbeitsumgebungsfaktoren (z. B. Beteiligung an der Bedarfsanalyse, Aufforderung und Anleitung zur Durchführung von Entwicklungsgesprächen, Aufzeigen der Trainingsrelevanz oder Optimierung von Transferfaktoren der Arbeitsumgebung) ${ }^{48}$

48 Diese Handlungsempfehlung konnte im vorliegenden Forschungsprojekt nur indirekt überprüft werden, da alle Führungskräfte auch Teilnehmende waren. 


\section{Zentrale Transferdeterminanten und Maßnahmen, die während Trainings wirken}

Trainingsgestaltung

- Inhaltsrelevanz

- Transferdesign

\section{Teilnehmendenmerkmale}

- Entwicklung von Kompetenz und Transfermotivation

\section{Arbeitsumgebung}

- Erwartete Anwendungsmöglichkeit

\section{Maßnahmen systematischen Transfermanagements}

$\rightarrow$ Hohe Anwendungs- und Transferorientierung (z. B. durch spezifische Praxisbeispiele in Präsentationen, Diskussionen und Rollenspielen, Reflexion von Anwendungsmöglichkeiten und Beseitigung potenzieller Transferhindernisse zur umfassenden Förderung erwarteter Anwendungsmöglichkeit

$\rightarrow$ Effektive und fundierte Trainingsstrategie, die im Sinne situierten Lernens offene Lernaktivitäten, Exploration und Austausch in konkreten Problemsituationen in einer kontextbezogenen sozialen Lernumgebung Kompetenzentwicklung gezielt fördert, wobei für Trainings zur Vermittlung interaktionaler Kompetenzen insbesondere Behavior Modeling Sequenzen (mit positiven und negativen Beispielen und Videofeedback) empfohlen werden

$\rightarrow$ Prozessbegleitende Evaluation zur Reflexion von Kompetenzentwicklung und zentralen Transferdeterminanten 


\section{Zentrale Transferdeterminanten und Maßnahmen, die nach Trainings wirken}

\section{Teilnehmendenmerkmale}

- Entwicklung von Kompetenz und Transfermotivation

\section{Arbeitsumgebung}

- Realisierte Transfermöglichkeit

\section{Maßnahmen systematischen Transfermanagements}

$\rightarrow$ Post-Traininginterventionen zur Förderung von Selbstmanagementkompetenzen bezüglich Transfer gemäß Zielsetzungstheorie (Locke \& Latham, 1990), der Theorie intentionaler Handlungssteuerung (Gollwitzer, 1993, 1999) bzw. Ansätzen zur Rückfallprävention zur Überwindung gewohnter Verhaltensweisen

$\rightarrow$ Autodidaktisch gestaltete Trainingsunterlagen zur weiteren selbstgesteuerten Kompetenzentwicklung und Entwicklungsüberprüfung nach dem Training.

$\rightarrow$ Auffrischungssitzungen mit Anregung zur Reflexion und Erfahrungsaustausch bezüglich Umsetzung von Trainingsinhalten im Arbeitsalltag im angeleiteten Format

$\rightarrow$ Prozessbegleitende Evaluation zur Reflexion von Entwicklungserfolg und Transferdeterminanten auch noch nach einer angemessenen Transferzeit (bei interaktionalen Kompetenzen mehr als 6 Monate) 


\section{$8 \quad$ Fazit und Ausblick}

Seit Jahrzehnten wird das Transferproblem in Wissenschaft und Praxis beklagt und als zentrale Herausforderung der Personalentwicklung gesehen. Die ergebnisorientierte Trainingsforschung weist darauf hin, dass entgegen anhaltender Zweifel aus Wissenschaft und Praxis fundierte Trainings wirksam sein können. Hinweise auf erfolgsrelevante Transferdeterminanten und mögliche Wirkmechanismen liefert die prozessorientierte Trainingsforschung. Allerdings werden die Forschungserkenntnisse zur Lösung des Transferproblems in der Praxis bislang kaum angewendet (Ford et al., 2018; H. M. Hutchins et al., 2010; Weinbauer-Heidel, 2016). Die vorliegende Arbeit zeigte, dass Führungskräftetrainings mit fundierten Transfermanagementmaßnahmen in kurz- und langfristigem Trainings- und Transfererfolg resultieren, welche Wirkmechanismen dahinter liegen und wie diese gezielt gefördert werden können. Dabei wurden Lücken bisheriger Forschung durch ganzheitliche Erfassung von Transferdeterminanten und Erfolgskriterien sowie methodische Vorgehensweise adressiert. Durch Priorisierung bzw. Differenzierung primärer und sekundärer Transferdeterminanten basierend auf Theorien individueller Leistung sowie durch Konkretisierung von Wirkzusammenhängen konnte zusätzlicher Erkenntnisgewinn zur Erklärung, Überprüfung und Förderung von Transfer generiert werden. Die dadurch realisierte Komplexitätsreduktion bisheriger integrativer Transfermodelle kann sowohl ganzheitliche Trainingsforschung als auch fundierte Transfermanagementaktivitäten in der Trainingspraxis erleichtern.

Aus den umfassenden Datenanalysen lässt sich ableiten, dass sich die Befunde und Empfehlungen für transferförderliche Trainingsgestaltung in Führungskräftetrainings wirksam und praktikabel umsetzten lassen. Als konkrete bestätigte Gestaltungsmaßnahmen, die auch von den Teilnehmenden als positiv erlebt wurden, kann insbesondere der Einsatz von Bedarfsanalyse, Behavior Modeling mit Videofeedback, zeitlich verteilten Trainings mit Pre- und Post-Traininginterventionen sowie Reflexionsunterstützung durch eine nutzerfreundliche prozess- und ergebnisorientierte Evaluation gelten. Durch ein systematisches Transfermanagement vor, während und nach Trainings kann mit verhältnismäßig geringem Mehraufwand der Trainingsnutzen nachweislich gesteigert werden. Dabei sollten neben Kompetenzentwicklung, die sich als bedeutendster Prädiktor bestätigte, auch die Transfermotivation ${ }^{49}$ und -möglichkeit in der Transferzeit gezielt gefördert

49 Allerdings waren die Transfereffekte der Transfermotivation hier nicht so stark und robust wie die der anderen beiden primären Transferdeterminanten. Da Transfereffekte der Transfermotivation jedoch durch die Trainingsforschung als sehr gut belegt gilt, wird der hier nicht klar bestätigte Effekt eher auf den Messzeitpunkt (unmittelbar nach dem Training statt während der Transferzeit) und weitere methodische Aspekte zurückgeführt. 
werden. Explorative Ergebnisse weisen darauf hin, dass dafür transferförderliche Teilnehmendenmerkmale, Arbeitsumgebung und insbesondere Trainingsgestaltung wichtig sind, die die Transfermotivation bereits vor dem Training sowie die erwartete Anwendungsmöglichkeit während des Trainings unterstützten. Für nachhaltig wirksame Führungskräftetrainings ist es somit entscheidend, dass Transferförderung als kollektive Aufgabe verstanden wird. Nur durch gemeinsam getragene Verantwortung für nachhaltige Trainingsgestaltung und -nutzung durch Weiterbildungsanbietende bzw. Trainierende und alle Organisationsmitglieder, von der Unternehmensleitung über Führungskräfte bis hin zu Mitarbeitenden, kann der bestmögliche Trainingseffekt erzielt werden. Aufgrund der Limitationen der Ergebnisse, insbesondere bezüglich ihrer internen und externen Validität, sollten die identifizierten Wirkzusammenhänge an Stichproben in weiteren kontrollierten Trainings- und Organisationssettings überprüft werden. Dabei wären Stichprobengrößen hilfreich, die eine ganzheitliche Modellprüfung zulassen. Wobei der Einsatz von Fragebögen bezüglich des Umfangs und vereinfachter Formulierung optimiert werden sollte. Zudem erscheint eine Erfassung der Transfermotivation über die Transferzeit hinweg wichtig. 


\section{Literatur}

Ajzen, I. (1985). From intentions to actions: A theory of planned behavior. In J. Kuhl \& J. Beckmann (Hrsg.), Action-Control: From cognition to behavior (S. 11-39). Heidelberg: Springer.

Ajzen, I. (1991). The theory of planned behavior. Organizational Behavior and Human Decision Processes, 50(2), 179-211. doi:10.1016/0749-5978(91)90020-T

Al-Swidi, A., \& Al Yahya, M. (2017). Training transfer intention and training effectiveness: Assessing the gender differences using multi-group structural equation modelling approach. International Journal of Organizational Analysis, 25(5), 839-860. doi:10.1108/IJOA-07-2016-1043

Alexander, B. K. (2014). A case for change: Assessment of an evidencedbased leadership development program. Dissertation Abstracts International Section A, 74.

Alkin, M. C. (2004). Evaluation roots: Tracins theorists' views and influences. Thousand Oaks, CA: Sage.

Alliger, G. M., Tannebaum, S. I., Bennett, W. J., \& Traver, H. (1997). A meta-analysis of the relations among training criteria. Personnel Psychology, 50(2), 341-358. doi:10.1111/j.1744-6570.1997.tb00911.x.

Alvelos, R., Ferreira, A. I., \& Bates, R. (2015). The mediating role of social support in the evaluation of training effectiveness. European Journal of Training and Development, 39(6), 484-503. doi:10.1108/EJTD-12-2014-0081

Aragon, I. B., \& Valle, R. S. (2013). Does training managers pay off? The International Journal of Human Resource Management, 24(8). doi:10.1080/09585192.2012.725064

Arasanmi, C. N., Wang, W. Y. C., \& Singh, H. (2017). Examining the motivators of training transfer in an enterprise systems context. Enterprise Information Systems, 11(8), 11541172. doi:10.1080/17517575.2016.1177206

Arthur, W. J., Bennett, W. J., Edens, P. S., \& Bell, S. T. (2003). Effectiveness of training in organizations: A meta-analysis of design and evaluation features. Journal of Applied Psychology, 88(2), 234-245. doi:10.1037/0021-9010.88.2.234

Avolio, B. J., Reichard, R. J., Hannah, S. T., Walumbwa, F. O., \& Chan, A. (2009). A metaanalytic review of leadership impact research: Experimental and quasi-experimental studies. The Leadership Quarterly, 20(5), 764-784. doi:10.1016/j.leaqua.2009.06.006

Baldwin, T. T., \& Ford, J. K. (1988). Transfer of training: A review and directions for future research. Personnel Psychology, 41(1), 63-105. doi:10.1111/j.17446570.1988.tb00632.x

Baldwin, T. T., Ford, J. K., \& Blume, B. D. (2009). Transfer of training 1988-2008: An updated review and agenda for future research. International Review of Industrial and Organizational Psychology, 24(2), 41-70. doi:10.1002/9780470745267.ch2

Baldwin, T. T., Ford, J. K., \& Blume, B. D. (2017). The state of transfer of training research: Moving toward more consumer-centric inquiry. Human Resource Development Quarterly, 28(1), 17-28. doi:10.1002/hrdq.21278

Bandura, A. (1977). Social learning theory. Englewood Cliffs, NJ: Prentice-Hall.

Bandura, A. (1986). Social foundations of thought and action: A social cognitive view. Englewood Cliffs, NJ: Prentice-Hall.

Bass, B. M., \& Avolio, B. (1995). MLQ Multifactor Leadership Questionnaire: Technical report. Redwood City, CA: Mind Garden.

Bassi, L., \& McMurrer, D. (2007). Maximizing your return on people. Harvard Business Review, 85(3), 115-123.

Bates, R., Holton, E. F., \& Hatala, J. P. (2012). A revised learning transfer system inventory: Factorial replication and validation. Human Resource Development International, 15(5), 549-569. doi:10.1080/13678868.2012.726872 
Bates, R., Kauffeld, S., \& Holton, E. F. (2008). Examining the factor structure and predictive ability of the German-version of the Learning Transfer Systems Inventory. [Die Faktorstruktur und Vorhersagevalidität der deutschen Version des Learning Transfer Systems Inventory]. Journal of European Industrial Training, 31(3), 195-211. doi:10.1108/03090590710739278

Bauer, K. N., Orvis, K. A., Ely, K., \& Surface, E. A. (2016). Re-examination of motivation in learning contexts: Meta-analytically investigating the role type of motivation plays in the prediction of key training outcomes. Journal of Business \& Psychology, 31(1), 33-50. doi:10.1007/s10869-015-9401-1

Becker, M. (2011). Systematische Personalentwicklung: Planung, Steuerung und Kontrolle im Funktionszyklus (2. Aufl.). Stuttgart: Schäffer-Poeschel.

Becker, M. (2013). Personalentwicklung: Bildung, Förderung und Organisationsentwicklung in Theorie und Praxis (6. Aufl.). Stuttgart: Schäffer-Poeschel.

Beer, M., Finnstrom, M., \& Schrader, D. (2016). Why leadership training fails - and what to do about it. Harvard Business Review, 94(10), 50-57.

Berardinelli, P. K., Burrow, J. L., \& Dillon Jones, L. S. (1995). Management Training: An Impact Theory. Human Resource Development Quarterly, 6(1), 79-90. doi:10.1002/hrdq.3920060107

Bhatti, M. A., Ali, S., Isa, M. F. M., \& Battour, M. M. (2014). Training transfer and transfer motivation: The influence of individual, environmental, situational, training design, and affective reaction factors. Performance Improvement Quarterly, 27(1), 51-82. doi:10.1002/piq.21165

Blumberg, M., \& Pringle, C. D. (1982). The missing opportunity in organizational research: Some implications for a theory of work performance. Academy of Management Review, 7(4), 560-569. doi:10.5465/amr.1982.4285240

Blume, B. D., Ford, J. K., Baldwin, T. T., \& Huang, J. L. (2010). Transfer of training: A metaanalytic review. Journal of Management, 36(4), 1065-1105. doi:10.1177/0149206309352880

Blume, B. D., Ford, J. K., Surface, E. A., \& Olenick, J. (2019). A dynamic model of training transfer. Human Resource Management Review, 29(2), 270-283. doi:10.1016/j.hrmr.2017.11.004

Bortz, J., \& Döring, N. (2009). Forschungsmethoden und Evaluation für Human- und Sozialwissenschaftler (4. Aufl.). Heidelberg: Springer.

Brackett, M. A., Rivers, S. E., Shiffman, S., Lerner, N., \& Salovey, P. (2006). Relating emotional abilities to social functioning: A comparison of self-report and performance measures of emotional intelligence. Journal of Personality and Social Psychology, 91(4), 780-795. doi:10.1037/0022-3514.91.4.780

Brahma, S. S., \& Chakraborty, H. (2019). A Moderated Mediation Analysis of Supportive Work Environment, Motivation to Transfer and Training Transfer in the Small and Medium Retail Firms in Saudi Arabia. IUP Journal of Business Strategy, 16(2), 7-27.

Bramley, P. (1999). Evaluating effective management learning. Journal of European Industrial Training, 23(3), 145-153. doi:10.1108/03090599910261826

Brodgen, H. E. (1949). When testing pays off. Personnel Psychology, 2(2), 171-183. doi:10.1111/j.1744-6570.1949.tb01397.x

Brown, T. C., McCracken, M., \& Hillier, T.-L. (2013). Using evidence-based practices to enhance transfer of training: assessing the effectiveness of goal setting and behavioural observation scales. Human Resource Development International, 16(4), 374-389. doi:10.1080/13678868.2013.812291

Brown, T. C., \& Warren, A. M. (2014). Evaluation of transfer of training in a sample of union and management participants: a comparison of two self-management techniques. Human Resource Development International, 17(3), 277-296.

doi:10.1080/13678868.2014.907975 
Brown, T. C., Warren, A. M., \& Khattar, V. (2016). The effects of different behavioral goals on transfer from a management development program. Human Resource Development Quarterly, 27(3), 349-372. doi:10.1002/hrdq.21257

Bühler, W., \& Siegen, T. (1999). Unternehmenssteuerung und Anreizsysteme. Stuttgart: Schäffer-Poeschel.

Bungard, W., Holling, H., \& Schultz-Gambard, J. (1996). Methoden der Arbeits- und Organisationspsychologie (6. Aufl.). Weinheim: Beltz Psychologie Verlags Union.

Buren, M. v., \& Erskine, W. (2002). The 2002 ASTD state of the industry report. Alexandria: American Society for Training \& Development.

Burke, L. A., \& Hutchins, H. M. (2007). Training transfer: An integrative literature review. Human Resource Development Review, 6(3), 263-296. doi:10.1177/1534484307303035

Burke, L. A., \& Hutchins, H. M. (2008). A study of best practices in training transfer and proposed model of transfer. Human Resource Development Quarterly, 19(2), 107-128. doi:10.1002/hrdq.1230

Burke, L. A., Hutchins, H. M., \& Saks, A. M. (2013). Best practices in training transfer. In M. A. Paludi (Hrsg.), Psychology for business success, Vol. 1. Juggling, balancing, and integrating work and family roles and responsibilities, Vol. 2. Institutional equity and compliance, Vol. 3. Managing, leading, and developing employees, Vol. 4. Implementing best practices in human resources (S. 115-132): Praeger/ABC-CLIO.

Burke, M. J., \& Day, R. R. (1986). A cumulative study of the effectiveness of managerial training. Journal of Applied Psychology, 71(2), 232-245. doi:10.1037/00219010.71.2.232

Campbell, J. P. (1990). Modeling the performance prediction problem in industrial and organizational psychology. In M. D. Dunnette \& L. M. Hough (Hrsg.), Handbook of industrial and organizational psychology, Vol. 1 (S. 687-732). Palo Alto CA: Consulting Psychologists Press.

Cannon-Bowers, J. A., Salas, E., Tannenbaum, S. I., \& Mathieu, J. E. (1995). Toward theoretically based principles of training effectiveness: A model and initial empirical investigation. Military Psychology, 7(3), 141-164. doi:10.1207/s15327876mp0703_1

Cappetta, R., \& Paolino, C. (2015). Is it always worth waiting? The effect of autonomysupportive teaching on short-term and long-term learning outcomes. British Journal of Management, 26(1), 93-108. doi:10.1111/1467-8551.12065

Carlson, K. D., \& Schmidt, F. L. (1999). Impact of experimental design on effect size: Findings from the research literature on training. Journal of Applied Psychology, 84(6), 851-862. doi:10.1037/0021-9010.84.6.851

Cascio, W. F., \& Aguinis, H. (2005). Applied psychology in human resource management (6. Aufl.). Upper Saddle River, NJ: Prentice Hall.

Chauhan, R., Ghosh, P., Rai, A., \& Kapoor, S. (2017). Improving transfer of training with transfer design: Does supervisor support moderate the relationship? Journal of Workplace Learning, 29(4), 268-285. doi:10.1108/JWL-08-2016-0079

Chauhan, R., Ghosh, P., Rai, A., \& Shukla, D. (2016). The impact of support at the workplace on transfer of training: A study of an indian manufacturing unit. International Journal of Training and Development, 20(3), 200-213. doi:10.1111/ijtd.12083

Chen, H.-T. (1990). Theory-driven evaluations. Newbury Park, CA: Sage.

Cheng, E. W. L., \& Hampson, I. (2008). Transfer of training: A review and new insights. International Journal of Management Reviews, 10(4), 327-341. doi:10.1111/j.14682370.2007.00230.x.

Cheng, E. W. L., \& Ho, D. C. K. (2001). A review of transfer of training studies in the past decade. Personnel Review, 30(1), 102-118. doi:10.1108/00483480110380163

Cheng, E. W. L., Sanders, K., \& Hampson, I. (2015). An intention-based model of transfer of training. Management Research Review, 38(8), 908-928. doi:10.1108/MRR-05-20140107 
Chiaburu, D. S., Dam, K. v., \& Hutchins, H. M. (2010). Social Support in the Workplace and Training Transfer: A longitudinal analysis. International Journal of Selection \& Assessment, 18(2), 187-200. doi:10.1111/j.1468-2389.2010.00500.x

Clark, L. S. (1982). An analysis of changes in attitudes of supervisors and managers toward supervisory practices, company policies, and supervisor opinions (Doctoral dissertation). ABI/INFORM Complete; ProQuest Dissertations \& Theses A\&I; ProQuest Dissertations \& Theses Global. (8225498).

Cohen, J. (1988). Statistical power analysis for the behavioural sciences (2. Aufl.). New York, NY: Academic Press.

Cohen, J. (1992). A power primer. Psychological Bulletin, 112(1), 155-159.

Collani, G. v., \& Herzberg, P. Y. (2003). Zur internen Struktur des globalen Selbstwertgefühls nach Rosenberg. Zeitschrift für Differentielle und Diagnostische Psychologie, 24(1), 922. doi:10.1024//0170-1789.24.1.9

Collins, D. B., \& Holton, E. F. (2004). The effectiveness of managerial leadership development programs: A meta-analysis of studies from 1982 to 2001 . Human Resource Development Quarterly, 15(2), 217-248. doi:10.1002/hrdq.1099

Colquitt, J. A., LePine, J. A., \& Noe, R. A. (2000). Toward an integrative theory of training motivation: A meta-analytic path analysis of 20 years of research. Journal of Applied Psychology, 85(5), 678-707. doi:10.1037/0021-9010.85.5.678

Conradi, W. (1983). Personalentwicklung. Stuttgart: Enke.

Cortina, J. M. (1993). What is coefficient alpha? An examination of theory and applications. Journal of Applied Psychology, 78(1), 98-104. doi:10.1037/0021-9010.78.1.98

Cortina, K. S., \& Pant, H. A. (2009). Hierarchische Lineare Modelle (HLM). In H. Holling (Hrsg.), Grundlagen und statistische Methoden der Evaluationsforschung (S. 335-362). Göttingen: Hogrefe.

Creon, L. E., \& Schermuly, C. C. (2019). Training group diversity and training transfer: A psychological safety perspective. Human Resource Development Quarterly, 30(4). doi:10.1002/hrdq.21372

Creusen, U. (2012). Zum Glück gibt es Erfolg durch Positive Leadership. HR Performance, 1.

Cromwell, S. E., \& Kolb, J. A. (2004). An examination of work-environment support factors affecting transfer of supervisory skills training to the workplace. Human Resource Development Quarterly, 15(4), 449-471. doi:10.1002/hrdq.1115

Cronbach, L. J. (1951). Coefficient alpha and the internal structure of tests. Psychometrika, 16(3), 297-334. doi:10.1007/BF02310555

Cronbach, L. J. (1982). Designing evaluations of educational and social programs. San Francisco: Jossey-Bass.

Cronbach, L. J., \& Gleser, G. C. (1965). Psychological tests and personnel decisions (2. Aufl.). Urbana: University of Illinois Press.

Culpin, V., Eichenberg, T., Hayward, I., \& Abraham, P. (2014). Learning, intention to transfer and transfer in executive education. International Journal of Training and Development, 18(2), 132-147. doi:10.1111/ijtd.12033

Curado, C., Henriques, P. L., \& Ribeiro, S. (2015). Voluntary or mandatory enrollment in training and the motivation to transfer training. International Journal of Training and Development, 19(2), 98-109. doi:10.1111/ijtd.12050

Dalal, R. S., Bhave, D. P., \& Fiset, J. (2014). Within-person variability in job performance: A theoretical review and research agenda. Journal of Management, 40(5), 1396-1436. doi:10.1177/0149206314532691

Dalecki, M., \& Willits, F. K. (1991). Examining change using regression analysis: Three approaches compared. Socialogical Spectrum, 11(2), 127-145. doi:10.1080/02732173.1991.9981960 
Day, D. V., Zaccaro, S. J., \& Halpin, S. M. (2005). Leader development for transforming organizations: Growing leaders for tomorrow. Mahwah, NJ: Lawrence Erlbaum Associates.

DeGEval. (2016). Standards für Evaluationen. Verfügbar unter http://www.degeval.de/images/stories/Publikationen/DeGEval - Standards.pdf (26.02.2019).

Diamantidis, A. D., \& Chatzoglou, P. D. (2014). Employee post-training behaviour and performance: Evaluating the results of the training process. International Journal of Training and Development, 18(3), 149-170. doi:10.1111/ijtd.12034

Diebig, M., Bormann, K. C., \& Rowold, J. (2016). A double-edged sword: Relationship between full-range leadership behaviors and followers' hair cortisol level. The Leadership Quarterly, 27(4), 684-696. doi:10.1016/j.leaqua.2016.04.001

Diesner, I., \& Seufert, S. (2010). Trendstudie 2010: Herausforderungen für das Bildungsmanagement in Unternehmen. Swiss Competence Centre for Innovations in Learning.

Diesner, I., \& Seufert, S. (2013). Trendstudie 2012: Herausforderungen für das Bildungsmanagement in Unternehmen. Swiss Competence Centre for Innovations in Learning.

Dubs, R. (1990). Lernprozesse in Unternehmungen beschleunigen - Zur Transferproblematik in Unternehmungen. Schweizerische Zeitschrift für betriebswirtschaftliche Forschung und Praxis, 90(2), 154-163.

Dulebohn, J., Bommer, W. H., Liden, R. C., Brouer, R. L., \& Ferris, G. R. (2012). A metaanalysis of antecedents and consequences of Leader-Member Exchange: Integrating the past with an eye toward the future. Journal of Management, 38(6), 1715-1759. doi:10.1177/0149206311415280

Dweck, C. S. (1986). Motivational processes affecting learning. American Psychology, 41(10), 1040-1048. doi:10.1037/0003-066X.41.10.1040

Egan, T. M. (2008). The relevance of organizational subculture for motivation to transfer learning. Human Resource Development Quarterly, 19(4), 299-322. doi:10.1002/hrdq.1243

Eichenberger, P. C. (1990). Millionen für Bildung, Pfennige für Evaluation. Personalwirtschaft, 3 , 35-43.

Enders, C. K. (2010). Applied missing data analysis. New York, NY [u.a.]: Guilford Press.

Enos, M. D., Kehrhahn, M. T., \& Bell, A. (2003). Informal learning and the transfer of learning: How managers develop proficiency. Human Resource Development Quarterly, 14(4), 369-387. doi:10.1002/hrdq.1074

Eraut, M. (2004). Transfer of knowledge between education and workplace settings. In $\mathrm{H}$. Rainbird, A. Fuller, \& A. Munro (Hrsg.), Workplace Learning in Context (S. 201-222). London: Routledge.

Evaluation, J. C. o. S. f. E. (2011). The program evaluation standards (3. Aufl.). Los Angeles, CA: Sage.

Facteau, J. D., Dobbins, G. H., Russell, J. E. A., Ladd, R. T., \& Kudisch, J. D. (1995). The influence of general perceptions of the training environment on pretraining motivation and perceived training transfer. Journal of Management, 21(1), 1-25.

Fandel-Meyer, T., Schneider, C., Seufert, S., Meier, C., \& Schuchmann, D. (2015). Trendstudie 2015/2016: Trends im Corporate Learning. Swiss Competence Centre for Innovations in Learning.

Felfe, J. (2006). Validierung einer deutschen Version des „Multifactor Leadership Questionnaire” (MLQ 5 X Short) von Bass und Avolio (1995). Zeitschrift für Arbeits- und Organisationspsychologie, 50(2), 61-78. doi:10.1026/0932-4089.50.2.61

Felfe, J. (2016). Handbuch Mitarbeiterführung: Wirtschaftspsychologisches Praxiswissen für Fach- und Führungskräfte. Berlin: Springer. 
Felfe, J., \& Franke, F. (2014). Führungskräftetrainings. Göttingen [u.a.]: Hogrefe.

Felfe, J., \& Goihl, K. (2002). Deutsche überarbeitete und ergänzte Version des „Multifactor Leadership Questionnaire" (MLQ). In A. Glöckner-Rist (Hrsg.), ZUMAInformationssystem. Elektronisches Handbuch sozialwissenschaftlicher Erhebungsinstrumente. Version 6.00. Mannheim: ZUMA.

Field, A. (2018). Discovering statistics using IBM SPSS statistics (5. Aufl.). Los Angeles [u. a.]: Sage.

Fisher, G. G., Matthews, R. A., \& Gibbons, A. M. (2016). Developing and investigating the use of single-item measures in organizational research. Journal of Occupational Health Psychology, 21(1), 3-23. doi:10.1037/a0039139

Fleishman, E. A. (1953). Leader climate, human relations training and supervisory behavior. Personnel Psychology, 6(2), 205-222. doi:10.1111/j.1744-6570.1953.tb01040.x

Ford, J. K., Baldwin, T. T., \& Prasad, J. (2018). Transfer of training: The known and the unknown. Annual Review of Organizational Psychology and Organizational Behavior, 5, 201-225. doi:10.1146/annurev-orgpsych-032117-104443

Freitas, A. C., Silva, S. A., \& Santos, C. M. (2019). Safety training transfer: The roles of coworkers, supervisors, safety professionals, and felt responsibility. Journal of Occupational Health Psychology, 24(1), 92-107. doi:10.1037/ocp0000125

Frese, M., Beimel, S., \& Schoenborn, S. (2003). Action training for charismatic leadership: Two evaluations of studies of a commercial training module on inspirational communication of a vision. Personnel Psychology, 56(3), 671-697. doi:10.1111/j.17446570.2003.tb00754.x

Gagne, R. M. (1965). The conditions of learning. New York, NY: Holt, Rinehart, and Winston.

Gegenfurtner, A. (2011). Motivation and transfer in professional training: A meta-analysis of the moderating effects of knowledge type, instruction, and assessment conditions. Educational Research Review, 6(3), 153-168. doi:10.1016/j.edurev.2011.04.001

Gegenfurtner, A., Könings, K. D., Kosmajac, N., \& Gebhardt, M. (2016). Voluntary or mandatory training participation as a moderator in the relationship between goal orientations and transfer of training. International Journal of Training and Development, 20(4), 290-301.

Gegenfurtner, A., Veermans, K., Festner, D., \& Gruber, H. (2009). Motivation to transfer training: An integrative literature review. Human Resource Development Review, 8(3), 403-423. doi:10.1177/1534484309335970

Gegenfurtner, A., Veermans, K., \& Vauras, M. (2013). Effects of computer support, collaboration, and time Lag on performance self-efficacy and transfer of training: A longitudinal meta-analysis. Educational Research Review, 8, 75-89. doi:10.1016/j.edurev.2012.04.001

Gessler, M. (2012). Lerntransfer in der beruflichen Weiterbildung - empirische Prüfung eines integrierten Rahmenmodells mittels Strukturgleichungsmodellierung. Zeitschrift für Berufs- und Wirtschaftspädagogik, 108(3), 362-393.

Gick, M. L., \& Holyoak, K. J. (1987). The cognitive basis of knowledge transfer. In S. M. Cormier \& J. D. Hagman (Hrsg.), Transfer of learning. New York, NY: Academic Press.

Goldstein, I. L., \& Ford, J. K. (2002). Training in organizations needs assessment, development, and evaluation (4. Aufl.). Belmont, CA u.a.: Wadsworth.

Gollwitzer, P. M. (1993). Goal achievement: The role of intentions. European Review of Social Psychology, 4, 141-185.

Gollwitzer, P. M. (1999). Implementation intentions: Strong effects of simple plans. American Psychologist, 54, 493-503.

Govaerts, N., \& Dochy, F. (2014). Disentangling the role of the supervisor in transfer of training. Educational Research Review, 12, 77-93. doi:10.1016/j.edurev.2014.05.002

Govaerts, N., Kyndt, E., \& Dochy, F. (2018). The influence of specific supervisor support types on transfer of training: Examining the mediating effect of training retention. Vocations and Learning, 11(2), 265-288. doi:10.1007/s12186-017-9190-y 
Govaerts, N., Kyndt, E., Vreye, S., \& Dochy, F. (2017). A supervisors' perspective on their role in transfer of training. Human Resource Development Quarterly, 28(4), 515-552. doi:10.1002/hrdq.21286

Graen, G. B., \& Uhl-Bien, M. (1995). Relationship-based approach to leadership: Development of leader-member exchange (LMX) theory of leadership over 25 years: Applying a multilevel multi-domain perspective. The Leadership Quarterly, 6(2), 219-247. doi:10.1016/1048-9843(95)90036-5

Grohmann, A., Beller, J., \& Kauffeld, S. (2014). Exploring the critical role of motivation to transfer in the training transfer process. International Journal of Training and Development, 18(2), 84-103. doi:10.1111/ijtd.12030

Grohmann, A., \& Kauffeld, S. (2013). Evaluating training programs: Development and correlates of the Questionnaire for Professional Training Evaluation. International Journal of Training and Development, 17(2), 135-155. doi:10.1111/ijtd.12005

Grossman, R., \& Burke-Smalley, L. A. (2018). Context-dependent accountability strategies to improve the transfer of training: A proposed theoretical model and research propositions. Human Resource Management Review, 28(2), 234-247. doi:10.1016/j.hrmr.2017.08.001

Groves, R. M., Dillman, D. A., Eltinge, J. L., \& Little, R. A. J. (2002). Survey nonresponse. Hoboken: Wiley.

Groves, R. M., Fowler Jr., F. J., Couper, M. P., \& Lepkowski, J. M. (2009). Survey methodology. Hoboken: Wiley.

Hair, J. F. (2014). Multivariate data analysis (7. Aufl.). Harlow: Pearson.

Hammer, L. B., Truxillo, D. M., Bodner, T., Pytlovany, A. C., \& Richman, A. (2019). Exploration of the impact of organisational context on a workplace safety and health intervention. Work \& Stress, 33(2), 192-210. doi:10.1080/02678373.2018.1496159

Hart, S. L., Steinheider, B., \& Hoffmeister, V. E. (2019). Team-Based Learning and training transfer: A case study of training for the implementation of Enterprise Resources Planning Software. International Journal of Training and Development, 23(2), 135-152. doi:10.1111/ijtd.12150

Hasselhorn, M., \& Mähler, C. (2000). Transfer: Theorien, Technologien und empirische Erfassung. In W. Hager, J.-L. Patry, \& H. Brezing (Hrsg.), Evaluation psychologischer Interventionsmaßnahmen. Standards und Kriterien: Ein Handbuch (S. 86-101 ). Bern: Huber.

Hinrichs, A.-C. (2014). Predictors of collateral learning transfer in continuing vocational training. International Journal for Research in Vocational Education and Training, 1(1), 35-56. doi:10.13152/IJRVET.1.1.3

Hinrichs, A.-C. (2016). Erfolgsfaktoren beruflicher Weiterbildung. Eine längsschnittstudie zum Lerntransfer. Wiesbaden: Springer.

Ho, M. (2016). Investment in learning increases for fourth straight year. Verfügbar unter https://www.td.org/magazines/td-magazine/investment-in-learning-increases-for-fourthstraight-year (26.09.2019).

Hochholdinger, S., Rowold, J., \& Schaper, N. (2008). Ansätze zur Trainings- und Transferevaluation. In J. Rowold, S. Hochholdinger, \& N. Schaper (Hrsg.), Trainingsevaluation und Transfersicherung: Modelle, Methoden, Befunde (S. 30-52). Göttingen: Hogrefe.

Holdnak, B. J., Clemons, T. C., \& Bushardt, S. C. (1990). Evaluation of organisation training by the Solomon Four Group design: A field study in self-esteem training. Journal of Managerial Psychology, 5(5), 25-31. doi:10.1108/02683949010136251

Holton, E. F. (1996). The flawed four-level evaluation model. Human Resource Development Quarterly, 7(1), 5-21. doi:10.1002/hrdq.3920070103

Holton, E. F. (2005). Holton's evaluation model: New evidence and construct elaborations. Advances in Developing Human Resources, 7(1), 37-54. doi:10.1177/1523422304272080 
Holton, E. F., \& Bates, R. A. (1997). Toward construct validation of a transfer climate instrument. Human Resource Development Quarterly, 8(2), 95-113. doi:10.1002/hrdq.3920080203

Holton, E. F., Bates, R. A., \& Ruona, W. E. A. (2000). Development of a generalized learning transfer system inventory. Human Resource Development Quarterly, 11(4), 333-360. doi:10.1002/1532-1096(200024)11:4<333::AID-HRDQ2>3.0.CO;2-P

Holton, E. F., Hsin-Chih, C., \& Naquin, S. S. (2003). An examination of learning transfer system characteristics across organizational settings. Human Resource Development Quarterly, 14(4), 459-482. doi:10.1002/hrdq.1079

Homklin, T., Takahashi, Y., \& Techakanont, K. (2014). The influence of social and organizational support on transfer of training: Evidence from Thailand. International Journal of Training and Development, 18(2), 116-131. doi:10.1111/ijtd.12031

Huang, J. L., Blume, B. D., Ford, J. K., \& Baldwin, T. T. (2015). A tale of two transfers: Disentangling maximum and typical transfer and their respective predictors. Journal of Business \& Psychology, 30(4), 709-732. doi:10.1007/s10869-014-9394-1

Hughes, A. M., Gregory, M. E., Joseph, D. L., Sonesh, S. C., Marlow, S. L., Lacerenza, C. N., . . . Salas, E. (2016). Saving lives: A meta-analysis of team training in healthcare. Journal of Applied Psychology, 101(9), 1266-1304. doi:10.1037/apl0000120

Hughes, A. M., Zajac, S., Woods, A. L., \& Salas, E. (2019). The role of work environment in training sustainment: A meta-analysis. Human Factors, 62(1), 166-183. doi:10.1177/0018720819845988

Huint, P., \& Saks, A. M. (2003). Translating training science into practice: A study of managers' reactions to posttraining transfer interventions. Human Resource Development Quarterly, 14(2), 181-198. doi:10.1002/hrdq.1059

Hülshoff, T., Negri, C., Hüther, G., Dohne, K.-D., Hoffmann, C., \& Kalt, M. (2010). Lernpsychologie. In C. Negri (Hrsg.), Angewandte Psychologie für die Personalentwicklung. Konzepte und Methoden für Bildungsmanagement, betriebliche Aus- und Weiterbildung (S. 69-113). Berlin: Springer.

Hunter, J. E., \& Schmidt, F. L. (1990). Methods of meta-analysis. Thousand Oaks, CA: Sage.

Hunter, J. E., \& Schmidt, F. L. (2004). Methods of meta-analysis: correcting error and bias in research findings (2. Aufl.). Thousand Oaks, CA: Sage.

Hunter, J. E., Schmidt, F. L., \& Jackson, G. B. (1982). Meta-analysis. Beverly Hills, CA: Sage.

Hutchins, H. M., Burke, L. A., \& Berthelsen, A. M. (2010). A missing link in the transfer problem? Examining how trainers learn about training transfer. Human Resource Management, 49(4), 599-618. doi:10.1002/hrm.20371

Hutchins, H. M., Nimon, K., Bates, R. A., \& Holton, E. F. (2013). Can the LTSI predict transfer performance? Testing intent to transfer as a proximal transfer of training outcome. International Journal of Selection \& Assessment, 21(3), 251-263. doi:10.1111/ijsa.12035

Hutchins, S. D., Wickens, C. D., Carolan, T. F., \& Cumming, J. M. (2013). The influence of cognitive load on transfer with error prevention training methods: A meta-analysis. Human Factors, 55(4), 854-874. doi:10.1177/0018720812469985

Iddekinge, C. H. v., Aguinis, H., Mackey, J. D., \& DeOrtentiis, P. S. (2018). A meta-analysis of the interactive, additive, and relative effects of cognitive ability and motivation on performance. Journal of Management, 44(1), 249-279. doi:10.1177/0149206317702220

Iqbal, K., \& Dastgeer, G. (2017). Impact of self-efficacy and retention on transfer of training: The mediating role of motivation to transfer. Journal of Management Development, 36(10), 1270-1282. doi:10.1108/JMD-06-2015-0087

Islam, T., \& Ahmed, I. (2018). Mechanism between perceived organizational support and transfer of training. Management Research Review, 41(3), 296-313. doi:10.1108/MRR02-2017-0052 
Jeung, C.-W., Yoon, H. J., Park, S., \& Jo, S. J. (2011). The contributions of human resource development research across disciplines: A citation and content analysis. Human Resource Development Quarterly, 22(1), 87-109. doi:10.1002/hrdq.20062

Johnson, S. J., Blackman, D. A., \& Buick, F. (2018). The 70:20:10 framework and the transfer of learning. Human Resource Development Quarterly, 29(4), 383-402. doi:10.1002/hrdq. 21330

Judd, C. (1908). The relation of special training and general intelligence. Educational Review, $36,42-48$.

Kaiser, R. B., \& Curphy, G. (2013). Leadership development: The failure of an industry and an opportunity for consulting psychologists. Consulting Psychology Journal: Practice and Research, 65(4), 294-302. doi:10.1037/a0035460

Kanning, U. P. (2014). Prozess und Methoden der Personalentwicklung. In H. Schuler \& U. P. Kanning (Hrsg.), Lehrbuch der Personalpsychologie (3. Aufl., S. 501-562). Göttingen: Hogrefe.

Kanning, U. P., \& Thielsch, M. T. (2015). Wie bilden Personalpraktiker/innen sich weiter? Zeitschrift für Arbeits- und Organisationspsychologie, 59(4), 206-214. doi:10.1026/09324089/a000195

Kauffeld, S. (2006). Kompetenzen messen, bewerten, entwickeln - Ein prozessanalytischer Ansatz für Gruppen. Stuttgart: Schäffer-Poeschel.

Kauffeld, S. (2016). Nachhaltige Personalentwicklung und Weiterbildung: Betriebliche Seminare und Trainings entwickeln, Erfolge messen, Transfer sichern (2. Aufl.). Berlin: Springer.

Kauffeld, S., Bates, R., Holton, E. F., \& Müller, A. C. (2008). Das deutsche Lerntransfer-SystemInventar (GLTSI): Psychometrische Überprüfung der deutschsprachigen Version. Zeitschrift für Personalpsychologie, 7(2), 50-69. doi:10.1026/1617-6391.7.2.50

Kauffeld, S., Grote, S., \& Frieling, E. (2009). Kompetenzentwicklung jenseits der Schulbank aber wie? In S. Kauffeld, S. Grote, \& E. Frieling (Hrsg.), Handbuch Kompetenzentwicklung (S. 1-10): Schäffer-Poeschel Verlag für Wirtschaft Steuern Recht $\mathrm{GmbH}$.

Kauffeld, S., Lorenzo, G., \& Weisweiler, S. (2012). Wann wird Weiterbildung nachhaltig? Erfolg und Erfolgsfaktoren beim Lerntransfer. Personal Quarterly, 64(2), 10-15.

Kehr, H. M., Bles, P., \& Rosenstiel, L. v. (1999). Zur Motivation von Führungstkräften: Zielbindung und Flusserleben als transferfördernde Faktoren bei Führungskräftetrainings. Zeitschrift für Arbeits- und Organisationspsychologie, 42, 8394.

Keith, N., \& Frese, M. (2008). Effectiveness of Error Management Training: A meta-analysis. Journal of Applied Psychology, 93(1), 59-69. doi:10.1037/0021-9010.93.1.59

Kellner, H. J. (2006). Value of investment: Neue Evaluierungsmethoden für Personalentwicklung und Bildungscontrolling. Offenbach: GABAL.

Kim, S., Park, S., Lavelle, J., Kim, M., \& Chaudhuri, S. (2019). Revisiting trainee reactions: A multilevel analysis of the nomological network. Human Resource Development Quarterly. doi:10.1002/hrdq.21383

Kim, Y. H., Ong, M., \& Chung, J. H. (2014). Enhancing training motivation in the public sector in South Korea: the value of a contingency approach. Asia Pacific Journal of Public Administration, 36(4), 301-312. doi:10.1080/23276665.2014.999398

Kirkpatrick, D. L. (1967). Evaluation of training. In R. L. Craig (Hrsg.), Training and development handbook: A guide to human resources development (S. 87-112). New York, NY: McGraw-Hill.

Kirkpatrick, D. L. (1994). Evaluating training programs. San Francisco: Berrett-Koehler Publishers.

Kirkpatrick, D. L. (1998). Evaluating training programs (2. Aufl.). San Francisco: Berrett-Koehler.

Kline, P. (1999). The handbook of psychological testing (2. Aufl.). London: Routledge. 
Kodwani, A. D. (2017). Decoding training effectiveness: The role of organisational factors. Journal of Workplace Learning, 29(3), 200-216. doi:10.1108/JWL-05-2016-0038

Kodwani, A. D., \& Prashar, S. (2019a). Assessing the influencers of sales training effectiveness before and after training: Mediating role of motivation to learn and moderating role of choice. Benchmarking: An International Journal, 26(4), 1233-1254. doi:10.1108/BIJ-052018-0126

Kodwani, A. D., \& Prashar, S. (2019b). Exploring the influence of pre-training factors on training effectiveness-moderating role of trainees' reaction: a study in the public sector in India. Human Resource Development International, 22(3), 283-304. doi:10.1080/13678868.2019.1596012

Kolb, M. (2010). Führen mit Zielen - ein wiederentdecktes und bewährtes Haus- bzw. (All)Heilmittel?! In M. Schwaab, G. Bergmann, F. Gairing, \& M. Kolb (Hrsg.), Führen mit Zielen (S. 3-19). Wiesbaden: Gabler Verlag.

Kozlowski, S. W. J., Watola, D. J., Jensen, J. M., Kim, B. H., \& Botero, I. C. (2009). Developing adaptive teams: A theory of dynamic team leadership. In E. Salas, G. F. Goodwin, \& C. S. Burke (Hrsg.), Team effectiveness in complex organizations: Cross-disciplinary perspectives and approaches (S. 113-155). New York, NY: Routledge Academic.

Kraiger, K. (2002). Decision-based evaluation. In K. Kraiger (Hrsg.), Creating, implementing and managing effective training and development state-of-the-art lessons for practice (S. 331-375). San Francisco, CA: Jossey-Bass.

Krampen, G., \& Hank, P. (2008). Prozessdiagnostik und kontrollierte Praxis. In B. Röhrle, F. Caspar, \& P. F. Schlottke (Hrsg.), Lehrbuch der klinisch-psychologischen Diagnostik (S. 300-329). Stuttgart: Kohlhammer.

Lacerenza, C. N., Reyes, D. L., \& Marlow, S. L. (2017). Leadership training design, delivery, and implementation: A meta-analysis. Journal of Applied Psychology, 102(12), 16861718. doi:10.1037/apl0000241

Lai, L. C. (1996). A meta-analysis of research on the effectiveness of leadership training programs. Unveröffentlichte Dissertation. University of Wisconsin-Madison.

Laker, D. R. (1990). Dual dimensionality of training transfer. Human Resource Development Quarterly, 1(3), 209-223. doi:10.1002/hrdq.3920010303

Laker, D. R., \& Powell, J. L. (2011). The differences between hard and soft skills and their relative impact on training transfer. Human Resource Development Quarterly, 22(1), 111-122. doi:10.1002/hrdq.20063

Lauzier, M., \& Mercier, G. (2018). The effect of error orientation, motivation to learn, and social support on training transfer intentions: A moderated mediation model. Canadian Journal of Administrative Sciences, 35(3), 419-428. doi:10.1002/cjas.1429

Lave, J., \& Wenger, E. (1991). Situated learning: Legitimate peripheral participation. New York: Cambridge University Press.

Lee, C., Jeon, D., Kim, W., \& Lee, J. (2017). Evaluating training for new government officials: A case study using the success case method. Public Personnel Management, 46(4), 419444. doi:10.1177/0091026017730382

Lee, C., Lee, H., Lee, J., \& Park, J. (2014). A multiple group analysis of the training transfer model: exploring the differences between high and low performers in a Korean insurance company. International Journal of Human Resource Management, 25(20), 2837-2857. doi:10.1080/09585192.2014.934887

Lemke, S. G. (1995). Transfermanagement. Göttingen: Verlag für Angewandte Psychologie.

Li, Y., \& Wiriyakitjar, R. (2018). Dominant factors influencing training effectiveness (learning and transfer performance) of SMEs in Beijing, China: A study on the hotel sector. UTCC International Journal of Business \& Economics, 10(3), 123-149.

Lobato, J. (2003). How design experiments can inform a rethinking of transfer and vice versa. Educational Researcher, 32(1), 17-20. doi:10.3102/0013189X032001017 
Lobato, J. (2006). Alternative perspectives on the transfer of learning. History, issues, and challenges for future research. The Journal of the Learning Sciences, 15(4), 431-449. doi:10.1207/s15327809jls1504_1

Locke, E. A., \& Latham, G. P. (1990). A theory of goal setting \& task performance. Englewood Cliffs, NJ: Prentice Hall.

Lourenco, D., \& Ferreira, A. I. (2019). Self-regulated learning and training effectiveness. International Journal of Training and Development, 23(2), 117-134. doi:10.1111/ijtd.12149

Lutz, W., \& Grawe, K. (2007). Psychotherapieforschung: Grundlagen, Konzepte und neue Trends. In B. Strauß, F. Hohagen, \& F. Caspar (Hrsg.), Lehrbuch Psychotherapie, Bd. 1 (S. 727-768). Göttingen: Hogrefe.

Madagamage, G. T., Warnakulasooriya, B. N. F., \& Wickramasuriya, H. V. A. (2014). Factors influencing motivation to transfer training: An empirical study of a government sector training program in Sri Lanka. Tropical Agricultural Research, 26(1), 12-25. doi:10.4038/tar.v26i1.8068

Mandl, H., Gruber, H., \& Renkl, A. (2002). Situiertes Lernen in multimedialen Lernumgebungen. In L. J. Issing \& P. Klimsa (Hrsg.), Information und Lernen mit Multimedia und Internet (S. 139-149). Weinheim: Beltz, Psychologie Verlags Union.

Mandl, H., Prenzel, M., \& Gräsel, C. (1992). Das Problem des Lerntransfers in der betrieblichen Weiterbildung. Unterrichtswissenschaft, 20, 126-143.

Martin, R., Epitropaki, O., \& O’Broin, L. (2017). Methodological issues in leadership training research: In pursuit of causality. In R. Galavan, K. Sund, \& G. Hodgkinson (Hrsg.), Methodological Challenges and Advances in Managerial and Organizational Cognition (New Horizons in Managerial and Organizational Cognition) (Vol. 2, S. 73-94). online: Emerald Publishing Limited.

Martins, L. B., Zerbini, T., \& Medina, F. J. (2019). Impact of online training on behavioral transfer and job performance in a large organization. Journal of Work and Organizational Psychology, 35(1), 27-37. doi:10.5093/jwop2019a4

Massenberg, A.-C., \& Kauffeld, S. (2015). Hilf mir (nicht immer): Eine moderierte Mediationsanalyse zum Einfluss der Unterstützung durch die Führungskraft auf Transfermotivation und Lerntransfer. Zeitschrift für Erziehungswissenschaften, 18(1), 145-167. doi:10.1007/s11618-014-0603-5

Massenberg, A.-C., Schulte, E. M., \& Kauffeld, S. (2017). Never too early: Learning transfer system factors affecting motivation to transfer before and after training programs. Human Resource Development Quarterly, 28(1), 55-85. doi:10.1002/hrdq.21256

Massenberg, A.-C., Spurk, D., \& Kauffeld, S. (2015). Social support at the workplace, motivation to transfer and training transfer: A multilevel indirect effects model. International Journal of Training and Development, 19(3), 161-178. doi:10.1111/ijtd.12054

Medina, M. N. (2017). Training motivation and satisfaction: The role of goal orientation and offshoring perception. Personality and Individual Differences, 105, 287-293. doi:10.1016/j.paid.2016.10.016

Mensmann, M., \& Frese, M. (2019). Who stays proactive after entrepreneurship training? Need for cognition, personal initiative maintenance, and well-being. Journal of Organizational Behavior, 40(1), 20-37. doi:10.1002/job.2333

Miiro, R. F., Matsiko, F. B., \& Mazur, R. E. (2014). Training and farmers' organizations' performance. Journal of Agricultural Education and Extension, 20(1), 65-78. doi:10.1080/1389224X.2013.803987

Miles, J. N. V., \& Shevlin, M. (2001). Applying regression and correlation: A guide for students and researchers. London: Sage.

Moosbrugger, H., \& Rauch, W. (2009). Das Allgemeine Lineare Modell in der Evaluationsforschung. In H. Holling (Hrsg.), Grundlagen und statistische Methoden der Evaluationsforschung (S. 285-334). Göttingen: Hogrefe. 
Morgeson, F. P., DeRue, S., \& Karam, E. P. (2010). Leadership in teams: A functional approach to understanding leadership structures and processes. Journal of Management, 36(1), 5-39. doi:10.1177/0149206309347376

Morris, S. B., \& DeShon, R. P. (2002). Combining effect size estimates in meta-analysis with repeated measures and independent-groups designs. Psychological Methods, 7(1), 105-125. doi:10.1037/1082-989X.7.1.105

Muduli, A., \& Raval, D. (2018). Examining the role of work context, transfer design and transfer motivation on training transfer. European Journal of Training and Development, 42(3/4), 266-282. doi:10.1108/EJTD-09-2017-0078

Nafukho, F. M., Alfred, M., Chakraborty, M., Johnson, M., \& Cherrstrom, C. A. (2017). Predicting workplace transfer of learning: A study of adult learners enrolled in a continuing professional education training program. European Journal of Training and Development, 41(4), 327-353. doi:10.1108/EJTD-10-2016-0079

$\mathrm{Ng}, \mathrm{K} . \mathrm{H}$. (2015). Supervisory practices and training transfer: Lessons from Malaysia. Asia Pacific Journal of Human Resources, 53(2), 221-240. doi:10.1111/1744-7941.12044

Ng, K. H., \& Ahmad, R. (2018). Personality traits, social support, and training transfer: The mediating mechanism of motivation to improve work through learning. Personnel Review, 47(1), 39-59. doi:10.1108/PR-08-2016-0210

Nik Nazli, N. N. N., \& Sheikh Khairudin, S. M. H. (2018). The factors that influence transfer of training and its effect on organizational citizenship behaviour. Journal of Workplace Learning, 30(2), 121-146. doi:10.1108/JWL-09-2017-0080

Noe, R. A., \& Schmitt, N. (1986). The influence of trainee attitudes on training effectiveness: Test of a model. Personnel Psychology, 39(3), 497-523. doi:10.1111/j.17446570.1986.tb00950.x

Noe, R. A., \& Wilk, S. L. (1993). Investigation of the factors that influence employees' participation in development activities. Journal of Applied Psychology, 78(2), 291-302. doi:10.1037/0021-9010.78.2.291

North, K., Reinhardt, K., \& Sieber-Suter, B. (2018). Kompetenzmanagement in der Praxis: Mitarbeiterkompetenzen systematisch identifizieren, nutzen und entwickeln. Mit vielen Praxisbeispielen (3. Aufl.). Wiesbaden: Springer.

Nunnally, J. C., \& Bernstein, I. H. (1994). Psychometric theory (3. Aufl.). New York, NY: McGraw-Hill.

O'Leonard, K. (2014). The corporate learning factbook: Benchmarks, trends and analysis of the U.S. training market (B. Associates Ed.). Oakland, CA.

Park, S., Kang, H.-S., \& Kim, E.-J. (2018). The role of supervisor support on employees' training and job performance: An empirical study. European Journal of Training and Development, 42(1/2), 57-74. doi:10.1108/EJTD-06-2017-0054

Park, Y., Lim, D. H., \& Chang, J. (2017). Trainee versus supervisor assessment of training transfer: Mediational analysis of transfer variables. Asia Pacific Journal of Human Resources, 55(2), 192-212. doi:10.1111/1744-7941.12116

Patton, M. Q. (1997). Utilization-focused evaluation: The new century text (3. Aufl.). Thousand Oaks, CA: Sage.

Paulsen, H. F. K., \& Kauffeld, S. (2017). Linking positive affect and motivation to transfer within training: A multilevel study. International Journal of Training and Development, 21(1), 35-52. doi:10.1111/ijtd.12090

Perez-Soltero, A., Aguilar-Bernal, C., Barcelo-Valenzuela, M., Sanchez-Schmitz, G., MeroñoCerdan, A. L., \& Fornes-Rivera, R. D. (2019). Knowledge transfer in training processes: Towards an integrative evaluation model. IUP Journal of Knowledge Management, 17(1), 7-40.

Peters, S., Cossette, M., Bates, R., Holton, E., Hansez, I., \& Faulx, D. (2014). The influence of transfer climate and job attitudes on the transfer process: Modeling the direct and indirect effects. Journal of Personnel Psychology, 13(4), 157-166. doi:10.1027/18665888/a000109 
Pfeiffer, J. W., Heslin, R., \& Jones, J. E. (1976). Instrumentation in human relations training: A guide to 92 instruments with wide application to the behavioral sciences. San Diego: University Associates.

Phillips, J. J., \& Schirmer, F. C. (2008). Return on Investment in der Personalentwicklung. Der 5-Stufen-Evaluationsprozess (2. Aufl.). Berlin [u.a.]: Springer.

Piezzi, D. (2002). Transferförderung in der betrieblichen Weiterbildung - Die Bedeutung der Arbeitsumgebung und der Integration der Weiterbildung in die Unternehmensführung. Paderborn: Eusl-Verlag.

Podsakoff, P. M., MacKenzie, S. B., Lee, J.-Y., \& Podsakoff, N. P. (2003). Common method biases in behavioral research: A critical review of the literature and recommended remedies. Journal of Applied Psychology, 88(5), 879-903. doi:10.1037/00219010.88.5.879

Podsakoff, P. M., MacKenzie, S. B., \& Podsakoff, N. P. (2012). Sources of method bias in social science research and recommendations on how to control it. Annual Review of Psychology, 63(1), 539-569. doi:10.1146/annurev-psych-120710-100452

Poell, R. F. (2017). Time to 'flip' the training transfer tradition: Employees create learning paths strategically. Human Resource Development Quarterly, 28(1), 9-15. doi:10.1002/hrdq. 21279

Powell, K. S., \& Yalcin, S. (2010). Managerial training effectiveness: A meta-analysis 19522002. Personnel Review, 39(2), 227-241. doi:10.1108/00483481011017435

Quesada-Pallarès, C., \& Gegenfurtner, A. (2015). Toward a unified model of motivation for training transfer: a phase perspective. Zeitschrift für Erziehungswissenschaften, 18, 107-121. doi:10.1007/s11618-014-0604-4

Rahyuda, A. G., Soltani, E., \& Syed, J. (2018). Preventing a relapse or setting goals? Elucidating the impact of post-training transfer interventions on training transfer performance. International Journal of Training Research, 16(1), 61-82. doi:10.1080/14480220.2017.1411287

Rank, B., \& Wakenhut, R. (1998). Sicherung des Praxistransfers im Führungskräftetraining. München [u.a.]: Hampp.

Reid, D. H., Rotholz, D. A., Parsons, M. B., Morris, L., Braswell, B. A., Green, C. W., \& Schell, R. M. (2003). Training human service supervisors in aspects of PBS evaluation of a statewide, performance-based program. Journal of Positive Behavior Interventions, 5(1), 35-46. doi:10.1177/10983007030050010601

Reinhold, S., Gegenfurtner, A., \& Lewalter, D. (2018). Social support and motivation to transfer as predictors of training transfer: Testing full and partial mediation using meta-analytic structural equation modelling. International Journal of Training and Development, 22(1), 1-14. doi:10.1111/ijtd.12115

Renta-Davids, A.-I., Jiménez-González, J.-M., Fandos-Garrido, M., \& González-Soto, A.-P. (2014). Transfer of learning: Motivation, training design and learning-conducive work effects. European Journal of Training and Development, 38(8), 1-19. doi:10.1108/EJTD03-2014-0026

Roberts, Z., Rogers, A., Thomas, C. L., \& Spitzmueller, C. (2018). Effects of proactive personality and conscientiousness on training motivation. International Journal of Training and Development, 22(2), 126-143. doi:10.1111/ijtd.12122

Rogers, C. R. (1973). Entwicklung der Persönlichkeit: Psychotherapie aus der Sicht eines Therapeuten. Stuttgart: Klett.

Rosenberg, M. (1965). Society and the adolescent self-image (2. Aufl.). Princeton, NJ: Princeton University Press.

Rosenberg, M. (1989). Society and the adolescent self-image (3. Aufl.). Middeltown, CT: Wesleyan University Press.

Rosenstiel, L. v. (2006). Führung. In H. Schuler (Hrsg.), Lehrbuch der Personalpsychologie (2. Aufl., S. 353-384). Göttingen [u.a.]: Hogrefe. 
Rosenstiel, L. v. (2012). Wo die Grenzen nachhaltiger Weiterbildung liegen und was mehr Erfolg verspricht. Personal Quarterly, 64(1), 6-9.

Rosenstiel, L. V., Molt, W., Rüttinger, B., \& Wissner, B. (2005). Organisationspsychologie (9. Aufl.). Stuttgart: Kohlhammer.

Rosenstiel, L. v., \& Nerdinger, F. W. (2011). Grundlagen der Organisationspsychologie Basiswissen und Anwendungshinweise (7. Aufl.). Stuttgart: Schäffer-Poeschel.

Roth, H. (1971). Pädagogische Anthropologie (Bd. 2). Hannover: Schrödel.

Rouiller, J. Z., \& Goldstein, I. L. (1993). The relationship between organizational transfer climate and positive transfer of training. Human Resource Development Quarterly, 4(4), 377390. doi:10.1002/hrdq.3920040408

Rubin, D. B. (1976). Inference and missing data. Biometrika, 63(3), 581-592. doi:10.1093/biomet/63.3.581

Rubin, D. B. (1987). Multiple Imputation for nonresponse in surveys. New York, NY: John Wiley \& Sons.

Rubin, D. B. (1996). Multiple Imputation after 18+ years. Journal of the American Statistical Association, 91(434), 473-489.

Russ-Eft, D. F., Dickison, P., \& Levine, R. (2010). Taking the pulse of training transfer: Instructor quality and EMT certification examination results. Human Resource Development Quarterly, 21(3), 291-306. doi:10.1002/hrdq.20052

Sahoo, M., \& Mishra, S. (2017). Training evaluation and motivation to transfer training: A review of literature. Parikalpana: KIIT Journal of Management, 13(2), 17-28. doi:10.23862/kiitparikalpana/2017/v13/i2/164517

Sahoo, M., \& Mishra, S. (2019). Effects of trainee characteristics, training attitudes and training need analysis on motivation to transfer training. Management Research Review, 42(2), 215-238. doi:10.1108/MRR-02-2018-0089

Saks, A. M., \& Burke, L. A. (2012). An investigation into the relationship between training evaluation and the transfer of training. International Journal of Training and Development, 16(2), 118-127. doi:10.1111/j.1468-2419.2011.00397.x

Salas, E., \& Kosarzycki, M. P. (2003). Why don't organizations pay attention to (and use) findings from the science of training? Human Resource Development Quarterly, 14(4), 487-491. doi:10.1002/hrdq.1081

Salas, E., Tannenbaum, S. I., Kraiger, K., \& Smith-Jentsch, K. A. (2012). The science of training and development in organizations: What matters in practice. Psychological Science in the Public Interest, 13(2), 74-101. doi:10.1177/1529100612436661

Schaper, N. (2011). Aus- und Weiterbildung: Konzepte der Trainingsforschung. In F. W. Nerdinger, G. Blickle, \& N. Schaper (Hrsg.), Arbeits- und Organisationspsychologie mit 34 Tabellen (2. Aufl., S. 425-450). Berlin [u.a.]: Springer.

Schindler, L. A., \& Burkholder, G. J. (2016). A mixed methods examination of the influence of dimensions of support on training transfer. Journal of Mixed Methods Research, 10(3), 292-310. doi:10.1177/1558689814557132

Schwartz, D. L., Bransford, J. D., \& Sears, D. (2005). Efficiency and innovation in transfer. In P. J. Mestre (Hrsg.), Transfer of Learning from a Moderns Multidisciplinary Perspective (S. 1-51). New York, NY: Information Age Publishing.

Schwartz, J., Bersin, J., \& Pelster, B. (2014). Human Capital Trends 2014 Survey. Verfügbar unter https://www2.deloitte.com/insights/us/en/focus/human-capital-trends/2014/humancapital-trends-2014-survey-top-10-findings.html (22.1.2018).

Schwarzer, R., \& Jerusalem, M. (1999). Skalen zur Erfassung von Lehrer- und Schülermerkmalen. Dokumentation der psychometrischen Verfahren im Rahmen der wissenschaftlichen Begleitung des Modellversuchs Selbstwirksame Schulen. Berlin: Freie Universität Berlin. 
Schyns, B. (2002). Überprüfung einer deutschsprachige Skala zum Leader-Member-ExchangeAnsatz. Zeitschrift für Differentielle und Diagnostische Psychologie, 23(2), 235-245. doi:10.1024//0170-1789.23.2.235

Schyns, B., \& Collani, G. v. (2002). A new occupational self-efficacy scale and its relation to personality constructs and organizational variables. European Journal of Work and Organizational Psychology, 11(2), 219-241. doi:10.1080/13594320244000148

Scriven, M. (1991). Evaluation Thesaurus (4. Aufl.). Newbury Park, CA: Sage.

Seeg, B. (2020). Nachhaltige Personalentwicklung. In A. Schütz, C. Köppe, \& M. Andresen (Hrsg.), Was Führungskräfte über Psychologie wissen sollten: Theorie und Praxis für den Umgang mit Mitarbeitenden. Bern: Hogrefe.

Seeg, B., \& Schütz, A. (2019). Trainingstransfer. In M. A. Wirtz (Hrsg.), Dorsch - Lexikon der Psychologie. Göttingen: Hogrefe.

Seiberling, C., \& Kauffeld, S. (2017). Volition to transfer: Mastering obstacles in training transfer. Personnel Review, 46(4), 809-823. doi:10.1108/PR-08-2015-0202

Seidel, J. (2012). Transferkompetenz und Transfer: Theoretische und empirische Untersuchung zu den Wirksamkeitsbedingungen betrieblicher Weiterbildung. Landau: Verlag Empirische Pädagogik.

Seyda, S., \& Placke, B. (2017). IW-Weiterbildungserhebung 2017 - Kosten und Nutzen betrieblicher Weiterbildung. Verfügbar unter https://www.iwkoeln.de/studien/iwtrends/beitrag/susanne-seyda-beate-placke-die-neunte-iw-weiterbildungserhebung369145.html (22.01.2018).

Shen, J., \& Tang, C. (2018). How does training improve customer service quality? The roles of transfer of training and job satisfaction. European Management Journal, 36(6), 708-716. doi:10.1016/j.emj.2018.02.002

Shewhart, W. A. (1939). Statistical method from the viewpoint of quality control. Lancaster, PA: Lancaster Press.

Sitzmann, T., \& Weinhardt, J. M. (2018). Training engagement theory: A multilevel perspective on the effectiveness of work-related training. Journal of Management, 44(2), 732-756. doi:10.1177/0149206315574596

Smy, V., Shelton, K., Tombs, M., \& Patrick, J. (2016). Perceived transformational leadership, instructor behaviors, and motivation to learn: A mediated model. Le Travail Humain: $A$ Bilingual and Multi-Disciplinary Journal in Human Factors, 79(2), 169-185. doi:10.3917/th.792.0169

Solga, M. (2011a). Evaluation der Personalentwicklung. In J. Ryschka, M. Solga, \& A. Mattenklott (Hrsg.), Praxishandbuch Personalentwicklung: Instrumente, Konzepte, Beispiele (3. Aufl., S. 369-399). Wiesbaden: Gabler.

Solga, M. (2011b). Förderung von Lerntransfer. In J. Ryschka, M. Solga, \& A. Mattenklott (Hrsg.), Praxishandbuch Personalentwicklung: Instrumente, Konzepte, Beispiele (3. Aufl., S. 339-368). Wiesbaden: Gabler.

Solga, M., Ryschka, J., \& Mattenklott, A. (2011). Personalentwicklung: Gegenstand, Prozessmodell, Erfolgsfaktoren. In J. Ryschka, M. Solga, \& A. Mattenklott (Hrsg.), Praxishandbuch Personalentwicklung: Instrumente, Konzepte, Beispiele (3. Aufl., S. 1934). Wiesbaden: Gabler.

Solomon, R. L. (1949). An extension of control group design. Psychological Bulletin, 46(2), $137-$ 150. doi:10.1037/h0062958

Sonntag, K., Schaper, N., \& Friebe, J. (2005). Erfassung und Bewertung von Merkmalen untemehmensbezogener Lernkulturen. In ABWF/QEM (Hrsg.), Kompetenzmessung im Unternehmen (S. 19-339). Münster: Waxmann.

Sørensen, P. (2017). What research on learning transfer can teach about improving the impact of leadership-development initiatives. Consulting Psychology Journal: Practice and Research, 69(1), 47-62. doi:10.1037/cpb0000072 
Sparr, J. L., Knipfer, K., \& Willems, F. (2017). How leaders can get the most out of formal training: The significance of feedback-seeking and reflection as informal learning behaviors. Human Resource Development Quarterly, 28(1), 29-54. doi:10.1002/hrdq.21263

Spector, P. E. (2006). Method variance in organizational research: truth or urban legend? Organizational Research Methods, 9(2), 221-232.

Staudt, E., \& Kriegesmann, B. (1999). Weiterbildung: Ein Mythos zerbricht. Der Widerspruch zwischen überzogenen Erwartungen und Mißerfolgen der Weiterbildung (Vol. 178). Bochum: IAI.

Stockmann, R. (2000). Evaluationsforschung. Opladen: Leske und Budrich.

Stufflebeam, D. L. (1983). The CIPP model for program evaluation. In G. F. Madaus, M. Scriven, \& D. L. Stufflebeam (Hrsg.), Evaluation models: Viewpoints on educational and human services evaluation (S. 117-141). Boston, MA: Kluwer.

Stufflebeam, D. L. (2004). The 21st century CIPP model: Origins, development, and use. In M. C. Alkin (Hrsg.), Evaluation Roots: Tracins theorists' views ans influences (S. 245-266). Thousand Oaks, CA: Sage.

Stufflebeam, D. L. (2014). Evaluation Theory, Models, and Applications (2. Aufl.). Somerset: Wiley.

Stufflebeam, D. L., \& Zhang, G. (2017). The CIPP evaluation model: How to evaluate for improvement and accountability. New York, NY: Guilford.

Sua Wui, C., Mohd Haizam Mohd, S., \& Chong Aik, L. (2018). The effects of mobile learning factors and training transfer on the effective organisational learning in Malaysian Oil and Gas Industry. Asian Journal of Innovation \& Policy, 7(2), 310-337. doi:10.7545/ajip.2018.7.2.310

Suchman, E. A. (1967). Evaluative Research: Principles and Practice. New York, NY: Russell Sage Foundation.

Tabachnick, B. G., \& Fidell, L. S. (2014). Using multivariate statistics (6. Aufl.). Harlow, UK: Pearson.

Taylor, P. J., Russ-Eft, D. F., \& Chan, D. W. L. (2005). A meta-analytic review of Behavior Modeling Training. Journal of Applied Psychology, 90(4), 692-709. doi:10.1037/00219010.90.4.692

Taylor, P. J., Russ-Eft, D. F., \& Taylor, H. (2009). Transfer of management training from alternative perspectives. Journal of Applied Psychology, 94(1), 104-121. doi:10.1037/a0013006

Tett, R. P., \& Burnett, D. D. (2003). A personality trait-based interactionist model of job performance. Journal of Applied Psychology, 88(3), 500-517. doi:10.1037/00219010.88.3.500

Tharenou, P., Sakes, A., \& Moore, C. (2007). A review and critique of research on training and organizational-level outcomes. Human Resource Management Review, 17(3), 251-273. doi:10.1016/j.hrmr.2007.07.004

Thorndike, E. L., \& Woodworth, R. S. (1901). The influence of improvement in one mental function upon the efficiency of other functions. Psychological Review, 8(3), 247-261.

Tonhäuser, C., \& Bücker, L. (2016). Determinants of transfer of training: A comprehensive literature review. International Journal for Research in Vocational Education and Training, 3(2), 127-165. doi:10.13152

Torp, S. (2008). How a health and safety management training program may improve the working environment in small- and medium-sized companies. Journal of Occupational and Environmental Medicine, 50(3), 263-271. doi:10.1097/JOM.0b013e318163866f

Towler, A., Watson, A., \& Surface, E. A. (2014). Signaling the importance of training. Journal of Managerial Psychology, 29(7), 829-849. doi:10.1108/JMP-03-2012-0092

Urban, D., \& Mayerl, J. (2018). Angewandte Regressionsanalyse: Theorie, Technik und Praxis (Vol. 5). Wiesbaden: Springer VS. 
Vignoli, M., \& Depolo, M. (2019). Transfer of training process. When proactive personality matters? A three-wave investigation of proactive personality as a trigger of the transfer of training process. Personality and Individual Differences, 141, 62-67. doi:10.1016/j.paid.2018.12.027

Vignoli, M., Mariani, M. G., Guglielmi, D., \& Violante, F. S. (2018). Leadership styles and selfefficacy in determining transfer intentions of safety training. Journal of Workplace Learning, 30(1), 65-76. doi:10.1108/JWL-01-2017-0001

Volmer, J. (2015). Followers' daily reactions to social conflicts with supervisors: The moderating role of core self-evaluations and procedural justice perceptions. The Leadership Quarterly, 26(5), 719-731. doi:10.1016/j.leaqua.2015.01.005

Wang, G. G., \& Wilcox, D. (2014). Training evaluation: Knowing more than is practiced. Advances in Developing Human Resources, 8(4), 528-539. doi:10.1177/1523422306293007

Weinbauer-Heidel, I. (2016). Transferförderung in der betrieblichen Weiterbildungspraxis: Warum transferfördernde Maßnahmen (nicht) implementiert werden. Wiesbaden: Springer.

Wen, M. L.-Y., \& Lin, D. Y.-C. (2014a). How supportive transfer climate affects individual's motivation to training transfer. International Journal of Learning and Developmen, 4(1), 83-97. doi:10.5296/ijld.v4i1.5074

Wen, M. L.-Y., \& Lin, D. Y.-C. (2014b). Trainees' characteristics in training transfer: The relationship among self-efficacy, motivation to learn, motivation to transfer and training transfer. International Journal of Human Resource Studies, 4(1), 114-129. doi:10.5296/ijhrs.v4i1.5128

Westermann, R., \& Siemer, M. (2009). Aufstellen und Prüfen von Hypothesen. In H. Holling (Hrsg.), Grundlagen und statistische Methoden der Evaluationsforschung (S. 163-206). Göttingen: Hogrefe.

Wexley, K. N., \& Latham, G. P. (1981). Developing and training human resources in organizations. Glenview, IL: Scott Foresman, \& Company.

Will, H., Winteler, A., \& Krapp, A. (1987). Von der Erfolgskontrolle zur Evaluation. In H. Will, A. Winteler, \& A. Krapp (Hrsg.), Evaluation in der beruflichen Aus- und Weiterbildung: Konzepte und Strategien (S. 11-42). Heidelberg: Sauer.

Wittmann, W. W. (1990). Brunswik-Symmetrie und die Konzeption der Fünf-Datenboxen. Ein Rahmenkonzept für umfassende Evaluationsforschung. Zeitschrift für Pädagogische Psychologie, 4, 241-251.

Wittmann, W. W. (2009). Evaluationsmodelle. In H. Holling (Hrsg.), Grundlagen und statistische Methoden der Evaluationsforschung (S. 59-98). Göttingen: Hogrefe.

Yaghi, A., Goodman, D., Holton, E. F., \& Bates, R. A. (2008). Validation of the Learning Transfer System Inventory: A study of supervisors in the public sector in Jordan. Human Resource Development Quarterly, 19(3), 241-262. doi:10.1002/hrdq.1238

Yip Foon, Y. (2019). The moderating effect of transfer climate on the joint relationship between trainees' personality and transfer training. Journal of Management and Marketing Review, 4(1), 79-85.

Zamani, N., Ataei, P., \& Bates, R. A. (2016). The use of the Persian translation of the Learning Transfer System Inventory in the context of agricultural sustainability learning in Iran. International Journal of Training and Development, 20(1), 92-104. doi:10.1111/ijtd.12071

Zhang, J. (1999). Effects of management training on trainees' learning, job performance, and organization results: A meta-analysis of evaluation studies from 1983-1997. Unveröffentlichte Dissertation. Oklahoma State University.

Zohar, D. (2002). Modifying supervisory practices to improve subunit safety: A leadership-based intervention model. Journal of Applied Psychology, 87(1), 156-163. doi:10.1037/00219010.87.1.156 
Zollondz, H.-D. (2014). Lexikon Qualitätsmanagement: Handbuch des Modernen Managements auf der Basis des Qualitätsmanagements - Edition Versicherungsmanagement. Berlin [u.a]: Walter de Gruyter $\mathrm{GmbH}$.

Zumrah, A. R. (2015). Examining the relationship between perceived organizational support, transfer of training and service quality in the Malaysian public sector. European Journal of Training and Development, 39(2), 143-160. doi:10.1108/EJTD-09-2014-0066

Zumrah, A. R., \& Boyle, S. (2015). The effects of perceived organizational support and job satisfaction on transfer of training. Personnel Review, 44(2), 236-254. doi:10.1108/PR02-2013-0029 